\title{
TRANSMISSION SECONDARY EMISSION FROM THIN FILMS OF ALKALI HALIDES
}

by

Jorge Llacer

\author{
Technical Report \\ Prepared Under \\ Contract AT(04-3)-515 \\ for the USAEC
}

San Francisco Operations Office

Printed in USA. Available from CFSTI, National Bureau of Standards, U.S. Department of Commerce, Springfield, Virginia 22151 Price: Printed Copy $\$ 3.00 ;$ Microfiche $\$ 0.65$. 


\section{ACKNOWLEDGEMENTS}

The author is very grateful to Dr. Edward L. Garwin for very sustained moral and physical support and valuable advice during the course of the work reported here, to Professor John L. Moll for his encouragement and advice during the period of gestation of the electron-phonon interaction, to John C. Edgecumbe for lending me some of his experience (which made the measurements more feasible), and to Professor W. E. Spicer for some very timely advice regarding the subject of this investigation. The assistance of the Technical Staff of the Physical Electronics Group and of the Machine Shop of the Central Laboratory, SLAC, has been very good at all times and I am grateful for it. I would also like to thank Steve Long for his help with some preliminary measurements. 
Introduction . . . . . . . . . . . . . . . $\frac{\text { Page }}{1}$

I. Theory ...................... 3

A. Preliminary Considerations ............. 3

B. Relationship Between the Energy Loss Suffered by Primary

Electrons and the Generation of Secondaries . . . . . . 5

C. The Loss of Energy by Electrons Below $12 \mathrm{keV}$. . . . . . . 5

1. Comments on published theoretical results . . . . . 5

2. The definition of range and Bethe's equation . . . . 10

3. Finding the rate of energy loss from experimental results 15

4. Application to composite films . . . . . . . . 22

5. The secondary electron generation function . . . . . 23

D. The Escape Mechanism of Secondary Electrons . . . . . 27

1. Preliminary considerations . . . . . . . . . 27

2. The electron-phonon interaction . . . . . . . . 28

3. Rate and angle of scattering . . . . . . . . . 39

4. The Boltzmann transport equation . . . . . . . 50

5. Monte Carlo solution . . . . . . . . . . 53

6. Results of computations . . . . . . . . . 62

7. Dependence on effective mass and temperature . . . . 80

II. Experiments . . . . . . . . . . . . . . 82

A. Preliminary Considerations . . . . . . . . . 82

B. Measurement of Secondary Yield in Uncharged Films . . . 84

1. Description of equipment . . . . . . . . . . . 84

a. Tube assembly . . . . . . . . . . 84

b. Electronic circuits . . . . . . . . . 88 
2. Testing of equipment ............ 93

a. Evaluation of the tube performance and systematic error ................. 93

b. Calibration of electronic system and evaluation of random errors ........... . . 99

3. Preliminary measurements .......... 103

a. Yield of substrates ........... 103

b. Effect of pressure and evaporation rate on the yield of alkali halides .......... 103

4. Measurements on alkali halides ........ 105

C. Measurement of Energy Distribution in Uncharged Films . . 114

1. Principles of operation . . . . . . . . . 115

a. Kelvin probe .............. 115

b. Retarding potential measurements with plane geometry .............. 121

2. Description of equipment . . . . . . . . 129

a. The Kelvin probe tube . . . . . . . . 129

b. The electronic system . . . . . . . . 132

(1) Regulated primary beam power supply . . . 132

(2) Dynode circuit . . . . . . . . 136

(3) Kelvin probe circuit . . . . . . 136

3. Calibration and initial measurements . . . . . 138

4. Measurements on alkali halide films . . . . . . 141 
III. Quantitative Analysis of Results . . . . . . . . . 150

A. The Model for Transmission Secondary Emission . . . . 150

1. Mathematical model ........... 150

2. Calculations for the selected alkali halides ..... 153

a. Cesium Iodide . . . . . . . . . . 153

b. Potassium Chloride . . . . . . . . 156

c. Sodium and Lithium Fluoride . . . . . . . 159

3. Temperature Dependence . . . . . . . . 160

B. Final Discussion and Conclusion . . . . . . . . . 161

References ..................... 167 


\section{LIST OF TABLES}

1. Comparison between energy loss computed from Bethe's equation

[Eq. (2)] and from the experimental results of Kanter, ${ }^{6}$ and

Kanter and Sternglass. ${ }^{9}$. . . . . . . . . . . 13

2. Sample computation of energy loss . . . . . . . . 16

3. Sample computation of backscattering correction to energy loss . . 19

4. Substrate transmission data for calculation of energy loss in composite films . . . . . . . . . . . . . 23

5. Data on polar insulators, from Bak. ${ }^{52}$. . . . . . . . 45

6. Data on position of vacuum level and conduction band edge from the literature................... 59

7. Values of $\mathrm{P}_{0}\left(\mathcal{E}_{0}\right)$ and $\mathrm{L}_{\mathrm{S}}$ for $\mathrm{CsI}, \mathrm{KCl}, \mathrm{NaF}$, and $\mathrm{LiF}$ as a function of initial energy $\xi_{0}$. . . . . . . . . . . 70

8. Correlation data for electrons in a $250 \AA$ CsI film . . . . . . 76

9. Measured $I_{G_{0}}$ during the evaluation of tube performance . . . 94

10. Comparison of yield measurements with and without the Faraday cage at the collector $\mathrm{C}$. . . . . . . . . . . 


\section{LIST OF ILLUSTRATIONS}

1. Average

1. Average electron energy vs. depth of penetration in a $1200 \mathrm{~A}$ film of aluminum. Results of integrating Bethe's equation [Eq. (2)] • • 12

2. Energy loss and its derivative for an electron beam of $E_{p}=6 \mathrm{keV}$ in aluminum . . . . . . . . . . . 17

3. Energy loss per unit thickness in a $100 \mu \mathrm{g} / \mathrm{cm}^{2}$ film of $\mathrm{Al}$, with and without the linear loss correction . . . . . . . 21

4. Energy loss per unit length for a CsI composite film . . . . . . 24

5. Energy loss per unit length for a $\mathrm{KCl}$ composite film . . . . . 25

6. Relationship between vectors $\vec{q}, \vec{k}$, and $\vec{k}$ in wave-vector space

(a) For phonon annihilation interaction .......... 40

(b) For phonon creation interaction . . . . . . . . . 40 40

7. $\mathrm{W}_{\overrightarrow{\mathrm{k}}}^{-}$and $\mathrm{W}_{\overrightarrow{\mathrm{k}}}^{+}$, the total scattering rates as a function of electron energy, for $\mathrm{CsI}$ and $\mathrm{KCl}$. . . . . . . . . . . 44

8. Mean free path vs. electron energy for several alkali halides . . 47

9. Scattering angular probability distribution for CsI, phonon annihilation ............... 51

10. Coordinates for the computation of new momentum coordinates upon scattering . . . . . . . . . . . . . 57

11. Film coordinates for computations of escape probabilities . . . . 63

12. Probabilities of escape for electrons generated at a position $x$ in a $250 \AA$ film of CsI, with initial energy $\varepsilon_{0}$ and initial angle $\theta \quad . \quad 65,66$

13. Probabilities of escape for electrons generated as a position $\mathrm{x}$ in a $250 \AA$ film of CsI, with initial energy $\mathcal{E}_{0}$, and an isotropic angular distribution . . . . . . . . . . . . 68 
14. Showing the near exponential character of the escape probability

(a) $\mathrm{P}\left(\mathcal{G}_{0}=.25 \mathrm{eV}, \mathrm{x}\right)$ for $250 \AA$ film of CsI ......... 69

(b) $\mathrm{P}\left(\mathcal{E}_{0}=.5 \mathrm{eV}, \mathrm{x}\right)$ for $250 \AA$ film of CsI ........ 69

15. Exit energy distribution for electrons generated in the forward direction with $\mathscr{E}_{0}=1 \mathrm{eV}$ in a $250 \AA$ film of CsI . . . . . . . . 72

16. Probabilities of escape for electrons generated isotropically with $\mathcal{E}_{0}=.5 \mathrm{eV}$ at $\mathrm{x}=25 \AA$ in a $250 \AA$ film of $\mathrm{CsI}$. . . . . . . .

17. Mean energy loss (a) and standard deviation (b) for escaping electrons generated isotropically at position $x$ with energy $\mathcal{G}_{0}$ in a $250 \AA$ CsI film . . . . . . . . . . . . . . . . . . . .

18. Mean energy loss (a) and standard deviation (b) for escaping electrons generated isotropically at position $\mathrm{x}$ with energy $\mathscr{\delta}_{0}$ in a $250 \AA \mathrm{A} \mathrm{KCl} \mathrm{film}$. . . . . . . . . . . . . . . . .

19. Mean energy loss (a) and standard deviation (b) for escaping electrons generated isotropically at position $\mathrm{x}$ with energy $\mathfrak{\xi}_{0}$ in a $250 \AA \mathrm{NaF}$ film . . . . . . . . . . . . . . . . .

20. Mean energy loss (a) and standard deviation (b) for escaping electrons generated isotropically at position x with energy $\mathcal{E}_{0}$ in a $250 \stackrel{\circ}{\mathrm{A}} \mathrm{LiF}$ film . . . . . . . . . . . . . . . . . . .

21. Schematic of the tube used for measurement of secondary yield . . . 85

22. Schematic of circuits used for measurement of secondary yield . . . 89

23. Retarding potential measurements of $\mathrm{G}_{0}-\mathrm{Ta}$ foil system . . . . 96

24. Typical oscilloscope pattern in the measurement of pulsed $\delta_{t}$. Vertical scale: $2 \times 10^{-14}$ coulomb $/ \mathrm{cm}$ at peak, horizontal: $20 \mu \mathrm{sec} / \mathrm{cm}$. 102

25. Secondary yield $\delta$, (a), and transmission coefficients $\eta_{t}$, (b), for $1000 \AA \mathrm{Al}_{2} \mathrm{O}_{3}+500 \AA \mathrm{A}$ substrates . . . . . . . . . 
26. Experimental and theoretical secondary yield vs. primary energy for 6 different thicknesses of CsI films . . . . . . . . 107

27. Experimental and theoretical secondary yield vs. primary energy for 6 different thicknesses of $\mathrm{KCl}$ films . . . . . . . . . 108

28. Experimental and theoretical secondary yield vs. primary energy for 6 different thicknesses of NaF films. . . . . . . . . 109

29. Experimental and theoretical secondary yield vs. primary energy for 6 different thicknesses of Li F films. . . . . . . . . . 110

30. Transmission coefficient for standard substrate + CsI films . . . 113

31. Simplified schematic of a Kelvin probe . . . . . . . . . . 116

32. Typical I vs. V characteristic for a high gain dynode . . . . . . . 122

33. Results of computing energy distributions for planar geometry . • . 126

(a) Showing the spread in computed distribution points; from cosine distribution . . . . . . . . . . . . 126

(b) For substantial deviations from cosine distributions . . . . . 127

(c) For extreme deviations from cosine distributions . . . . . 128

34. Schematic drawing of the Kelvin probe tube . . . . . . . . . 130

35. Schematic diagram of regulated primary beam power supply . . . . 133,134

36. Schematic diagram of dynode and Kelvin probe circuits . . . . . . 137

37. Sample measurements of contact potential difference . . . . . . . 142

(a) $\mathrm{Au}-\mathrm{Au}$.................... . . 142

(b) $\mathrm{Au}-\mathrm{Al}$...................... . . 142

(c) $\mathrm{Au}-\mathrm{Al}$, with electron beam on ............ . 142

38. Energy distribution of emitted secondary electrons from CsI films. . 145

39. Energy distribution of emitted secondary electrons from $\mathrm{KCl}$ films. . 146 
40. Energy distribution of emitted secondary electrons from NaF films . . 147

41. Energy distribution of emitted secondary electrons from LiF films . . 148

42. Internal generation function $\mathrm{G}\left(\mathscr{G}_{0}\right)$ for the four selected alkali halides . . . . . . . . . . . . . . . 155

43. Composite escape function $P(x)$ for the four selected alkali halides . . . . . . . . . . . . . . 157 


\section{GLOSSARY OF SOME TERMS PARTICULAR TO THIS REPORT}

$\underline{A}$

$a_{\vec{q}}^{*}:$ creation operator for a phonon with wavevector $\vec{q}$

$a_{\vec{q}}$ : annihilation operator for a phonon with wavevector $\vec{q}$

$\alpha \quad$ : coupling coefficient between an electron and the lattice of an ionic crystal.

$\underline{\mathrm{B}}$

$\beta \quad$ : Parameter dividing $\mathrm{L}_{\mathrm{S}}$ for the temperature or effective mass dependence of escape probability

$\underline{\mathrm{C}}$

C : collector

cpd : contact potential difference

$\underline{D}$

D : dynode

DT : drift tube

$\delta:$ : secondary emission coefficient, yield, gain

$\delta_{\mathrm{t}}:$ transmission gain, equal to $\left(\delta+\eta_{\mathrm{t}}\right)$

$\delta_{\mathrm{D}}:$ dynode yield, as opposed to $\delta_{\mathrm{C}}$

$\delta_{\mathrm{C}}$ : collector yield for primaries transmitted through the dynode

$\underline{\mathrm{E}}$

E : Energy of a primary electron as a function of position in a solid

$E_{p}$ : Initial energy of a primary electron

$\mathrm{E}_{\mathrm{a}}$ : Average energy of electrons transmitted through a film

$E_{c}:$ Critical energy, the energy of an electron with range equal

to the thickness of the film under consideration

$\mathrm{E}_{\mathrm{abs}}:$ average energy of backscattered electrons 
$\xi$ : Energy of a secondary electron inside a film

$\mathcal{E}_{0}:$ initial energy of a secondary electron

$E_{i}$ or $E_{j}$ : exit energy of a secondary electron

e : electronic charge

$\epsilon \quad:$ static dielectric constant

$\epsilon_{\infty}:$ square of the refractive index in the visible range

$\eta$ : fraction of backscattered electrons, backscattering coefficient

$\eta_{\mathrm{t}}$ : fraction of transmitted electrons, transmission coefficient

$\underline{G}$

$\mathrm{G}_{0}$ : grid near entrance face of dynode to suppress emission from that side of it

$G_{1}$ : grid near exit face of dynode to suppress secondary emission from collector and/or dynode

$\gamma: \mathrm{E}_{\mathrm{p}} / \mathrm{E}_{\mathrm{c}}$, reduced primary energy for a given film thickness

$\underline{I}$

$I_{p}:$ primary current

$\underline{\mathrm{K}}$

$\overrightarrow{\mathrm{k}}$ : wavevector of a secondary electron before collision

$\overrightarrow{\mathrm{k}} \quad$ : wavevector of a secondary electron after collision

$\underline{\mathrm{L}}$

$\mathrm{L}_{\mathrm{S}}\left(\mathcal{C}_{0}\right)$ : characteristic escape length for electrons generated isotropically with energy $\mathscr{G}_{0}$

$\underline{\mathrm{M}}$

$\mathrm{m}$ : mass of a free electron

$\mathrm{m}^{*}$ : mass of an electron in a polar crystal 
$\underline{N}$

$\overrightarrow{\mathrm{n}} \quad$ : reciprocal lattice vector

$\underline{\mathrm{O}}$

$\omega \quad$ : angular frequency of longitudinal optical lattice vibrations

$\underline{P}$

$\mathrm{P}_{0}\left(\mathcal{G}_{0}\right)$ : probability of escape of electrons generated isotropically with energy $\mathscr{G}_{0}$ at the exit surface of a film

$\underline{Q}$

$\vec{q} \quad$ : wavevector of a phonon

$\underline{\mathrm{R}}$

$R$ : range of primary electron (definition on p. 10)

$\underline{\mathrm{S}}$

$S$ : exit surface of a dynode

$\mathrm{SE}$ : secondary emission

$\underline{\mathrm{T}}$

$\mathrm{T}$ : temperature, ${ }^{\mathrm{O}} \mathrm{K}$

$\tau$ : thickness of a film. Also, mean free time between collisions

$\underline{\mathrm{V}}$

$\mathrm{V} \quad$ : volume of a crystal

$\underline{\mathrm{W}}$

$\mathrm{W}_{\overrightarrow{\mathrm{k}}}^{-}$: collision rate for electrons with annihilation of one phonon

$\mathrm{W}_{\overrightarrow{\mathrm{k}}}^{+}$: collision rate for electrons with creation of one phonon 


\begin{abstract}
The phenomenon of secondary emission from alkali halides has been studied theoretically and experimentally in substantial detail. Transmission type measurements have been chosen, rather than reflection, because the former method gives one more parameter (thickness) for comparisons between experiments and theory. The energy loss suffered by primary electrons is obtained from published experimental results of electron scattering by thin foils. The escape of low energy electrons $(.25$ to $7.5 \mathrm{eV})$ is studied in detail using time dependent perturbation theory. The resulting transport problem is solved with the exact scattering probability distribution by a Monte Carlo method. It is shown that for low energy electrons generated isotropically inside a solid, an exponential probability of escape exists. Escape parameters and energy losses are computed for $\mathrm{CsI}, \mathrm{KCl}, \mathrm{NaF}$, and $\mathrm{LiF}$, representing alkali halides from the highest to lowest $\mathrm{Z}$.

The experimental yield of films of those four materials, for thicknesses of 125 to $1000 \AA$, has been measured by a pulsed method. The energy distribution of emitted electrons for the same materials has also been measured, making certain that the films remained uncharged. A Kelvin probe was used to establish that fact and also to determine a true zero of contact potential difference between collector and emitter.

A mathematical model of secondary emission based on the detailed theoretical results is proposed and found to agree well with the experiments. Approximate internal excitation functions and composite escape functions for secondaries are computed.
\end{abstract}


The results show that perturbation theory gives good quantitative values of escape parameters and that secondary emission from alkali halides is mainly determined by the energy losses suffered by primaries and by the electronphonon interaction of the internal secondaries. A high atomic number is favorable for both mechanisms and the difference between the yields of extreme materials like CsI and LiF is quite understandable. 


\section{INTRODUCTION}

The subject of Secondary Electron Emission has received considerable attention since it was first observed in 1902 by Austin and Starke. ${ }^{1}$ Excellent review articles have appeared in the literature, principally those by McKay, ${ }^{2}$ Bruining, 3 Kollath, ${ }^{4}$ and Dekker, ${ }^{5}$ covering the great wealth of experimental information as well as the somewhat limited theoretical understanding until 1958. Since that date, contributions by Kanter, ${ }^{6,7,8}$ and by Kanter and Sternglas ${ }^{9}$ have helped considerably in the understanding of some fundamental concepts, although relatively little work has been reported on the subject for the last few years.

Interest in the study of Transmission Secondary Emission from thin films has arisen more recently on account of the favorable planar geometry of the electron multipliers which can be made with transmission dynodes, in particular for image devices and fast photomultipliers. Dynodes made with alkali halides are especially interesting because of the high secondary emission gains obtainable from films evaporated in vacuum, and even more from low density films evaporated in an atmosphere of an inert gas. As examples, the work by Goetze, Boerio and Green ${ }^{10}$ on low density KCl films, by Emberson, Todkill and Wilcock $^{11}$ on normal density films, and by Garwin and Edgecumbe ${ }^{12}$ on low density $\mathrm{KCl}$ films for the detection of multi-MeV electrons in High Energy Physics, can be mentioned.

The high secondary gain properties of low density insulator films are very closely related to the trapping of charge and the building up of very high fields within the film. ${ }^{10,13}$ A detailed study of the basic processes involved can not be undertaken unless the phenomenon of secondary emission from simpler films, i. e., normal density, is understood much better than it is to date. It is the 
purpose of this report to present the work carried out at SLAC toward the understanding of the basic phenomena governing the secondary emission of alkali halide films of normal density under electron bombardment in transmission.

Certainly, reflection and transmission secondary emission are only two different aspects of the same phenomenon, but the study of secondary emission in transmission offers one advantage over reflection and that is the ability, in transmission, of limiting in effect the depth of penetration of primary electrons by changing film thickness. Thus one more parameter is available for the final comparison of theory and experiment and the study can be made more complete. Chapter I discusses the theory of secondary emission applicable to alkali halides and, in particular, it studies the rate of energy loss suffered by primary electrons and the escape mechanism of low energy electrons as governed by the electron-phonon interaction. Chapter II covers the measurement of transmission secondary emission yield of thin films of four representative alkali halides, and the measurement of energy distribution of emitted secondaries for the same materials. Finally, Chapter III presents a comparison between theoretical and experimental results showing that the theory is essentially correct, not only qualitatively but also quantitatively, with the possible exception of the escape characteristics of very low energy electrons moving in a crystal of alkali halide with low average atomic number. 


\section{Chapter I}

\section{THEORY}

\section{A. Preliminary Considerations}

It has been traditional to divide the problem of secondary emission into two parts: (a) the loss of energy suffered by the primary electrons penetrating a solid, with the consequent excitation of electrons to energies above the vacuum level of the material, and (b) the energy loss mechanisms of conduction electrons, determining the fraction of excited electrons which are actually able to leave the solid.

As observed by Dekker, ${ }^{5}$ the separation into two separate problems may not be very meaningful for the case of metals and narrow gap $(\approx 1 \mathrm{eV})$ semiconductors. For a large range of energies within the allowed bands, electrons (primaries or secondaries) can be scattered in a sort of continuous cascade until they either are emitted or become thermalized. The separation between processes (a) and (b) would be quite artificial then. Wolff ${ }^{14}$ has studied a cascade process for metals as a solution to both parts (a) and (b) when the primary energy is small $(<100 \mathrm{eV})$, although for more energetic primaries he has to take some plausible distribution of initial secondary energies, $\mathcal{E}<100 \mathrm{eV}$, as a solution to part (a). By the use of a screened Coulomb interaction between electrons, he is able to predict rather well the energy distribution and total yield of secondaries from monovalent metals.

In the case of insulators, however, the separation into two problems seems considerably more reasonable. Neglecting excitons and lattice imperfections at first, electrons whose energies are above the conduction band edge by an amount less than the energy gap cannot suffer further inelastic collisions with valence band electrons, as that would result in placing at least one electron in the forbidden region. The presence of lattice imperfections and impurities may allow a certain number of 
such transitions, until, for a constant number of traps, some equilibrium occupancy of trapping states is reached. The absorption of electrons in the exciton formation process appears to be demonstrated by the drop in SE coefficient with primary electron energies near the first optical absorption band. 5,15 Due to the ability to generate excitons, one should then restrict the range of energies over which an electron cannot suffer further inelastic collisions with electrons to $\mathscr{G} \approx \mathcal{E}_{1}$, where $\mathscr{E}_{1}$ is the energy of the first fundamental absorption band $\left(\mathscr{E}=0\right.$ at the conduction band edge). Dekker ${ }^{16}$ has analyzed theoretically the possibility that excitons generated by bombardment with primary electrons may lead to an enhancement of secondary emission, in the same manner as optically generated excitons result in photoemission from F-centers, as reported by Apker and Taft. ${ }^{17}$ Dekker $^{5}$ reports at a later date that experiments on MgO, with F-center concentrations of up to $5 \times 10^{18} \mathrm{~cm}^{-3}$, did not reveal any yield dependence on color center density and give some plausibility arguments for this result. (At about the same $\mathrm{F}$-center concentration, however, the yield is known to start decreasing. ${ }^{18}$ )

Thus, except for cases with large concentrations of defects affecting the secondary yield, it appears plausible to define some distribution, non-zero for $0<\mathcal{E}_{0} \lesssim \mathcal{E}_{1}$, which is a function of primary energy $E_{p}$, of position within the film, and of material, representing the number of electrons generated between energies $\mathcal{E}_{0}$ and $\mathcal{E}_{0}+\mathrm{d} \mathcal{E}_{0}$ per unit energy loss suffered by the primary beam. This is the outcome of solving problem (a). From part (b) one would want to obtain a set of probabilities of escape for the generated secondaries. This approach will be followed in the work to be reported. 
B. Relationship Between the Energy Loss Suffered by Primary Electrons and the Generation of Secondaries

All the existing theories of secondary emission have assumed a simple proportionality between the energy loss suffered by a primary electron in a certain thickness of material and the number of secondaries generated in the same region of space. This proportionality has finally been proven experimentally by Kanter ${ }^{7}$ by differentiating with respect to thickness his results for transmission of thin films. He shows that, at sufficiently high primary energies for negligible backscattering losses, the secondary yield in transmission for $\mathrm{Al}$ and for $\mathrm{C}$ films is proportional to the energy loss at the exit side of the film. He finds that the average energy dissipation density required to obtain one escaping electron is approximately $100 \mathrm{eV} /\left(\mu \mathrm{g} / \mathrm{cm}^{2}\right)$ for $\mathrm{Al}$ and $210 \mathrm{eV} /\left(\mu \mathrm{g} / \mathrm{cm}^{2}\right)$ for carbon.

C. The Loss of Energy by Electrons Below $12 \mathrm{keV}$

1. Comments on Published Theoretical Results

Phenomenological theories in which primary electrons are postulated to lose energy in accordance with some power law are described in the review articles. $^{2-5}$ For reflection secondary emission, Baroody ${ }^{19}$ found that a properly normalized graph can represent the yield characteristics of a large number of solids. Several degrees of agreement have been found between experimental values of yield and the power law theories, depending on the proper choice of the exponent " $n$ " in expressions of the form

$$
\frac{\mathrm{dE}}{\mathrm{dx}} \propto \frac{1}{\mathrm{E}^{\mathrm{n}}} .
$$

Quantum mechanical theories have focused their attention on the Coulomb scattering between a primary electron and electrons in the solid. The primary electron with wave function $\psi(\vec{R})=e^{i(\vec{K} \cdot \vec{R})}$ and energy $\hbar^{2} K^{2} / 2 m$ interacts 
with electrons in the solid represented by Bloch states $\psi_{\vec{k}}(\vec{r})=u_{\vec{k}}(\vec{r}) \exp [i(\vec{k} \cdot \vec{r})]$ by means of Coulomb potential

$$
V(\vec{R}, \vec{r})=\frac{e^{2}}{|\vec{R}-\vec{r}|} \exp [-\lambda|\vec{R}-\vec{r}|] \text {. }
$$

The screening factor was first introduced into this problem by van der Ziel, ${ }^{20}$ while previous work by Wooldridge, ${ }^{21}$ and Dekker and van der Ziel ${ }^{22}$ used unscreened Coulomb potentials. By expanding $u_{\vec{k}}(\vec{r})$ in a series

$$
u_{\vec{k}}(\vec{r})=\sum_{\vec{n}=0}^{\infty} C_{\vec{n}}(\vec{k}) \exp [i(\vec{n} \cdot \vec{r})]
$$

where $\vec{n}$ are reciprocal lattice vectors, the rate of transitions from state $(\overrightarrow{\mathrm{K}}, \overrightarrow{\mathrm{k}})$ to all possible states $\left(\overrightarrow{\mathrm{K}^{\prime}}, \overrightarrow{\mathrm{k}}^{\prime}\right)$ is computed for three particular cases: ${ }^{20}$

(a) Weakly bound lattice electrons; interactions in which the lattice takes up momentum differences (Umklapp process). It corresponds to Wooldridge's ${ }^{21}$ theory (except for screening) and can apply to metals.

(b) Weakly bound lattice electrons; both energy and momentum are conserved by the colliding electrons alone; corresponds to Baroody's 19 free electron theory, applicable to metals.

(c) Strongly bound electrons, $u_{\vec{k}}(\vec{r})$ is highly localized, as it would be in insulators. Van der $\mathrm{Ziel}^{20}$ shows that screening effects can be neglected in this case, and the results by Dekker and van der $\mathrm{Ziel}^{22}$ should be applicable to the case of insulators.

Case (c) gives as a result for energy loss the expression

$$
-\frac{d E}{d x}=\frac{2 \pi e^{4}}{E} N_{a} Z^{\prime} \log \frac{2 E}{E_{i}^{!}}
$$

where $E$ is the energy of the electron, $\mathrm{N}_{\mathrm{a}}$ is the number of atoms per unit volume, $Z^{\prime}$ is the number of electrons per atom which contribute to energy loss, and $E_{i}^{\prime}$ 
is an average ionization energy for the atoms, which is a slowly varying function of $\mathrm{E}$. It is not clear, however, whether Dekker and van der Ziel were thinking of the case in which the ability of the primary electrons to ionize the atomic inner shells decreases as $\mathrm{E}$ decreases. If they were, the representation of inner core electron states by Bloch functions appears questionable.

Equation (1) is practically identical to the well-known result by Bethe ${ }^{25}$ for the energy loss suffered by electrons passing through matter. In the form used by Lane and Zaffarano ${ }^{26}$ and by Young ${ }^{27}$ to find the penetration of electrons in matter it becomes

$$
-\frac{\mathrm{dE}}{\mathrm{dx}}=\frac{1.3018 \times 10^{-19} \mathrm{~N}}{\mathrm{E}} \log \left(\frac{1.166 \mathrm{E}}{\overline{\mathrm{I}}}\right)\left(\frac{\mathrm{keV}}{\mathrm{cm}}\right)
$$

where $\mathrm{N}=$ Number of electrons per cc.,$\overline{\mathrm{I}}=$ average ionization energy of atomic electrons, in $\mathrm{keV}$, and $\mathrm{E}$ is the energy of the electron, also in keV. In this case, $I$ is a constant since the intended usefulness of the expression is for a range of $E$ in which a fixed number of atomic electrons can participate in the scattering.

Two main questions arise about the usefulness of expressions (1) and (2) for primary energies of the order of $10 \mathrm{keV}$ and lower. The first one is on the decision of chosing between the two equations: Since the range of energies of interest is in the region in which the probability of ionization of inner shell electrons may go to zero, the use of Eq. (2) seems out of the question. In fact, it is shown below that Eq. (2) cannot be used at the present low energies. On the other hand, the calculation of $E_{i}^{\prime}$ in Eq. (1) from first principles is at the present time an uncertain task. The definition of $\mathrm{E}_{i}^{(2 \%)}$ is

where

$$
\mathrm{Z}^{\prime} \log \mathrm{E}_{\mathrm{i}}^{\prime}=\sum_{\mathrm{k}^{\prime}} \sum_{\overrightarrow{\mathrm{k}}} \mathrm{f}_{\overrightarrow{\mathrm{k}} \overrightarrow{\mathrm{k}}^{\prime}} \log \mathrm{E}_{\mathrm{kk}}
$$

$$
\mathrm{f}_{\overrightarrow{\mathrm{k}} \overrightarrow{\mathrm{k}}^{\prime}}=\frac{2 \mathrm{~m}}{\hbar^{2}} \mathrm{E}_{\mathrm{k} \mathrm{k}^{\prime}}\left|\mathrm{L}_{\overrightarrow{\mathrm{k}}} \overrightarrow{\mathrm{k}}^{\prime}\right|^{2}
$$


in which $\left|\mathrm{L}_{\mathrm{kk}^{\prime}}\right|^{2}$ is the optical transition probability, and $E_{\mathrm{kk}}$, is the difference in energy between state $k^{\prime}$ and $k$. The main basic difficulty which occurs immediately is that is is not clear at present whether valence electrons are in a band at all in ionic crystals. Instead, it appears that their wave functions are bound states, in which case a description by Bloch states is inadequate. Some evidence for this is to be found, for example, in the fact that in mixtures of $\mathrm{KCl}$ and $\mathrm{KBr}$ the absorption bands of both materials appear together, instead of the mixture resulting in bands in intermediate positions. ${ }^{28}$

Sternglass' semiphenomenological theory, ${ }^{23}$ which has been very successful in explaining reflection secondary emission, takes as a basis the idea that in all materials the primary electrons interact not only with outer shell electrons but also with the inner atom shells, as long as the primary electrons have sufficient energy to ionize electrons of different shells. From this consideration the individual atoms need to be regarded as determining the energy loss of primaries and the relationship between energy loss and the number of secondaries generated. His theory of secondary emission uses an expression similar to Eq. (1) in which the value of $E_{i}^{\prime}$ is left as part of an adjustable parameter, $\beta$, dependent on $\mathrm{E}_{\mathrm{p}}$. As he shows, and is well supported by Jahrreiss ${ }^{24}$ for reflection secondary emission, the parameter $\beta$ changes value in sharp breaks at energies corresponding to know $\mathrm{x}$-ray lines of the secondary emitter. From his theory, Sternglass determines the average energy $\bar{E}_{0}$ expended by the primary electron per internal secondary generated. He finds $\overline{\mathrm{E}}_{0}$ to range between $20 \mathrm{eV}$ for atoms with heavily populated valence $\mathrm{p}$ or $\mathrm{d}$ shells to $35 \mathrm{eV}$ for atoms with a simple electron in the outermost shell. It is necessary to emphasize that $\overrightarrow{\mathrm{E}}_{0}$ is an empirical value obtained from fitting Sternglass' theory to experimental results and its actual magnitude depends on some assumptions made about the trans mission characteristics of the exit potential barrier. For the many important details of Sternglass' theory the reader is directed to the original paper. ${ }^{23}$ 
The second question has to do with multiple scattering of the primary electrons. As the primary electron energy is reduced, large angle collisions will result in energy losses per unit length in the initial direction of the primary electron which are quite different from $\mathrm{dE} / \mathrm{dx}$ in the direction of the electron path. This fact is also recognized by Sternglass. ${ }^{23}$ In the process of finding the mean depth of penetration of primary electrons $\bar{d}$, he changes the mean free path $\lambda_{\mathrm{sp}}$ into the transport mean free path $\lambda_{\text {tp }}$ of diffusion theory

$$
\lambda_{\text {tp }}=\lambda_{\mathrm{sp}}(1-\overline{\cos \theta})^{-1} \text {, }
$$

where $\overline{\cos \theta}$ is the mean cosine of the angle of scattering associated with $\lambda_{\mathrm{sp}}$. After defining $\vec{d}$, Sternglass assumes that all internal secondaries are generated at that depth and uses an empirical exponential escape depth to account for their probability of escape.

For transmission secondary emission, Jahrreiss ${ }^{24}$ has shown that a point source of electrons at a mean depth $\overline{\mathrm{d}}$, which works so well in reflection, will not be good enough for good detailed results. The reason can be made quite clear by considering the extreme case of a film of thickness less than $\bar{d}$. The secondary yield would become zero. For transmission, therefore, he proposes a function $\mathrm{W}(\mathrm{x})$ proportional to the probability of creation of secondary electrons of the form

$$
\begin{aligned}
& W(x)=W(d) \exp \left[-\alpha_{1}(d-x)\right] \text { for } x \leq d \\
& W(x)=W(d) \exp \left[-\alpha_{2}(x-d)\right] \text { for } d<x,
\end{aligned}
$$

where $d$ is equivalent to Sternglass' $\vec{d}$. A development from this assumption allows Jahrreiss to extend Sternglass' theory to transmission secondary emission. Comparison with experiments yields $\alpha_{1}$ and $\alpha_{2}$ among other parameters.

In spite of the understanding of the underlying phenomena which has resulted from Sternglass' contribution, none of the theories can readily be used to calculate $-\mathrm{dE} / \mathrm{dx}$ for any given material. 
More recently, Anderson, Laponsky, Peria, et $\underline{\text { al. }},{ }^{74}$ have calculated a secondary production function (proportional to $\mathrm{dE} / \mathrm{dx}$ ) by considering a Bethe type energy loss up to a certain point and then a diffusion process. Their results seem good in a comparison with the secondary emission characteristics of $\mathrm{MgO}$.

For the purpose of the present study, however, it seems safest to obtain data on the energy loss suffered by primary electrons by a treatment of the experimental results of Young, ${ }^{27}$ Kanter, ${ }^{6}$ and Kanter and Sternglass. 9

2. The Definition of Range and Bethe's Equation Young $^{27}$ measured the fraction of transmitted current vs. energy of the incoming electrons for several thicknesses of $\mathrm{Al}_{2} \mathrm{O}_{3}$ film. He defined a practical range for the electrons as the extrapolation to zero transmission of the linear part of the transmission vs. energy curves obtained experimentally. In this fashion he arrived at the expression for range $R$,

$$
R=.0115 \mathrm{E}^{1.35}, \text { for } \mathrm{R} \text { in } \mathrm{mg} / \mathrm{cm}^{2} \text { and } \mathrm{E} \text { in } \mathrm{keV} \text {. }
$$

Kanter $^{6}$ measured the fraction of transmitted electrons, $\eta_{t}$, vs. initial energy for various materials and thicknesses, as well as average and most probable exit energies ( $\mathrm{E}_{\mathrm{a}}$ and $\mathrm{E}_{\mathrm{W}}$, respectively), and average and most probable angle of scattering $\left(\theta_{\mathrm{a}}\right.$ and $\left.\theta_{\mathrm{w}}\right)$. Kanter found that defining the energy of the incoming primary electron, $E_{p}$, in terms of a critical energy, $E_{c}$, corresponding to the initial energy of an electron with range equal to the thickness of the film under investigation, all the transmission data for different materials could be presented in a compact form in one graph; likewise for the energy and angle data. The definition of range is the same as Young's, with minor differences in the numbers.

Kanter and Sternglass ${ }^{9}$ discuss the difference between the critical energy, $\mathrm{E}_{\mathrm{c}}$, defining a practical range $\mathrm{R}$ and a threshold energy, $\mathrm{E}_{\mathrm{th}}$, defining a maximum range. The latter takes into consideration that for a given thickness, 
an electron with $E_{p}=E_{c}$ actually has a finite probability of being transmitted. If $\mathrm{E}_{\mathrm{p}}$ is decreased until $\eta_{\mathrm{t}}$ goes to "zero" (within the limitations of the meas uring apparatus), the energy of the incoming electrons is then $E_{t h}$. It must be noted that electrons leaving the film with energies below $45 \mathrm{eV}$ were not being collected. For materials with $\mathrm{Z} \gtrsim 25$, it was found that $\mathrm{E}_{\mathrm{c}}=1.4 \mathrm{E}_{\text {th }}$.

In searching for an expression giving the average energy loss per unit length in the direction of the incident electron, which can be used in determining a generation rate of carriers in a transmission secondary emitter, it is clear that the experimental data of Kanter ${ }^{6}$ must be satisfied. Young ${ }^{27}$ reports good agreement between experimentally determined practical range $R$ and the results of integrating Bethe's equation[Eq. (2)]. After the above considerations this comes as a surprise, but statements about Bethe's equation being valid to very low energies are found in the literature. (For example, see Ref. 7). It was decided then to make a test between Kanter's results and an integration of Eq. (2) for a film of $1200 \mathrm{~A}$ of Al, carried out digitally by Runge-Kutta-Gill method. ${ }^{29}$

Using the value of $\overline{\mathrm{I}}=.150 \mathrm{keV}$ in Eq. (2), ${ }^{27} \mathrm{Fig} .1$ shows the average energy of the electrons (E) vs. distance into the film (x) for various energies of the incident electrons. The integration method is inherently stable and the truncation error per step is of order $h^{5}$, where $h$ is the step size. In practice, whenever this integration method is used, the errors due to truncation are kept sufficiently small by making $h$ small enough so that halving $h$ in two successive computations does not change the final step results by more than 1 part in $10^{4}$ or $10^{5}$.

Kanter's experimental results can be put into the form of average energy loss per electron in the film by noticing that

$$
\int_{0}^{x} \frac{d E}{d x} d x=E_{p}-\eta_{t} E_{a}
$$




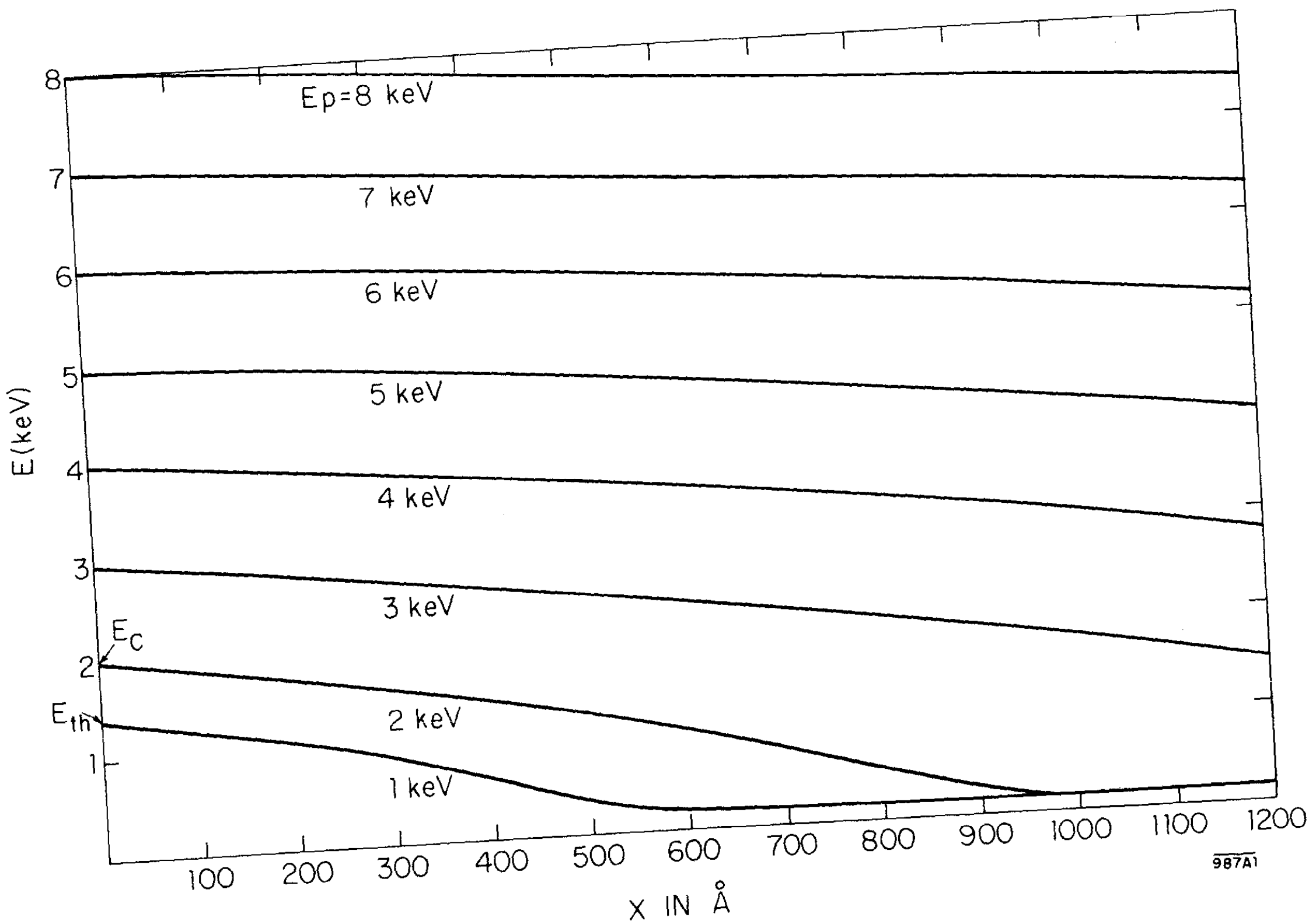

FIG. 1--Average electron energy vs. depth of penetration in a $1200 \AA \mathrm{f}$ 
where $x_{\max }$ is the thickness of the film, $E_{p}$ is the initial energy, $\eta_{t}$ is the fraction of electrons transmitted, and $E_{a}$ is the average energy of the electrons transmitted.

In Table 1 the value obtained from the integration of Bethe's equation [Eq. (2)] is compared with the right-hand side of the above equation obtained from Kanter ${ }^{6}$, and Kanter and Sternglass. 9

TABLE 1

\begin{tabular}{|c|c|c|c|c|c|}
\hline $\begin{array}{c}\mathrm{E}_{\mathrm{p}} \\
(\mathrm{keV})\end{array}$ & $-\int_{0}^{\mathrm{x}_{\max }} \begin{array}{c}\mathrm{d} \mathrm{d} \mathrm{x} \\
(\mathrm{keV})\end{array}$ & $\eta_{\mathrm{t}}$ & $\begin{array}{c}\mathrm{E}_{\mathrm{a}} \\
(\mathrm{keV})\end{array}$ & $\begin{array}{c}\mathrm{E}_{\mathrm{p}}-\eta_{\mathrm{t}} \mathrm{E}_{\mathrm{a}} \\
(\mathrm{keV})\end{array}$ & $\Delta \mathrm{E}_{\mathrm{exp}} / \Delta \mathrm{E}_{\text {Bethe }}$ \\
\hline 8 & .55 & $\sim .97$ & 6.4 & 1.8 & 3.27 \\
7 & .6 & $\sim .95$ & 5.4 & 1.88 & 3.14 \\
6 & .7 & $\sim .92$ & 4.5 & 1.87 & 2.67 \\
5 & .8 & .86 & 3.6 & 1.91 & 2.39 \\
4 & .95 & .72 & 2.6 & 2.13 & 2.25 \\
3 & 1.25 & .42 & 1.68 & 2.3 & 1.84 \\
$2=\mathrm{E}_{\mathrm{c}}$ & 1.84 & .05 & .8 & 1.96 & 1.065 \\
$1.4=\mathrm{E}_{\mathrm{th}}$ & 1.24 & $0^{(*)}$ & $0^{(*)}$ & $\sim 1.4^{(*)}$ & $\sim 1.13^{(*)}$ \\
\hline
\end{tabular}

(*) Except for electrons with $\mathrm{E}$ less than $45 \mathrm{eV}$ at the exit surface.

One can see from the table that it appears that the integral of Bethe's equation gives a discrepancy of about $6.5 \%$ for $E_{p}=E_{c}$ although from Fig. 1 it is clear that the same result would have been obtained at an energy somewhat above $2 \mathrm{keV}$. This small discrepancy by itself would seem to indicate the applicability of such an equation at these low energies. However, the discrepancies in the region $1.5 \mathrm{E}_{\mathrm{c}} \leq \mathrm{E}_{\mathrm{p}} \leq 4 \mathrm{E}_{\mathrm{c}}$ are quite substantial. At $\mathrm{E}_{\mathrm{p}}=4 \mathrm{E}_{\mathrm{c}}$, the experimental energy loss is more than three times the one given by Bethe's formula. 
Clearly, in the region between 3 and $8 \mathrm{keV}$, large angle scattering causes the true energy loss to be much larger than the loss computed from Bethe's equation. As the electron energy decreases, the failure to ionize $K$ and $L$ electrons results in a smaller true energy loss per unit length than the one given by Eq. (2). Apparently by coincidence, these two errors approximately cancel each other out when $\mathrm{E}_{\mathrm{p}} \simeq \mathrm{E}_{\mathrm{c}}$.

The above discussion does not include a consideration of backscattering, but its effects can be computed readily for the specific case of a $500 \stackrel{\circ}{\mathrm{A}}$ film of aluminum. Kanter ${ }^{8}$ gives the measured fraction of backscattered electrons $\eta$ vs. $\mathrm{E}_{\mathrm{p}}$ for such a film. Also, he reports that the average energy of the backscattered electrons is approximately half the initial energy. It is apparent from his study of the contribution of backscattered electrons to secondary emission yield that this average energy $\mathrm{E}_{a b s} \simeq 1 / 2 \mathrm{E}_{\mathrm{p}}$ holds quite well for thin films. Then we can find, for an electron with $E_{p}=4 \mathrm{keV}$, for example, how much energy is backscattered on the average. This will be $\eta \mathrm{E}_{\text {abs }}=.065 \times 2=.13 \mathrm{keV}$. Then $E_{p}-\left(\eta_{t} E_{a}\right)-\left(\eta E_{a b s}\right)=$ energy loss in the film. For a $500 \stackrel{\circ}{A}$ film,

$$
\begin{aligned}
& \mathrm{E}_{\mathrm{c}}=1 \mathrm{keV} \\
& \mathrm{E}_{\mathrm{p}}=4 \mathrm{keV} \\
& \eta_{\mathrm{t}}=.97 \\
& \mathrm{E}_{\mathrm{a}}=.8 \times 4=3.2 \mathrm{keV}
\end{aligned}
$$

or energy loss in the film $=4-(.97 \times 3.2)-.13=4-3.1-.13=.77 \mathrm{keV}$. Thus, the backscattered energy is only a fraction $.13 / .77=.17$ of the energy lost in the film. From the integration of Bethe's equation (Fig. 1), one can see that

$$
\left.\int_{0}^{500 \AA} \frac{\mathrm{d}}{\mathrm{dx}} \mathrm{dx}\right|_{\mathrm{E}_{\mathrm{p}}=4 \mathrm{keV}} \simeq .4 \mathrm{keV}
$$

and the discrepancy between true energy absorbed and the integral of Eq. (1) is 
still too large. We must conclude, therefore, that Bethe's equation does not hold in the region of $\mathrm{E}<10 \mathrm{keV}$ in spite of some coincidences.

3. Finding the Rate of Energy Loss From Experimental Results

Using the experimental results of Kanter, ${ }^{6}$ it is possible to find approximate curves for $\mathrm{dE} / \mathrm{dx}$ vs. $\mathrm{x}$ by dividing a film into a number of slabs and computing successively the contribution to energy loss by each slab. No backscattering is assumed at first. For a given thickness of remaining slabs, $E_{c}$ is found by a convenient form of the Energy-Range relation

$$
E_{c}=\left(\frac{\mathrm{R}^{\mu \mathrm{g} / \mathrm{cm}^{2}}}{13.5}\right) \cdot 77(\mathrm{keV})
$$

obtained empirically from the results presented by Kanter. This expression is somewhat different in numbers from Young's. ${ }^{27}$ The fraction of transmitted electrons $\eta_{t}$ as a function of reduced initial energy $E_{p} / E_{c}$, for films of thickness $\geq 15 \mu \mathrm{g} / \mathrm{cm}^{2}$, as given by Fig. 4 of Ref. 6, can be represented very well in the energy range of $1 \leq \mathrm{E}_{\mathrm{p}} / \mathrm{E}_{\mathrm{c}} \leq 5$ by the expression

$$
\eta_{\mathrm{t}}=\left[1-\exp \left(-\frac{\gamma-1}{\gamma^{\prime}}\right)\right]
$$

where $\gamma=\mathrm{E}_{\mathrm{p}} / \mathrm{E}_{\mathrm{c}}$ and $\gamma^{\prime}$ is a linear function of atomic number $\mathrm{Z}$ :

$$
\gamma^{\prime}=.45+.025 \mathrm{Z} \text {. }
$$

The fractional average energy $\mathrm{E}_{\mathrm{a}} / \mathrm{E}_{\mathrm{p}}$ of transmitted electrons as a function of $\gamma$, as given by Fig. 8 of Ref. 6 , is fitted again quite well by an exponential in the region $1 \leq \gamma \leq 6$

$$
\frac{\mathrm{E}_{\mathrm{a}}}{\mathrm{E}_{\mathrm{p}}}=.45\left[1-\exp \left(-\frac{\gamma-1}{1.33}\right)\right]+.4
$$

For $\gamma>6$, the above expression approaches .85. This cannot be a good representation at large $\gamma$, in which case $\mathrm{E}_{\mathrm{a}} / \mathrm{E}_{\mathrm{p}}$ should approach almost unity. Since Kanter's results are not extended to such regions, the calculation to be presented will be in error whenever $\gamma \approx 6$. 
By using the above expressions, it is possible to write a simple computer program which will find the average energy loss suffered by a beam of electrons of primary energy $E_{p}$, with a density of 1 electron $/ \mathrm{cm}^{2}$ per unit time. For the purpose of illustration consider a film of Al, with a thickness of $100 \mu \mathrm{g} / \mathrm{cm}^{2}$, divided into 10 slabs. A beam of $6 \mathrm{keV}$ electrons enters normally at $\mathrm{x}=0$. The energy loss in traversing the 10 slabs is found to be $5.1 \mathrm{keV}$. Table 2 gives all the significant numbers of the computation. Next, we assume that the last (at exit side) slab does not exist. The energy lost by the beam is $4.77 \mathrm{keV}$, giving $-(\mathrm{dE} / \mathrm{dx})=(5.1-4.77) / 10=.033\left(\mathrm{keV}-\mathrm{cm}^{2}\right) / \mu \mathrm{g}$. The same procedure is repeated until all the slabs have been eliminated. Figure 2 shows the energy lost by the beam of $6 \mathrm{keV}$ electrons from the calculations of Table 2 .

TABLE 2

\begin{tabular}{|c|c|c|c|c|c|c|}
\hline $\begin{array}{l}\text { Thickness } \\
\text { after } \mathrm{x}=0 \\
\left(\mu \mathrm{g} / \mathrm{cm}^{2}\right)\end{array}$ & $\begin{array}{c}\mathrm{E}_{\mathrm{c}} \\
(\mathrm{keV})\end{array}$ & $\gamma$ & $\eta_{\mathrm{t}}$ & $\mathrm{E}_{\mathrm{a}} / \mathrm{E}_{\mathrm{p}}$ & $1-\eta_{\mathrm{t}} \mathrm{E}_{\mathrm{p}}$ & $\begin{array}{c}\mathrm{E}_{\text {loss }} \\
(\mathrm{keV})\end{array}$ \\
\hline 100 & 4.67 & 1.28 & .30 & .48 & .851 & 5.1 \\
90 & 4.31 & 1.39 & .39 & .51 & .79 & 4.77 \\
80 & 3.93 & 1.52 & .49 & .54 & .73 & 4.38 \\
70 & 3.55 & 1.69 & .58 & .58 & .65 & 3.94 \\
60 & 3.15 & 1.90 & .68 & .62 & .57 & 3.43 \\
50 & 2.74 & 2.19 & .78 & .66 & .47 & 2.86 \\
40 & 2.31 & 2.59 & .87 & .71 & .37 & 2.25 \\
30 & 1.85 & 3.24 & .94 & .76 & .27 & 1.65 \\
20 & 1.35 & 4.43 & .98 & .81 & .19 & 1.16 \\
10 & .79 & 7.56 & .99 & .84 & .15 & .92 \\
\hline
\end{tabular}

The inadequacy of Eq. (3) at higher values of $\gamma$ becomes quite evident in Fig. 2: For $\mathrm{x}$ approaching zero, the energy loss should go to zero. A correction 


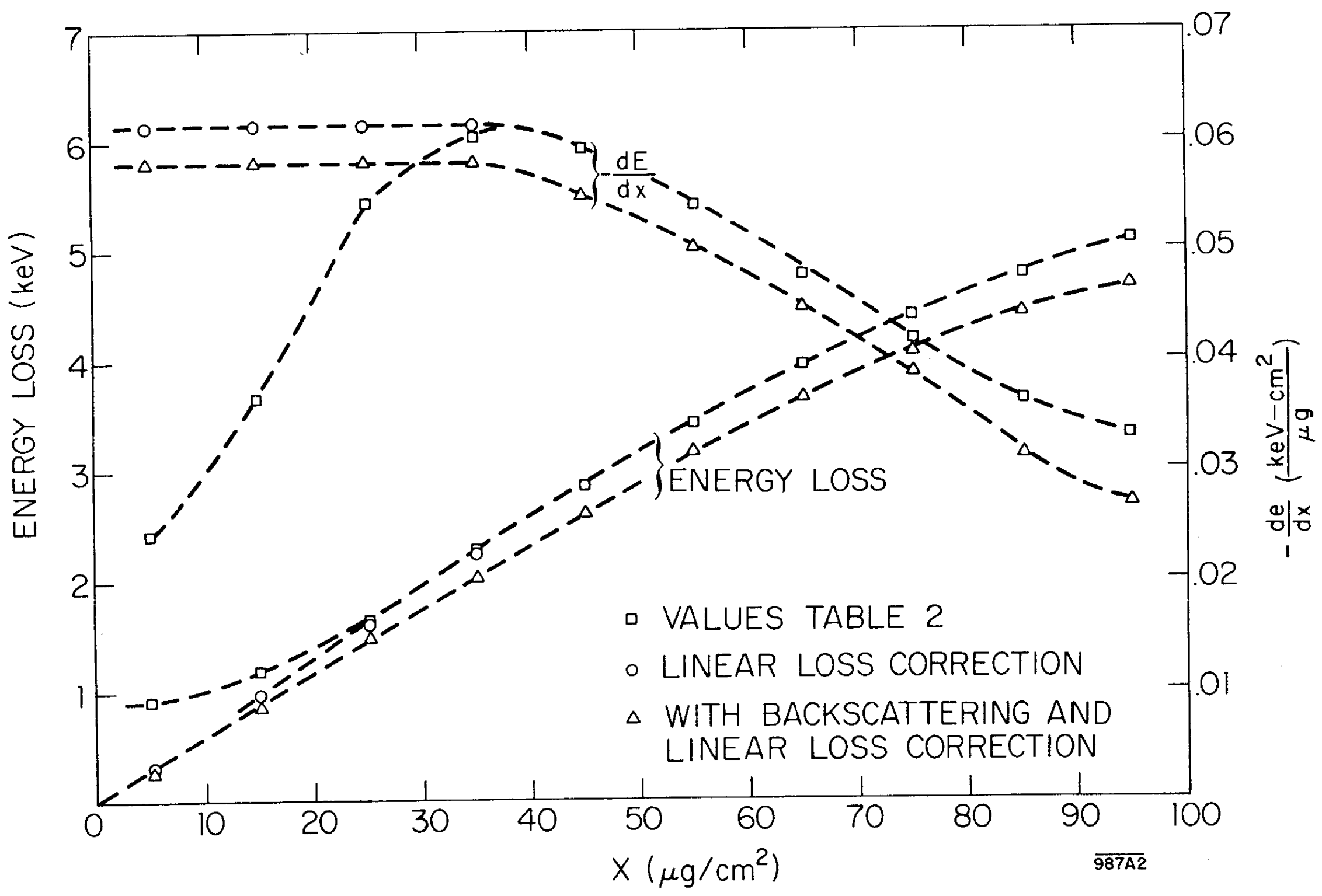

FIG. 2--Energy loss and its derivative for an electron beam of $\mathrm{E}_{\mathrm{p}}=6 \mathrm{keV}$ in aluminum 
is proposed in which a linear energy loss is suffered by the electrons up to a thickness such that $-\mathrm{dE} / \mathrm{dx}$, as calculated above, matches the slope of the linear loss. This correction is also shown in Fig. 2. If on physical grounds one excludes the possibility of discontinuities and of more than one maximum in $\mathrm{dE} / \mathrm{dx}$, the linear loss approximation is the only possible solution for the several calculations carried out for different materials.

The corrections for backscattering can be applied at this point. The energy loss computed above includes the energy not transmitted because it is backscattered by the film. This backscattered energy does not contribute (to first order) to the generation of electrons. A correction is, therefore, necessary. One finds the first difficulty in trying to specify a backscattering coefficient $\eta$ for thin films as a function of energy of the incident electron. Halliday and Sternglass ${ }^{30}$ show $\eta$ vs. atomic number for bulk materials. More information is available in a paper by Gomoyunova and Letunov. ${ }^{31}$ The former work also shows that $\eta$ holds for thin films as long as the incident electrons are of an energy $E_{p} \leq 2 E_{c}$ for the thickness of the film. For higher $E_{p}$, the only available data are for a $500 \AA$ film, given by Kanter. ${ }^{8}$ As a reasonable approximation, one can form a function $\eta\left(E_{p}\right)$ which is proportional to the one for Al when the energy has been normalized by $E_{c}$ for a given film thickness. The proportionality constant is then found by requiring that at low $\mathrm{E}_{\mathrm{p}}, \eta=\eta_{\text {bulk }}$. This function can be represented by

$$
\begin{aligned}
\eta & =\eta_{\text {bulk }} & \text { for } & \gamma \leq 2 \\
& =\eta_{\text {bulk }} \exp \left(-\frac{\gamma-2}{3.75}\right) & \text { for } & \gamma \geq 2
\end{aligned}
$$

which fits Fig. 2 of Ref. 8 quite well up to $\gamma \approx 8$.

The procedure for the calculation is similar to the previous one. Consider first the 10th slab. The primary electron which started at $6 \mathrm{keV}$ reaches the 10 th slab with an average energy of $\approx .89 \mathrm{keV}$ (From Table 2). The average angle of 
arrival is not zero, but this will be disregarded given the approximate nature of the correction. Since the average energy of backscattered electrons is approximately one-half the energy of the bombarding electrons, ${ }^{8}$ the energy backscattered by the last slab can be computed. Next one takes the 9 th and 10 th slabs and the procedure is repeated until the total energy backscattered is found. The correction to the total energy loss up to the slab at $x_{i}$ is equal to total backscattered energy minus the energy backscattered by the material at $x>x_{i}$.

Table 3 shows the corrections for the case of $6 \mathrm{keV}$ primary electrons and $100 \mu \mathrm{g} / \mathrm{cm}^{2}$ of Al considered above. The corrections are also shown on Fig. 2

TABLE 3

\begin{tabular}{|c|c|c|c|c|c|c|c||c|c|}
\hline $\begin{array}{r}\text { Slab } \\
\text { No. }\end{array}$ & $\begin{array}{c}\text { Thickness } \\
\mu \mathrm{g} / \mathrm{cm}^{2}\end{array}$ & $\mathrm{E}_{\mathrm{p}}$ & $\mathrm{E}_{\mathrm{c}}$ & $\gamma$ & $\eta$ & $\mathrm{E}_{\mathrm{abs}}$ & $\begin{array}{c}\text { Energy } \\
\text { Back- } \\
\text { scattered } \\
(\mathrm{keV})\end{array}$ & $\begin{array}{l}\text { Slab } \\
\text { No. }\end{array}$ & $\begin{array}{c}\text { Energy } \\
\text { Back- } \\
\text { scattered } \\
(\mathrm{keV})\end{array}$ \\
\hline 10 & 10 & .89 & .79 & 1.12 & .15 & .45 & .067 & $1-10$ & .42 \\
$9-10$ & 20 & 1.22 & 1.35 & .91 & .15 & .61 & .092 & $1-9$ & .36 \\
$8-10$ & 30 & 1.61 & 1.84 & .87 & .15 & .80 & .12 & $1-8$ & .33 \\
$7-10$ & 40 & 2.05 & 2.31 & .89 & .15 & 1.02 & .15 & $1-7$ & .30 \\
$6-10$ & 50 & 2.56 & 2.74 & .93 & .15 & 1.28 & .19 & $1-6$ & .27 \\
$5-10$ & 60 & 3.13 & 3.15 & .99 & .15 & 1.56 & .23 & $1-5$ & .23 \\
$4-10$ & 70 & 3.77 & 3.55 & 1.06 & .15 & 1.88 & .28 & $1-4$ & .19 \\
$3-10$ & 80 & 4.41 & 3.93 & 1.12 & .15 & 2.20 & .33 & $1-3$ & .14 \\
$2-10$ & 90 & 5.04 & 4.31 & 1.17 & .15 & 2.52 & .37 & .695 \\
$1-10$ & 100 & 5.68 & 4.67 & 1.21 & .15 & 2.84 & .42 & 1 & .047 \\
\hline
\end{tabular}

Also shown on Fig. 2 are the values of $-d E / d x$ from the above calculations. It can be seen that the backscattering correction is not of great significance in this particular case; however, for higher $\mathrm{Z}$ materials the correction is more substantial. 
Figure 3 shows $-\mathrm{dE} / \mathrm{dx}$ for several values of $\mathrm{E}_{\mathrm{p}}$, as computed for $100 \mu \mathrm{g} / \mathrm{cm}^{2}$ of $\mathrm{Al}$ by division into 100 slabs for better accuracy. The shift of the maximum of energy loss per unit thickness and the decay of the function at both sides of the maximum gives substantial support to Sternglass' theory, as modified by Jahrreiss. ${ }^{24}$ Results with and without the linear loss correction are given. Although the linear loss correction appears necessary as indicated above, the corrected results of Fig. 3 seem somewhat peculiar. A more uniform decay as one moves from the peak towards low values of $\mathrm{x}$ makes better physical sense from the point of view of a spreading shower of electrons starting at $x=0$. Without applying the correction, however, $-\mathrm{dE} / \mathrm{dx}$ becomes too small at low $\mathrm{x}$. It seems that the physical process would be described better by an intermediate situation. This is confirmed by making a comparison of the values of $-\mathrm{dE} / \mathrm{dx}$ from Fig. 3 at the exit of $\mathrm{Al}$ films of 29.5 and $17.5 \mu \mathrm{g} / \mathrm{cm}^{2}$ with the secondary yield of such films as given by Kanter. ${ }^{7}$ This comparison is analogous to the one made by that author in Fig. 4 of Ref. 7 and the results obtained are almost as good as his. For the thicker film the agreement is better without the linear loss correction, but for thethinner film the yield at the higher energies falls between the curves found with and without the linear loss correction. Separate computations for the 100 slabs covering 29.5 and $17.5 \mu \mathrm{g} / \mathrm{cm}^{2}$ without the linear loss correction and with or without the backscattering corrections give about the same results. The most obvious difference is a somewhat different proportionality constant between $\delta$ and $-\mathrm{dE} / \mathrm{dx}$, which is found to range between 80 and $100 \mathrm{eV} /\left(\mu \mathrm{g} \cdot \mathrm{cm}^{2}\right)$.

Unfortunately, it does not seem possible to calculate the intermediate energy loss indicated above from the available data. The matter will have to be left open at this time. 


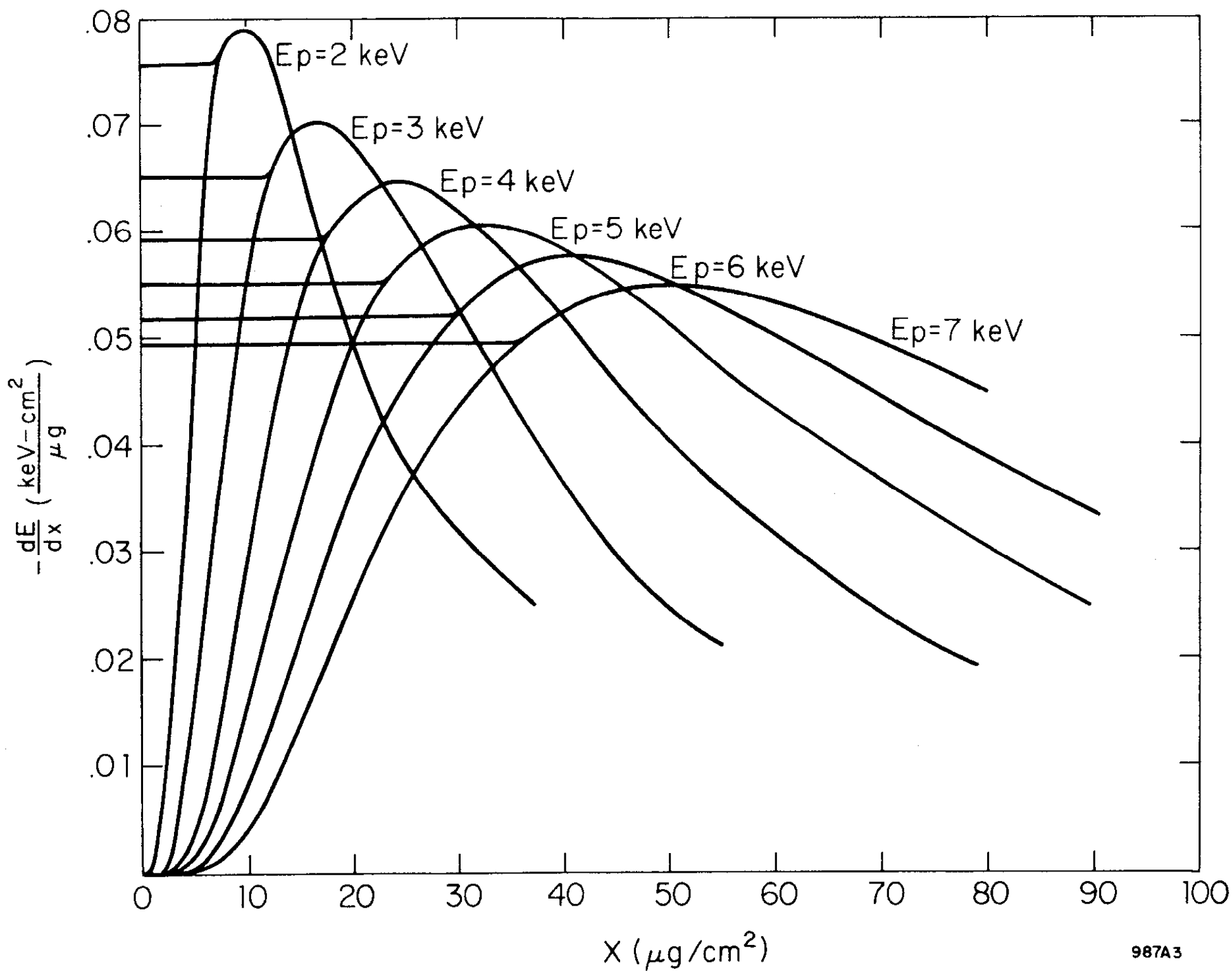

FIG. 3--Energy loss per unit thickness in a $100 \mu \mathrm{g} / \mathrm{cm}^{2}$ film of $\mathrm{Al}$, with and without the linear loss correction 


\section{Application to Composite Films}

The practical films used at the Stanford Linear Accelerator Center for the study of transmission secondary emission consist of a $1000 \AA \mathrm{Al}_{2} \mathrm{O}_{3}$ supporting film, a $500 \AA$ film of $\mathrm{Al}$ and finally an evaporated film of an alkali halide.

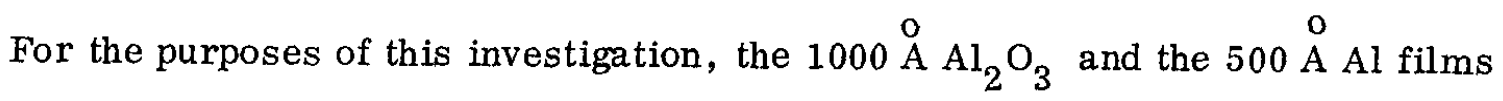
will be considered as a substrate with usually negligible secondary emission in comparison to that of the alkali halide. The energy loss suffered by the primary electrons in traversing the substrate is certainly not negligible and its presence cannot be neglected.

Since the average $\mathrm{Z}$ of $\mathrm{Al}_{2} \mathrm{O}_{3}$ is not very far from that of $\mathrm{Al}$, one can use Kanter's transmission measurements ${ }^{6}$ to find the average number of electrons transmitted by the substrate, their average energy and their average angle for a normally incident beam. The results are then used as initial values for the computation of $-\mathrm{dE} / \mathrm{dx}$ in the alkali halide film. The average angle is used to determine an effective thickness $\tau^{\prime}=\tau / \cos \theta_{\text {av }}$ where $\tau$ is the true film thickness of the alkali halide and $\theta_{\text {av }}$ is the average angle of the incoming electrons. The energy loss and backscattering corrections are computed for a film thickness $\tau^{\prime}$, but the derivative $-\mathrm{dE} / \mathrm{dx}$ is taken in the forward direction so that the final result is given in $\mathrm{keV}-\mathrm{cm}^{2} / \mu \mathrm{g}$ in the direction normal to the film surface. This method of calculation is, of course, only approximate and it is only appropriate if the electrons transmitted by the substrate have angular distribution which is independent of their energy distribution. There does not seem to be any available information to substantiate such independence.

Table 4 shows the substrate transmission data used for the calculation. They are based on $1000 \stackrel{\circ}{\AA} \mathrm{Al}_{2} \mathrm{O}_{3}=35 \mu \mathrm{g} / \mathrm{cm}^{2}, 500 \AA \mathrm{Al}=13.5 \mu \mathrm{g} / \mathrm{cm}^{2}, \mathrm{E}_{\mathrm{c}}=2.7 \mathrm{keV}$, and $\mathrm{Z} \approx 11.5$. 
TABLE 4

\begin{tabular}{|c|c|c|c|c|}
\hline $\begin{array}{c}\mathrm{E}_{\mathrm{p}} \\
(\mathrm{keV})\end{array}$ & $\mathrm{E}_{\mathrm{p}} / \mathrm{E}_{\mathrm{c}}$ & $\eta_{\mathrm{t}}$ & $\begin{array}{c}\mathrm{E}_{\mathrm{av}} \\
(\mathrm{keV})\end{array}$ & $\begin{array}{c}\theta_{\mathrm{av}} \\
\text { (degrees) }\end{array}$ \\
\hline 4 & 1.48 & .48 & 2.25 & 45 \\
5 & 1.85 & .68 & 3.2 & 43.5 \\
6 & 2.22 & .81 & 4.1 & 41.5 \\
7 & 2.59 & .88 & 5.05 & 39 \\
8 & 2.97 & .93 & 6.0 & 37 \\
9 & 3.33 & .95 & 6.9 & 35 \\
10 & 3.7 & .97 & 7.8 & 33 \\
11 & 4.07 & .98 & 8.8 & 31 \\
12 & 4.44 & .99 & 9.75 & 29 \\
\hline
\end{tabular}

Computations for two alkali halides, with quite different $\mathrm{Z}$ are shown in Fig. 4 and Fig. 5. Both computations have been carried out with 200 slabs covering a thickness $\tau=50 \mu \mathrm{g} / \mathrm{cm}^{2}$. Results with and without the linear loss correction are shown, except for the case of $\mathrm{E}_{\mathrm{p}}=12 \mathrm{keV}$, in Fig. 5. The linear loss corection can only be computed for thicknesses above a certain minimum. In a practical problem with $\tau$ less than such a minimum, the computation will be carried out for as much material as necessary but only the desired number of slabs will be taken for further handling. Error is introduced only through the backscattering correction and in view of the approximate character of the calculation, it will be neglected.

5. The Secondary Electron Generation Function

The total number of electrons generated per unit volume with energy below the one corresponding to the first exciton peak of the material considered will be taken as being proportional to $-\mathrm{dE} / \mathrm{dx}$ at the same region in the film. 


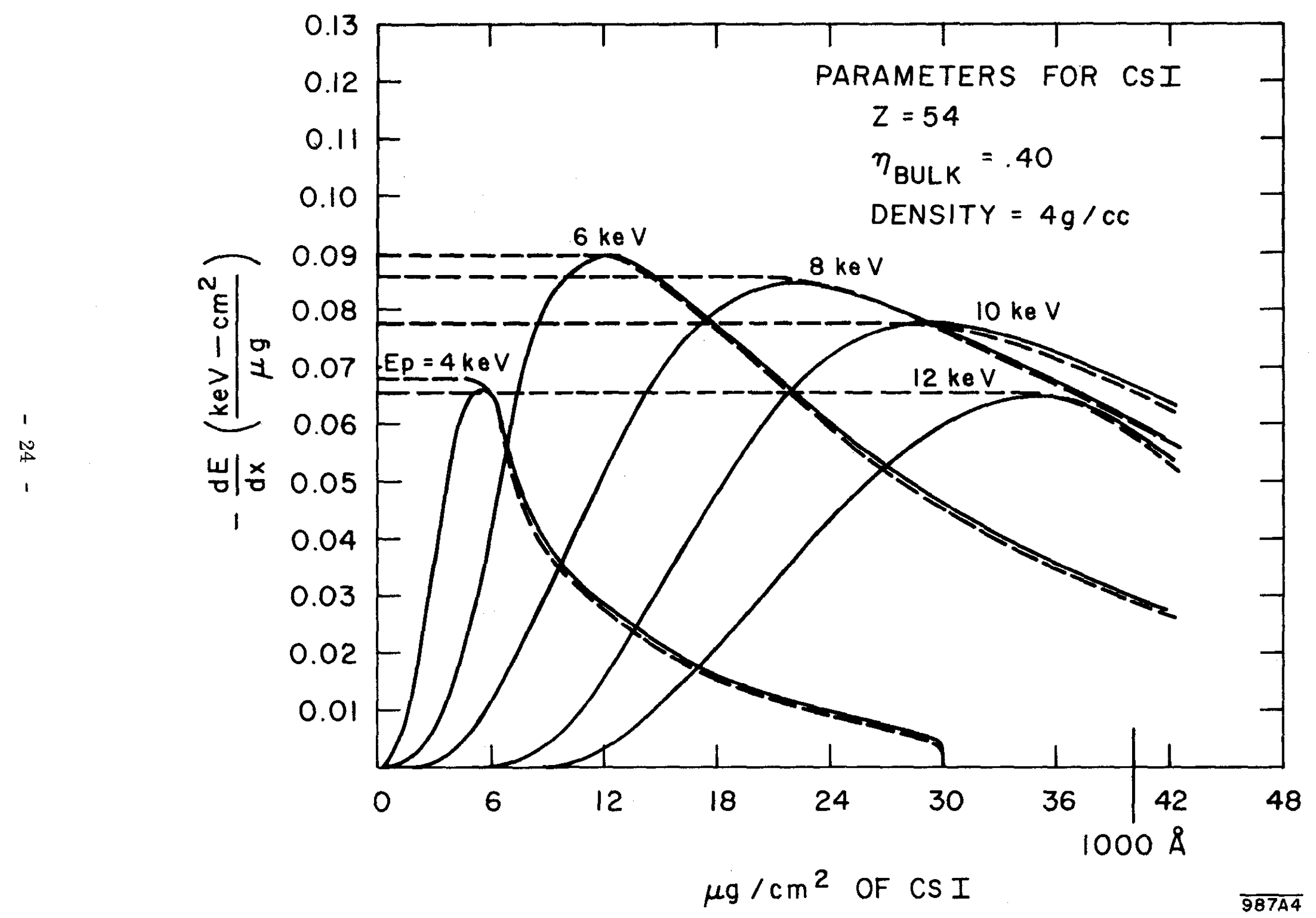

FIG. 4--Energy loss per unit length for a CsI composite film 


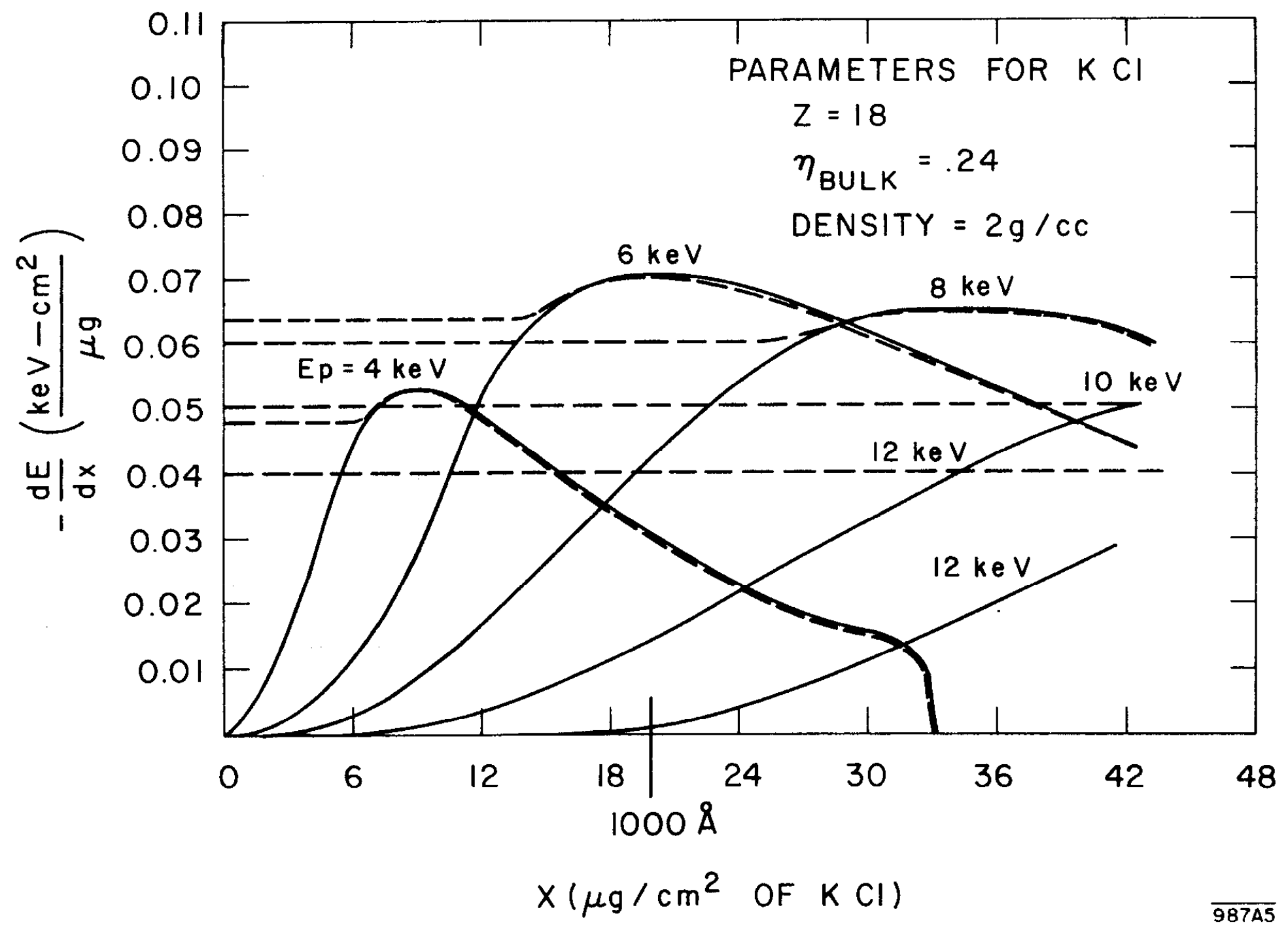

FIG. 5--Energy loss per unit length for a $\mathrm{KCl}$ composite film 
The energy distribution is unknown and will be one of the results of the comparison between experiment and theory in Chapter III. Some assumption has to be made about the angular distribution of the generated electrons. Jahrreiss 24 considers that for $\mathrm{x}$ less than the position of the maximum in $-\mathrm{dE} / \mathrm{dx}$, the generated secondaries must have a significant forward direction while beyond the peak their direction may be random. Although Jahrreiss ${ }^{32}$ has recently observed that at sufficiently high $E_{p}$ the angular distribution of slow secondary electrons from thin metal foils is forward directed, deviations from a cosine distribution do not occur until $\mathrm{E}_{\mathrm{p}} \approx 6 \mathrm{E}_{\mathrm{c}}$. Since the present investigation is limited to $4 \mathrm{keV} \leq \mathrm{E}_{\mathrm{p}} \leq 12 \mathrm{keV}$, the primary electrons still generate escaping secondaries with a cosine distribution from the substrate alone (see Table 4). Such a distribution is commonly understood to indicate a spherically symmetric internal generation function. It is then reasonable to expect that the primary electrons, after having been scattered considerably by the substrate will continue generating low energy electrons with spherically symmetric distribution, independent of their energy.

From the above considerations, and for a given material and primary energy, we consider the existence of a generation function

$$
\mathrm{G}\left(\mathscr{E}_{0}, \mathrm{x}\right) \mathrm{d} \mathscr{E} \mathrm{dx}=-\frac{\mathrm{dE}}{\mathrm{dx}}(\mathrm{x}) \mathrm{G}\left(\hat{G}_{0}\right) \mathrm{d} \xi_{0} \mathrm{dx}
$$

such that $(-d E / d x) d x$ is the energy lost by a primary beam of unity cross section in a length $d x$ and $G\left(\mathcal{E}_{0}\right) d \mathscr{E}_{0}$ is the number of internal secondary electrons generated isotropically between energies $\mathscr{E}_{0}$ and $\mathscr{G}_{0}+\mathrm{d} \mathscr{G}_{0}$ per unit energy loss suffered by the primary beam. One can think of the primary beam as having a current density of 1 electron per unit area per unit time in all the computations. 


\section{The Escape Mechanism of Secondary Electrons}

\section{Preliminary Consideration}

The interaction between electrons and the optical modes of the lattice vibrations has been recognized for a long time to be the predominant interaction controlling the transport of low energy (a few eV) electrons in pure ionic crystals. In 1937 , Fröhlich ${ }^{33}$ was able to compute the high field breakdown of ionic crys tals, with reasonable agreement with experimental results, by considering the collisions between free conduction electrons and the longitudinal optical mode lattice vibrations. In 1939 , the same author ${ }^{34}$ improved on his previous work by showing that it is possible to solve the same problem without having to compute the reduced masses and vibration frequencies of the lattice. Instead, experimentally determined dielectric constants and infrared absorption frequencies can be used, and these are easily determined even for crystals which are not fully ionic in character. Dekker ${ }^{35,36}$ has developed a simplified onedimensional random walk model based on the electron-phonon interaction theory of Fröhlich which predicts the temperature dependence of the secondary emission coefficient $\delta$ for ionic crystals. For two temperatures $\mathrm{T}_{1}$ and $\mathrm{T}_{2}$, the ratio of the yields is

$$
\frac{\delta_{1}}{\delta_{2}}=\left[\left(2 \mathrm{n}_{\nu 2}+1\right) /\left(2 \mathrm{n}_{\nu 1}+1\right)\right]^{1 / 2} \text { where } \mathrm{n}_{\nu \mathrm{i}}=1 /\left[\exp \left(\mathrm{h} \nu / \mathrm{k}_{\mathrm{i}}\right)-1\right],
$$

$\nu$ being the frequency of the longitudinal optical vibrations of the lattice. The agreement with experiment has recently been reinvestigated by Stuchiniskii ${ }^{37}$ for $\mathrm{MgO}$ at temperatures ranging from $100^{\circ} \mathrm{K}$ to $600^{\circ} \mathrm{K}$ and found to be quite good, although the experimental temperature dependence was slightly stronger than the one predicted by Dekker. Khokley and van Vliet have investigated further the escape mechanism of secondary electrons in polar crystals in the absence of an electric field, ${ }^{38}$ and with field enhancement. ${ }^{39}$. For both cases, 
the authors have set up a Boltzman transport equation based on the following simplifying assumptions :

1. The spatial dependence of the internal secondary electron distribution is one dimensional.

2. The secondary electrons can be characterized by a single isotropic effective mass $m^{*}$, with energy $\mathcal{E}=\hbar^{2} k^{2} / 2 m^{*}$ measured with respect to the bottom of the conduction band.

3. The electrons interact only with lattice vibrations (optical polar modes) which cause only strong forward scattering for $\mathscr{G} \gg \hbar \omega$ (phonon energy).

These assumptions will be reviewed carefully below, and in particular it will be shown that the assumption of only strong forward scattering is not a very good description of the electron phonon interaction.

2. The Electron-Phonon Interaction

The problem of finding a scattering rate for electrons interacting with the optical modes of an ionic lattice was initially solved by Fröhlich ${ }^{33,34}$ by timedependent perturbation theory. In that approach one uses the adiabatic principle, in which the electronic states are largely independent of the lattice vibrational states. The unperturbed wave functions are products of the lattice and electronic wavefunctions, and the perturbing Hamiltonian operates on the product wavefunctions. The transition rates are then proportional to the square of the matrix elements of the perturbing Hamiltonian. The applicability of this method to the alkali halides is quite questionable for very low energy electrons, as for example, those at the bottom of the conduction band, on the grounds that the coupling between the electrons and the lattice is quite strong. The electrons will always carry with them a "cloud" of polarization, i. e. , it will form a polaron. As shown by Fröhlich in his chapter in Ref. 40, one can define a dimensionless coupling parameter

$$
\alpha=e^{2}\left(\frac{1}{\epsilon_{\infty}}-\frac{1}{\epsilon}\right)\left(\frac{\mathrm{m}}{2 \omega \mathrm{h}^{3}}\right)^{1 / 2}=2\langle\mathrm{~N}\rangle
$$


where $\mathrm{e}$ is the electronic charge, $\epsilon$ is the static dielectric constant, $\epsilon_{\infty}$ is the square of the refractive index in the visible range, $m$ is the mass of the electron and $\omega$ is the angular frequency of the longitudinal optical vibrations. $\langle N\rangle$ is the average number of phonons which clothe the electron, as shown by Pines. ${ }^{40}$ This latter author shows that, in terms of $\alpha$, one can define the difference between the energy of a free electron $\mathrm{p}^{2} / 2 \mathrm{~m}$ and that of a polaron by $\Delta \mathrm{E}$ obtained from the expression

$$
\frac{\mathrm{p}^{2}}{2 \mathrm{~m}}+\Delta \mathrm{E}=-\alpha \hbar \omega+\frac{\mathrm{p}^{2}}{2 \mathrm{~m}}\left(1-\frac{\alpha}{6}\right)+\left(\mathrm{p}^{4}\right)
$$

for small p.

The first term on the right-hand side corresponds to a constant energy shift while the second term indicates that the effective mass of the polaron $\mathrm{m}^{*}$ is $\mathrm{m}^{*}=\mathrm{m} /[1-(\alpha / 6)]$.

These results are obtained by perturbation theory which is only applicable for the case in which $\Delta \mathrm{E}$ is small (i.e., small perturbation). For the alkali halides, $\alpha$ is in the neighborhood of 6 and the interaction is believed to be too strong for good quantitative use of perturbation theory. Recently the mass of the polaron has been measured by cyclotron resonance in several materials. ${ }^{41.42}$ By using a formula for $\mathrm{m}^{*} / \mathrm{m}$ related to the one above and the theoretical value for $\alpha$, the effective mass of the bare electron near the bottom of the conduction band is calculated. For $\mathrm{KCl}$ and for $\mathrm{KI}$ the band masses thus calculated are in reasonable agreement with the effective masses obtained from crystal band calculations. This seems to indicate that in spite of the above remarks perturbation theory may be applicable even to very low energy electrons in the alkali halides.

For higher energy electrons, for example, with energies much higher than the Reststrahl energy (which is of the order of $.1 \mathrm{eV}$ ), the above theory for the coupling coefficient breaks down and no theory seems to exist giving the energy dependence of $\alpha .{ }^{43}$ It is reasonable to expect a decreased coupling coefficient 
as the electrons move faster, resulting in a better validity of the solution by perturbation theory. This may explain the good quantitative results of Frohlich 33,34 and will justify the present solution of the problem by perturbation theory. This is the type of solution used by Dekker ${ }^{35,36}$ and by Khokley and van Vliet. ${ }^{38,39}$

The steps leading to the determination of the matrix elements for the electronphonon interaction are to be found in Ziman. ${ }^{44}$ However, for the sake of clarity and completeness, they will be reproduced below in more detail when it is of advantage to the reader.

The complete quantum mechanical treatment of the lattice vibrations can best be understood for a linear lattice. The extension to a three-dimensional lattice with a base (one alkali and one halide ion per lattice point, for example) will then be very easy in concept.

Consider a linear lattice of equal atoms at a distance "a". The general Hamiltonian for the system is then

$$
\mathrm{H}=\frac{1}{2} \sum_{\ell} \frac{\mathrm{p}_{\ell}^{2}}{\mathrm{~m}}+\mathrm{V}\left(\mathrm{x}_{1}, \mathrm{x}_{2}, \mathrm{x}_{3}, \ldots\right) .
$$

For interaction with nearest neighbors only,

$$
\mathrm{V}\left(\mathrm{x}_{1}, \mathrm{x}_{2}, \mathrm{x}_{3}, \ldots\right)=\sum_{\ell} \mathrm{f}\left(\mathrm{x}_{\ell+1}-\mathrm{x}_{\ell}\right)
$$

where $\mathbf{f}$ is a function only of the distance between consecutive atoms. Letting $u_{\ell}=x_{\ell}-\ell$ a be the displacement from equilibrium of the $\ell^{\text {th }}$ atom, and realizing that at the equilibrium position $u_{1}=u_{2}=0$ the derivative $\partial \mathrm{V} / \partial \mathrm{u}_{\ell}=0$, we can expand the potential

$$
\mathrm{v}\left(\mathrm{u}_{1}, \mathrm{u}_{2}, \mathrm{u}_{3}, \ldots\right)=\mathrm{v}_{0}+\frac{1}{2} \sum_{\ell^{\prime}} \mathrm{u}_{\ell^{\prime}} \mathrm{u}_{\ell^{\prime}}, \frac{\partial^{2} \mathrm{v}}{\partial \mathrm{u}_{\ell} \partial \mathrm{u}_{\ell^{\prime}}}
$$

The derivative $\partial^{2} \mathrm{v} / \partial u_{\ell} \partial u_{\ell^{\prime}}$ can be taken to be force constants "g", non-zero only for nearest neighbors. The Hamiltonian of the system can then be rewritten as

$$
\begin{aligned}
\mathrm{H}=\frac{1}{2 \mathrm{~m}} \sum_{\ell} \mathrm{p}_{\ell} \mathrm{p}_{\ell} & +\frac{1}{2} \mathrm{~g} \sum_{\ell}\left(2 \mathrm{u}_{\ell} \mathrm{u}_{\ell}-\mathrm{u}_{\ell} \mathrm{u}_{\ell+1}-\mathrm{u}_{\ell} \mathrm{u}_{\ell-1}\right) . \\
& -30-
\end{aligned}
$$


For the quantum mechanical solution, all we have to assume is that the $\mathrm{p}_{\ell}$ and $u_{\ell}$ are operators obeying the standard commutation relation for conjugate $d y-$ namical variables

$$
\left[\mathrm{u}_{\ell^{\prime}}, \mathrm{p}_{\ell^{\prime}}\right]=i \hbar \delta_{\ell \ell^{\prime}}
$$

Applying periodic boundary conditions, we know from group theory that for $\mathrm{N}$ atoms in the chain, there will be $\mathrm{N}$ representations and $\mathrm{N}$ eigenfunctions to the Hamiltonian. The representations are $\mathrm{e}^{(\mathrm{i} 2 \pi \mathrm{n} l / \mathrm{N})}, 0<\mathrm{n} \leq \mathrm{N}$. A wavefunction $|n\rangle$ will transform under a translation by $m$ lattice sites as

$$
\left|n_{+m}\right\rangle=e^{(i 2 \pi n m / N)}|n\rangle . \quad \text { Let } \frac{2 \pi n}{N}=q \text {. }
$$

In trying to find a series of wavefunctions which transform as prescribed above, we see that

$$
\mathrm{U}_{\mathrm{q}}=\frac{1}{\sqrt{\mathrm{N}}} \sum_{\ell} \mathrm{u}_{\ell} \mathrm{e}^{\mathrm{iq \ell}}, \quad 0<\ell \leq \mathrm{N}
$$

will fulfill the requirement. This is basically a Fourier series and $\mathrm{U}_{\mathrm{q}}$ is an operator formed by a linear combination of operators $u_{\ell}$. In order to place the new operators in the Hamiltonian, we have to find $u_{\ell}$ in terms of $U_{q}$. Inverse trans forming

$$
\frac{1}{\sqrt{\mathrm{N}}} \sum_{\mathrm{q}} \mathrm{U}_{\mathrm{q}} \mathrm{e}^{-\mathrm{iq} \ell}=\frac{1}{\mathrm{~N}} \sum_{\mathrm{q}} \sum_{\ell^{\prime}} \mathrm{u}_{\ell^{\prime}} \mathrm{e}^{\mathrm{iq}\left(\ell-\ell^{\prime}\right)}=\frac{1}{\mathrm{~N}} \sum_{\ell^{\prime}} u_{\ell^{\prime}} \mathrm{N} \delta_{\ell^{\prime}}=u_{\ell^{\prime}} .
$$

Similarly, the conjugate momentum operator can be taken to be

$$
\mathrm{p}_{\mathrm{q}}=\frac{1}{\sqrt{\mathrm{N}}} \sum_{\ell} \mathrm{p}_{\ell} \mathrm{e}^{-\mathrm{iq \ell}} \quad \text { or } \quad \mathrm{p}_{\ell}=\frac{1}{\sqrt{\mathrm{N}}} \sum_{\mathrm{q}} \mathrm{p}_{\mathrm{q}} \mathrm{e}^{\mathrm{iq} \ell} \text {. }
$$

The commutation relation

$$
\left[\mathrm{U}_{\mathrm{q}^{\prime}}, \mathrm{P}_{\mathrm{q}^{\prime}}\right]=i \hbar \delta \mathrm{qq}^{\prime}
$$

is obeyed. 
Substituting into the Hamiltonian and reducing, we obtain

$$
\mathrm{H}=\frac{1}{2 \mathrm{~m}} \sum_{\mathrm{q}} \mathrm{P}_{\mathrm{q}} \mathrm{P}_{-\mathrm{q}}+\mathrm{g} \sum_{\mathrm{q}} \mathrm{U}_{\mathrm{q}} \mathrm{U}_{-\mathrm{q}}(1-\cos \mathrm{q}) \text {. }
$$

The range of $q$ for the summations has been taken to be $-\pi<q \leq \pi$ (the first Brillouin zone).

Since $P_{-q}=P_{q}^{*}$, the Hamiltonian can be rewritten as a sum of independent Hamiltonians

$$
\mathrm{H}=\sum\left(\frac{1}{2 \mathrm{~m}} \mathrm{P}_{\mathrm{q}} \mathrm{P}_{\mathrm{q}}^{*}+(1-\cos \mathrm{q}) \mathrm{U}_{\mathrm{q}} \mathrm{U}_{\mathrm{q}}^{*}\right)
$$

Each term is the Hamiltonian of a harmonic oscillator $H=\frac{1}{2}\left(\frac{p^{2}}{2 m}+\mathrm{kx}^{2}\right)$, where $\mathrm{k}$ corresponds to $2 \mathrm{~g}(1-\cos q)$. Since $\omega=\sqrt{\mathrm{k} / \mathrm{m}}$, we see that each independent oscillator has a frequency $\omega_{q}=\sqrt{(2 \mathrm{~g} / \mathrm{m})(1-\cos q)}=2 \sqrt{\mathrm{g} / \mathrm{m}}\left|\sin \frac{1}{2} q\right|$. The result is the well-known dispersion relation for a linear chain of atoms.

One can now form creation and annihilation operators, $a_{q}^{*}$ and $a_{q}$, respectively.

$$
\begin{aligned}
& a_{q}=\sqrt{\frac{1}{2 m \hbar \omega_{q}}} P_{q}^{*}+i \sqrt{\frac{g(1-\cos q)}{\hbar \omega_{q}}} U_{q}^{*} . \\
& a_{q}^{*}=\sqrt{\frac{1}{2 m \hbar \omega_{q}}} P_{q}^{*}+i \sqrt{\frac{g(1-\cos q)}{\hbar \omega_{q}}} U_{q} .
\end{aligned}
$$

The commutation relation is $\left[\begin{array}{cc}a_{q}, & a_{q}^{*}\end{array}\right]=1$ and the Hamiltonian becomes

$$
H=\frac{1}{2} \sum_{q} \hbar \omega_{q}\left(a_{q}^{*} a_{q}+a_{q} a_{q}^{*}\right) \text {. }
$$

The eigenstates of the annihilation and creation operators are characterized by sets of positive integers giving the number of quanta of energy $\hbar \omega_{q}$ present in a particular mode $q$. If the $q^{\text {th }}$ mode is excited to its $n^{\text {th }}$ level, we say that there are $n_{q}$ quanta in the mode, and symbolize the state by $\left|n_{q}\right\rangle$. 
The application of $a_{q}^{*}$ and $\underset{q}{a}$ operators to a state $\left|\mathrm{n}_{\mathrm{q}}\right\rangle$ yields

$$
\begin{aligned}
& a_{q}^{*}\left|n_{q}\right\rangle=\sqrt{n_{q}+1}\left|n_{q}+1\right\rangle \\
& a_{q}\left|n_{q}\right\rangle=\sqrt{n_{q}}\left|n_{q}-1\right\rangle .
\end{aligned}
$$

Applying this rule to each term in the Hamiltonian one finds that the energy of a state $\left|n_{q}\right\rangle$ is $\left(n_{q}+\frac{1}{2}\right) \hbar \omega_{q}$, and it follows that the total system energy, for a wavefunction $\left|n_{1}, n_{2}, n_{3}, \ldots\right\rangle=\left|n_{1}\right\rangle\left|n_{2}\right\rangle\left|n_{3}\right\rangle \ldots$ is

$$
\sum\left(n_{q}+\frac{1}{2}\right) \hbar \omega_{q}
$$

For three-dimensional lattices with a base, the procedure closely follows the one given above, with substantially more complexity. The development will not be given here; Ziman ${ }^{44}$ covers it in detail. However, the results presented below follow very intuitively from the linear lattice case presented above.

For a three-dimensional lattices with one atom per unit cell it is found that for each $\vec{q}$ (which is now a vector within the first Brillouin zone) there are three normal modes of vibration. The physical meaning of these three modes is easy to see if one chooses $\vec{q}$ in the direction of one of the three orthogonal coordinates. Then one mode can be termed longitudinal and the other two transverse, for atomic displacements in the direction of $\vec{q}$, or perpendicular to it, respectively. If the base contains more than one atom, one new branch with three normal modes appears in the dispersion relations for each added atom. For the alkali halide one obtains, then, the well-known acoustical and optical branches of lattice vibrations.

The Hamiltonian for a given wavevector $\vec{q}$ is then

$$
\mathrm{H}_{\overrightarrow{\mathrm{q}}}=\frac{1}{2} \sum_{\mathrm{p}}\left(\mathrm{U}_{\overrightarrow{\mathrm{q}}, \mathrm{p}} \mathrm{U}_{\overrightarrow{\mathrm{q}}, \mathrm{p}}^{*}+\omega_{\overrightarrow{\mathrm{q}}, \mathrm{p}}^{2} \mathrm{P}_{\overrightarrow{\mathrm{q}}, \mathrm{p}} \mathrm{P}_{\overrightarrow{\mathrm{q}}, \mathrm{p}}^{*}\right)
$$

where $U$ and $P$ are conjugate normal coordinate operators and $\mathrm{p}$ is an index indicating the particular branch and mode. One can also define creation and 
annihilation operators obeying the commutation relation

$$
\left[a_{q, p}, a_{q^{\prime}, p^{\prime}}^{*}\right]=\delta_{q q^{\prime}} \delta_{p p^{\prime}}
$$

For a given branch and mode, we drop the subscript $\mathrm{p}$ and the Hamiltonian becomes

$$
H=\frac{1}{2} \sum_{\vec{q}} \hbar \omega_{\vec{q}}\left(a_{\vec{q}}^{*} a_{\vec{q}}+a_{\vec{q}} a_{\vec{q}}^{*}\right)
$$

which is in a convenient form for subsequent use. The energy for a particular mode and branch for an oscillator with wavenumber $\vec{q}$ is

$$
\left(\mathrm{n}_{\overrightarrow{\mathrm{q}}}+\frac{1}{2}\right) \hbar \omega_{\overrightarrow{\mathrm{q}}}
$$

The unperturbed electronic wavefunctions have been taken ${ }^{33,34}$ to be planewaves $|\mathrm{k}\rangle=\left(1 / \mathrm{V}^{1 / 2}\right) \mathrm{e}^{\overrightarrow{\mathrm{ik}} \cdot \overrightarrow{\mathrm{r}}}$ with energy $E_{\overrightarrow{\mathrm{k}}}=\hbar^{2} \mathrm{k}^{2} / 2 \mathrm{~m}^{*}$ where $\mathrm{m}^{*}$ is the effective mass of the electron. For the problem of secondary emission it seems necessary to assume that the secondaries move about the crystal with some single effective mass $\mathrm{m}^{*}$ for all the range of energies that the electrons will have between generation and escape. This can span as much as $1.5 \mathrm{eV}$ for electrons in materials with large energy losses, as will be shown below. The assumption can only be strictly true for free electrons, in which case $\mathrm{m}^{*}=\mathrm{m}$. If the bands are not very different from free electron bands, then most of the electrons still would have $m^{*}=m$. A few calculations of band structure of alkali halides already exist. Oyama and Miyakawa ${ }^{45}$ have made calculations on $\mathrm{KCl}$, and Onadera, Okazaki and Inui $^{46}$ have calculated those of KI. The bands have a general resemblance to free electron bands but they are quite different in detail. Effective masses have been calculated for electrons near the bottom of the conduction band and found to be $.4 \mathrm{~m}$ for $\mathrm{KCl}$ and $.49 \mathrm{~m}$ for $\mathrm{KI}$. These numbers have little relationship, however, with an overall effective mass.

There are possibly two arguments which support the treatment of conduction electrons as almost free electrons in the alkali halides. One is given by J. C. Phillips ${ }^{47}$ 
in which he points out that conduction electrons see only monovalent ions plus closed shells and on chemical grounds one expects the effect of the closed shells to be very small. The effect of the monovalent ions is to give band splittings of the order of $1 \mathrm{eV}$. The second argument is based on observations made by T. DiStephano ${ }^{48}$ on the energy distribution of electrons photo-emitted from evaporated films of CsI. By changing the energy of the bombarding UV photons, he finds no changes in the shape of the spectrum of photo-emitted electrons, except for some minor oscillations. This is interpreted as indicating that there is no important structure in the conduction band of CsI, i. e., that the bands are very nearly free electron-like. This point, however, needs more studying, as Krolikowski 49 has found substantial structure in the conduction bands of $\mathrm{CsBr}, \mathrm{CsCl}, \mathrm{CsI}$ and $\mathrm{KI}$. Another difficulty already mentioned is that of the effective mass of the polaron, which should result in an increased $\mathrm{m}^{*}$. With the assumption of low coupling, though, this increase in mass may be quite small.

One can conclude, therefore, that it may be moderately reasonable to represent the secondary electrons as nearly free electrons. The overall effective mass, $\mathrm{m}^{*}$, will be an adjustable parameter in the calculations, but it is not expected to deviate much from the free electronic mass.

Having thus described the eigenstates of the unperturbed system, we turn our attention to the interaction Hamiltonian, following Ziman again, ${ }^{44}$ and adding information from Fröhlich. ${ }^{50}$ Considering the lattice to be a continuum, one can define a polarization $\overrightarrow{\mathrm{P}}(\overrightarrow{\mathrm{r}})$ per unit volume, which will have two parts, (1) $\overrightarrow{\mathrm{P}}_{0}(\overrightarrow{\mathrm{r}})$ due to the deformation of the electron shells of the ions under the influence of an applied field, and (2) $\vec{P}_{\text {ir }}(\vec{r})$ which is due to the relative displacement of the ions in the crystal. The first part of $\overrightarrow{\mathrm{P}}_{0}(\overrightarrow{\mathrm{r}})$ has a value which is practically independent of frequency until one gets to the ultraviolet absorption region. As long as one is discussing interactions with electrons of energy substantially below that of the 
UV absorption edge, it has been customary to neglect $\vec{P}_{0}(\vec{r})$ completely. The second part is due to the optical modes of lattice vibrations, and when driven by an external field with electric displacement $\vec{D}(\vec{r})$ it will satisfy a dynamic equation of the form

$$
\ddot{\vec{P}}_{\mathrm{ir}}(\overrightarrow{\mathrm{r}})+\omega^{2} \overrightarrow{\mathrm{P}}_{\mathrm{ir}}(\overrightarrow{\mathrm{r}})=\frac{1}{\gamma} \overrightarrow{\mathrm{D}}(\overrightarrow{\mathrm{r}})
$$

where $\gamma$ is a constant separating the part of the dielectric constant which is due to lattice polalization, given by

$$
\frac{1}{\gamma}=\frac{\omega^{2}}{4 \pi}\left(\frac{1}{\epsilon_{\infty}}-\frac{1}{\epsilon}\right)
$$

The frequency $\omega$ is related to the observable residual ray or Reststrahlen absorption frequency $\omega_{\mathrm{r}}$ by $^{51}$

$$
\omega=\left(\frac{\epsilon}{\epsilon_{\infty}}\right)^{1 / 2} \omega_{r} .
$$

The theoretical dispersion relations for $\omega$ vs. $\vec{q}$ show quite flat bands for the optical branch of lattice vibrations and it is found experimentally that $\omega_{r}$ is well-defined by absorption line in the alkali halides. This allows the use of a single $\omega_{\mathrm{r}}$ and $\omega$ for all $\overrightarrow{\mathrm{q}}$, resulting in a substantial simplication of the problem.

The above equation of motion could be obtained from a total Hamiltonian

$$
\mathrm{H}=\int\left\{\frac{1}{2} \gamma\left(\dot{\overrightarrow{\mathrm{P}}}_{\mathrm{ir}}^{2}+\omega^{2} \overrightarrow{\mathrm{P}}_{\mathrm{ir}}^{2}\right)-\overrightarrow{\mathrm{D}} \cdot \overrightarrow{\mathrm{P}}_{\mathrm{ir}}\right\} \mathrm{dr}
$$

of which the part between parenthesis corresponds to the energy of the harmonic oscillators and the energy of interaction is

$$
\mathrm{H}_{\text {inter }}=\int \overrightarrow{\mathrm{D}} \cdot \overrightarrow{\mathrm{P}}_{\mathrm{ir}} \mathrm{d \vec { \textrm {r } }}
$$


$\overrightarrow{\mathrm{P}}_{\text {ir }}$ and $\gamma \dot{\overrightarrow{\mathrm{P}}}_{\mathrm{ir}}$ are conjugate coordinates in the Hamiltonian. It is now useful to introduce a complex field vector $\vec{B}(\vec{r})$ such that

$$
\begin{aligned}
& \overrightarrow{\mathrm{B}}(\overrightarrow{\mathrm{r}})=\left(\frac{\gamma \omega}{2 \mathrm{~h}}\right)^{1 / 2}\left(\overrightarrow{\mathrm{P}}_{\mathrm{ir}}(\overrightarrow{\mathrm{r}})+\frac{\mathrm{i}}{\omega} \dot{\overrightarrow{\mathrm{P}}}_{\mathrm{ir}}(\overrightarrow{\mathrm{r}})\right) \\
& \overrightarrow{\mathrm{B}}^{*}(\overrightarrow{\mathrm{r}})=\left(\frac{\gamma \omega}{2 \mathrm{~h}}\right)^{1 / 2}\left(\overrightarrow{\mathrm{P}}_{\mathrm{ir}}(\overrightarrow{\mathrm{r}})-\frac{\mathrm{i}}{\omega} \dot{\overrightarrow{\mathrm{P}}}_{\mathrm{ir}}(\overrightarrow{\mathrm{r}})\right)
\end{aligned}
$$

such that vectors $\overrightarrow{\mathrm{P}}$ and $\dot{\overrightarrow{\mathrm{P}}}$ become

$$
\begin{aligned}
& \overrightarrow{\mathrm{P}}_{\mathrm{ir}}(\overrightarrow{\mathrm{r}})=\left(\frac{\hbar}{2 \gamma \omega}\right)^{1 / 2}\left(\overrightarrow{\mathrm{B}}^{*}(\overrightarrow{\mathrm{r}})+\overrightarrow{\mathrm{B}}(\overrightarrow{\mathrm{r}})\right) \\
& \dot{\overrightarrow{\mathrm{P}}}_{\mathrm{ir}}(\overrightarrow{\mathrm{r}})=\left(\frac{\hbar \omega}{2 \gamma}\right)^{1 / 2} \mathrm{i}\left(\overrightarrow{\mathrm{B}^{*}}(\overrightarrow{\mathrm{r}})-\overrightarrow{\mathrm{B}}(\overrightarrow{\mathrm{r}})\right)
\end{aligned}
$$

with which the Hamiltonian becomes

$$
H=\hbar \omega \int \vec{B}^{*}(\vec{r}) \cdot \vec{B}(\vec{r}) d^{3}{ }^{3} \frac{\hbar}{2 \gamma \omega}^{1 / 2} \int \vec{D}(\vec{r}) \cdot\left[\vec{B}^{*}(\vec{r})+\vec{B}(\vec{r})\right] d^{3} \vec{r}=H_{o}+H_{\text {inter }} .
$$

If we now consider $\vec{B}$ to be subjected to periodic boundary conditions in a crystal of volume $\mathrm{V}$ and expand in a Fourier series with terms corresponding to reciprocal lattice wave-numbers $\vec{q}$,

$$
\vec{B}(\vec{r})=\frac{1}{v^{1 / 2}} \sum_{\vec{q}} \frac{\vec{q}}{q} b_{\vec{q}} e^{i \vec{q} \cdot \vec{r}}, \quad \vec{B}^{*}(\vec{r})=\frac{1}{v^{1 / 2}} \sum_{\vec{q}} \frac{\vec{q}}{q} b_{\vec{q}}^{*} e^{-i \vec{q} \cdot \vec{r}}
$$

everything is now set for a quantization scheme. Considering $\vec{B}$ and $\vec{B}^{*}$ as operators, we examine first the non-interaction part of the Hamiltonian:

$$
\begin{aligned}
& \mathrm{H}_{0}=\frac{1}{2} \hbar \omega \int \overrightarrow{\mathrm{B}}^{*} \cdot \overrightarrow{\mathrm{B}}+\overrightarrow{\mathrm{B}} \cdot \overrightarrow{\mathrm{B}}^{*}=\frac{1}{2} \hbar \omega \sum_{\overrightarrow{\mathrm{q}}} \frac{\overrightarrow{\mathrm{q}}}{\overrightarrow{\mathrm{q}}^{\prime}} \frac{\overrightarrow{\mathrm{q}}}{\mathrm{q}^{\prime}} \mathrm{b}_{\overrightarrow{\mathrm{q}}}^{*} \mathrm{~b}_{\overrightarrow{\mathrm{q}}^{\prime}} \frac{1}{\mathrm{~V}} \int \mathrm{d} \overrightarrow{\mathrm{r}}^{3} \mathrm{e}^{\mathrm{i}(\overrightarrow{\mathrm{q}}-\overrightarrow{\mathrm{q}}) \cdot \overrightarrow{\mathrm{r}}}+\text { Herm. conj} \text {. } \\
& =\frac{1}{2} \hbar \omega \sum_{\overrightarrow{\mathrm{q}} \overrightarrow{\mathrm{q}}} \frac{\overrightarrow{\mathrm{q}}}{\mathrm{q}} \cdot \frac{\overrightarrow{\mathrm{q}}^{*}}{\overline{\mathrm{q}}} \mathrm{b}_{\overrightarrow{\mathrm{q}}}^{*} \mathrm{~b}_{\overrightarrow{\mathrm{q}}}, \delta_{\overrightarrow{\mathrm{q}} \overrightarrow{\mathrm{q}}^{\prime}}+\text { Herm. Conj. }=\frac{1}{2} \hbar \omega \sum_{\overrightarrow{\mathrm{q}}}\left(\mathrm{b}_{\overrightarrow{\mathrm{q}}}^{*} \mathrm{~b}_{\overrightarrow{\mathrm{q}}}+\mathrm{b}_{\overrightarrow{\mathrm{q}}} \mathrm{b}_{\overrightarrow{\mathrm{q}}}^{*}\right) .
\end{aligned}
$$

Comparison of Eqs. (6) and (4) shows that the operators defined here as $\mathrm{b}_{\mathrm{q}}^{*}$ and $b_{q}$ are identical to the phonon creation and annihilation operators $a_{\vec{q}}^{*}$ and $a_{\vec{q}}$. 
It is easiest to find $\mathrm{H}_{\text {inter }}$ by considering the potential seen by an electron at $\mathrm{r}$ due to the polarization of the lattice.

$$
4 \pi \overrightarrow{\mathrm{P}}_{\text {ir }}=\nabla \phi
$$

and

$$
\mathrm{H}_{\text {inter }}=\mathrm{e} \phi(\overrightarrow{\mathrm{r}})
$$

Substituting for $\overrightarrow{\mathrm{P}}_{\text {ir }}$ in terms of the operators $\mathrm{a}_{\overrightarrow{\mathrm{q}}}^{*}$ and $\mathrm{a}_{\overrightarrow{\mathrm{q}}}$ into Eq. (7) and solving for $\phi(\vec{r})$, we obtain

$$
H_{\text {inter }}=4 \pi i\left(\frac{e^{2} \hbar}{2 \gamma \omega V}\right)^{1 / 2} \sum_{\vec{q}} \frac{1}{q}\left(a_{\vec{q}}^{*} e^{-i \vec{q} \cdot \vec{r}}-a_{q} e^{i \vec{q} \cdot \vec{r}}\right), \quad q \neq 0 .
$$

The limitation of $q \neq 0$ is discussed by Fröhlich. ${ }^{49}$ The $\vec{q} \neq 0$ term corre sponds to a d.c. polarization which cannot exist by the requirement of charge neutrality.

The matrix element for the transition of one electron in state $\vec{k}$ to $\vec{k}$ with the loss of one phonon of wavenumber $\vec{q}$ can now be obtained:

$$
M^{-}\left(\vec{k}, \vec{k}^{\prime}\right)=\left\langle\vec{k}, n_{\vec{q}}\left|H_{\text {inter }}\right| \overrightarrow{k^{\prime}}, n_{\vec{q}}-1\right\rangle
$$

Since

$$
\begin{aligned}
& \left\langle\mathrm{n}_{\overrightarrow{\mathrm{q}}}\left|\mathrm{a}_{\overrightarrow{\mathrm{q}}}\right| \mathrm{n}_{\overrightarrow{\mathrm{q}}}-1\right\rangle=0 \quad \text { and }\left\langle\mathrm{n}_{\overrightarrow{\mathrm{q}}}\left|\mathrm{a}_{\overrightarrow{\mathrm{q}}}\right| \mathrm{n}_{\overrightarrow{\mathrm{q}}}-1\right\rangle=\sqrt{\mathrm{n}_{\overrightarrow{\mathrm{q}}}} \\
& \mathrm{M}^{-}\left(\mathrm{k}, \mathrm{k}^{\prime}\right)=4 \pi \mathrm{i}\left(\frac{\mathrm{e}^{2} \hbar}{2 \gamma \omega V}\right)^{1 / 2} \sum_{\overrightarrow{\mathrm{q}}} \frac{1}{\mathrm{q}} \sqrt{\mathrm{n}_{\overrightarrow{\mathrm{q}}}} \frac{1}{\mathrm{~V}} \int \mathrm{d}^{3} \mathrm{e}^{-\mathrm{ik} \cdot \overrightarrow{\mathrm{r}}} \mathrm{e}^{-\mathrm{i} \overrightarrow{\mathrm{q}} \cdot \overrightarrow{\mathrm{r}}} \mathrm{e}^{\mathrm{i \vec {k } \cdot \vec { r }}}
\end{aligned}
$$

or

$$
M^{-}\left(\vec{k}, \vec{k}^{\prime}\right)=4 \pi i\left(\frac{e^{2} \hbar}{2 \gamma \omega V}\right)^{1 / 2} \frac{1}{q} \sqrt{n_{\vec{q}}} \text { for } \overrightarrow{k^{\prime}}=\vec{k}+\vec{q} \text {. }
$$


Similarly, for transitions with the gain of one phonon, one obtains

$$
\mathrm{M}^{+}\left(\overrightarrow{\mathrm{k}}, \overrightarrow{\mathrm{k}^{\prime}}\right)=-4 \pi \mathrm{i}\left(\frac{\mathrm{e}^{2} \hbar}{2 \gamma \omega \mathrm{V}}\right)^{1 / 2} \frac{1}{\mathrm{q}} \sqrt{\mathrm{n}_{\overrightarrow{\mathrm{q}}}+1} \text { for } \overrightarrow{\mathrm{k}}=\overrightarrow{\mathrm{k}^{\prime}}+\overrightarrow{\mathrm{q}}
$$

For the problem under study, $\mathrm{n}_{\overrightarrow{\mathrm{q}}}$ is a constant depending only on the material and temperature. The vector on the subscript will be dropped.

\section{Rate and Angle of Scattering}

The transition rate for electrons from a state $|k\rangle$ to a state $\left|k^{\prime}\right\rangle$ is given by the standard result of perturbation theory

$$
\mathrm{W}_{\overrightarrow{\mathrm{k}} \overrightarrow{\mathrm{k}}^{\prime}}=\frac{1}{\hbar^{2}}\left|\mathrm{H}_{\overrightarrow{\mathrm{k}} \overrightarrow{\mathrm{k}}^{\prime}}^{\prime}\right|^{2} \frac{\partial}{\partial t} \frac{\sin ^{2} \beta^{t}}{\beta^{2}} \quad \text { where } \beta=\frac{1}{2 \hbar}\left[\varepsilon_{\overrightarrow{\mathrm{k}}},-\left(\tilde{\sigma}_{\overrightarrow{\mathrm{k}}}+\hbar \omega\right)\right]
$$

for the case of an upward transition in energy. $\mathrm{H}_{\mathrm{k}}^{\prime}{ }^{\prime}$ is the matrix element of the interaction.

For the case of phonon annihilation, the rate will be

$$
\mathrm{W}_{\overrightarrow{\mathrm{k}} \overrightarrow{\mathrm{k}^{\prime}}}=\frac{1}{\hbar^{2}} \frac{(4 \pi)^{2} e^{2} \hbar}{2 \gamma \omega \mathrm{V}} \frac{1}{\mathrm{q}^{2}} \mathrm{n}_{\mathrm{q}} \frac{\partial}{\partial \mathrm{t}} \frac{\sin ^{2} \beta \mathrm{t}}{\beta^{2}} \text {. }
$$

It will be convenient to write $\beta$ in terms of $\mathrm{k}$ and $\mathrm{k}^{\prime}$. Since $\varepsilon_{\mathrm{k}}=\frac{\hbar^{2} \mathrm{k}^{2}}{2 \mathrm{~m}^{*}}$ and $\sigma_{\mathrm{k}^{\prime}}=\frac{\hbar^{2} \mathrm{k}^{2}}{2 \mathrm{~m}^{*}}$

$$
\beta=\frac{\hbar}{4 \mathrm{~m}^{*}} \mathrm{k}^{\prime 2}-\left(\frac{\hbar}{4 \mathrm{~m}^{*}} \mathrm{k}^{2}+\frac{\omega}{2}\right)
$$

The total scattering rate from a state $\vec{k}$ to all the other available states $\vec{k}^{\prime}$ can now be found by summing over all $\vec{k}^{\prime}$, but it is more convenient to do the sum as an integral over $\vec{q}$ as follows:

Figure 6a shows the angular relationship between $\vec{k}, \overrightarrow{k^{\prime}}$ and $\vec{q}$ in wavenumber space. In particular, $\mathrm{q}^{2}=\mathrm{k}^{2}+\mathrm{k}^{2}-2 \mathrm{kk}^{\prime} \cos \theta$, and also $\mathrm{k}^{\mathbf{\prime}^{2}}=\mathrm{k}^{2}+\mathrm{q}^{2}-2 \mathrm{kq} \cos \theta^{*}$. Also in a crystal of volume $\mathrm{V}$, the number of longitudinal modes per unit volume in $q$ space is $V / 8 \pi^{3}$ so that a sum 


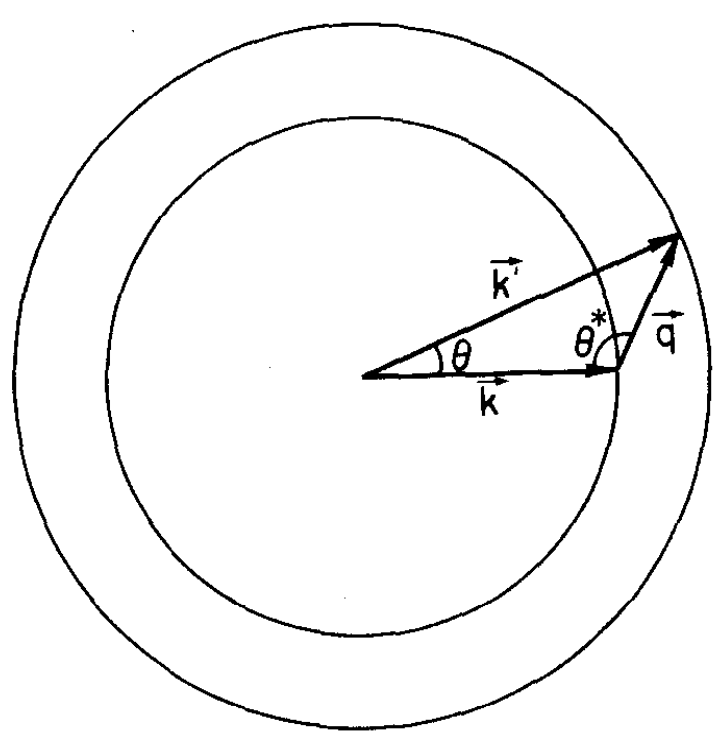

(a)

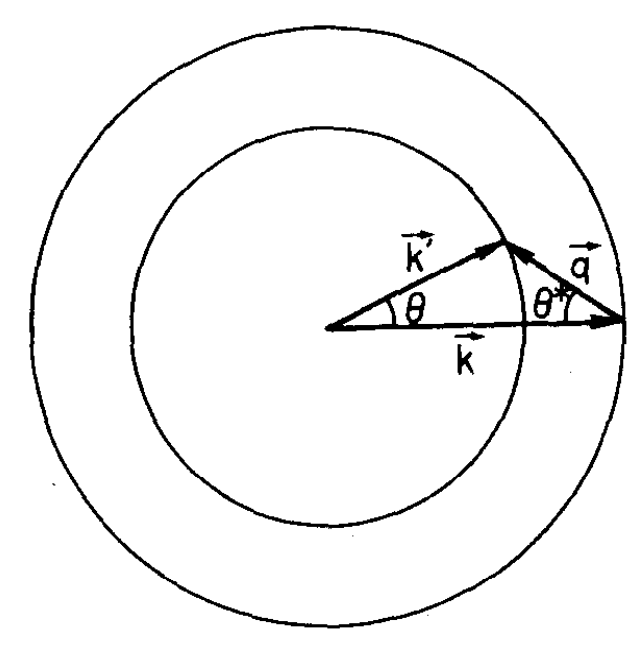

(b) $\overline{987 A 6}$

Fig. 6--Relationship between vectors $\vec{q}, \vec{k}$, and $\overrightarrow{k^{\prime}}$ in wave-vector space (a) For phonon annihilation interaction

(b) For phonon creation interaction 
over all q becomes

$$
\frac{\mathrm{V}}{8 \pi^{3}} \iiint \mathrm{d}_{\mathrm{q}}^{3} .
$$

Since the element of volume $d_{q}^{3}$ is $q^{2} d q \sin \theta^{*} d \theta^{*} d \phi$, we have

$$
\mathrm{w}_{\overrightarrow{\mathrm{k}}}=\int_{\mathrm{q}=\mathrm{q}_{\min }}^{\mathrm{q} \max } \int_{\phi=0}^{2 \pi} \int_{\theta^{*}=0}^{\pi} \frac{1}{\hbar^{2}} \frac{(4 \pi)^{2} \mathrm{e}^{2} \hbar}{2 \gamma \omega \mathrm{V}} \frac{1}{\mathrm{q}^{2}} \mathrm{n}_{\mathrm{q}} \frac{\partial}{\partial \mathrm{t}} \frac{\sin ^{2} \beta \mathrm{t}}{\beta^{2}} \frac{\mathrm{V}}{8 \pi^{2}} \mathrm{q}^{2} \mathrm{dq} \sin \theta^{*} \mathrm{~d} \theta^{*} \mathrm{~d} \phi
$$

or

$$
\mathrm{W}_{\overrightarrow{\mathrm{k}}}^{-}=\int_{\mathrm{q}_{\min }}^{\mathrm{q}_{\max }} \int_{0}^{\pi} \frac{2 \mathrm{e}^{2} \mathrm{n} q}{\not i \gamma \omega} \frac{\partial}{\partial \mathrm{t}} \frac{\sin ^{2} \beta \mathrm{t}}{\beta^{2}} \mathrm{dq} \sin \theta^{*} \mathrm{~d} \theta^{*} .
$$

In terms of $q, \mathrm{k}$ and $\theta^{*}$,

$$
\beta=\frac{\hbar}{4 m^{*}} q^{2}-\frac{\not K}{2 m^{*}} k q \cos \theta^{*}-\frac{\omega}{2}
$$

and

$$
\mathrm{d} \beta=\frac{\hbar \mathrm{K}}{2 \mathrm{~m}} \mathrm{kq} \sin \theta^{*} \mathrm{~d} \theta^{*},
$$

so that we can rewrite

$$
\mathrm{W}_{\overrightarrow{\mathrm{k}}}^{-}=\int_{\mathrm{q}_{\min }}^{\mathrm{q}_{\max }} \int_{\left(\frac{\hbar}{4 \mathrm{~m}^{*}} \mathrm{q}^{2}-\frac{\hbar}{2 \mathrm{~m}^{*}} \mathrm{kq}-\frac{\omega}{2}\right)}^{\left(\frac{\hbar^{2}}{4 \mathrm{~m}^{*}} \mathrm{q}^{2}+\frac{\hbar}{2 \mathrm{~m}^{*}} \mathrm{kq}-\frac{\omega}{2}\right)} \frac{4 \mathrm{e}^{2} \mathrm{~m}^{*} \mathrm{n} \mathrm{q}}{\hbar^{2} \gamma \omega} \frac{1}{\mathrm{kq}} \frac{\partial}{\partial \mathrm{t}} \frac{\sin ^{2} \beta \mathrm{t}}{\beta^{2}} \mathrm{~d} \beta \mathrm{dq} .
$$

Since the interval of integration for $\beta$ covers the point $\beta=0$, except for $\theta^{*}=\pi$, or $\theta^{*}=0$ (which is of little importance), we can approximate the integral for constant $q$ over $\beta$ to be $\pi t$, obtaining also the conservation of energy requirement, i.e., the only significant contribution to the integral is obtained when $\beta \approx 0$. 
We are then left with

$$
\mathrm{W}_{\overline{\mathrm{k}}}^{-}=\int_{\mathrm{q}_{\min }}^{\mathrm{q}_{\max }} \frac{4 \pi \mathrm{e}^{2} \mathrm{~m}^{*} \mathrm{n}_{\mathrm{q}}}{\hbar^{2} \gamma \omega} \frac{1}{\mathrm{k}} \frac{1}{\mathrm{q}} \mathrm{dq}
$$

In order to find now the total transition rate we have to integrate over all possible q. The limits of integration are set by the conservation of energy to insure the existence of a $\theta^{*}$ which satisfies $\beta=0$ ( Ziman, ${ }^{44}$ sect. 10.5).

From Eq. (10), setting $\beta=0$ and solving for $q$, we obtain

$$
q=\cos \theta^{*} \mathrm{k} \pm \sqrt{\cos ^{2} \theta^{*} \mathrm{k}^{2}+\frac{2 \mathrm{~m}^{*} \omega}{\hbar}} .
$$

Allowing now $\cos \theta^{*}$ to range from -1 to 1 , we obtain the minimum and maximum positive values of $q$ possible

$$
\mathrm{k}\left[\left(1+\frac{\hbar \omega}{\varepsilon_{\mathrm{k}}}\right)^{1 / 2}-1\right]<\mathrm{q}<\mathrm{k}\left[\left(1+\frac{\hbar \omega}{\delta_{\overrightarrow{\mathrm{k}}}}\right)^{1 / 2}+1\right]
$$

Integrating Eq. (11) over these limits we obtain

$$
\mathrm{W}_{\overrightarrow{\mathrm{k}}}=\frac{4 \pi \mathrm{e}^{2} \mathrm{~m}^{*} \mathrm{n}_{\mathrm{q}}}{\hbar^{2} \gamma \omega} \frac{1}{\mathrm{k}} \ln \left\{\frac{\left(1+\frac{\hbar \omega}{\mathrm{g} \overrightarrow{\mathrm{k}}}\right)^{1 / 2}+1}{\left(1+\frac{\hbar \omega}{\delta \overrightarrow{\mathrm{k}}}\right)^{1 / 2}-1}\right\}
$$

For transitions between electronic states $|\vec{k}\rangle$ and $|\vec{k}\rangle$ with the creation of one phonon of wavevector $\vec{q}$, Fig $6 \mathrm{~b}$ shows the angular relationships of interest. For this case

$$
\begin{aligned}
& \beta=\left(\frac{\hbar}{4 m^{*}} \mathrm{k}^{2}+\frac{\omega}{2}\right)-\frac{\not k}{4 \mathrm{~m}^{*}} \mathrm{k}^{2}=\frac{\not}{4 \mathrm{~m}^{*}} \mathrm{q}^{2}-\frac{\hbar}{2 \mathrm{~m}^{*}} \mathrm{kq} \cos \theta^{*}+\frac{\omega}{2} \\
& \mathrm{~W}_{\mathrm{k}}^{+}=\int_{\mathrm{q}_{\min }}^{\mathrm{q}_{\max }} \frac{4 \pi \mathrm{e}^{2} \mathrm{~m}^{*}\left(\mathrm{n}_{\mathrm{q}}+1\right)}{\hbar^{2} \gamma \omega} \frac{1}{\mathrm{k}} \frac{1}{\mathrm{q}} \mathrm{dq} .
\end{aligned}
$$


The limits of integration are somewhat different:

$$
\mathrm{k}\left[1-\left(1-\frac{\hbar \omega}{\overrightarrow{\mathrm{g}} \overrightarrow{\mathrm{k}}}\right)^{1 / 2}\right]<\mathrm{q}<\mathrm{k}\left[1+\left(1-\frac{\hbar \omega}{\mathrm{k}}\right)^{1 / 2}\right]
$$

leading to a total scattering rate

$$
\mathrm{W}_{\mathrm{k}}^{+}=\frac{4 \pi \mathrm{e}^{2} \mathrm{~m}^{*}\left(\mathrm{n}_{\mathrm{q}}+1\right)}{\hbar^{2} \gamma \omega} \frac{1}{\mathrm{k}} \ln \left\{\frac{1+\left(1-\frac{\hbar \omega}{\hbar \overrightarrow{\mathrm{k}}}\right)^{1 / 2}}{1-\left(1-\frac{\hbar \omega}{\mathrm{k}}\right)^{1 / 2}}\right\}
$$

Figure 7 shows a plot of $\mathrm{W}_{\mathrm{k}}^{-}$and $\mathrm{W}_{\mathrm{k}}^{+}$computed for $\mathrm{CsI}$ and $\mathrm{KCl}$ starting from the basic data published by $\mathrm{Bak}^{52}$ (given in Table 5 for convenience), for electron energies between .25 and $6 \mathrm{eV}$. An effective mass $\mathrm{m}^{*} / \mathrm{m}=1$ has been assumed. $\lambda$ is the wavelength of the longitudinal waves obtained from the residual ray absorption. Temperature is $300^{\circ} \mathrm{K}$.

The logarithmic terms in Eqs. (12) and (14) are practically identical to each other for a given $\stackrel{2}{*}$ The increase in their magnitude from $\mathscr{L}=.25 \mathrm{eV}$ to $\therefore=6 \mathrm{eV}$ is by approximately a factor of 2 , in a logarithmic fashion. 


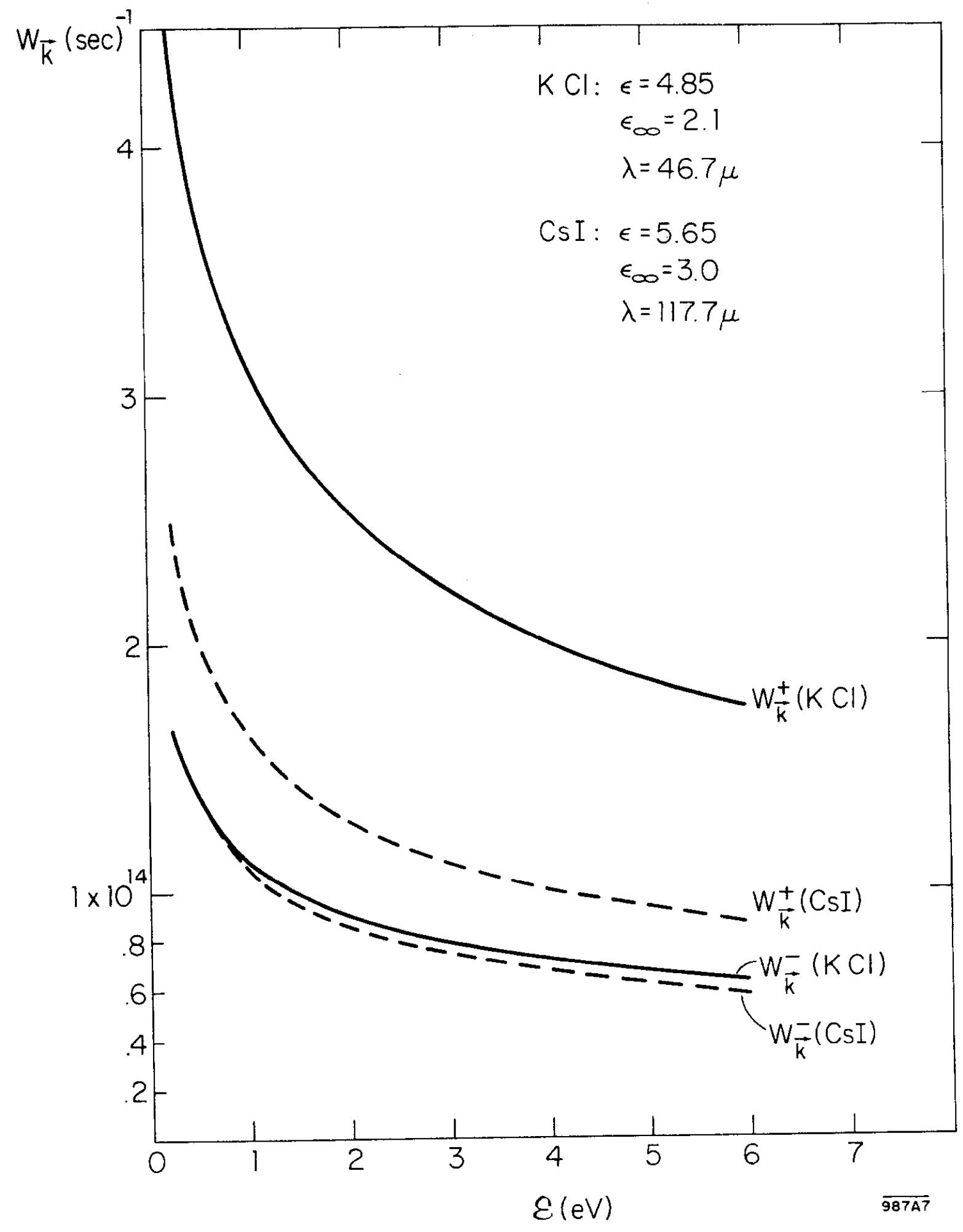

FIG. $7--\mathrm{W}_{\overrightarrow{\mathrm{k}}}^{-}$and $\mathrm{W}_{\overrightarrow{\mathrm{k}}}^{+}$, the total scattering rates as a function of electron energy, for $\mathrm{CsI}$ and $\mathrm{KCl}$ 
TABLE 5

\begin{tabular}{|c|c|c|c|}
\hline Crystal & $\epsilon$ & $\epsilon_{\infty}$ & $\begin{array}{c}\lambda \text { (microns) } \\
\text { of longitudinal waves }\end{array}$ \\
\hline $\mathrm{LiH}$ & 12.9 & 3.6 & 8.94 \\
\hline LiF & 8.9 & 1.9 & 15.1 \\
\hline $\mathrm{LiCl}$ & 12.0 & 2.7 & 25.15 \\
\hline $\mathrm{LiBr}$ & 13.2 & 3.2 & 30.8 \\
\hline $\mathrm{NaF}$ & 5.1 & 1.7 & 24.2 \\
\hline $\mathrm{NaCl}$ & 5.9 & 2.25 & 37.9 \\
\hline $\mathrm{NaBr}$ & 6.4 & 2.6 & 47.8 \\
\hline $\mathrm{KF}$ & 5.5 & 1.5 & 30.7 \\
\hline $\mathrm{KCl}$ & 4.85 & 2.1 & 46.7 \\
\hline $\mathrm{KBr}$ & 4.9 & 2.3 & 60.6 \\
\hline $\mathrm{KI}$ & 5.1 & 2.7 & 72.0 \\
\hline $\mathrm{RbF}$ & 6.5 & 1.9 & 35.0 \\
\hline $\mathrm{RbCl}$ & 4.9 & 2.2 & 57.8 \\
\hline $\mathrm{RbBr}$ & 4.9 & 2.3 & 78.8 \\
\hline $\mathrm{RbI}$ & 5.5 & 2.6 & 97.1 \\
\hline $\mathrm{CsCl}$ & 7.2 & 2.6 & 60.6 \\
\hline $\mathrm{CsBr}$ & 6.5 & 2.8 & 89.4 \\
\hline CsI & 5.65 & 3.0 & 117.7 \\
\hline $\mathrm{MgO}$ & 9.8 & 2.95 & 13.7 \\
\hline
\end{tabular}


It is interesting to plot the mean-free path between collisions $\langle\lambda\rangle$ as a function of electron energy for a few alkali halides, ranging from the heaviest to the lightest. The computation is done by taking $\mathrm{m}^{*} / \mathrm{m}=1, \mathrm{~T}=300^{\circ} \mathrm{K}$, and again, the basic data from Bak. ${ }^{52}$ The results from computing

$$
\langle\lambda\rangle=\frac{\left(26 / \mathrm{m}^{*}\right)^{1 / 2}}{\mathrm{w}_{\overrightarrow{\mathrm{k}}}^{-}+\mathrm{w}_{\mathrm{k}}^{+}}
$$

are shown in Fig. 8.

One characteristic of these results soon becomes apparent at the lower energies: $\langle\lambda\rangle$ is comparable to the lattice constant and one cannot help feeling uncertain about a theory which is based on the equations of motion of a macroscopic polarization. An electron moving with a mean-free path comparable to the lattice constant probably would see a structure in the polarization field. It is not clear whether this would result in a higher or lower collision rate.

From a macroscopic point of view, it has been pointed out by Harrison 59 that when the losses are high, the results obtained by neglecting the imaginary part of the dielectric constant in the coupling between an externally applied field and the lattice can lead to results for the energy losses which are too high.

One simple way to see this effect consists in considering a one-dimensional wave packet of charge " $\rho "$ travelling in a crystal. A Fourier analysis would yield a function $\phi(k)$ strongly peaked about a value $k_{0}$, such that

$$
\rho(x)=\int_{-\infty}^{\infty} \phi(k) e^{i(k-\omega t)} d k, \quad \text { where } \omega=\frac{k^{2}}{2 m} \text {. }
$$




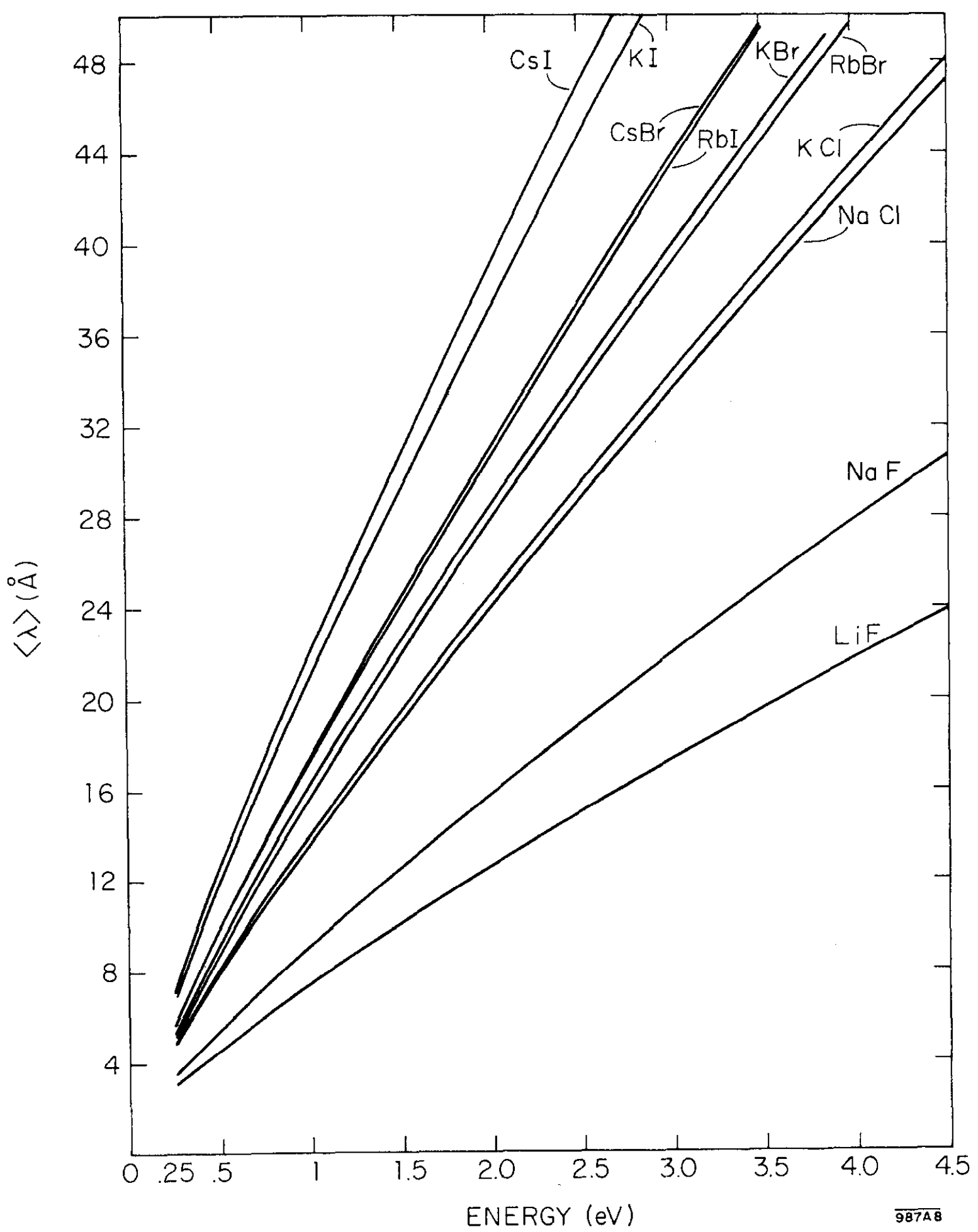

FIG. 8--Mean free path vs. electron energy for several alkali halides 
If we take one component with wavenumber $\mathrm{k}$ out of the packet, the electronic field $E_{0}$ due to the charge will be

$$
E_{0}=\frac{-i 4 \pi}{k} \phi(k) e^{i(k x-\omega t)} .
$$

This field can be considered to be an externally applied field, generating an internal field $E$ in the crystal determined by the complex dielectric constant $\epsilon_{1}-i \epsilon_{2}$,

$$
E=\frac{E_{0}}{\epsilon_{1}-i \epsilon_{2}}=\frac{-i \epsilon_{1}+\epsilon_{2}}{\epsilon_{1}^{2}+\epsilon_{2}^{2}} \frac{4 \pi}{k} \phi(k) e^{i(k x-\omega t)} .
$$

The current due to the charge flow is given by

$$
\nabla \cdot \overrightarrow{\mathrm{J}}=-\frac{\partial \rho}{\partial \mathrm{t}}
$$

or

$$
J=\frac{\omega}{k} \phi(k) e^{i(k x-\omega t)}
$$

and the power transfer per unit volume in the crystal is then

$$
\frac{1}{2} \operatorname{Re}\left\{\overrightarrow{J^{*}} \cdot \vec{E}\right\}
$$

or

$$
P=\frac{\omega 4 \pi}{k^{2}} \phi^{2} \frac{\epsilon_{2}}{\epsilon_{1}^{2}+\epsilon_{2}^{2}}
$$

Using $\omega=\pi k^{2} / 2 \mathrm{~m}$, the total power transfer for the packet in the crystal of unity cross sectional area is

$$
\mathrm{P}_{\mathrm{t}}=\frac{\nVdash 4 \pi}{2 \mathrm{~m}} \int_{-\infty}^{\infty} \frac{\epsilon_{2}(\mathrm{k})}{\epsilon_{1}^{2}(\mathrm{k})+\epsilon_{2}^{2}(\mathrm{k})} \phi(\mathrm{k}) \mathrm{dk}
$$

An examination of this result in comparison to Eqs. (11) and (13), realizing that $1 / \gamma$ stands basically for $1 / \epsilon_{1}$ in the frequencies of interest, shows that the whole 
approach by perturbation theory has been based on a relationship of the form

$$
\mathrm{E}=\frac{\mathrm{E}_{0}}{\epsilon_{1}} \text {. }
$$

In fact, Eq. (5) corresponds to an undamped harmonic oscillator.

The energy loss derived from Eqs. (12) and (14) is proportional to some value of $\epsilon_{2}$ and it appears that if this value for $\epsilon_{2}$ is sufficiently large (as shown by a large scattering rate) it has to be included in the ccmplex dielectric constant, and calculations be carried out for a self-consistent solution.

The above argument may indicate that the very short values of $\langle\lambda\rangle$ in Fig. 8 are not correct and should be longer. We shall return to this point in making comparisons with experiments.

Returning to the results of the perturbation theory, the angular distribution of the scattering can easily be found from Eqs. (11) and (13). For the case of phonon annihilation,

$$
\frac{4 \pi e^{2} m^{*} n_{q}}{\hbar^{2} \gamma \omega} \frac{1}{k} \frac{1}{q} d q
$$

is the rate of scattering into a ring of area $2 \pi \mathrm{k}^{{ }^{2}} \sin \theta \mathrm{d} \theta$, with the relationships

$$
\begin{aligned}
& \mathrm{q}^{2}=\mathrm{k}^{2}+\mathrm{k}^{2}-2 \mathrm{kk}^{\prime} \cos \theta \\
& \mathrm{qdq}=\mathrm{kk}^{\prime} \sin \theta \mathrm{d} \theta
\end{aligned}
$$

Substituting into the above expression we find that the probability of scattering into a ring between $\theta$ and $\theta+\mathrm{d} \theta$,

$$
\mathrm{P}(\theta) \mathrm{d} \theta \propto \frac{\mathrm{k}^{\prime} \sin \theta \mathrm{d} \theta}{\mathrm{k}^{2}+\mathrm{k}^{\prime^{2}}-2 \mathrm{kk}^{\prime} \cos \theta} \quad \text { where } \quad \mathrm{k}^{2}=\mathrm{k}^{2}+\frac{2 \mathrm{~m}^{*} \omega}{\mathrm{h}}
$$

For phonon creation, $\mathrm{P}(\theta) \mathrm{d} \theta$ has the same form, but

$$
\mathrm{k}^{2}=\mathrm{k}^{2}-\frac{2 \mathrm{~m}^{*} \omega}{\mathrm{h}}
$$


The function $\mathrm{P}(\theta) \mathrm{d} \theta$ normalized to unity area is shown in Fig. 9 for the parameters of CsI and electron energies between .25 and $5 \mathrm{eV}$. An arrow indicates the angle $\bar{\theta}$ at which the probability of an electron being scattered to $\theta<\bar{\theta}$ is . 5 . Although it is clear that the scattering is predominantly forward, particularly at the higher energies, there is a substantial probability for moderately wide angle scattering. In thick films, the effects of multiple scattering may not be negligible and the assumption of only forward scattering of Khokley and van Vliet ${ }^{38,39}$ is therefore not completely appropriate for a detailed study. In particular such an assumption would not offer any explanation for the quantum yields above .5 observed in photoemission experiments with alkali halides. 48,49

\section{The Boltzmann Transport Equation}

A Boltzmann transport equation for the secondary emission problem based on the electron-phonon scattering characteristics described in the previous section has been developed but found impossible to solve. The complete equation will be written below and the difficulties found in the solution will be pointed out. Without making any approximations in the scattering terms, we obtain for a given primary energy, assuming that the only spatial variations are in the $\mathrm{x}$-direction. 


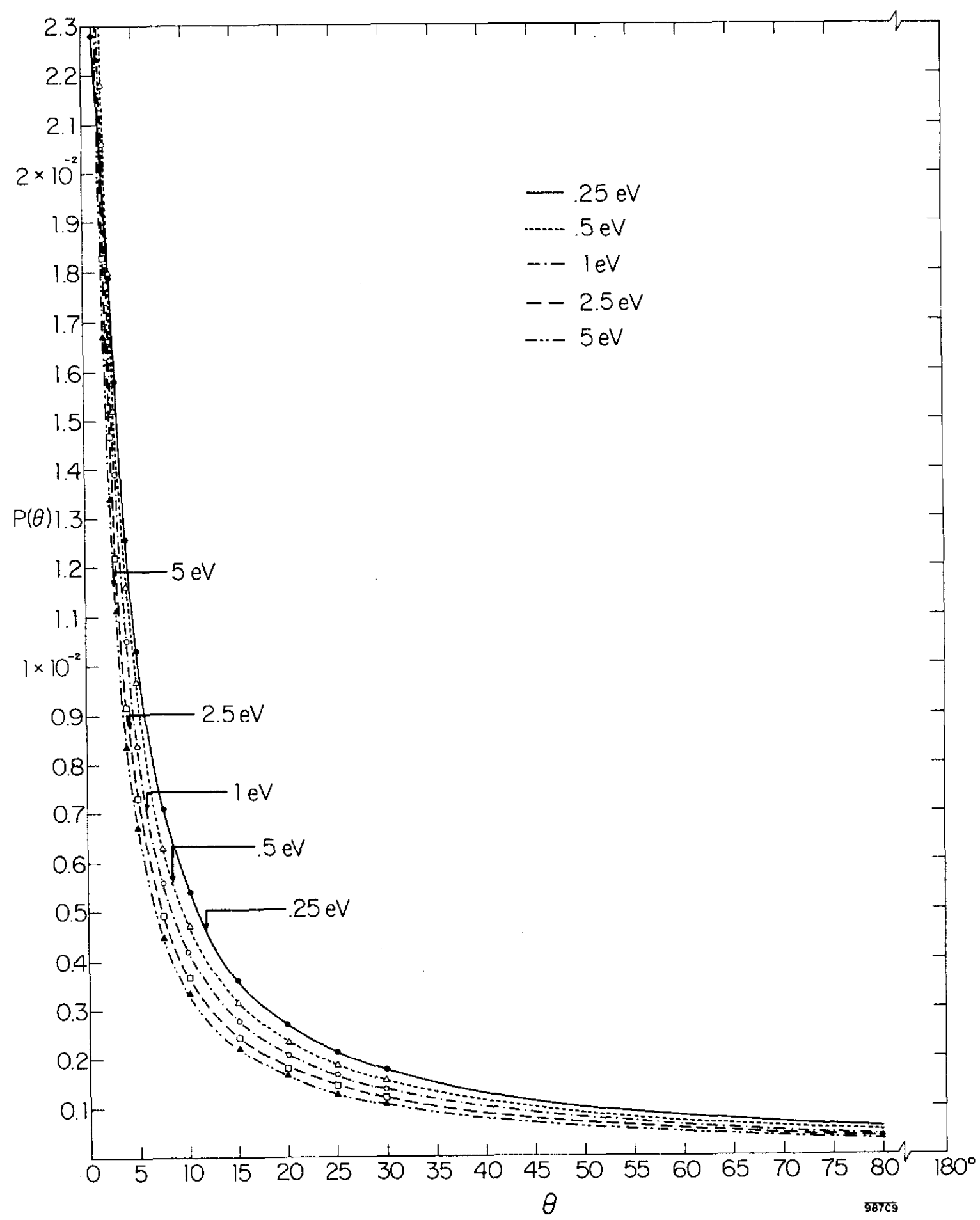

FIG. 9--Scattering angular probability distribution for CsI, phonon annihilation 


$$
\begin{aligned}
& \frac{\partial f(x, \delta, \theta)}{\partial x}=\frac{1}{\left(\frac{2 e}{m^{*}(\varepsilon)^{1 / 2} \cos \theta}\right.}\left\{G(E, x, \theta)-\frac{f(x, E, \theta)}{\tau}-\right. \\
& \left.-\mathrm{f}(\mathrm{x}, \hat{E}, \theta) \operatorname{An}_{\mathrm{q}} \frac{1}{\sqrt{\mathscr{\delta}}} \log \left(\frac{\left(1+\frac{\delta}{\delta}\right)^{1 / 2}+1}{\left(1+\frac{\delta}{\delta}\right)^{1 / 2}-1}\right)-\mathrm{f}(\mathrm{x}, \mathscr{E}, \theta) \mathrm{A}_{(\mathrm{n}}+1\right) \frac{1}{\sqrt{\mathscr{\delta}}} \log \left(\frac{1+\left(1-\frac{\delta}{3}\right)^{1 / 2}}{1-\left(1-\frac{\delta}{\delta}\right)^{1 / 2}}\right)+ \\
& +\int_{\theta^{\prime}=0}^{\pi} \mathrm{f}\left(\mathrm{x}, \mathscr{E}^{\mathrm{v}}-\delta, \theta^{\prime}\right) \operatorname{An}_{\mathrm{q}}\left(\frac{\tilde{E}-\delta}{\xi}\right)^{3 / 2} \\
& \frac{(\delta-\delta)^{1 / 2} \sin \theta^{\prime} d \theta^{\prime}}{\left[\left(2 \delta-\delta-2\left(\delta^{2}-\delta \delta\right)^{1 / 2} \cos \theta \cos \theta^{\prime}\right)^{2}-\left(2\left(\delta^{2}-\delta \delta\right)^{1 / 2} \sin \theta \sin \theta^{\prime}\right)^{2}\right]^{1 / 2}}+ \\
& +\int_{\theta^{\prime}=0}^{\pi} f\left(x, \delta-\delta, \theta^{\prime}\right) A\left(n_{q}+1\right)\left(\frac{\delta+\delta}{\delta}\right)^{3 / 2} \\
& \left(\mathscr{E}^{\prime}+\delta\right)^{1 / 2} \sin \theta \sin \theta^{\prime} \mathrm{d} \theta^{\prime} \\
& \text { - } \left.\left[\left(2 \delta+\delta-2\left(\delta^{2}+\delta \delta\right)^{1 / 2} \cos \theta \cos \theta^{\prime}\right)^{2}-\left(2\left(\delta^{2}+\delta \delta\right)^{1 / 2} \sin \theta \sin \theta^{\prime}\right)^{2}\right]^{1 / 2}\right\}
\end{aligned}
$$

where

$$
A=\frac{1}{\left(\frac{2 e ́}{m^{*}}\right)^{1 / 2}} \frac{4 \pi \mathrm{e}^{2}}{\hbar \gamma \omega} \quad \text { and } \quad \delta=\hbar \omega \text {. }
$$

The first term in the righthand side corresponds to an increase in the distribution function due to generation of electrons in a thickness $d x$ at $x$, as described in part $\mathrm{C} 5$ of this report. The second term corresponds to a loss of electrons by some trapping mechanism, with lifetime $\tau$. The third and fourth terms correspond 
to the scattering out of electrons from an elementary volume in $(\xi, \theta)$ space to all other allowed energies and angles for the phonon annihilation and creations cases, respectively. Finally, the last two terms correspond to scattering into the elementary volume in $(\mathcal{E}, \theta)$ space from all other allowed energies and angles.

Since it appeared impossible to expand the scattering terms in a small number of spherical harmonics and still preserve their character, a direct analytic solution had to be given up. A solution for a $\delta$-function generation function in a way similar to the one used by Baraff ${ }^{53}$ was also tried but difficulties also developed due to the complex form of the "scattering in" terms.

Finally, an attempt was made to solve the differential equation by numerical integration. In spite of considerable care in the methods used, a very strong instability developed in the solution due to the fact that the "scattering in" and "scattering out" terms are both very large but their difference small. Errors introduced in the evaluation of the integrals over $\theta^{\prime}$ grew exponentially in very few steps and no way was found to control the solution within the memory capabilities of the computer used (IBM 7090).

Fortunately, the problem is just ideally suited for a Monte Carlo solution by the method of direct simulation.

\section{Monte Carlo Solution}

The Monte Carlo direct simulation method consists in assuming that one particle is generated with a particular initial position, direction, and energy at time $t_{0}$ and moves freely for a certain length of time $t_{1}$ until it suffers a collision. The particle continues then with a new direction and energy from a new initial position determined by $t_{1}$ and the old parameters. This process is repeated until the particle reaches the condition which we are trying to study, in the present case, until the electron gets absorbed in the film or comes out from it. A random variable which 
is properly weighted by a function obtained from the physical process under consideration determines the length of the $t_{i}$ 's and the change in energy and angle at each collision. Usually a fairly large number of particle tracks have to be followed in order to establish a result with good statistical certainty.

Given an average scattering rate $\mathrm{W}(6)$ such that $1 / \mathrm{W}=\tau$ is the mean-time between collisions, it will now be shown that the probability that a particle be scattered between any time $t$ and $t+\Delta t$ is approximately $\Delta t / \tau$ for $\Delta t \ll \tau$. Start by considering a large number $\mathrm{N}_{0}$ of particles. After a short time $\Delta t$, the fraction $\Delta \mathrm{N}$ of scattered particles will be $\Delta \mathrm{N} / \mathrm{N}_{0}=\Delta \mathrm{t} / \tau$. In the limit $\mathrm{dN} / \mathrm{dt}=\mathrm{N}_{0} / \tau$, or

$$
\frac{N}{N_{0}}=1-e^{-t / T}=F(t)
$$

$F(t)$ is a distribution function giving the probability that a particle has been scattered after a time $t$. On the other hand, if we postulate that the scattering probability for one particle between $t$ and $t+\Delta t$ is $\Delta t / \tau$, then

and

$$
P_{S}(0, \Delta t)=\frac{\Delta t}{T}=p
$$

$$
\begin{aligned}
& P_{S}(\Delta t, 2 \Delta t)=(1-p) p \\
& \dot{P}_{S} n \Delta t,(n+1) \Delta t=(1-p)^{n} p
\end{aligned}
$$

and the (integral) probability that a particle is scattered after some time $m \Delta t$ is given by the sum of the above:

$$
\mathrm{p}\left(1+(1-\mathrm{p})+(1-\mathrm{p})^{2}+\ldots(1-\mathrm{p})^{\mathrm{m}-1}\right)=\frac{\mathrm{p}\left(1-(1-\mathrm{p})^{\mathrm{m}}\right)}{1-(1-\mathrm{p})}=1-(1-\mathrm{p})^{\mathrm{m}}
$$

If $p$ is small enough,

$$
1-(1-p)^{m} \simeq 1-e^{-p m}=1-e^{-(\Delta t / \tau)(t / \Delta t)}=1-e^{-t / \tau}
$$

so that the two solutions are equivalent. 
This indicates the nature of the weighted random process which will determine the occurrence of a collision. For an electron which at some time $\mathrm{t}$ has an energy $\dot{b}$, Eqs. (12) and (14) determine the mean-free times $\tau^{-}\left(\mathscr{G}^{\circ}\right)$ and $\tau^{+}(\mathscr{E})$. Choosing an interval $\Delta \mathrm{t} \ll \tau^{-}$or $\tau^{+}$, the probability that the electron suffers a collision in that interval of time is $\left(\Delta t / \tau^{-}\right)+\left(\Delta t / \tau^{+}\right)$. Let $\xi^{\prime}$ s be numbers from a set of uniformly distributed random numbers between 0 and 1. For every interval of time $\Delta t$ of the life of the electron, we draw one random number. If $0 \leq \xi<\Delta t / \tau^{-}$, the electron suffers a collision with the annihilation of one phonon. If $\Delta \mathrm{t} / \tau^{-} \leq \xi<\left(\Delta \mathrm{t} / \mathrm{T}^{-}\right)+\left(\Delta \mathrm{t} / \mathrm{T}^{+}\right)$, the electron suffers a collision with phonon creation. If $\left(\Delta t / \tau^{-}\right)+\left(\Delta t / \tau^{+}\right) \leq \xi<1$, there is no collision and the electron follows with the same energy and direction for a time $\Delta$ t.

Whenever there is a collision, the electron is assumed to emerge instantaneously with a new energy $\delta \pm \delta$, depending on the type of collision. The change in $\theta$ with respect to the old direction is to be computed by properly weighing random numbers so that their distribution becomes the one given by Eqs. (15) and (16). This is done by inversion of the distributions. ${ }^{54}$ Let $\theta^{\prime} \quad$ be the random angle with distribution given by Eq. (15), while $\xi$ is uniformly distributed between 0 and 1 . After drawing a new $\xi$, we require

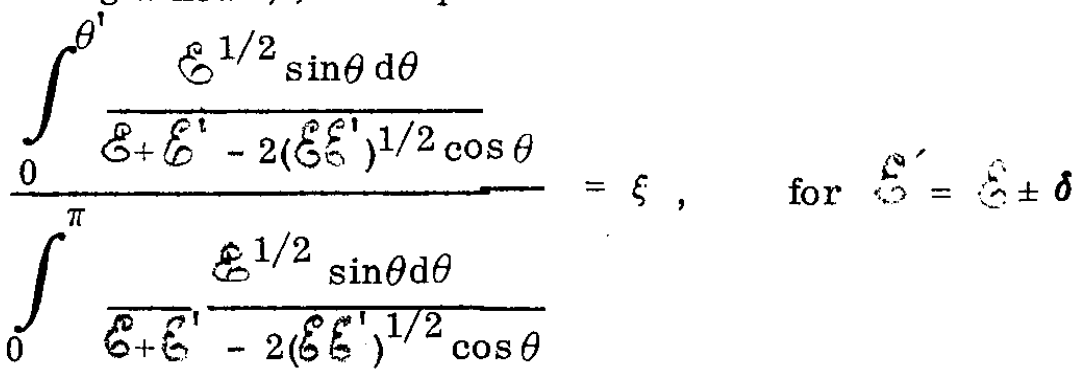

$$
\begin{aligned}
& \text { or } \xi \log \frac{E+E^{\prime}+2\left(E E^{\prime}\right)^{1 / 2}}{E+E^{\prime}-2(E G)^{1 / 2}}=\log \frac{E^{\prime}+E^{\prime}-2\left(E E^{\prime}\right)^{1 / 2} \cos \theta^{\prime}}{E+E^{\prime}-2\left(E^{\prime}\right)^{1 / 2}} \text {. }
\end{aligned}
$$

Solving for $\cos \theta^{\prime}$, we obtain

$$
\cos \theta^{\prime}=\frac{\xi^{\prime}+\xi^{\prime}}{2\left(\xi \xi^{\prime}\right)^{1 / 2}}\left(1-B^{\xi}\right)+B^{\xi} \quad \text { where } \quad \text { B }=\frac{\xi^{\prime} \xi^{\prime}+2\left(\xi^{\prime}\right)^{1 / 2}}{\xi+\xi^{\prime}-2\left(\xi^{\prime}\right)^{1 / 2}} .
$$


This procedure has been carefully tested and found to give distribution functions which, when plotted, are indistinguishable from the ones given by Eqs.(14) and (15) after a few thousand random numbers have been sampled. The computations for $\cos \theta^{\prime}$ have to be carried out in double precision when an IBM $360 / 75$ computer is used, otherwise a short computer "word" results in high scattering angles never being reached.

The change in $\phi$ with respect to the old direction is done by sampling a distribution function which is uniformly distributed between 0 and $2 \pi$.

When an interaction occurs, an electron which had a momentum $(p, \theta, \phi)$ with respect to the forward direction, suffers a change in the three components of momentum. If the sampling of the above distributions gives a change by $\theta^{\prime}, \phi^{\dagger}$, the new momentum vector is found in the following way:

We set a laboratory frame with $\mathrm{p}_{\mathrm{z}}$ in the forward direction. The frame is rotated about $p_{z}$ until $\phi_{\text {old }}=0$, for convenience (see Fig. 10a). Then, set up a new coordinate system by a rotation by $\theta$ about the $\mathrm{p}_{\mathrm{y}}$ axis (Fig. $10 \mathrm{~b}$ ) so that $\mathrm{p}_{\mathrm{z}}$ is along $\overrightarrow{\mathrm{p}}$. The new scattering by $\theta^{\prime}$ and $\phi^{\prime}$ is with respect to the $\mathrm{p}_{\mathrm{x}}^{\prime}, \mathrm{p}_{\mathrm{y}}^{\prime}, \mathrm{p}_{\mathrm{z}}^{\prime}$ coordinates.

The new $\vec{p}_{\text {new }}$ expressed in the laboratory frame is given by the transformation $\left(\begin{array}{c}p_{x \text { new }} \\ p_{\text {y new }} \\ p_{z_{\text {nnew }}}\end{array}\right)=\left(\begin{array}{ccc}\cos \theta & 0 & \sin \theta \\ 0 & 1 & 0 \\ \sin \theta & 0 & \cos \theta\end{array}\right)\left(\begin{array}{c}p^{\prime} \sin \theta^{\prime} \cos \phi^{\prime} \\ p^{\prime} \sin \theta^{\prime} \sin \phi^{\prime} \\ p^{\prime} \cos \theta^{\prime}\end{array}\right)$

Since we are only interested in the displacement of the electrons in the forward direction, we obtain

$$
\mathrm{p}_{\mathrm{z} \text { new }}=\mathrm{p}^{\prime}\left(\cos \theta^{\prime} \cos \theta-\sin \theta^{\prime} \cos \phi^{\prime} \sin \theta\right)
$$



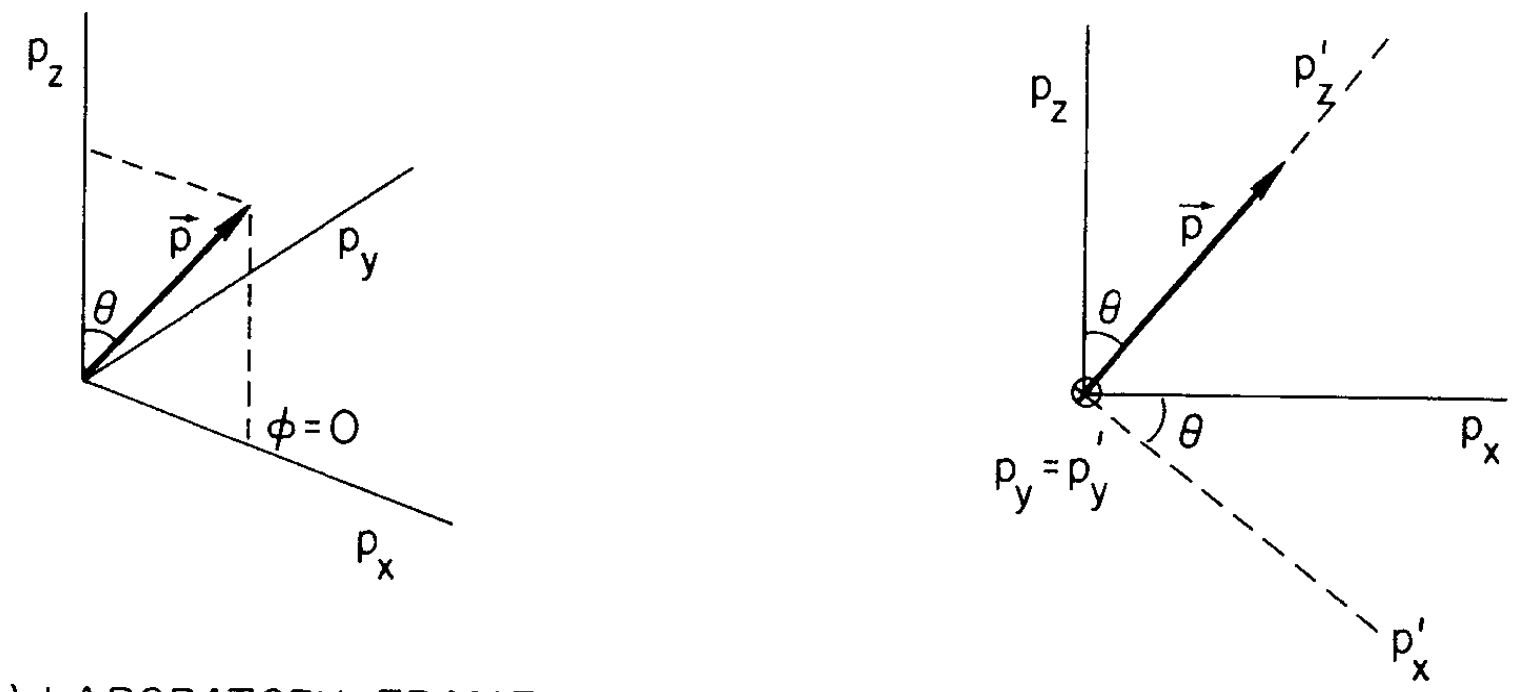

(a) LABORATORY FRAME

(b) ROTATED FRAME

$\overline{987 A 10}$

FIG. 10--Coordinates for the computation of new momentum coordinates upon scattering 
At this point one can redefine the laboratory frame $p_{x}, p_{y}, p_{z}$ so as to make $\phi=0$ again. The new $\theta$ to start the computation of the new trajectory is therefore given by

$$
\cos \theta_{\text {new }}=\cos \theta^{\prime} \cos \theta_{\text {old }}-\sin \theta^{\prime} \cos \phi^{\prime} \sin \theta_{\text {old }} .
$$

The next question to be considered is the behavior of an electron reaching the surface of the crystal. The customary approach is that of assuming a potential barrier at the surface which acts on the nor mal component of velocity. This is considerably more reasonable for the treatment of electron emission from single crystals than for the case of evaporated films consisting of many small randomly oriented crystals. In the latter case, only a simpler form of barrier seems justifiable. From UV absorption, photoemission and/or photoconduction in alkali halide films, one can obtain reasonably good information about the location of the bottom of the conduction band for a good number of materials. Also from photoemission data, one can obtain the approximate location of the vacuum level. It must be noted that the disturbances associated with exciton enhanced emission make accurate determinations difficult.

Table 6 contains the available information from the literature. 
TABLE 6

\begin{tabular}{|c|c|c|c|c|c|c|}
\hline \multirow[t]{2}{*}{ Material } & \multicolumn{3}{|c|}{ Bottom of Conduction Band eV } & \multicolumn{2}{|c|}{ Vacuum level eV } & Electron affinity eV \\
\hline & Ref. 55 & Ref. 56 & Ref. 49 & Ref. 55 & Ref. 49 & \\
\hline $\mathrm{KF}$ & 10.9 & - & - & 10.4 & & $\simeq 0$ (or $<0$ \\
\hline $\mathrm{RbF}$ & $\simeq 10.4$ & - & - & - & & - \\
\hline $\mathrm{CsF}$ & 10.0 & - & - & - & & - \\
\hline $\mathrm{NaCl}$ & 8.6 & - & - & 8.5 & & $0($ or $<0)$ \\
\hline $\mathrm{KCl}$ & 8.5 & 8.9 & - & 8.7 & & $0($ or $<0)$ \\
\hline $\mathrm{RbCl}$ & 8.2 & 8.5 & - & - & & - \\
\hline $\mathrm{CsCl}$ & - & - & 7.4 & - & $\simeq 7.4$ & $\simeq \quad 0$ \\
\hline $\mathrm{NaBr}$ & $\simeq 7.7$ & - & - & - & & - \\
\hline $\mathrm{KBr}$ & 7.8 & 8.00 & - & 8.1 & & $\simeq-1$ \\
\hline $\mathrm{RbBr}$ & $\simeq 7.7$ & - & - & - & & - \\
\hline $\mathrm{CsBr}$ & - & - & 7.4 & - & $\simeq 7.4$ & $\simeq 0$ \\
\hline LiI & $\simeq 5.9$ & - & - & 7.3 & & - \\
\hline $\mathrm{NaI}$ & 5.8 & - & - & 7.3 & & - \\
\hline $\mathrm{KI}$ & 6.2 & 6.31 & - & 7.3 & & $\simeq 1 \mathrm{eV}$ \\
\hline $\mathrm{RbI}$ & 6.1 & 6.26 & - & 7.3 & & $\simeq 1 \mathrm{eV}$ \\
\hline CsI & 6.3 & 6.37 & $\simeq 6.0$ & 6.4 & $\simeq 6.3$ & $0($ or $<0)$ \\
\hline
\end{tabular}

The values for the energy at bottom of conduction band (referred to the top of the valance band) given in the first column of Table 6 , were obtained by Eby, Teegarden and Dutton ${ }^{55}$ by assuming that an absorption shoulder in the spectrum of the salts was due to the onset of band-to-band transitions. Recently, Teegarden and Baldini ${ }^{56}$ have modified the previous results after realizing that the absorption shoulder is a poorly resolved series of excitonic peaks, converging 
to the onset of the band-to-band transitions. Their figures, given in the second column, are from photoconduction results or from a limit of an excitonic series. The values on the third column have been obtained by Kholikowski ${ }^{49}$ by an extrapolation of the leading edge of the energy distribution of photoemitted electrons, which should move by $\Delta E=\Delta h \nu_{0}$ when the bombarding light changes in energy by $\Delta h \nu_{0}$.

The values for the vacuum level given by Ref. 55 correspond to the energy at which photoelectric quantum efficiency reaches $10^{-3}$ at $300^{\circ} \mathrm{K}$ (data obtained from Taft and Phillip ${ }^{57}$ ). For the results from Ref. 49, approximately the same criterion has been used, except for $\mathrm{CsBr}$ where the figure corresponding to sharp rise in quantum yield has been used.

In calculating the electron affinity, it is clear that only an estimate can be made due to uncertainties in relating different measurements. It is apparent, however, that the electron affinity is very nearly zero for the alkali halides with available data, except in two cases (KI and $\mathrm{RbI}$ ) where the results indicate an electron affinity of approximately $1 \mathrm{eV}$. It seems likely that this is in error, though, as there is no special characteristic of these two materials which makes them different from the rest.

It is therefore proposed to treat the existence of a small surface barrier as follows: During the calculation of electron trajectories there will be an energy $\mathscr{\sigma}_{\min }$ such that if any electron reaches $\delta<\varepsilon_{\text {min }}$, it will be considered absorbed (eventually recombined) so that it will not contribute to secondary emission. $E_{\min }$ will be taken to be approximately $.05 \mathrm{eV}$ for all materials. It will become evident later that any reasonable choice for this figure yields approximately the same results.

Since the Monte Carlo method requires a certain number of trials in order to establish a result with a required certainty, it is necessary to find a criterion for 
deciding automatically within the computation when enough trials have been made. The method to be used is the one described by Cashwell and Everett. 58 Let "p" be the true probability that electrons generated with certain characteristics will escape from a film. After carrying out $\mathrm{N}$ trials, it is found that $\mathrm{M}$ electrons escaped. Then, the ratio of the number of sequences of $N$ trials resulting in a ratio $M / N$ satisfying the inequality

$$
\left|\frac{M}{N}-p\right|<\epsilon
$$

to the totality of all possible sequences of $\mathrm{N}$ trials is, approximately,

$$
f(t)=\operatorname{erf}(t / \sqrt{2})
$$

where

$$
t=\epsilon(N / p(1-p))^{1 / 2}
$$

and

$$
\operatorname{erf}(x)=\frac{2}{\sqrt{\pi}} \int_{0}^{x} e^{-x^{2}} d x
$$

As an example, we may require that if we carried out $N_{1}$ trials a large number of times, $90 \%$ of the times we come out with $\left|\left(\mathrm{M}_{1} / \mathrm{N}_{1}\right)-\mathrm{p}\right|<\epsilon_{1}$.

Then, we set . $9=\operatorname{erf}(t / \sqrt{2})$, solve for $t$, and approximating $p \approx M / N$ as obtained from $\mathrm{N}$ trials, we can find the required $\mathrm{N}_{1}$. In general, the computations to be reported have been carried out to $95 \%$ certainty of an error less than the one indicated in the individual cases.

This method can be used as well to evaluate the certainty of energy and angular distributions of the outcoming electrons. During the computations, the energy and angle of an outcoming electron are recorded in a two-dimensional multichannel array by putting one count in the corresponding cell. At the end of the computation, energy and angular distributions are obtained separately by summations, and correlations between energy and angle can be obtained. If one lets " $p$ " be the true probability of 
electrons falling in a particular slot of energy (and/or angle), and $M$ be the number of counts obtained in that slot out of $\mathrm{N}$ electrons, the same scheme as above can be used. Clearly, in order to obtain good statistics in these distributions a larger number of trials will be needed than when only probability of escape is computed.

\section{Results of Computations}

Computations for films of different alkali halides have been carried out. Films with a thickness of $250 \AA$ have been considered throughout since this thickness gives sufficient information as to the behavior of secondary electrons in films as well as in thicker solids. Film coordinates for the computations are shown in Fig. 11. The films have been considered symmetric for the computations of the individual escape probabilities to be presented here, i.e., the proba bility of escape through the exit face of an electron generated backwards at some distance from the exit surface is equal to the probability of escape through the substrate face of an electron generated forward at the same distance from the substrate. This cuts the number of computations by a factor of 2 and it is done under the assumption that a metallic backing will act as a sink for electrons with identical characteristics as vacuum.

The Monte Carlo method allows the obtaining of a great variety of information. The expenditure of computer time can be, however, quite considerable and it is necessary to conduct the calculations in such a form that the most relevant results are obtained with reasonable accuracy. For the problem of secondary emission, and also for photoemission, the probabilities of escape for electrons generated with some given energy, at a given depth, with an isotropic distribution of velocities, seems to be the most important one. The calculation of exit energy distributions for electrons generated at some particular energies, and their mean energy loss per unit depth can be obtained as a by-product of the previous problem without much added computation. Also the exit angular distribution for electrons which started 


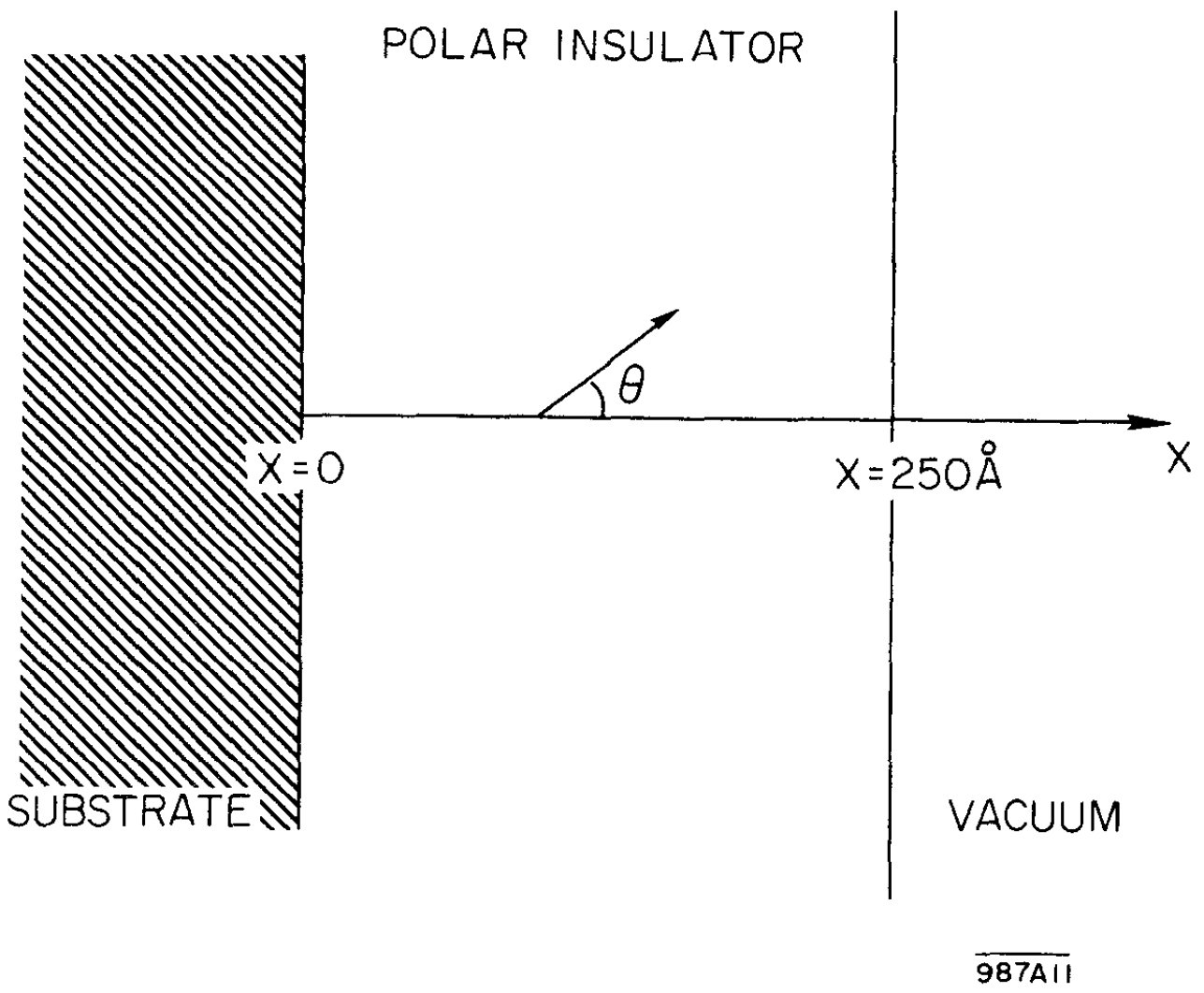

FIG. 11--Film coordinates for computations of escape probabilities 
at some given angles, and some energy-angular correlations, are easy to obtain. However, the overall computation of energy and/or angular distributions for the complete phenomenon of secondary emission requires a large inc rease in the number of computations, as a tight "mesh" of initial energies and angles would be required. Computations carried out indicate that it is possible to obtain good solutions for the escape probabilities and individual energy and angular distributions for $250 \AA$ films in approximately four hours on the IBM 7090 computer. This also gives some information on overall distributions, as will be shown in Chapter 3. Computations carried out on the IBM $360 / 75$ are faster by a factor of approximately 5 .

In the computations to be reported, the film is divided into a 3 -dimensional mesh with 5 initial locations of the generated electrons: $x=25,75,125,175$,

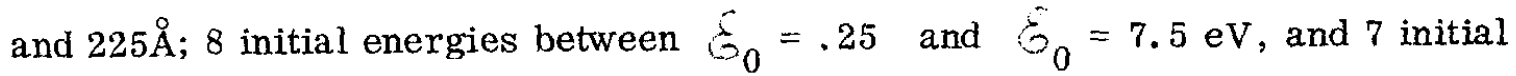
angles: $\theta=0,30,60,90,120,150$, and $180^{\circ}$. This results in 280 calculations, e ach requiring between 200 and 800 complete trajectories in order to obtain escape probabilities with an estimated error of approximately \pm 0.03 with $95 \%$ certainty.

Four alkali halides have been selected for study: cesium iodide representing materials with long wavelength in the longitudinal optical modes and having high yield; potassium chloride for medium wavelength materials and moderate gain; sodium fluoride for short wavelength and moderate gain; and lithium fluoride for even shorter wavelength and low gain. The results obtained for CsI will be chosen to illustrate some of the detailed information obtainable from the Monte Carlo calculations.

a. Results for Cesium Iodide, $\mathrm{m}^{*} / \mathrm{m}=1, \mathrm{~T}=300^{\circ} \mathrm{K}$

Figure 12, a through $\mathrm{g}$, shows the probabilities of escape $\mathrm{P}\left(\mathscr{E}_{0}, \mathrm{x}, \theta\right)$ through the forward exit surface of electrons generated with an energy ${ }_{0}^{e}$, at a position $\mathrm{x}$, with a direction $\theta$ with respect to the forward direction. The range 

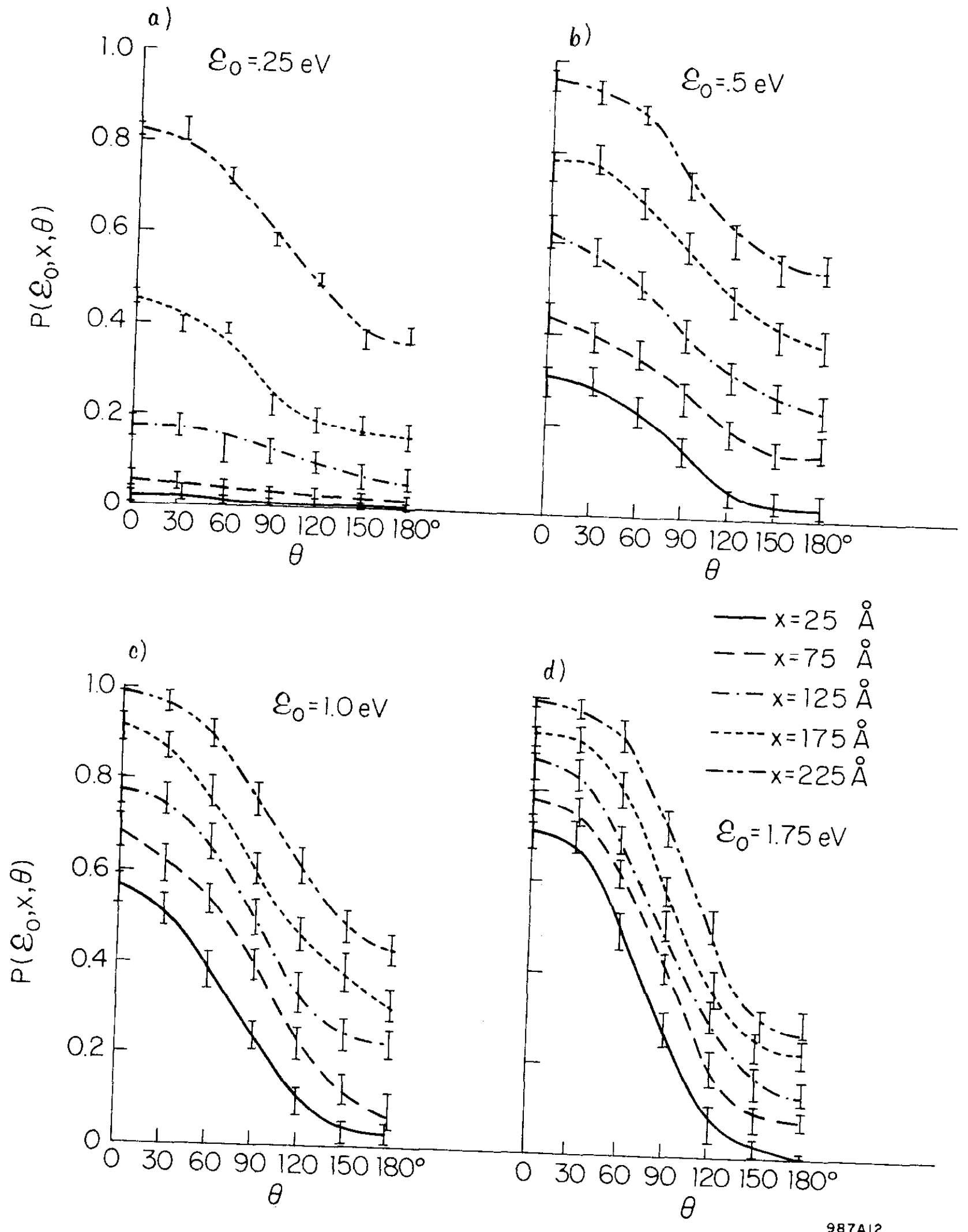

FIG. 12--Probabilities of escape for electrons generated at a position $x$ in a $250 \AA$ film of CsI, with initial energy $s_{0}$ and initial angle $\theta$ 

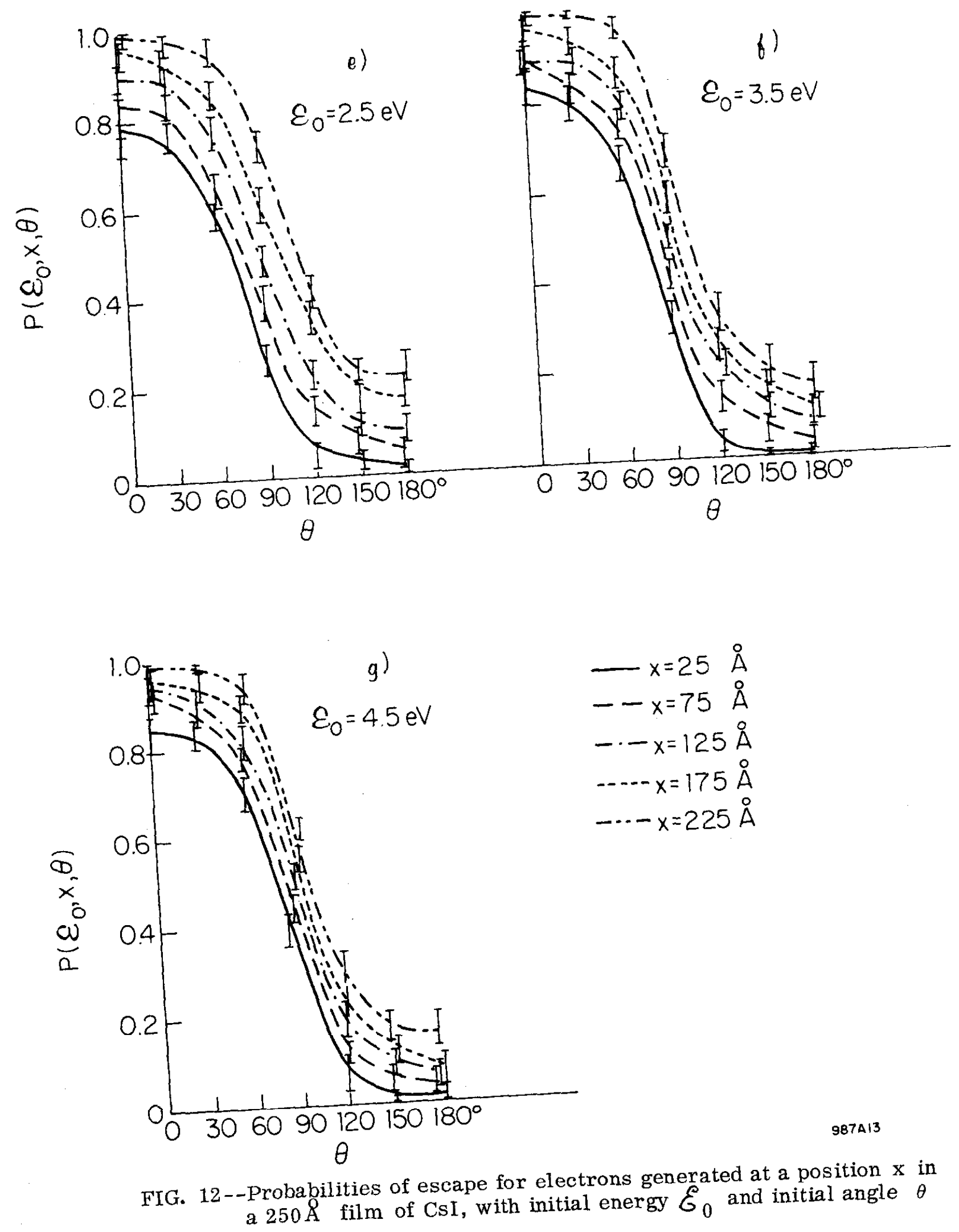
of errors shown is for $95 \%$ certainty in each computation, as discussed above. The effect of scattering other than forward is very marked, particularly at the lower energies where the probability of escape of electrons generated at $\theta>90^{\circ}$ is very substantial.

When the electrons are generated isotropically, the escape probability averaged over all angles can be obtained from

$$
\mathrm{P}\left(\tilde{\varepsilon}_{0}, \mathrm{x}\right)=\frac{1}{2} \int_{0}^{\pi} \mathrm{P}\left(\tilde{\varepsilon}_{0}, \mathrm{x}, \theta\right) \sin \theta \mathrm{d} \theta
$$

Having divided $\theta$ into six sections of $30^{\circ}$ each, the above integral can be evaluated approximately by a summation. The results are shown in Fig. 13; the estimated statistical errors are approximately \pm .035 .

The escape probability for . $25 \mathrm{eV}$ electrons has a definitely exponential character. When plotted on a semilogarithmic scale (see Fig. 14a) it results in a straight line corresponding to a characteristic escape length $\mathrm{L}_{\mathrm{s}} \simeq 62 \AA$. However, for electrons generated near the substrate side of the film (low $\mathrm{x}$ ) the escape probability is lower than the one given by the exponential, reflecting the fact that a sink for electrons exists at $\mathrm{x}=0$. This can be understood physically by consideration of the non-forward scattering suffered by the electrons arriving at $x=0$ with a component of velocity in the $-x$ direction. If there were more alkali halide material for $\mathrm{x}<0$, such electrons would have a chance of being scattered into the forward direction and contribute to secondary emission. The rest of the curves are best fitted by straight lines and this is attributed again to the presence of the sink for electrons at $\mathrm{x}=0$. The effect becomes important when an exponential characteristic length $\mathrm{L}_{S}$ would be of a magnitude comparable to the dimensions of the film. For example, for $\varepsilon_{0}=.5 \mathrm{eV}($ Fig. 14b), the exponential characterized by the two points of largest $x$ corresponds to $L_{S} \approx 220 \AA$. The characteristic escape length $L_{S}$ becomes much clearer in a few sample computations carried out for $500 \AA$ films. In such cases it is found that $\mathrm{L}_{\mathrm{S}}$ coincides quite well with the value obtained from the two points of largest $\mathrm{x}$ in $250 \AA$ films. Thus, if one neglects the effects of the sink for electrons at the substrate, one can define escape probabilities of the form

$$
\begin{gathered}
P\left(\varepsilon_{0}, x\right)=P_{0}\left(\xi_{0}\right) e^{-(\tau-x) / L_{S}\left(\xi_{0}\right)} \\
-67-
\end{gathered}
$$




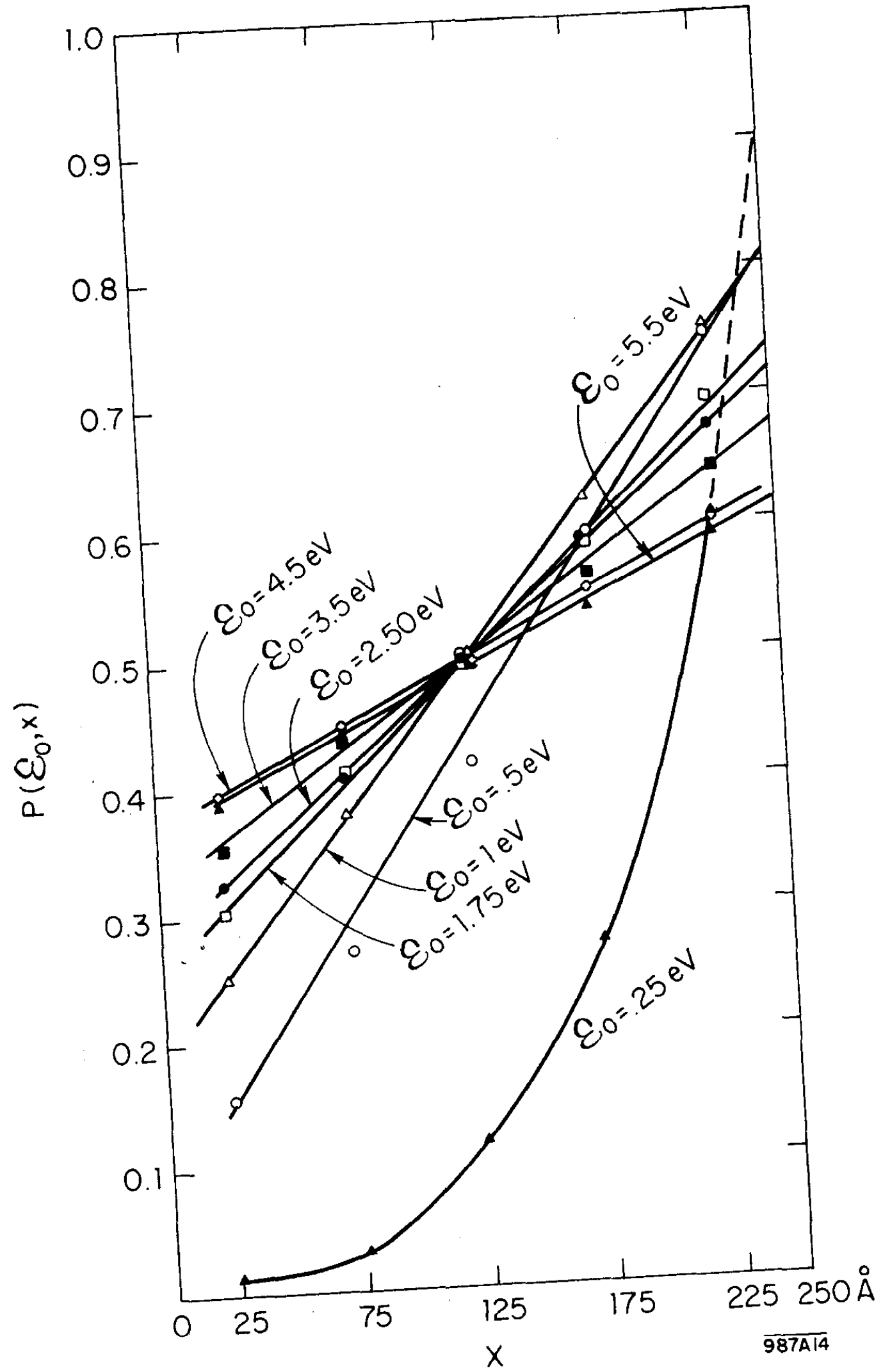
FIG. 13--Probabilities of escape for electrons generated at a position $x$ in a 2
of CsI, with initial energy $\mathscr{E}_{0}$, and an isotropic angular distribution 


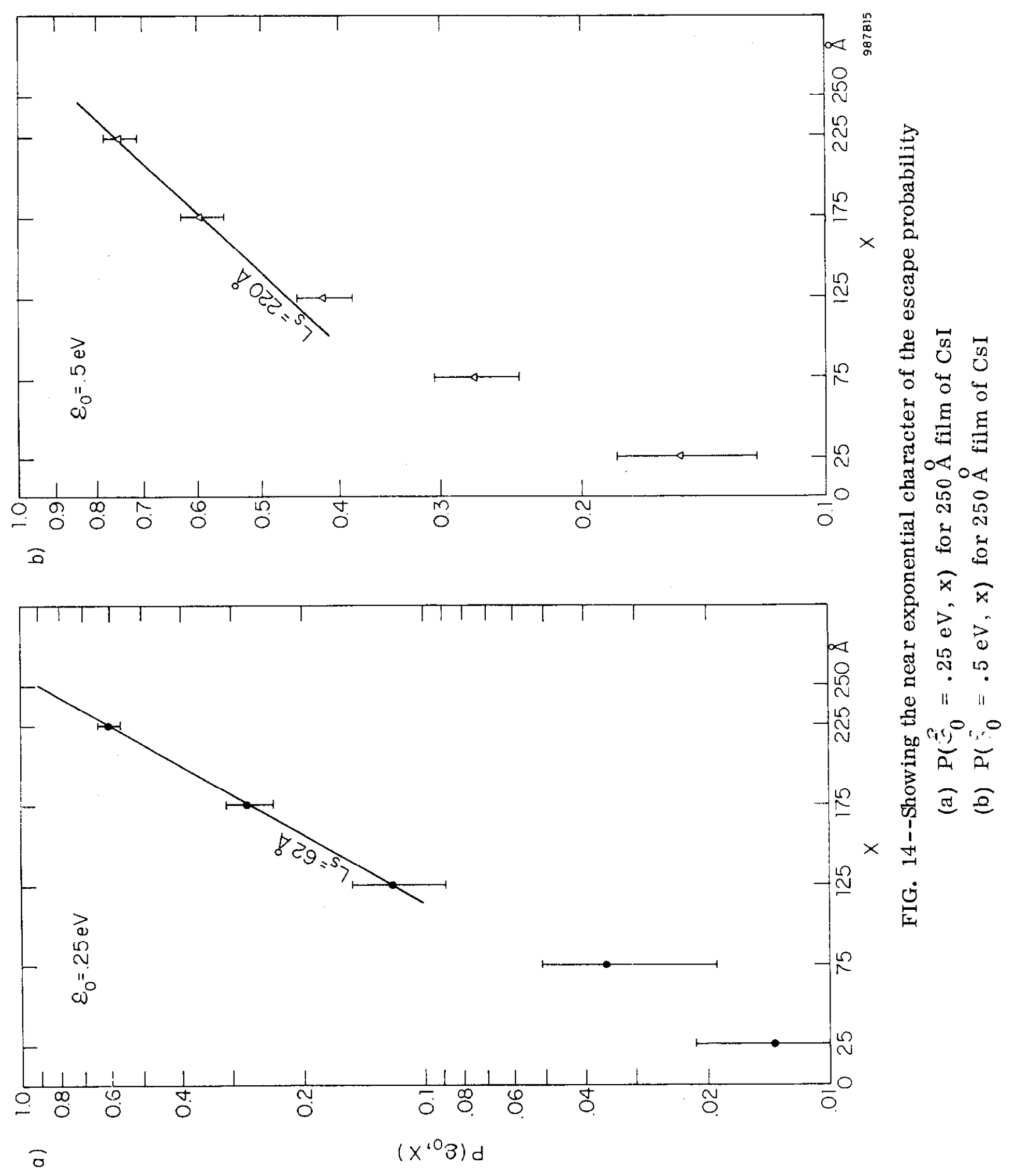


where $\tau$ is the thickness of the film. Table 7 gives values of $\mathrm{P}_{0}\left(\hat{\sigma}_{0}\right)$ and $\mathrm{L}_{\mathrm{S}}$ for CsI and for the three other materials selected.

TABLE 7

\begin{tabular}{|c|c|c|c|c|c|}
\hline \multicolumn{3}{|c|}{$\begin{array}{l}\mathrm{T}=300^{\circ} \mathrm{K}, \mathrm{m}^{*} / \mathrm{m}=1 \\
\mathrm{CsI}, \hbar \omega_{\text {long. }}=.0105 \mathrm{eV}, \mathrm{n}_{\mathrm{q}}=1.987 \\
\mathrm{KCl} \text {, } \hbar \omega_{\text {long. }}=.026 \mathrm{eV}, \mathrm{n}_{\mathrm{q}}=.557\end{array}$} & \multicolumn{3}{|c|}{$\begin{array}{l}\mathrm{NaF}, \hbar \omega_{\text {long. }}=.0513 \mathrm{eV}, \mathrm{n}_{\mathrm{q}}=.159 \\
\mathrm{LiF}, \hbar \omega_{\text {long. }}=.0822 \mathrm{eV}, \mathrm{n}_{\mathrm{q}}=.0435\end{array}$} \\
\hline 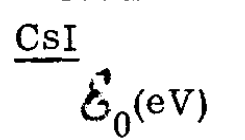 & $P_{0}\left(\varepsilon_{0}\right)$ & $\mathrm{L}_{\mathrm{s}}(\stackrel{\AA}{\mathrm{A}})$ & $\stackrel{\mathrm{KCl}}{E_{0}(\mathrm{eV})}$ & $P_{0}\left(E_{0}\right)$ & $L_{S}(\AA)$ \\
\hline .25 & .9 & 62 & .25 & $\simeq 1.0$ & $<10 \AA$ \\
\hline .5 & .83 & 220 & .5 & .9 & 29 \\
\hline 1.0 & .81 & 260 & 1.0 & .8 & 121 \\
\hline 1.75 & .76 & 295 & 1.75 & .8 & 240 \\
\hline 2.5 & .71 & 375 & 2.5 & .76 & 280 \\
\hline 3.5 & .68 & 395 & 3.5 & .71 & 330 \\
\hline 4.5 & .63 & 540 & 4.5 & .69 & 360 \\
\hline 5.5 & .62 & 650 & 5.5 & .68 & 370 \\
\hline$\stackrel{N a F}{G}_{0}(\mathrm{eV})$ & $\mathrm{P}_{0}\left(\tilde{E}_{0}\right)$ & $\mathrm{L}_{\mathrm{S}}(\AA)$ & $\mathrm{LiF}_{0}(\mathrm{eV})$ & $\mathrm{P}_{0}\left(\mathfrak{E}_{0}\right)$ & $L_{S}(\AA)$ \\
\hline .5 & $\simeq 1$ & $<12 \AA$ & & & \\
\hline 1.0 & .98 & 22 & 1 & $\approx 1$ & $<12 \AA$ \\
\hline 1.75 & .8 & 77 & 1.75 & .9 & 29 \\
\hline 2.5 & .78 & 130 & 2.5 & .86 & 57 \\
\hline 3.5 & .77 & 240 & 3.5 & .75 & 128 \\
\hline 4.5 & .76 & 265 & 4.5 & .75 & 185 \\
\hline 5.5 & .77 & 290 & 5.5 & .76 & 240 \\
\hline 6.5 & .77 & 330 & 6.5 & .75 & 270 \\
\hline 7.5 & .7 & 380 & 7.5 & .76 & 285 \\
\hline
\end{tabular}


The energy distribution of emitted electrons has very characteristic shapes. Figure 15 shows $\mathrm{P}\left(\hat{G}_{\mathrm{i}} ; \hat{G}=1 \mathrm{eV}, \theta=0, \mathrm{x}\right)$, the probability that one electron with initial energy of $1 \mathrm{eV}$, emitted in the forward direction from a source at $\mathrm{x}$, will emerge with energy $\tilde{b}_{\mathrm{i}}$. The analysis of energies has been carried out in channels with a width of $.05 \mathrm{eV}$. The approximate error in each channel is \pm .03 to .05 for $95 \%$ certainty. Normalization of Fig. 15 is to the actual probability of escape. In general, $\mathrm{P}\left(\mathscr{E}_{\mathbf{i}}\right)$ becomes less peaked when $\theta$ departs from zero and when the energy is made lower.

The correlation between the energy and angle of electrons at the exit from the film can be studied approximately by defining a correlation coefficient $X$ as

$$
x=\frac{\sum_{\mathbf{i}}\left(\mathcal{E}_{\mathbf{i}}-\overline{\mathcal{E}}\right)\left(\theta_{\mathbf{i}}-\bar{\theta}\right) \mathrm{P}\left(\mathcal{E}_{\mathbf{i}}, \theta_{\mathbf{i}}\right)}{\sigma_{\mathrm{j}} \sigma_{\theta}}
$$

where the summation is over all outgoing electrons; $P\left(\hat{\sigma}_{i}, \theta_{i}\right)$ is a normalized probability that electrons which started isotropically from a given " $x$ " and energy will reach the surface in a channel corresponding to $\left(\hat{\sigma}_{i}, \theta_{i}\right)$. The means $\bar{\theta}$ and $\bar{\theta}$, and the standard deviations $\sigma_{\hat{6}}$ and $\sigma_{\theta}$ have the customary definitions. It is realized that $X$ calculated in this way only has a definite physical meaning when the distributions are Gaussian. This is not the case here, but $P\left(\hat{\sigma}_{i}\right)$ and $P\left(\theta_{i}\right)$ are well behaved and roughly symmetrical. Therefore $X$ should be a useful measure of correlation.

Data for electrons in a $250 \AA$ film of CsI for a few selected initial energies and positions have been analyzed. The results are given in Table 8 . In order to obtain a better feeling for the meaning of the values of $X$ obtained, the probabilities $P\left(G_{i}, \theta_{i}\right)$ have been plotted in Fig. 16 for the worst case treated, $X=-.08$, for low energy electrons generated far from the exit surface. The plots fail to show any clear correlation, and one can deduce that magnitudes for $X$ of order . 1 indeed represent very little correlation. 


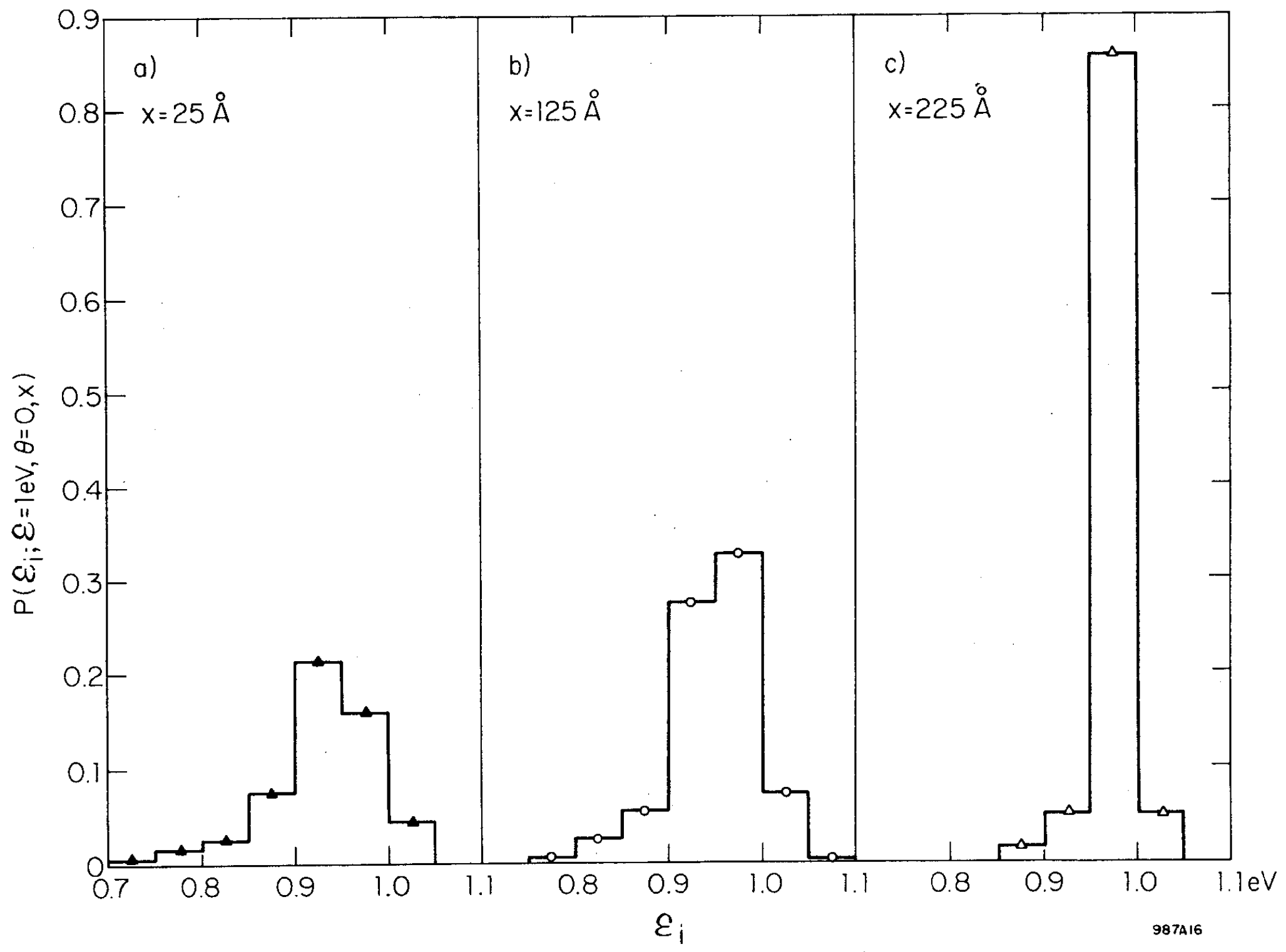

FIG. 15--Exit energy distribution for electrons generated in the forward direction with $\mathcal{E}_{0}=1 \mathrm{eV}$ in a $250 \AA$ film of CsI 


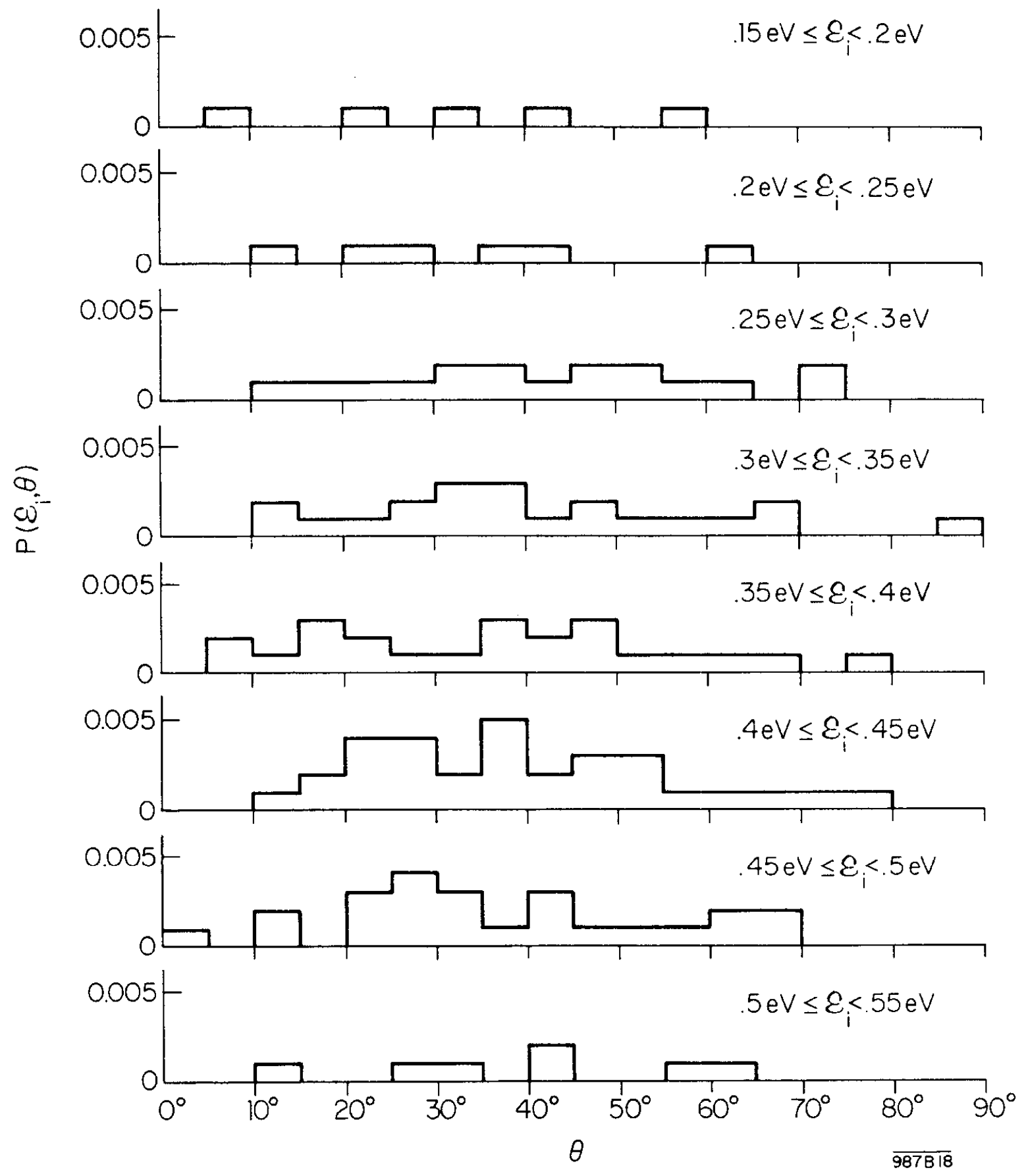

FIG. 16--Probabilities of escape for electrons generated isotropically with $\varepsilon_{0}=.5 \mathrm{eV}$ at $\mathrm{x}=25 \AA$ in a $250 \AA$ film of $\mathrm{CsI}$ 
It should be pointed out that a calculation of escape probabilities based on purely forward scattering, like the one carried out by Khokley and van Vliet, ${ }^{38}$ results in a definite relationship between energy loss and exit angle for the electrons. The inclusion of scattering to other directions has the effect of randomizing the electron distribution and destroying correlation to a very large extent.

\section{TABLE 8}

\begin{tabular}{ccccccc}
$\begin{array}{c}\text { Initial } \\
\text { Position } \\
\mathrm{x}(\mathrm{A})\end{array}$ & $\begin{array}{c}\text { Initial } \\
\text { Energy } \\
(\mathrm{eV})\end{array}$ & $\begin{array}{c}\bar{\delta} \\
(\mathrm{eV})\end{array}$ & $\begin{array}{c}\bar{\theta} \\
(\mathrm{rad})\end{array}$ & $\begin{array}{c}\sigma_{\mathrm{g}} \\
(\mathrm{eV})\end{array}$ & $\begin{array}{c}\sigma_{\theta} \\
(\mathrm{rad})\end{array}$ & $\chi$ \\
\hline 25 & .5 & .368 & .69 & .102 & .378 & -.080 \\
125 & .5 & .39 & .725 & .099 & .319 & -.015 \\
225 & .5 & .442 & .772 & .073 & .323 & +.025 \\
25 & 2.5 & 2.468 & .767 & .041 & .318 & -.029 \\
125 & 2.5 & 2.467 & .792 & .035 & .314 & -.054 \\
225 & 2.5 & 2.470 & .868 & .029 & .304 & +.021 \\
\hline
\end{tabular}

A quantity of interest in studies of electron emission is the mean energy loss suffered by the escaping electrons. The results of the Monte Carlo calculation can be easily analyzed to yield that information. Figure 17a shows the mean energy loss in CsI for electrons of initial energy $\mathscr{E}$ emitted isotropically from a position $\mathrm{x}$. The standard deviation of the energy spectrum, giving a measure of the spread of energies is shown in Fig. 17b. Two details must be pointed out regarding the results: (1) The channel width for the analysis of energy is $.05 \mathrm{eV}$. Therefore, if most of the electrons fall in the channel adjacent to the one corresponding to the initial energy (e.g. , for $\varepsilon_{0}=4.5 \mathrm{eV}$ ), the mean energy loss will be $1 / 2$ channel by design of the computer program. Thus, when the losses are low and the energy distribution becomes narrow, the mean energy loss converges to . $025 \mathrm{eV}$ instead of going towards zero. (2) The 


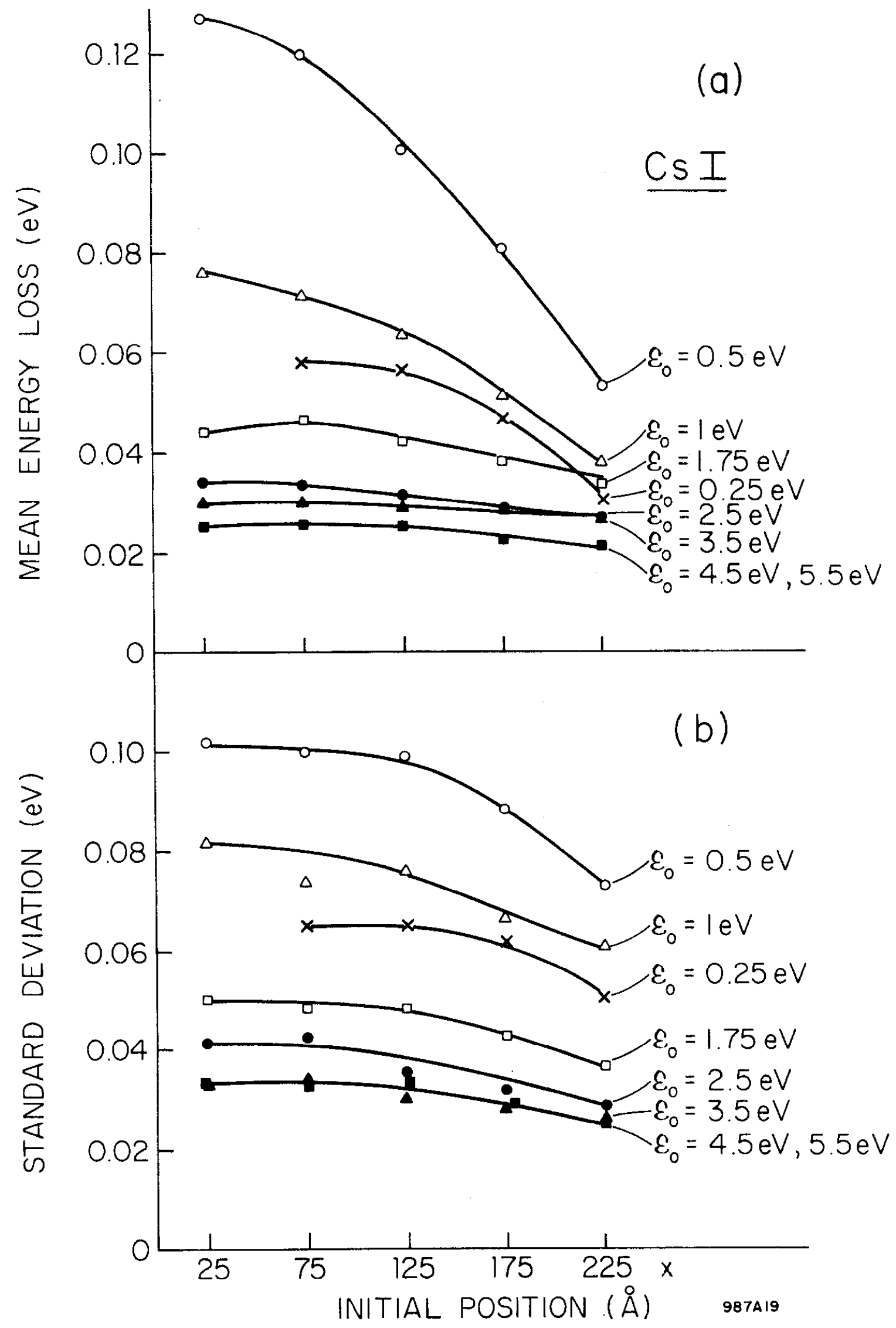

FIG. 17--Mean energy loss (a), and standard deviation (b), for escaping electrons generated isotropically at position $x$ with energy $\sigma_{0}$ in a $250 \mathrm{~A}$ CsI film 
results for $\mathscr{E}^{\circ}=.25 \mathrm{eV}$ seem unduly low. It must be noticed, however, that the only electrons which come out are those which have lost least energy. The rest have been absorbed in the material, resulting in a low probability of escape. The choice of the threshold energy for absorption only affects the results for $G_{0}=.25 \mathrm{eV}$ in CSI in any sensitive manner.

\section{b. Results for $\mathrm{KCl}, \mathrm{NaF}$, and LiF}

Table 7 gives the values of $P_{0}\left(\hat{\theta}_{0}\right)$ and $L_{s}$ for the exponential probabilities of escape of electrons generated isotropically in $\mathrm{KCl}, \mathrm{NaF}$, and $\mathrm{LiF}$, in addition to CsI. The phonon energy to $\omega_{\text {long. }}$ and the average number of phonons per mode $\mathrm{n}_{\mathrm{q}}$ is also shown for $\mathrm{T}=300^{\circ} \mathrm{K}$. The effective mass $\mathrm{m}^{*}$ has been taken equal to the electronic mass $\mathrm{m}$.

As we move from alkali halides with high $\mathrm{Z}$ towards low $\mathrm{Z}$, the phonon energy increases and $n_{q}$ decreases from near 2 to near. 04 . Since the rate of collisions with electron energy gain is proportional to $\mathrm{n}_{\mathrm{q}}$ and the rate with energy loss is proportional to $n_{q}+1$, the relative number of collisions of the second kind becomes very predominant as we go to lighter ions. Since the energy change per collision is increasing, one would definitely expect lower $\mathrm{L}_{\mathrm{S}}$ for a given energy in going from CsI to LiF. This is reflected in the results of the table. Also, the randomization effect due to nonforward collisions becomes very strong for the lighter ions, particularly at low $\xi_{0}$. This is seen by comparing $\mathrm{P}_{0}\left(\hat{\sigma}_{0}\right)$ for a given initial energy between different materials.

It is also expected that the energy losses suffered by excaping electrons will be increasing with lighter ions. Figures 18,19 and 20 show mean energy loss and standard deviation for electrons which were generated isotropically with energy $G_{0}$ at some position $x$. Although the energy losses are of the order of $0.1 \mathrm{eV}$ in a $250 \AA$ film of $\mathrm{CsI}$, they become of the order of $1 \mathrm{eV}$ for NaF, and even larger for $\mathrm{LiF}$. The distribution of energies also broadens as the losses become large. 

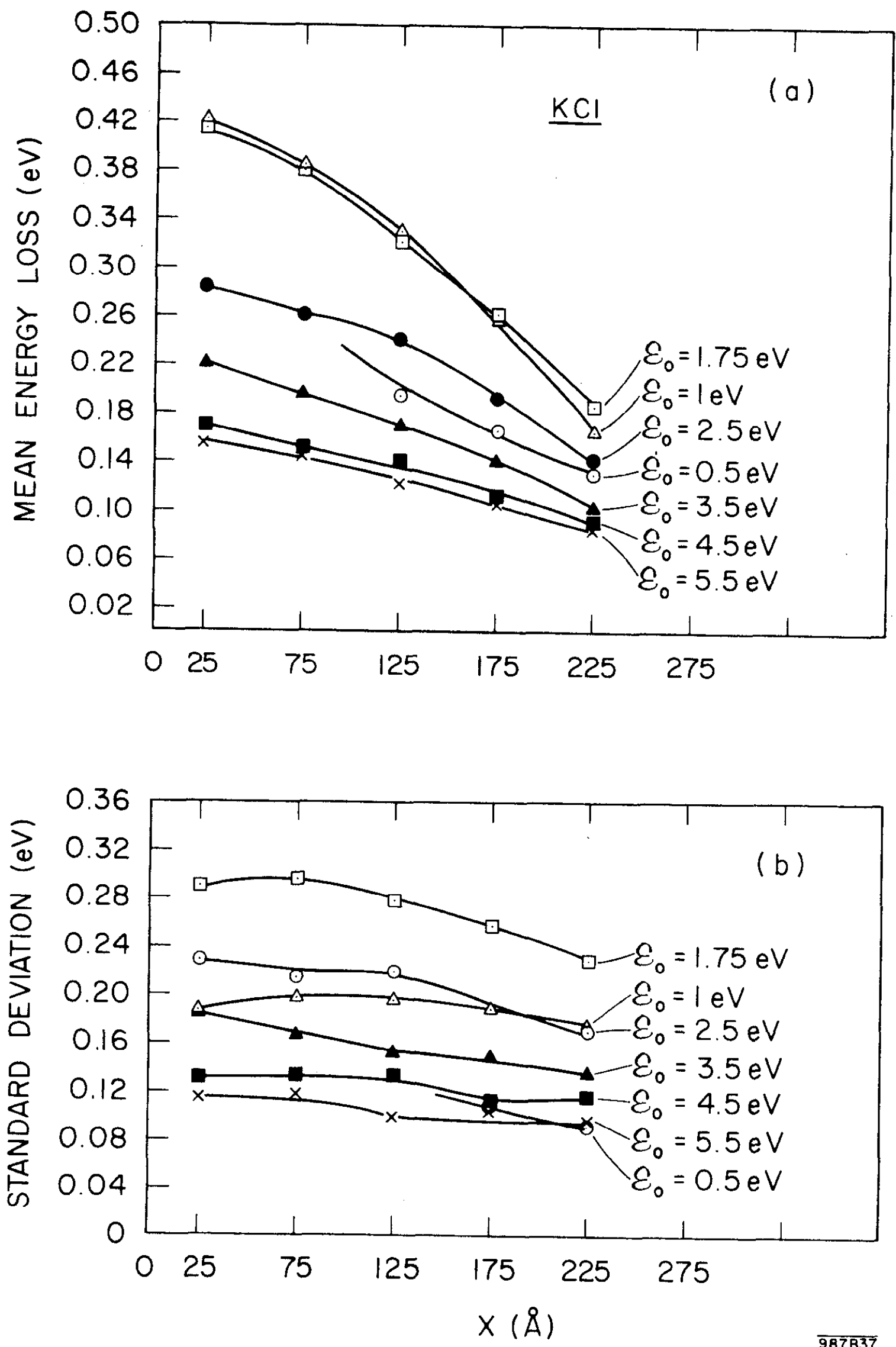

FIG. 18--Mean energy loss (a), and standard deviation (b), for escaping electrons generated isotropically at position $x$ with energy $\sigma_{0}^{2}$ in a $250 \AA \mathrm{KCl}$ film 

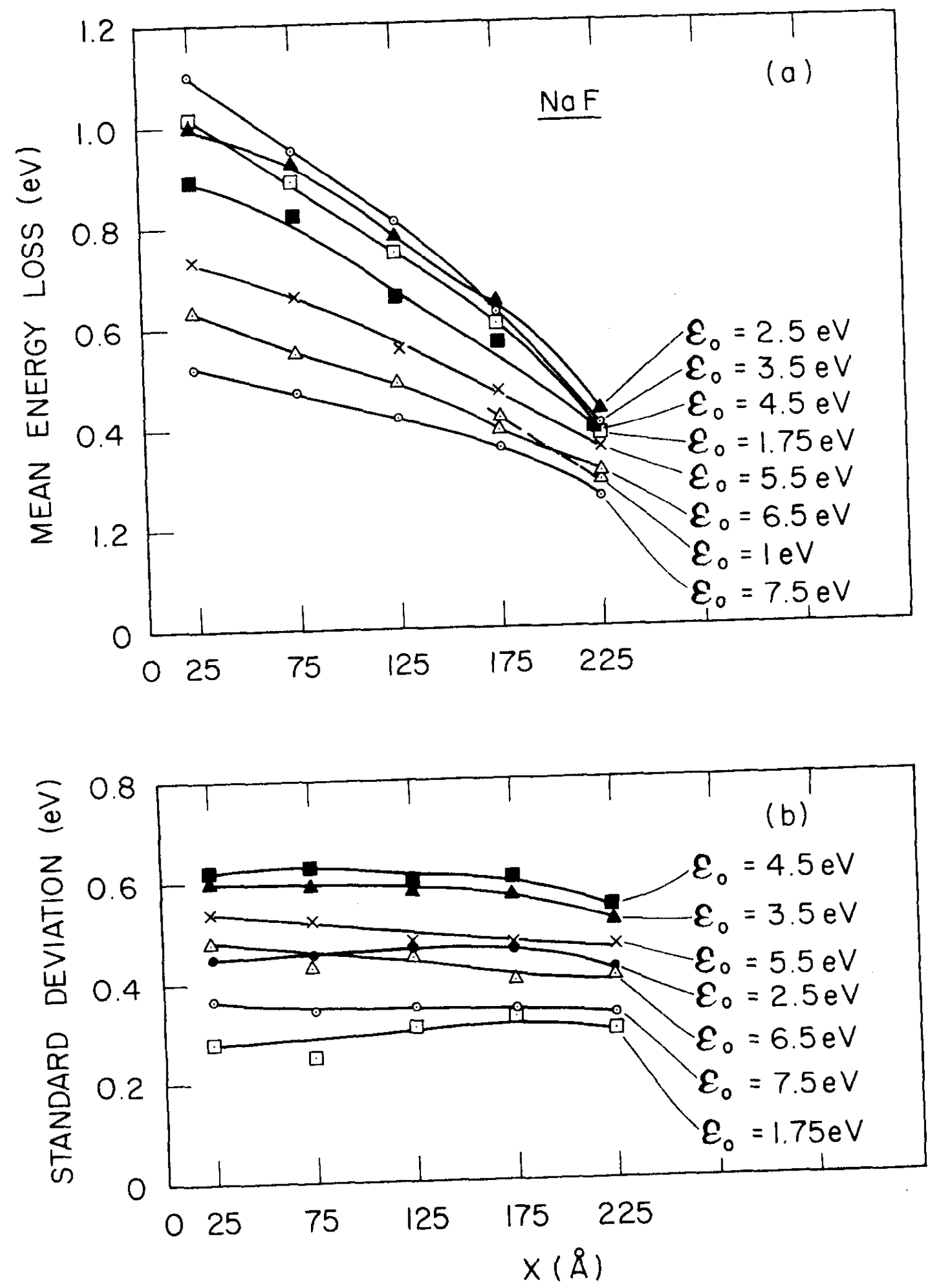

FIG. 19--Mean energy loss (a), and standard deviation (b), for escaping electrons
generated isotropically at position $x$ with energy $\beta_{0}$ in a $250 \mathrm{~A} \mathrm{NaF}$ film 


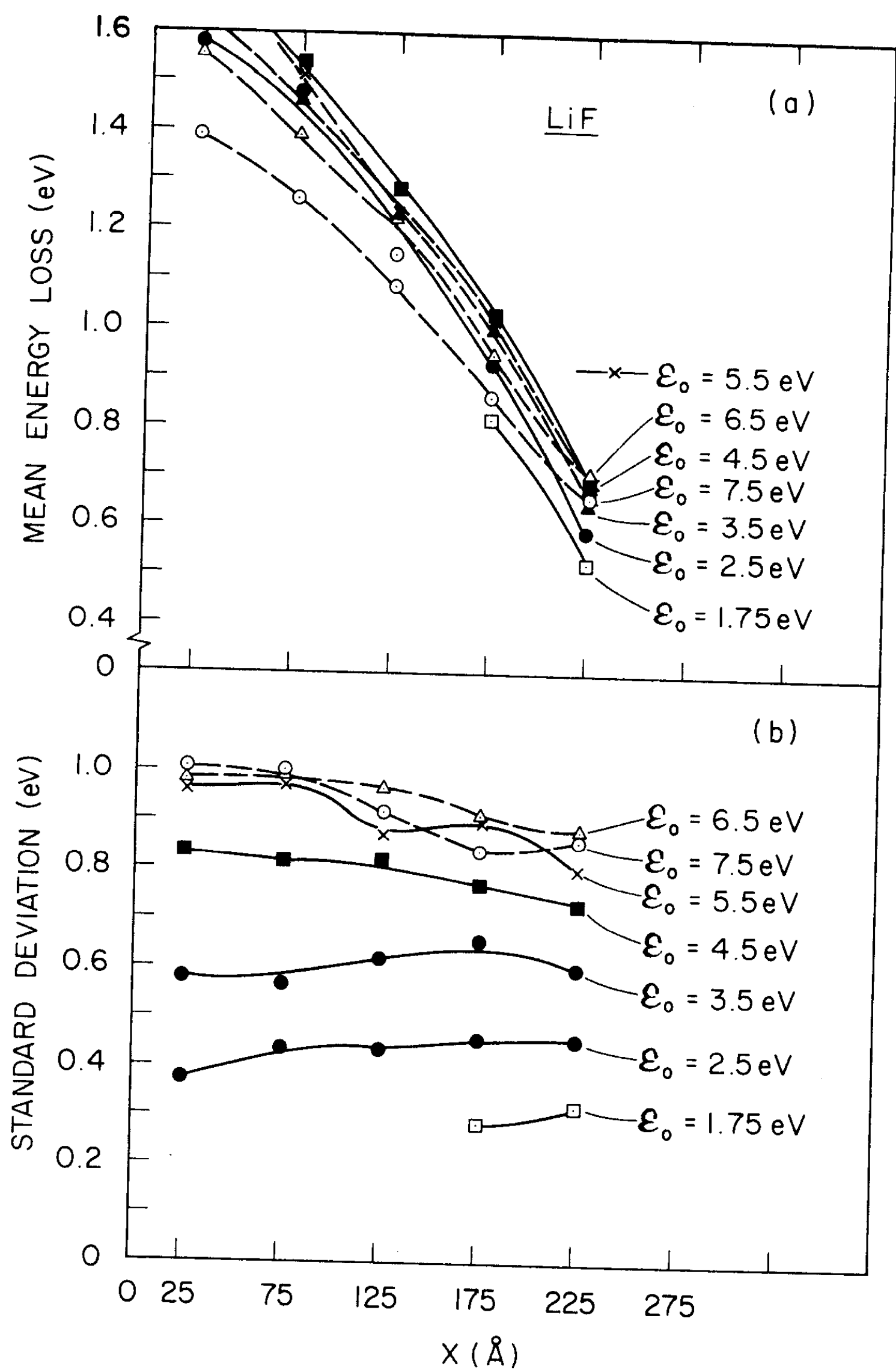

FIG. 20--Mean energy loss (a), and standard deviation (b), for escaping electrons generated isotropically at position $x$ with energy $\mathcal{E}_{0}$ in a $250 \mathrm{~A} \mathrm{LiF}$ film 
A test of angle vs. energy correlation similar to that of Table 8 has been carried out for NaF. Low correlation is still found, although a bit higher than in the case of CsI. The worst result is $X=.12$.

\section{Dependence on Effective Mass and Temperature}

From Eqs. (12) and (14) it is clear that for a given energy $\hat{E}=\hbar^{2} \mathrm{k}^{2} / 2 \mathrm{~m}^{*}$, the scattering rate is proportional to $\left(\mathrm{m}^{*}\right)^{1 / 2}$. Since the velocity is, in turn, proportional to $\left(\mathrm{m}^{*}\right)^{-1 / 2}$, it follows that the mean free path

$$
\langle\lambda\rangle \propto \frac{1}{\mathrm{~m}^{*}} .
$$

How this affects the escape probabilities and energy losses can easily be seen for the case of a film with thickness large compared to the characteristic escape length of the electrons under study. Consider the following Monte Carlo experiment: Take one electron generated in some particular direction at a depth " $d$ " from the exit surface. Take a sequence of random numbers prescribing a trajectory for the electron ending with its escape into the vacuum for $m^{*}=m$. Next consider the same sequence of random numbers for the same inittal conditions but with $m * / m=\beta$. As the experiment progresses, the length of each straight section in the trajectory will be the original length divided by $\beta$. At the end of the sequence the electron will have travelled $1 / \beta$ of its way to the exit surface, or $d / \beta$ towards the vacuum. It follows that its probability of escape and exit energy would be the same as if the electron had $\beta=1$ but had been generated at a depth $\beta$ d.

In the case of temperature dependence, $\langle\lambda\rangle$ is proportional to $1 /\left(2 n_{q}+1\right)$ so that $\beta$ becomes:

$$
\beta=\left(2 n_{q}(T)+1\right) /\left(2 n_{q}\left(300^{\circ} K\right)+1\right)
$$

The correctness of this argument has been tested for CsI with electrons generated with $\theta=0^{\circ}$ and $180^{\circ}$, with energies of $.25 \mathrm{eV}, 1 \mathrm{eV}$, and $2.5 \mathrm{eV}$. The normal 600 to 800 trajectories have been computed for each type of electron. The results 
obtained satisfy the above result perfectly well for $E=.25 \mathrm{eV}$, for electrons generated in both directions at $\mathrm{x} \lesssim 125 \mathrm{~A}$, i. e., in the half film near the exit, and for $\mathscr{O}=1 \mathrm{eV}$ for $\mathrm{x} \lesssim 225 \AA$. As expected, the argument breaks down in higher energies for the $250 \mathrm{~A}$ film ( $\mathrm{L}_{\mathrm{S}}$ too long) or at all the energies when the original location of the electron is too close to the substrate.

One conclusion that can be obtained from the above is the strong dependence of the calculated secondary emission yield on the choice of $\mathrm{m}^{*}$. Indeed, electrons with given initial characteristics will escape with a probability

$$
\mathrm{P}\left(\varepsilon_{0}, \mathrm{~d}\right) \propto \mathrm{e}^{-\mathrm{d} \beta / \mathrm{L}_{\mathrm{s}}\left(\tilde{\sigma}_{0}\right)} \text {. }
$$

It is also possible to obtain an approximation for the temperature dependence of the secondary yield in films of thickness $\tau$. Consider electrons of a single energy, and a uniform generation of secondaries in the film. Then

$$
\frac{\delta\left(300^{\circ} \mathrm{K}\right)}{\delta(\mathrm{T})}=\frac{\int_{\mathrm{x}=0}^{\tau} \mathrm{e}^{-(\tau-\mathrm{x}) / \mathrm{L}_{\mathrm{S}}} \mathrm{dx}}{\int_{\mathrm{x}=0}^{\tau} \mathrm{e}^{-\beta(\tau-\mathrm{x}) / \mathrm{L}_{\mathrm{S}}} \mathrm{dx}}=\beta \frac{\left(1-\mathrm{e}^{-\tau / \mathrm{L}_{\mathrm{S}}}\right)}{\left(1-\mathrm{e}^{-\beta \tau / \mathrm{L}_{\mathrm{S}}}\right)}
$$

and the temperature dependence will be a function of the thickness of the film, as well as of the energy considered.

Since the total yield will be given by integrals (to be converted to summations) over energy and space of the product of a generation function and individual escape probabilities, there does not seem to be any way of writing a simple formulation for $\mathrm{m}^{*}$ and $\tau$ dependence. Once complete calculations are carried out in Chapter III, we shall return to this point. 


\section{Chapter II}

\section{EXPERIMENTS}

\section{A. Preliminary Considerations}

Measurements of secondary emission in reflection have been carried out very extensively in metals, semiconductors and insulators. The review articles $2,3,4,5$ give a comprehensive account of the most important results obtained until 1958 . The work of Jahrreiss ${ }^{24}$ contains an extensive updating to 1964 of reflection and transmission measurements. In 1965, a comprehensive study of reflection secondary emission in all the alkali halides has been reported by Gomoyunova and Letunov, ${ }^{60}$ and in 1966 Edgecumbe and Garwin ${ }^{61,62}$ have reported some measurements in transmission for CsI and $\mathrm{KCl}$. A review of the above-mentioned literature reveals the absence of careful measurements of transmission secondary emission in the alkali halides with a view towards the understanding of their basic properties.

It is well known from the literature that secondary emission from insulators results in charging of the emitter. This charging can have a considerable effect on the secondary emission coefficient $\delta$, as found by Jacobs, ${ }^{63}$ for example, in normal density films of $\mathrm{MgO}$ measured in reflection. For alkali halide films, charging effects are strong in low density deposits, as reported initially by Goetze, Boerio and Green ${ }^{10}$ for $\mathrm{KCl}$. The enhancement of yield observed in such cases has not been reported for normal density films of alkali halides, but no detailed information exists in the literature. Likewise, very little information exists on the energy and angular distribution of emitted secondaries from these sources.

It appears necessary for the purpose of the present investigation to engage in a series of measurements of a selected group of alkali halides with the aim 
of obtaining data with which to relate to the theories of Chapter I. The minimum required outcome of the experiments should be: (1) secondary yield vs. primary energy as a function of film thickness for uncharged films, and (2) energy distribution of secondaries also for uncharged films. A substantial amount of additional information can be obtained from such a series of measurements, but emphasis has been placed on the two main items indicated. It must be pointed out that alkali halide films evaporated in good vacuum have almost identical secondary emission properties in reflection as single crystals cleaved in $10^{-10}$ torr vacuum. ${ }^{60}$ Thus a comparison of the results of single crystal quantum theory with the escape of electrons in evaporated films of alkali halides should be A possible theoretical justification could be that the microcrystals formed in the evaporation contain enough atoms to allow the application of periodic boundary conditions for the determination of normal modes. Also, the apparently low electron affinity of these materials may allow the migration of electrons between microcrystals without any boundary effects.

The requirements for the measurement of secondary yield, $\delta$, in insulators are well known; in order to avoid charging, a pulsed measurement is needed with as few electrons per pulse as possible. The design of the system should also take into consideration the reduction of systematic errors due to scattering and to undesired secondary emission from electrodes in the tube. The measurement of energy distribution in an uncharged film poses more problems, as it cannot be done as fast as that of yield on account of noise problems and there is noway to know a priori how slowly it can be done without charging the insulator film. The most appealing solution has been to build a Kelvin probe to monitor the state of charge of the film as the energy distribution measurements are carried out. Since it has been the experience at the Stanford Linear Accelerator Center that at least $\mathrm{CsI}$ and $\mathrm{KCl}$ films can be moved from one vacuum system to 
another in an atmosphere of dry $\mathrm{N}_{2}$ without any noticeable change in secondary emission properties, the two measuring systems have been built separately, with "in situ" evaporation facilities in only one of them.

\section{B. Measurement of Secondary Yield in Uncharged Films}

1. Description of Equipment

a. Tube Assembly

Figure 21 shows schematically the tube used for the measurements of yield. Elements (1), (2), and (3) comprise the electron gun. This is a very simple Pierce type design, manufactured by Brad Thompson Industries, Inc., Indio ,California, for electron beam welding applications. It consists of a filament (1), which has been made of .010-inch tungsten wire into a hairpin configuration for the present application, a focusing and control electrode (2), and an anode (3) with an opening of 5/16-inch diameter operating at ground potential. The filament is supplied from a floating d.c. power supply with up to 15 amps of moderately well-filtered current (.25 percent RMS ripple). The control electrode is supplied by a floating voltage source of up to $-1000 \mathrm{~V} . \mathrm{D} . \mathrm{C}$. with respect to the filament. The accelerating potential is then applied to the filament and the current and focusing are controlled by filament temperature and control electrode-to-filament potential.

The electrons leaving the anode opening find a 3-plate collimator (4). The first plate contains an insert of thickness . 010-inch and aperture 1/16-inch defining the beam size. The small thickness helps reduce the number of electrons which will be scattered at a shallow angle by the edges of the collimator. Such electrons would tend to reduce the monochromatic quality of the beam. Subsequent openings are progressively larger so that electrons passing through the first opening can reach any point in the dynode (1-inch dia.) without collisions. The function of the second and third collimators is, then, to remove electrons scattered by the first 


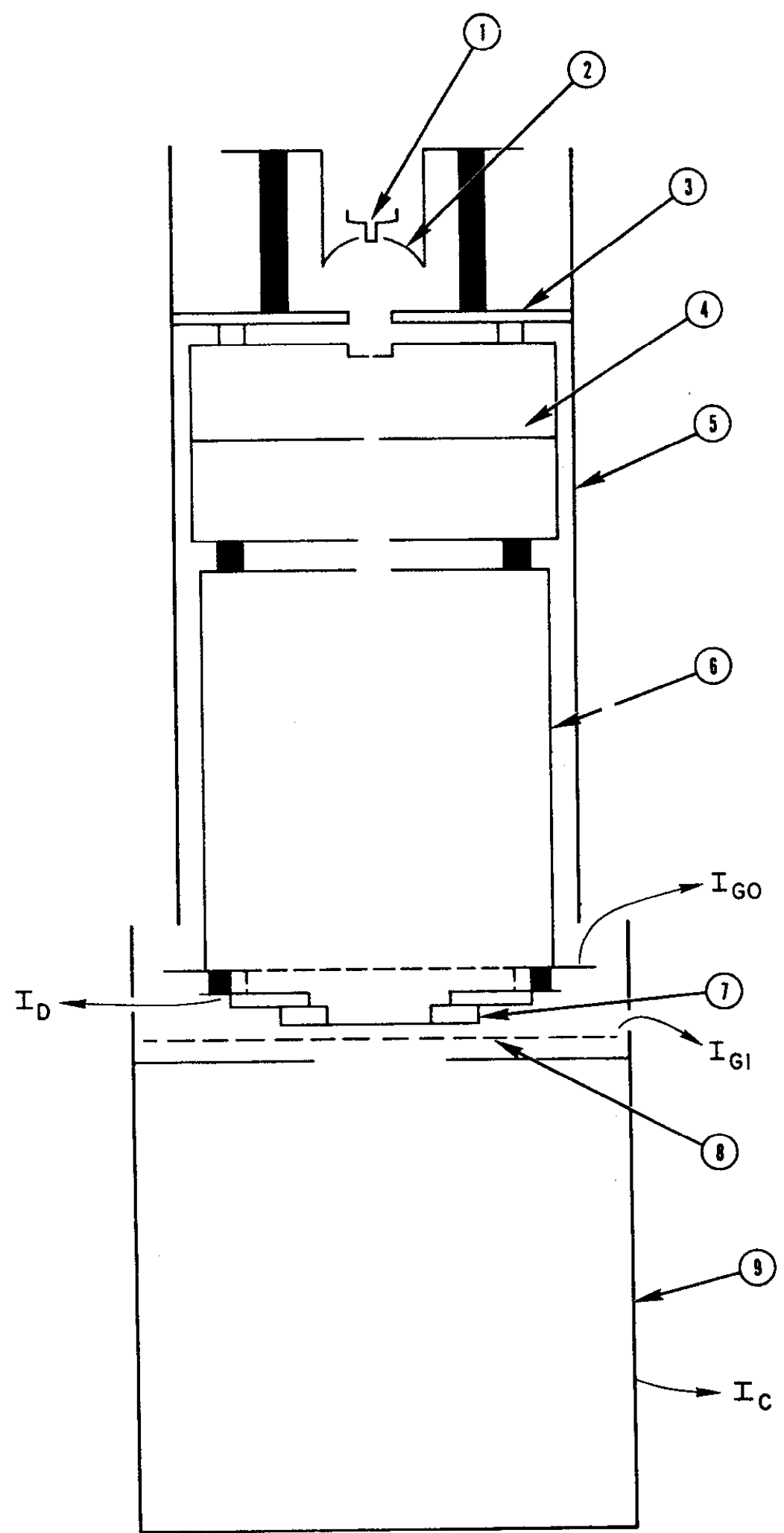

APPROX. SCALE $\stackrel{1 "}{\longmapsto}$

- ceramic insulators

FIG. 21--Schematic of the tube used for measurement of secondary yield 
one at angles other than very shallow. All the drift space in the tube is surrounded by a nickel shroud (5) as protection against magnetic fields. The primary beam then penetrates a fully enclosed system, consisting of four electrodes, through an aperture of 5/8-inch diameter on top of electrode (5). This electrode, which operates at - 90 volts, consists of a grid for the suppression of secondary emission from the entrance side of the dynode (7) and a Faraday cup for the collection of electrons backscattered from the dynode. No attempt has been made to eliminate secondary emission from the walls of the cup due to backscattered primaries because it does not affect the measurements, as will be shown below. This suppressor electrode will be termed $G_{0}$. The dynode (7) consists of an $A 1$ support ring of 2-inch diameter and approximately $1 / 8$-inch thickness with an opening of 1-inch diameter. A $1000 \AA$ film of $\mathrm{Al}_{2} \mathrm{O}_{3}$ obtained by anodic oxidation and etching of very pure $A 1$ foil ${ }^{64}$ is glued to the support, and $500 \AA$ of $A l$ are evaporated onto it to supply electrical contact to the alkali halide. ${ }^{65}$ The dynode is easily mounted onto its holder in the tube. The dynode will be designated by D. It is operated at ground potential.

The collector assembly, part of which can be made to swing out under vacuum to allow the evaporation of the salts, is formed by a collector (9), designated $C$, and a suppressor grid (8) designated $G_{1}$. The collector assembly is again a Faraday cup with an aperture of 1-3/4-inch diameter placed at a distance of $3 / 8$ inch from the dynode, designed to prevent the majority of high energy electrons backscattered by the collector from reaching the dynode. The grid $G_{1}$ will suppress secondary emis sion from the collector and/or from the dynode depending on the measurement carried out. There are two standard measurements to be performed normally:

(1) Measurement of transmission yield, $\delta_{t}$, in which one is interested in finding the total number of electrons leaving the dynode, regardless of energy. For this case, the operating voltages are $V_{G_{1}}=+45 \mathrm{~V}, V_{C}=+90 \mathrm{~V}$. Low energy 
electrons (secondaries) emitted by the dynode are attracted towards the collector and intercepted by $G_{1}$ (very few of them) or by the collector, either inside the cage passing through the 1-3/4-inch opening or at the diaphragm forming the cup enclosure. Higher energy electrons transmitted through the film come out at average angles that depend on energy and thickness, but the location of the diaphragm and size of opening insures that they go mostly into the cage. Energetic backscattered electrons can only leave the cage through the opening and this presents a moderately small solid angle from any point inside it. Secondaries generated inside the cage cannot come out due to the potential at $G_{1}$. Transmitted primaries which do not enter the cage can be backscattered, resulting in erroneous results. The estimated errors will be analyzed below. The placement of the diaphragm as close as possible to the dynode appears to be the best solution to both letting as many transmitted primaries into the cage and as few as possible out of it.

(2) Measurement of transmission coefficient, $\eta_{t}$, in which one wants to suppress secondary emission from the dynode, and measure only the energetic electrons transmitted. Operating voltages are $\mathrm{V}_{\mathrm{G}_{1}}=-45 \mathrm{~V}, \mathrm{~V}_{\mathrm{C}}=0 \mathrm{~V}$. The discussion regarding backscattering effects given above applies here as well. The only difference is that a negative $V_{G_{1}}$ will suppress secondaries from the dynode and secondaries from the grid $G_{1}$ will be collected at the dynode.

The anode (3), the collimators, and the cage (6) are made of stainless steel. The collector and its diaphragm are of aluminum, this choice having been made on account of its low backscattering coefficient. The grids are $97 \%$ open $\mathrm{Ni}, 20$ lines per inch. Ceramic insulators are used throughout.

With the collector assembly moved out of position, the dynode area is exposed to a boat directly underneath in which premelted halide salts are ready for evapora tion. The dynode to boat distance is approximately 7 inches. At the same distance from the boat, but angled about $30^{\circ}$, the head of a water-cooled quartz crystal evaporation monitor ${ }^{66}$ has been placed. It has been made sure that the crystal location is 
in full line-of-sight of the whole surface of the boat. This method of evaporation mass control has repeatedly been found accurate within a few percent in calibra tions carried out at SLAC.

The system is enclosed in a Viton sealed glass belljar vacuum system. Initial rough pumping to about 5 -inch $\mathrm{Hg}$ is provided by a Varian venturi-type pump driven by oil-free compressed air. Two Varian Vac-Sorb pumps continue the rough pumping up to $5 \mu \mathrm{Hg}$, at which point a $400 \mathrm{l} / \mathrm{sec}$ ion pump takes over. With the system vented exclusively to dry $N_{2}$, it is possible to cycle to about $5 \times 10^{-7}$ torr several times a day without warming up the roughing pumps and obtain the best pressure of $2 \times 10^{-8}$ torr for the system in about 6 hours.

\section{b. Electronic Circuits}

Figure 22 shows the electronic circuits for the dc and pulsed measurements. Bias for the four electrodes is supplied by well-insulated, floating batteries through $8.2 \mathrm{M} \Omega$ resistors. For dc measurements, the currents $I_{C}$ and $\mathrm{I}_{\mathrm{G}_{1}}$ are added and measured by a floating battery-operated Keithley 601 electrometer. This total current, $I_{C+G_{1}}$, is then added to $I_{G_{0}}+I_{D}$ and measured by a second electrometer as the primary current. The transmission gain $\delta_{t}$, transmission coefficient $\eta_{t}$, and gain $\delta$ are then given by

$$
\begin{array}{lll}
\delta_{t}=\frac{-I_{C+G_{1}}}{.97 I_{p}} & \text { for } & { }_{V_{C}}=+90 \mathrm{~V} \\
V_{G_{1}}=+45 V \\
\eta_{t}=\frac{-I_{C+G_{1}}}{.97 I_{p}} & \text { for } \quad V_{C}=0 \mathrm{~V} \\
V_{G_{1}}=-45 V
\end{array}
$$




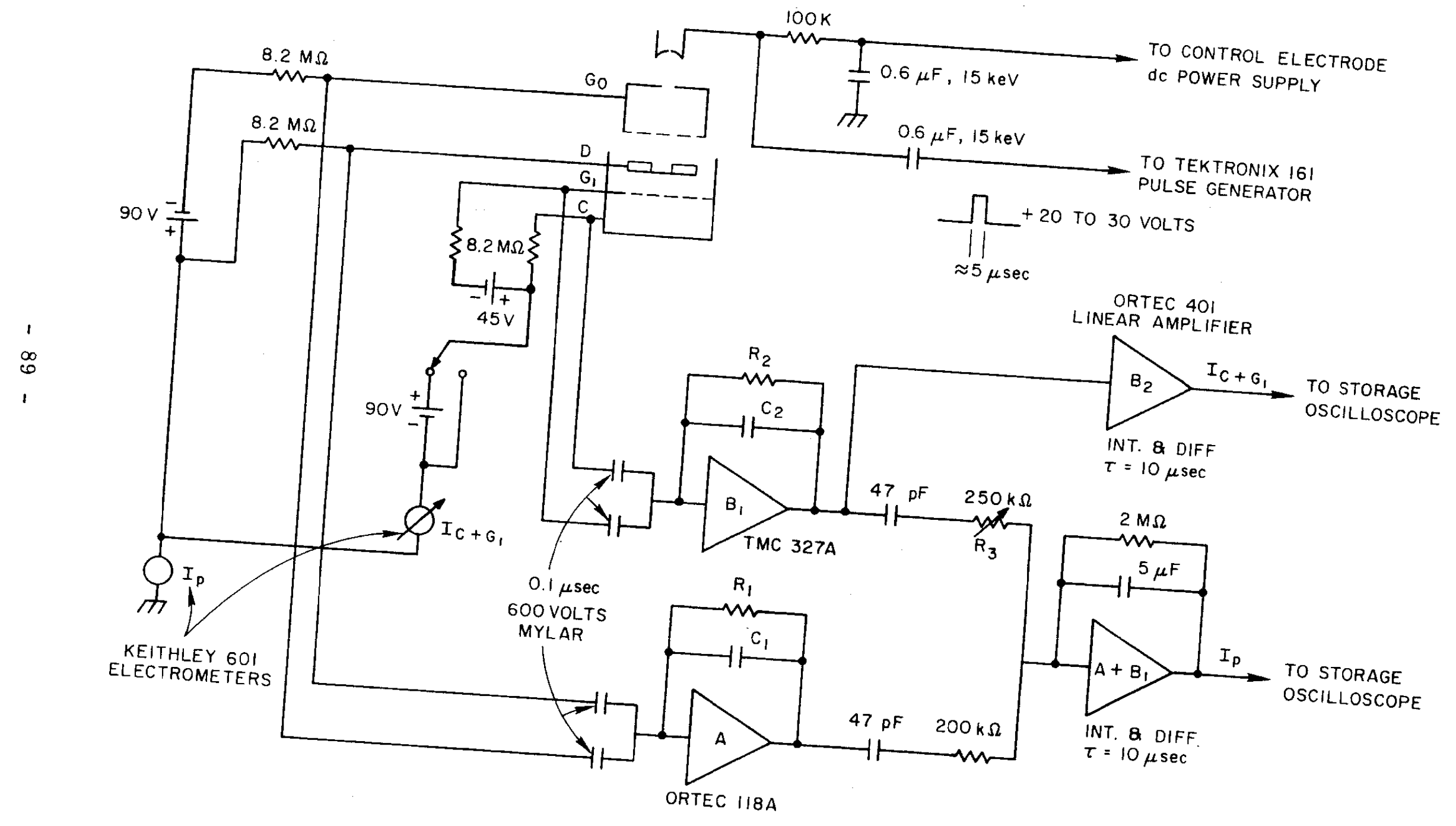

FET CHARGE

$\overline{987834}$

SENSITIVE AMPLIFIERS

FIG. 22--Schematic of circuits used for measurement of secondary yield 
Expressions (17) to (19) will be the definitions for secondary emission characteristics used throughout the present work. The factor of .97 multiplying $I_{p}$ takes into account the fact that approximately $3 \%$ of primaries are intercepted by the suppressor grid $G_{0}$. The collimator prevents interception of electrons by the $G_{0}$ cup.

The convention for the sign of the currents, to be used consistently in this report, will be the following:

The assembly $\left(G_{0}, D, G_{1}, C\right)$ is considered as a "black box" with 4 wires and one hole. Conventional current coming out of the box is positive, as would be measured by a meter. Thus

$$
I_{G_{0}}+I_{D}+I_{G_{1}}+I_{C}=-I_{p} \text {, with } I_{p}>0
$$

The current passing through a wire connected to an element with a net collection of electrons will be negative.

The above definition of $I_{p}$ implies that the amount of charge stored inside the "black box" per unit time is small compared to the true primary current. As shown by some approximate estimates of positive charge trapped in photoemission in $\mathrm{CsI}^{48}$ and from preliminary measurements of film surface potential on the same material at SLAC it appears that about one hole is trapped without recombining for 1000 electrons leaving the alkali halide. This effect will be considered negligible and extendible to other alkali halides in the absence of any anomalous experimental indications.

For pulsed measurements, the control electrode of the electron gun is biased beyond cutoff. A single pulse of approximately $5 \mu \mathrm{sec}$ duration and sufficient amplitude to turn the beam on (usually +30 to 40 volts) is applied to the control electrode from a Tektronix 161 pulse generator. By carefully controlling filament current and the acceleration potential, it is found possible to predetermine the setting of the dc control electrode voltage in such a way to insure no acsidental turning on of the beam or high current pulses. 
Since the aim of this pulsed measurement is to obtain the best signal-tonoise ratio with a reasonable amount of effort, it has been decided to borrow from the very well-developed technology of electronics for semiconductor nuclear spectrometry. Its aim is also that of the accurate measurement of very small charge pulses. The bibliography of that field is quite extensive and only a few basic works will be indicated here: Gillespie, ${ }^{67}$ Fairstein, ${ }^{68}$ and Chase et al,${ }^{69}$ discuss the essential characteristics of low-noise amplifiers to be used for the measurement of current pulses from a predominantly capacitive high impedance source. It is shown that a charge-sensitive input amplifier provides the best gain stability quite independent of small changes in input capacitance. The input capacitance has a very important bearing on the obtainable $\mathrm{S} / \mathrm{N}$ ratio; for all practical purposes $\mathrm{N} \propto \mathrm{C}_{\text {in }}$. It is therefore advisable to reduce the wiring capacitance of the amplifier input to a minimum. After the charge pulse has been integrated, the best simple treatment of the voltage step obtained is an integration and differentiation with equal time constant $T$, which should be as short as possible, although it should be longer than the duration of the charge pulse into the input amplifier. Radeka ${ }^{70}$ examines the behavior of field effect transistors (FET) in detail, and finds that the inherent low gate leakage current makes them very attractive as input elements in the charge integrators. The importance of the transconductance of the FET is pointed out as $N \propto 1 / g_{m}$ for a fixed input and gate-to-source capacitance. At present, FET's with $\mathrm{g}_{\mathrm{m}}$ comparable to the best low-noise pentodes are available and even at $C_{\text {in }}$ up to $50 \mathrm{pfd}$, FET preamplifiers are of advantage. At $C_{\text {in }}=100 \mathrm{pfd}$, $\mathrm{S} / \mathrm{N}$ is approximately $25 \%$ worse with presently available commercial single FET amplifiers as compared to pentode inputs. FET's can be paralleled for a substantial improvement at high $C_{\text {in }}$. The capacitance seen by each amplifier 
of Fig. 20 is very close to $100 \mathrm{pfd}$, due to the large physical size of the electrodes, plus feedthrough, wiring and other stray capacitance. Clearly, the amplifiers are not being used to the best advantage, but it will be shown that there is no need to refine the measurement technique any further.

The amplifier $A$ integrates the pulsed $I_{G_{0}+D}$ with a long time constant $\mathrm{R}_{1} \mathrm{C}_{1}$. A subsequent second amplification loop (not shown in Fig. 20) contains a pole-zero cancellation network and a single differentation which has been adjusted to provide a single decay time constant of $50 \mu \mathrm{sec}$. This is equal to the predominant time constant of amplifier $B_{1}$, determined by $R_{2} C_{2}$, which integrates $I_{C+G_{1}}$. The output signal of $B_{1}$ is taken to a linear amplifier $B_{2}$ with variable gain and an adjustable integrator and differentiator. The time constants $\tau$ are set to $10 \mu \mathrm{sec}$, the longest available in the amplifier. Results with signals from $B_{1}$ with a risetime of $5 \mu \mathrm{sec}$ are good. The output of the second amplifier $B_{2}$ is $I_{C+G_{1}}$ ready to be displayed in a Tektronix storage oscilloscope. Its waveform is like $\mathrm{t} \mathrm{e}^{-\mathrm{t} / \tau}$ with a maximum at $\mathrm{t} \approx \tau$. This would be exact for a perfect step into $B_{2}$.

The signals from $A$ and $B_{1}$ are added and likewise integrated and differentiated with $\tau=10 \mu \mathrm{sec}$ by a simple three-stage operational amplifier $\left(A+B_{1}\right)$. The coupling between $B_{1}$ and $\left(A+B_{1}\right)$ contains a variable resistor for small adjustments of the relative gain of channels $A$ and $B_{1}$. The output $\left(A+B_{1}\right)$ corresponds to $I_{p}$, ready to be displayed simultaneously with $I_{C+G_{1}}$ in a storage oscilloscope (chopped mode). 


\section{Testing of Equipment}

a. Evaluation of the Tube Performance and Systematic Errors

A series of evaluation tests have been conducted with the measuring system in order to determine its behavior and the approximate size of systematic errors. First, the characteristics of the electron beam have been studied. By placing a solid glass plate covered with a $100 \AA$ layer of $\mathrm{Ag}$ and a thin coating of $\mathrm{ZnS}$ in the dynode holder, an image of the electron beam could be obtained. The shadow of grid $G_{0}$ in the image was used to determine the approximate spot size. At a primary current $I_{p}$ of $10 \mathrm{nA}$ with the beam well focused, the area of the spot was approximately $5 \mathrm{~mm}^{2}$ at the desired operating energies of 4 to $12 \mathrm{keV}$. The spot was located slightly off-center in the dynode opening, due to the filament being also slightly off-center. At $\mathrm{I}_{\mathrm{p}}=2 \mathrm{nA}$, the beam area was only a little smaller. At $I_{p}=10 \mathrm{nA}$ again, but with a defocused beam, the image was still contained within the 1-inch dynode diameter. A convenient filament current was chosen $(\simeq 12.5 \mathrm{Amp}$ ) and the setting of control electrode potentials was established for the energies of interest. They correspond to the well-focused beam of characteristics indicated above.

A retarding potential measurement of the beam current was carried at $\mathrm{E}_{\mathrm{p}} \simeq 2 \mathrm{keV}$. Higher accelerating potentials could not be used because the insulation at the four tube electrodes was not designed to withstand high voltages. With an empty dynode holder, measurements were done in two ways:

(1) $G_{0}+D$ were used as retarding elements, while the current was collected at $\mathrm{C}+\mathrm{G}_{1} \cdot \mathrm{V}_{\mathrm{C}}$ was 0 volts, $\mathrm{V}_{\mathrm{G}_{1}}=-45$ volts. In this case, letting $\mathrm{V}_{0}$ be the magnitude of retarding potential for $I_{G_{0}+D}=I_{C+G_{1}}=0$, it was found that at $\mathrm{V}_{0}-140$ volts some current was starting to be switched from $\mathrm{C}+\mathrm{G}_{1}$ to $\mathrm{G}_{0}+\mathrm{D}$ keeping the sum constant. At $V_{0}-6$ volts each of the two electrode groups was receiving half the current. Then within the 6 volt interval the two currents became 
zero. This switching effect has been attributed to a lens effect at the high field spacing between the third collimator and the top of the $G_{0}$ cup.

(2) $G_{0}+D$ at ground potential, retarding potential applied to $C$, with $\mathrm{V}_{\mathrm{G}_{1}}=\mathrm{V}_{\mathrm{C}}-45$ volts. In this case, at $\mathrm{V}_{0}-30$ volts, current started being switched from the collector assembly to the $\mathrm{G}_{\mathrm{O}}+\mathrm{D}$ group. At $\mathrm{V}_{0}, \mathrm{I}_{\mathrm{C}+\mathrm{G}_{1}}$ went to zero, and all the beam current was retained and collected at the $G_{0}$ group. The retarding field is applied between the two $97 \%$ open grids and a certain amount of broadening of the cutoff can be expected.

The conclusion is that the electron beam is essentially monochromatic and that the beam collimator functions properly.

The behavior of the $\left(G_{0}+D\right)$ part of the assembly has been studied by measuring the backscattering coefficients of thick $(.010 \mathrm{inch})$ foils of $\mathrm{Al}$ and Ta mounted at the dynode support. The approximate backscattering coefficients $\eta$ of these two elements are .15 and .43 , respectively. $8,30,31$ Since no effort has been made to prevent secondary emission from the $G_{0}$ cup, the measurements of $\mathrm{I}_{\mathrm{G}_{0}}$ will not give a direct measure of backscattering. In fact, the values of $I_{G_{0}} / I_{p}$ obtained with $V_{D}=0, V_{G_{0}}=-90$ volts, shown in Table 9 , indicate a very strong secondary emission effect, very dependent on $\mathrm{E}_{\mathrm{p}}$. If secondary emission were completely eliminated, normalized values of $\mathrm{I}_{\mathrm{G}_{0}}$ of approximately -.15 and -.43 should be obtained.

Table 9

\begin{tabular}{|c|c|c|}
\hline $\mathrm{E}_{\mathrm{p}}$ & Normalized & $\mathrm{I}_{\mathrm{G}_{0}}$ \\
\cline { 2 - 3 } $\mathrm{keV}$ & $\mathrm{Al}$ & $\mathrm{Ta}$ \\
\hline 4 & -.0267 & -.07 \\
6 & -.0546 & -.134 \\
8 & -.073 & -.18 \\
10 & -.085 & -.212 \\
11 & -.095 & -.27 \\
\hline
\end{tabular}


It is possible to investigate this discrepancy by sweeping $V_{D}$ around -90 volts in order to establish the value of the current due to secondary electrons leaving the $\mathrm{G}_{0}$ cup. Figure 23 shows a plot of normalized $\mathrm{I}_{\mathrm{D}} \mathrm{vs} \mathrm{V}_{\mathrm{D}}$ for a Ta foil, at $\mathrm{E}_{\mathrm{p}}=4$ and $12 \mathrm{keV}$. The point of true zero cpd can be established approximately by realizing that the work function of $\mathrm{Ni}$ (the $\mathrm{G}_{0}$ grid material) is higher than that of Ta by roughly . 5 volts (from standard handbook references), so that at $V_{D} \simeq-90.5$ volts, a true zero $\mathrm{cpd}$ can be considered to exist. Also, from arguments to be presented carefully later in this chapter, for a retarding potential measurement with planar geometry, and an angular distribution of electrons which is roughly like a cosine, the true zero occurs at an inflection point in the $\mathrm{I}$ vs. V characteristics. Again this corresponds approximately to $\mathrm{V}_{\mathrm{D}}=-90.5$ volts. The difference in current between this point and asymptote at large negative $V_{D}$ is due to secondary emission from the $\mathrm{G}_{0}$ grid and cup. Adding these currents to the results of normalized $\mathrm{I}_{\mathrm{G}_{0}}$ of Table 9 , it is found that $\eta \simeq .35$ at $\mathrm{E}_{\mathrm{p}}=4 \mathrm{keV}$ and $\eta \simeq .46$ at $\mathrm{E}_{\mathrm{p}}=12 \mathrm{keV}$. The latter value is the more reliable one as the lower slope of the $I_{D}$ vs. $V_{D}$ characteristic makes the result less sensitive to the accurate position of the true zero $\mathrm{cpd}$. In spite of the fact that the surface condition of the materials is not well defined, these results seem to indicate clearly that the deficiency in $\mathrm{I}_{\mathrm{G}_{0}}$ noted in Table 9 is indeed due to secondary emission from the $\mathrm{G}_{0}$ cup. This emission has no direct effect on the determination of $\mathrm{I}_{\mathrm{p}}$ or $\mathrm{I}_{\mathrm{C}+\mathrm{G}_{1}}$ needed for the yield measurements, except on account of the following two possible leaks for secondaries:

(1) Leakage through the top aperture of the $G_{0}$ cup. By making a retarding potential measurement between the whole $\left(G_{0}+D\right)$ assembly and ground, it is found that the normalized leakage is less than .01, i.e., the value of $I_{p}$ obtained by the sum of electrode currents is too small by $1 \%$ at worse, due to this particular effect. 


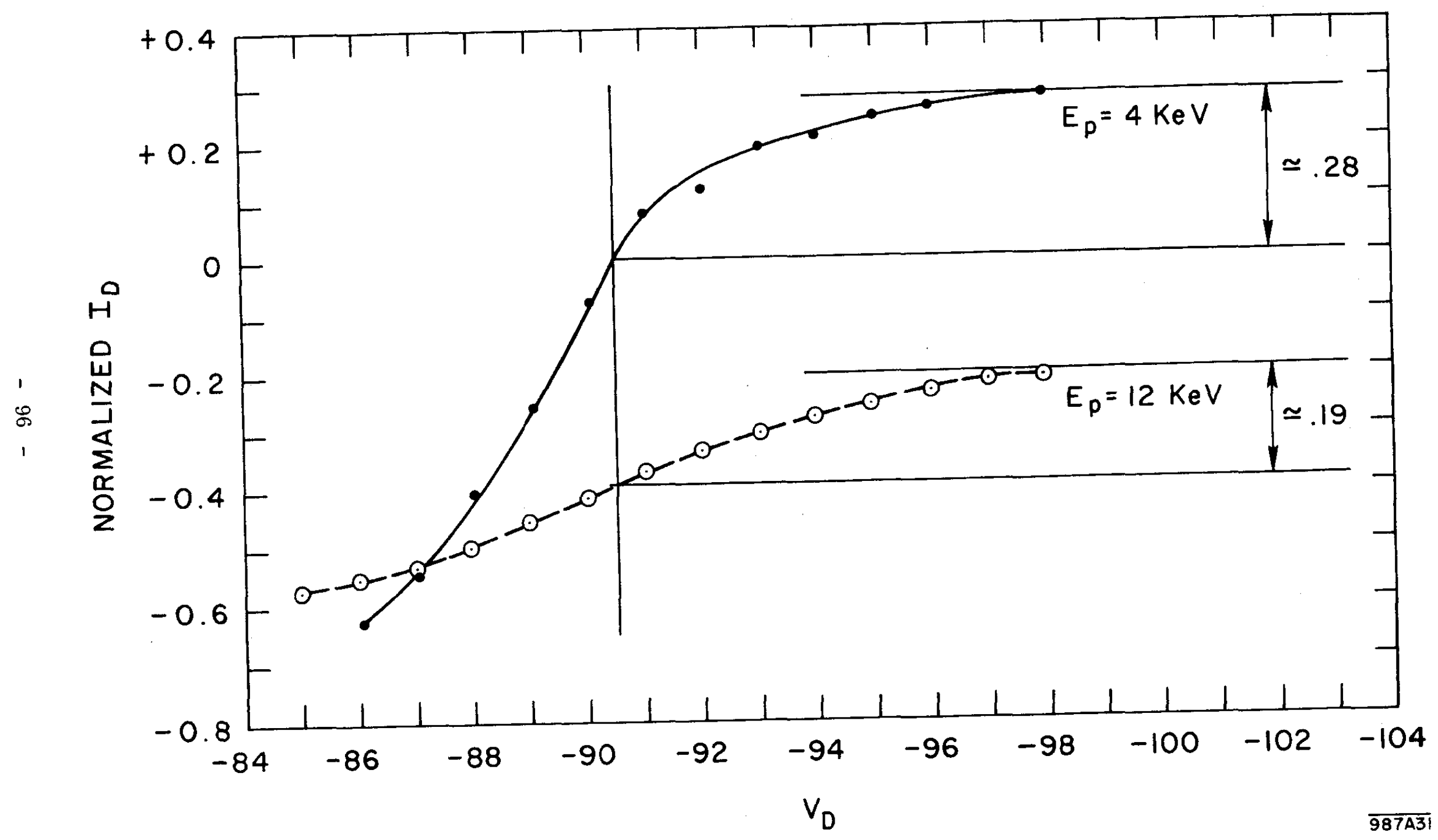

FIG. 23--Retarding potential measurements of $G_{0}-$ Ta foil system 
(2) Leakage of electrons from dynode-to $-G_{0}$ space into collector. This effect is found to be also approximately $1 \%$ at worst (with thick foil dynode), when $\mathrm{V}_{\mathrm{C}}=+90$ volts. Thus, measured $\delta_{\mathrm{t}}$ may be too large by . 01 due to this effect. At $\mathrm{V}_{\mathrm{C}}=0$, the effect is not noticeable.

The loss of backscattered primaries through the top aperture of the $G_{0}$ cup can be estimated by considering that the backscattering coefficient of the composite films of $\mathrm{Al}_{2} \mathrm{O}_{3}+\mathrm{Al}+$ alkali halide should be near .15 (that of $\mathrm{Al}_{2} \mathrm{O}_{3}$ ). ${ }^{31}$ The solid angle subtended by the aperture is approximately .019 steradian. The angular dis tribution of backscattered electrons does not appear to have been measured at the energies of interest. If one assumes an isotropic distribution, the fraction of electrons lost through the aperture is approximately .0005 . Even if the distribution were not isotropic, a wide margin still exists before this effect becomes significant in the measurement of $I_{p}$, and it will therefore be neglected.

The behavior of the $\left(C+G_{1}\right)$ assembly has been studied by measuring $\delta_{t}$ and $\eta_{t}$ of a standard $\mathrm{Al}_{2} \mathrm{O}_{3}+\mathrm{Al}$ substrate. Measurements have been carried out with the aperture of the collector Faraday cup covered by $\mathrm{Al}$ foil, and subsequently, without opening the vacuum system, with the aperture open. The results obtained are shown in Table 10. The definitions of Eqs. (17) through (19) are used without further corrections.

Table 10

\begin{tabular}{|c|c|c|c|c|c|c|c|}
\hline \multirow{2}{*}{ Dynode } & \multicolumn{3}{|c|}{} & \multicolumn{3}{|c|}{ Aperture closed } & \multicolumn{3}{c|}{ Aperture opened } \\
\cline { 2 - 8 } & $\mathrm{E}_{\mathrm{p}}$ & $\delta_{\mathrm{t}}$ & $\eta_{\mathrm{t}}$ & $\delta$ & $\delta_{\mathrm{t}}$ & $\eta_{\mathrm{t}}$ & $\delta$ \\
\hline \multirow{3}{*}{ Substrate } & 4 & .64 & .176 & .46 & .575 & .204 & .37 \\
\cline { 2 - 8 } & 8 & 1.7 & .79 & .91 & 1.43 & .865 & .57 \\
\cline { 2 - 8 } & 12 & 1.48 & .87 & .61 & 1.3 & .96 & .34 \\
\hline
\end{tabular}


A previous measurement carried out with an empty dynode holder shows that approximately . $02 \mathrm{I}_{\mathrm{p}}$ gets scattered from the collector cup into the dynode area through the aperture. From Table 10, considering the case for $E_{p}=12 \mathrm{keV}$ to be the one closest to the case of an empty holder, we see that the change in $\eta_{t}$ when opening the aperture is given by

$$
\Delta \eta_{\mathrm{t}}=\mathrm{a}-.02
$$

where "a" is the fraction of electrons scattered back by the aperture cover into the dynode. The value of "a" is then .11 , causing a change in $\delta$ of .27 . The reflection yield of the $\mathrm{Al}$ substrate is then $.27 / .11=2.4$ which is quite reasonable for a high average angle of incidence. ${ }^{3}$

When the aperture is opened, the $.02 \mathrm{I}_{\mathrm{p}}$ backscattered will result in an approximate error of $.048 \mathrm{I}_{\mathrm{p}}$, i. e. , the measured yield is too high by approximately . 05. At lower $E_{p}$, the error is expected to be lower for the following reasons:

(1) The transmission of the film is lower so that the absolute error should be roughly proportional to $\eta_{t}$.

(2) The electrons entering through the aperture have a broader angular distribution, most likely reducing the fraction of electrons coming back out.

The measurements at $\mathrm{E}_{\mathrm{p}}=4$ and $8 \mathrm{keV}$ are in quite good agreement with the above interpretation of the results for $12 \mathrm{keV}$.

When a similar measurement is carried out on a substrate $+500 \AA \mathrm{KCl}$, it is found that the reflection yield for backscattered electrons is approximately 9 , leading to measured yields which are too high by approximately .2 (in absolute value). For materials with higher yields than $\mathrm{KCl}$, this factor would become .3 perhaps.

It appears safe then to state that measured yields will be too high by approximately $.3 \eta_{t}$ in absolute value due to backscattering from the collector. This is a conservative estimate based on the worst possible case (CSI). 
The effect of the grid $G_{1}$ can be estimated as follows: In the measurement of $\eta_{\mathrm{t}}$, the current intercepted will be roughly .03 $\eta_{\mathrm{t}} \mathrm{I}$. If one takes a maximum $\delta$ of 1.5 for wide angle of incidence ${ }^{3}$ on $\mathrm{Ni}$, the value of $\eta_{t}$ will be too low by $.045 \eta_{\mathrm{t}}$. In measuring $\delta_{\mathrm{t}}$ with $\eta \simeq .25$, the effect of backscattering on the secondary generation of the dynode will be $.03 \eta_{\mathrm{t}} \mathrm{I}_{\mathrm{p}} \eta \times 9$, or in absolute value $\eta_{\mathrm{t}}$ will be too large by approximately $.07 \eta_{\mathrm{t}}$. In finding $\delta$, the measured value is too high by approximately $.1 \eta_{\mathrm{t}}$.

A summary of the errors studied can be put in the following approximate form: True $\delta$ may range between (measured $\delta$ ) and (measured $\left.\delta-.4 \eta_{\mathrm{t}}-.01\right) / 1.01$, or approximately (.99 measured $\left.\delta-.4 \eta_{\mathrm{t}}-.01\right)$. This is the worst case corresponding to a high yield material and experimental results should be better than the above estimate for low yield materials.

b. Calibration of Electronic System and Evaluation of Random Errors The Keithly 601 electrometers used for dc measurements have an accuracy rating of $1 \%$ of full scale. They are found to be within this specification. Readability of the scales is within $\pm .5 \%$ of a full scale reading. With readings made in the average at half scale, one can roughly estimate errors in finding $\delta_{t}$ and $\eta_{t}$ as being $\pm 2 \%$ each. Since in the composite films $\delta_{t}$ is always a few times larger than $\eta_{t}$, we take the error in the former as being dominant, making some allowance for the latter. One can then say that random errors in the meas urement of dc yields are approximately $\pm 3 \%$.

For pulsed measurements, the aim has been set for operation with primary pulses of the order of $10^{-14}$ coulombs at reasonably good signal-to-noise ratio. With this amount of primary charge in an area of $5 \mathrm{~mm}^{2}$, if yield is $\delta$ and all electrons leaving result in trapped holes (which is clearly not the case as seen from experiments indicated above) the voltage developed across $500 \AA$ of 
a material with a relative dielectric constant of 2 is given by

$$
\mathrm{V}=\frac{\mathrm{Q}}{\mathrm{C}}=\frac{\mathrm{Q} \cdot \mathrm{d}}{\epsilon \epsilon_{0} \mathrm{~A}}=\frac{10^{-14} \times \times 5 \times 10^{-8}}{2 \times 8.85 \times 10^{-11} \times 5 \times 10^{-6}} \simeq .5 \times 10^{-5} \delta \text { volts (M. K. S.) }
$$

For $\delta$ with a maximum of 10 , it is clear that a very large number of pulses can be used before any appreciable voltage is built up.

The initial approximate calibration has been carried out by applying a simultaneous step of voltage to the inputs of $A$ and $B_{1}$ through separate $2 p f d \pm 2 \%$ capacitors. The total charge delivered is $Q=V C$, provided that the input capacitance of the amplifiers is much larger than $C$. This capacitance $C_{\text {in total }}$ is given by the sum of wiring, electrode, and stray capacitance plus the feedback capacitor times open loop gain of the amplifier. This makes $C_{\text {in total }}$ equal to a few thousand pfd. In such case, a step of $5 \mathrm{mV}$ into 2 pfd corresponds to $10^{-14}$ coulombs of charge. The gains of the amplifiers were set so that one pulse produced $2 \mathrm{~cm}$ deflection in the $I_{p}$ channel of the oscilloscope $(10 \mathrm{mV} / \mathrm{cm}$ sensitivity setting) and $1 \mathrm{~cm}$ deflection in the $\mathrm{I}_{\mathrm{C}+\mathrm{G}_{1}}$ channel (set at $100 \mathrm{mV} / \mathrm{cm}$ ). In this way, $1 \mathrm{~cm}$ deflection corresponds to $10^{-14}$ coulombs in the corresponding channel.

This simultaneous calibration of both channels is approximate and not sufficiently good for the present case in which $I_{p}$ is obtained by the sum of two currents which may be large and of opposite signs. The reason for the inaccuracy lies in the fact that the charge delivered by the 2 pfd capacitor is collected at the feedback capacitor $\mathrm{C}_{\mathrm{fb}}$ in proportion to $\mathrm{C}_{\mathrm{fb}} \times$ open loop gain/ $\mathrm{C}_{\text {in total }}$ which is not the same for the two amplifier channels. The best calibration is then carried out by switching amplifiers $A$ and $B_{1}$ and the following stages back and forth from one channel to the other while adjusting $R_{3}$ (Fig. 20) until a unique value of $\delta_{t}$ is obtained from a high gain film (CsI, $500 \AA$ A, $7 \mathrm{keV}$ ). This calibration, carried out at high charge per pulse $\left(I_{p} \approx 10^{-13}\right.$ coulcomb in $\left.5 \mu \mathrm{sec}\right)$ for good $S / N$, allows consistent measurements of $\delta_{t}$ under switching to better than $\pm 1 \%$ over a large range of yields. 
Figure 24 shows a typical oscilloscope pattern in a measurement of $\delta_{t}$. The top trace corresponds in magnitude to $I_{p}$. One $\mathrm{cm}$ is equivalent to $2 \times 10^{-14}$ coulomb. The bottom trace corresponds to the magnitude of $\mathrm{I}_{\mathrm{C}+\mathrm{G}_{1}}$, with $1 \mathrm{~cm}$ corresponding to $5 \times 10^{-14}$ coulombs. Horizontal scale is $20 \mu \mathrm{sec} / \mathrm{cm}$. The film measured is $500 \AA \mathrm{KCl}, \mathrm{E}_{\mathrm{p}}=7 \mathrm{keV}$, state of charge not defined. The oscilloscope traces can be read to approximately $\pm .05 \mathrm{~cm}$. Assuming that in the measurements both $\mathrm{I}_{\mathrm{p}}$ and $\mathrm{I}_{\mathrm{C}+\mathrm{G}_{1}}$ give about the same deflection, but the latter represents $\delta_{\mathrm{t}}$ times more charge, the readings of $\delta_{\mathrm{t}}$ approximately good to $\pm .1 \delta_{\mathrm{t}} /$ deflection in $\mathrm{cm}$. Usually measurements will be carried out with deflections of 3 to $5 \mathrm{~cm}$, giving errors due to reading of about $\pm .025 \delta_{t}$.

In order to estimate the electronic noise of the system at the indicated level of signal, 20 measurements on the indicated film have been carried out. Their mean is 4.95 and the standard deviation is . 16. Since the expected error in reading the oscilloscope is approximately \pm .125 , it appears that the noise contribution to the error is dominant. If one assumes that in taking two or three measurements of $\delta_{t}$ for a given set of parameters the average of the measurements will be contained within \pm one standard deviation, one can specify an approximate margin of error for pulsed measurements as being . 16/4.95, or $\pm 3 \%$ at typical measurement levels. The dc value of $\delta_{t}$ for the same film is measured to be $5.07 \pm .15$. The state of charge of the film during this measurement is undetermined, but it should be the same for both, as the dc measurement was done first and discharge time constants are long. The agreement between the two methods is good.

Finally, the accelerating high voltage has to be considered. The potential is measured with a Keithley electrometer fitted with a $30 \mathrm{~K}$ volt probe. The assembly has been calibrated with. . . $5 \%$ Singer electrostatic voltmeter. Resetability is to within \pm 30 or 40 volts at the more critical energies below maximum yield. The error introduced is small and can be neglected in comparison with the other errors indicated above. 


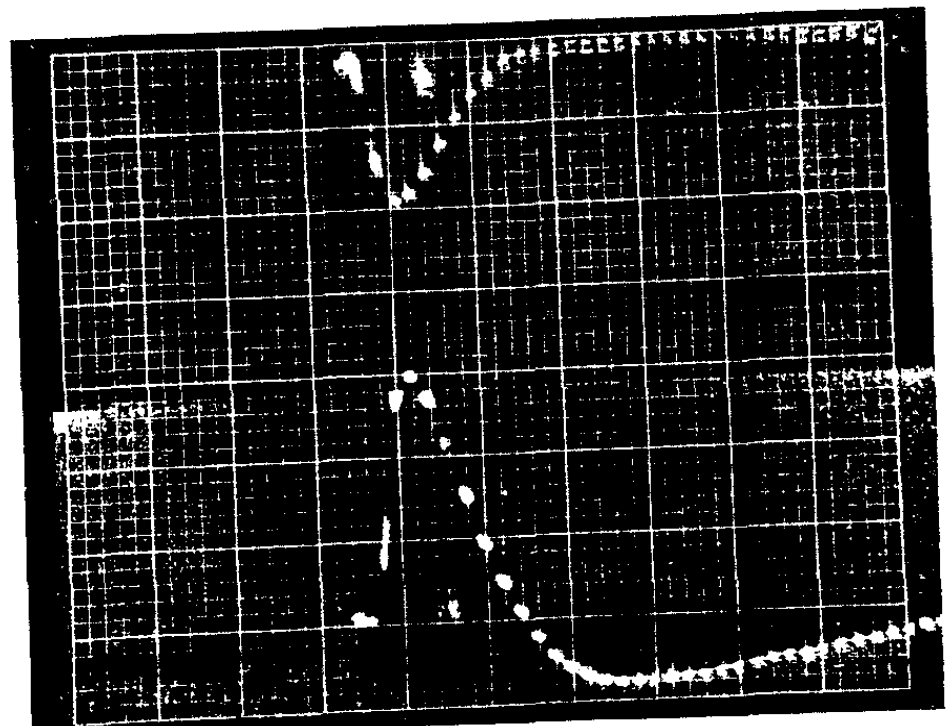

$\overline{987 A 27}$

Fig. 24--Typical oscilloscope pattern in the measurement of pulsed $\delta_{t}$.

Vertical scale: $2 \times 10^{-14}$ coulomb/cm at peak, horizontal: $20 \mu \mathrm{sec} / \mathrm{cm}$ 


\section{Preliminary Measurements}

\section{a. Yield of Substrates}

The secondary emission yield of the standard substrates used for the present measurements has been measured repeatedly with a dc beam. Pulsed beam measurements have been carried out several times with identical results, as expected. The thickness of the $\mathrm{Al}_{2} \mathrm{O}_{3}$ support film(1000A) and that of the $500 \mathrm{~A}$ Al evaporation should be correct with in a few percent for all the different batches of substrates. No special effort has been made to control the surface characteristics of the Al layer. After evaporation in an oil diffusion pumped system at pressures of about $10^{-6}$ torr, films are stored in a dry box. Figure 25a shows $\delta$ vs. $E_{p}$ for films of different batches. The spread in results is much larger than the expected errors discussed above, and it is believed to be due to differences in the surface characteristics of the Al layer. Proof of the uniformity of film thickness lies in the uniformity of $\eta_{t}$ for the same films as shown in Fig. 25 b.

\section{b. Effect of Pressure and Evaporation Rate on Yield of Alkali Halides} Several films of CsI have been evaporated at pressures between $1.5 \times 10^{-5}$ torr and $8 \times 10^{8}$ torr and at rates between $1 \stackrel{0}{\mathrm{~A}} / \mathrm{sec}$ and $10 \mathrm{~A} / \mathrm{sec}$. The dc yields have been found invariant under the above changes, within the expected errors of the measuring system. Similar measurements for $\mathrm{KCl}$ and $\mathrm{NaF}$ seem to indicate a small effect due to pressure during evaporation when this was carried out at pressures of $10^{-7}$ torr or higher, but no clear effect due to evaporation rate when the latter is in the neighborhood of 1 to $10 \mathrm{~A} / \mathrm{sec}$. It was decided to carry out all the evaporations at near the best vacuum obtainable in the system, about $2 \times 10^{-8}$ torr and to use an evaporation rate within the range indicated above. The vacuum system was always be opened to dry nitrogen, with relative humidity in the enclosing plastic box of less than $1.5 \%$. 


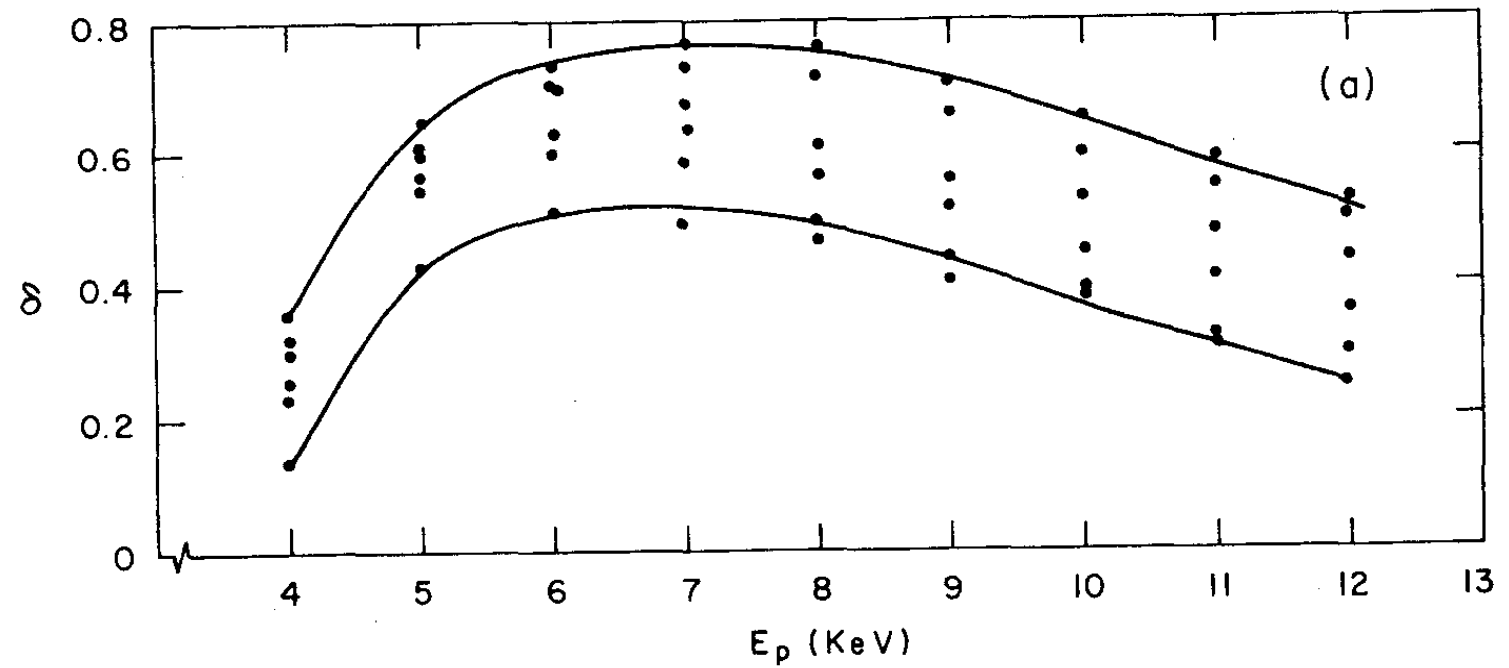

$\stackrel{1}{\circ}$

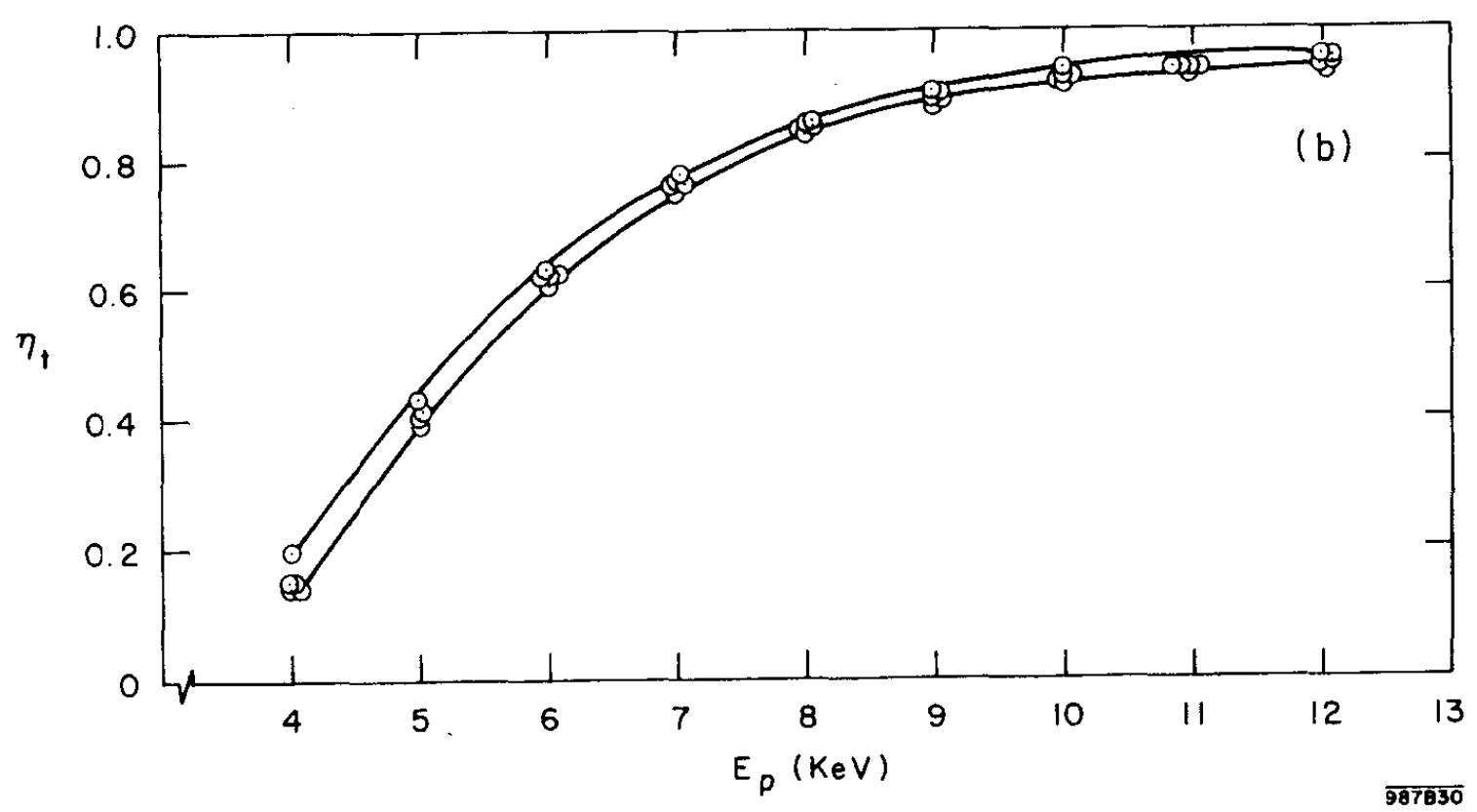

FIG. 25--Secondary yield $\delta$, (a), and transmission coefficients $\eta_{\mathrm{t}}$, (b), for $1000 \AA \mathrm{Al}_{2} \mathrm{O}_{3}+500 \AA \mathrm{Al}$ substrates 


\section{Measurements on Alkali Halides}

Measurements of secondary yield on the four selected alkali halides have been carried out. For a given material, six films with thickness of approximately $125,250,375,500,750$, and $1000 \AA$ have been investigated. It has been found that substrates can be reused by rinsing off the alkali halide deposit by immersion in water. The low secondary emission characteristics of the substrate are recovered. The procedure can be repeated three of four times before damage to the $\mathrm{Al}_{2} \mathrm{O}_{3}$ layer becomes evident (lifting off from support ring). Substrates have only been reused within measurements of a given alkali halide, though.

Preliminary measurements carried out by evaporation on top of an already existing layer of salt on which measurements have previously been made give inconsistent results, depending on past history. For this reason new evaporations are required every time.

After checking the instruments by measuring $\delta$ for the substrate at a selected value of $\mathrm{E}_{\mathrm{p}}$, pulsed and dc, the desired thickness of halide salt is evaporated. Immediately pulsed measurements at energies between 4 and $12 \mathrm{keV}$ are taken. At each point, 3 pulses of 2 to $5 \times 10^{-14}$ coulombs have been used. The average of the results of using Eq. (17) will be $\delta_{t}$, the measured transmission yield for an uncharged film. The fact that a freshly evaporated film is uncharged has been checked by photoemission measurements of CsI by T. DiStephano ${ }^{48}$ at Stanford University.

Next, dc measurements at some energies of interest are carried out at primary currents of 5 to $10 \mathrm{nA}$. These are to be compared to $\delta_{\mathrm{t}}$ in order to see if there is any enhancement of the yield at dc. Subsequently, $\eta_{\mathrm{t}}$ is measured at dc.

The only exception to the above procedure for carrying out measurements is the case of CsI in which a complete set of low level $\left(\mathrm{I}_{\mathrm{p}}<1 \mathrm{nA}\right)$ dc measurements was carried out prior to developing a satisfactory pulsed method. It has been 
subsequently determined that those measurements are completely equivalent to pulsed results as no dc enhancement of yield is observable in CsI films.

The statistical error in the measurement of $\delta_{t}$ has been estimated to be $\pm .03 \delta_{t}$. The corresponding error in measuring $\eta_{t}$ is approximately $\pm .02 \eta_{t}$. In addition, the systematic errors in $\delta$ can be roughly as large as $\left(-.01 \delta-.4 \eta_{\mathrm{t}}-.01\right)$. The points corresponding to $\delta_{\mathrm{t}}-\eta_{\mathrm{t}}$ will be plotted in a graph and bars between $\left(1.03 \delta_{t}-.98 \eta_{t}\right)$ and $\left(.98 \delta_{t}-1.42 \eta_{t}-.01\right)$ will indicate the range over which the true $\delta$ is expected to exist. Since the systematic errors cannot be determined with sufficient accuracy, they are introduced as giving rise to a possible error rather than being used as correction factors.

Measurements are all at room temperature. The filament of the electron gun is turned off between resetting of voltages in order to minimize temperature changes. Reversible changes in yield have been observed after approximately 30 minutes of having the filament on, with the beam cut off.

Figures 26 through 29 show the results of measuring secondary emission yield in transmission for $\mathrm{CsI}, \mathrm{KCl}, \mathrm{NaF}$, and $\mathrm{LiF}$. Note that the vertical scale has been magnified by a factor of two in the case of LiF. Also shown are theoretical results to be discussed in Chapter III. The materials used for the evaporations can be described as follows:

CsI: $99.99 \%$ certified purity, Research Organic/Inorganic Chemical Co. $\mathrm{KCl}$ : Optical Quality, Harshaw (expected purity 99.9\%).

NaF: $99.99 \%$ certified purity, Research Organic/Inorganic Chemical Co.

LiF: Suprapur, Merck (expected purity 99.9\%).

Handling has been done with stainless steel tools, ultrasonically cleaned in acetone and ethanol. Evaporations were made from Mo or Ta boats, cleaned and fired in vacuum. Outgassing of the powder or small crystals by heating in $2 \times 10^{-8}$ torr initial vacuum has been carried out before the actual evaporations. 

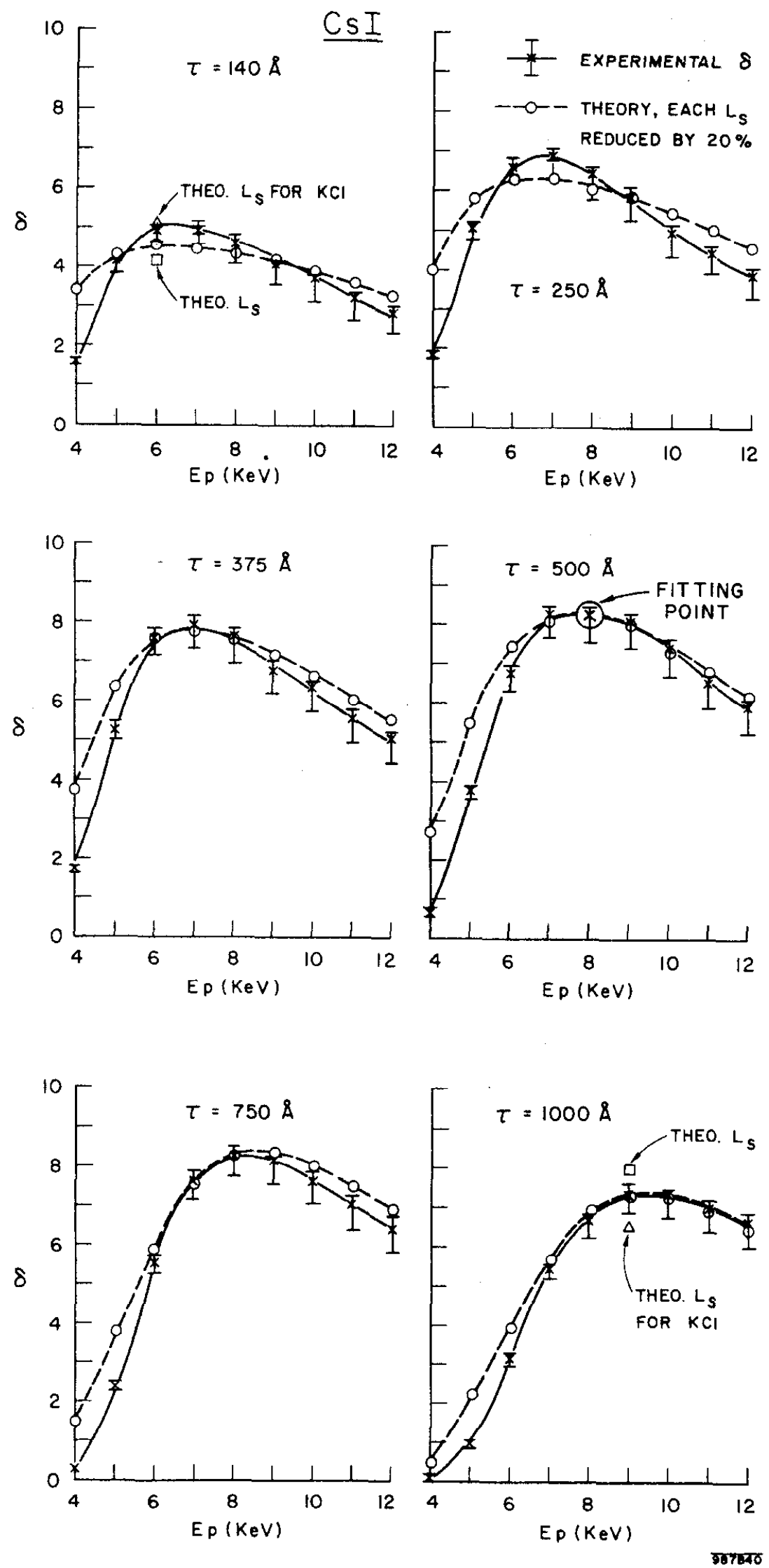

FIG. 26--Experimental and theoretical secondary yield vs. primary energy for 6 different thicknesses of CsI films. Calculated yields fitted to experimental results only at $\tau=500 \AA$, $\mathrm{E}_{\mathrm{p}}=8 \mathrm{keV}$. 

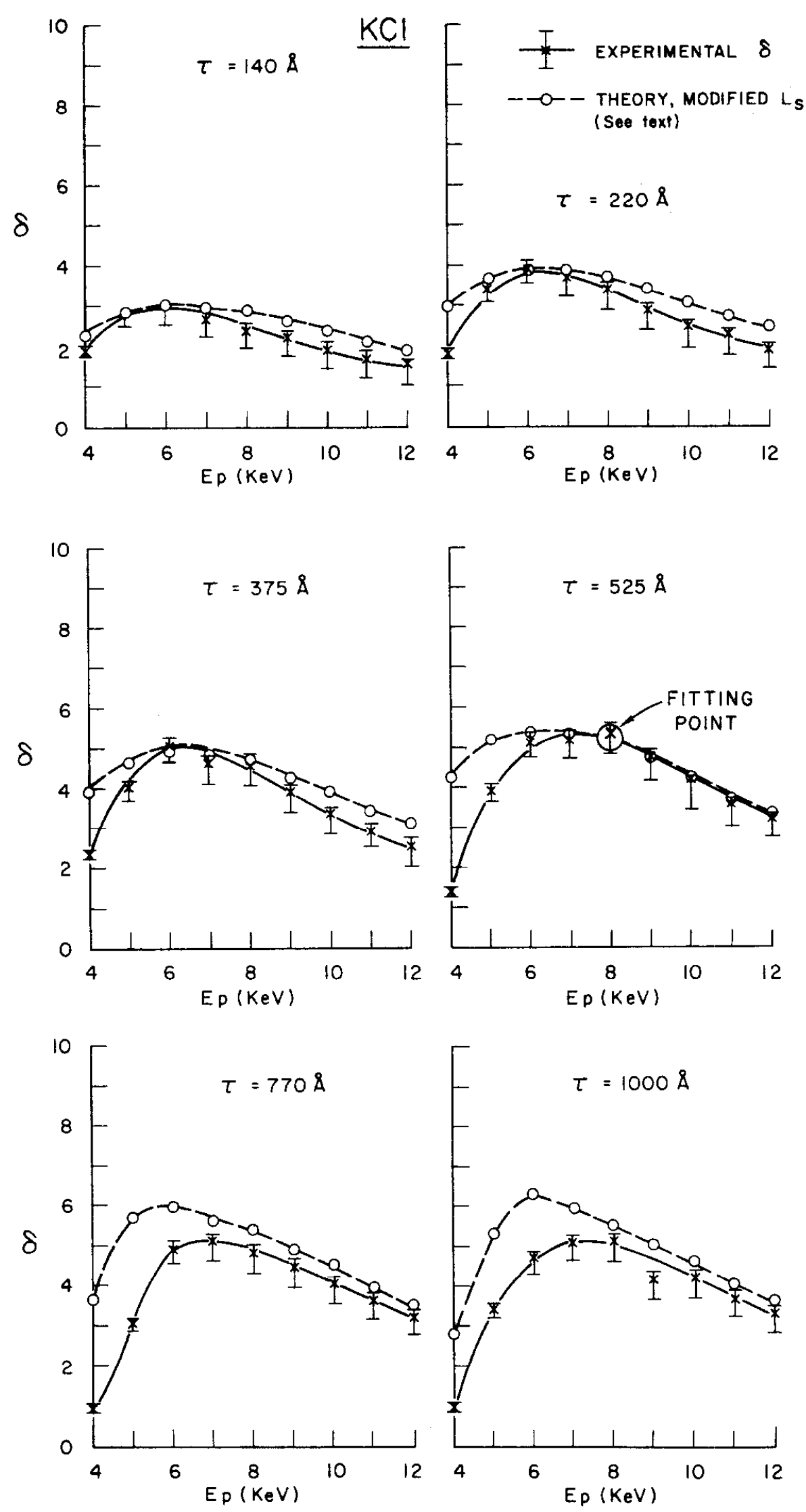

$\overline{58784}$

FIG. 27--Experimental and theoretical secondary yield vs. primary energy for 6 different thicknesses of $\mathrm{KCl}$ films. Calculated yields fitted to experimental results only at $\tau=525 \AA$, $\mathrm{E}_{\mathrm{p}}=8 \mathrm{keV}$. 

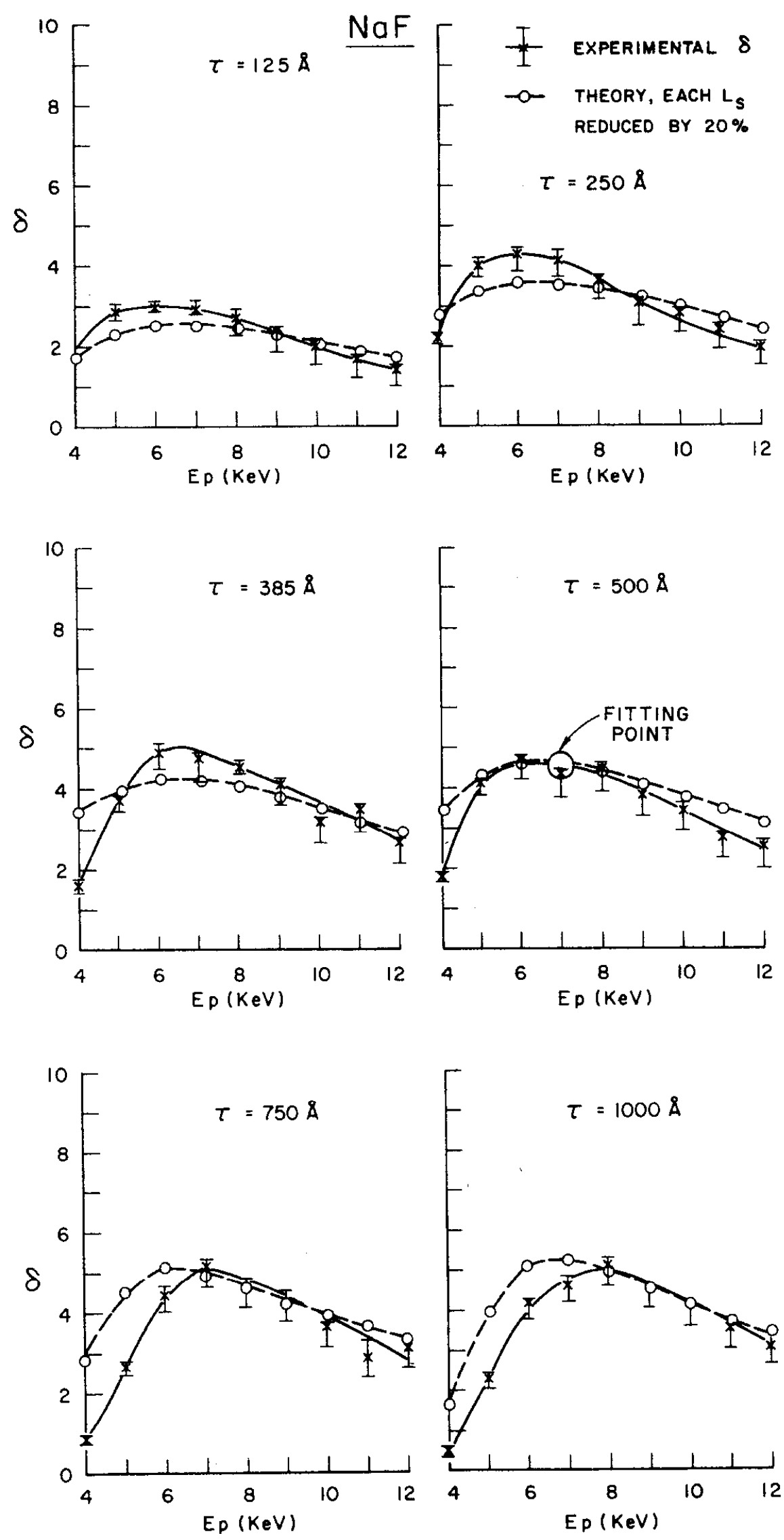

Griat

FIG. 28--Experimental and theoretical secondary yield vs. primary energy for 6 different thicknesses of NaF films. Calculated yields fitted to experimental results only at $\tau=500 \AA$, $E_{p}=7 \mathrm{keV}$. 

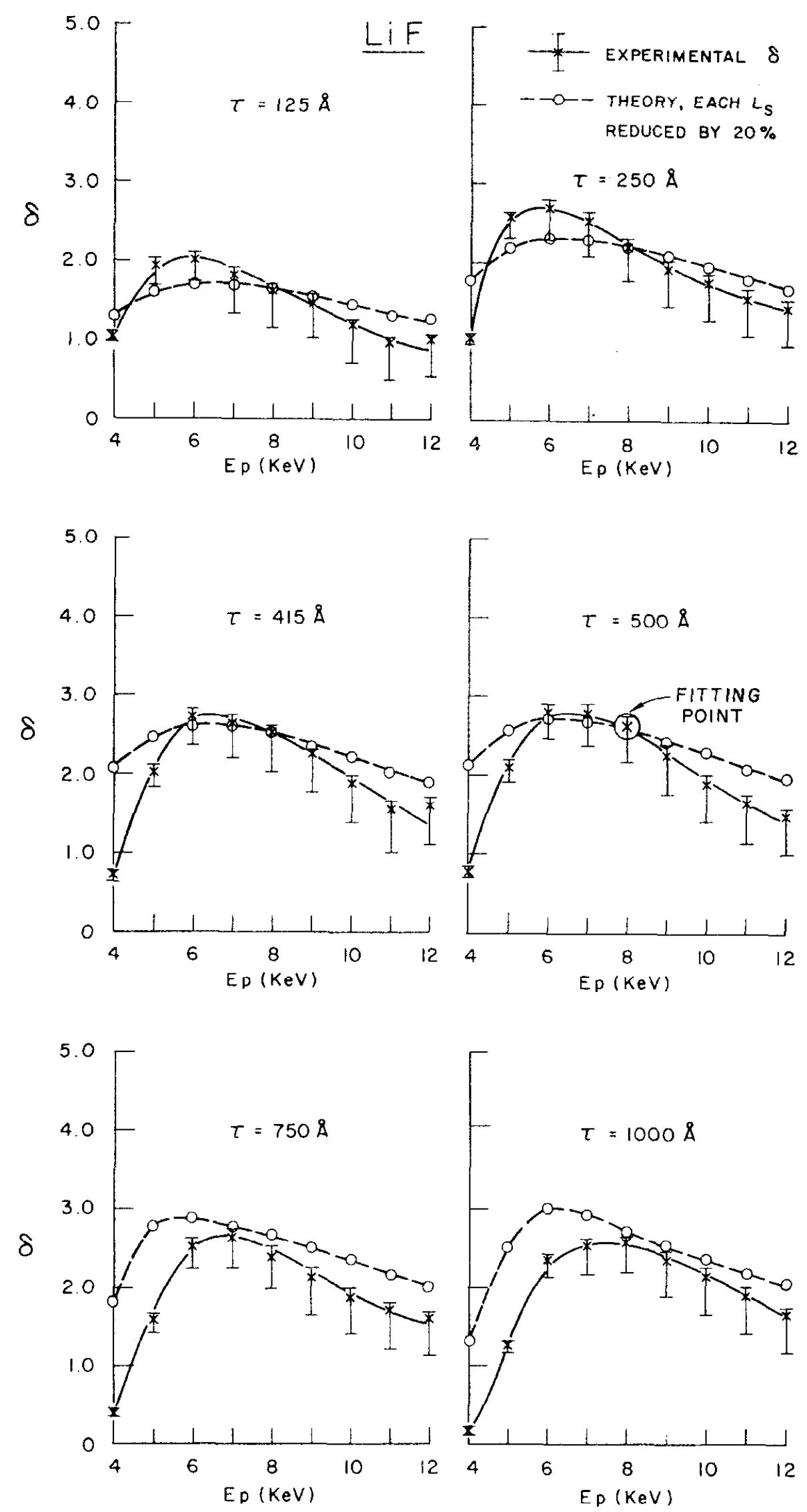

$\overline{987 \overline{843}}$

FIG. 29--Experimental and theoretical secondary yield vs. primary energy for 6 different thicknesses of LiF films. Calculated yields fitted to experimental results only at $\tau=500 \AA$, $\mathrm{E}_{\mathrm{p}}=8 \mathrm{keV}$. 
The four materials are presented in order of decreasing maximum yield, which ranges from approximately $\delta \simeq 8.3$ for $750 \AA$ of $\mathrm{CsI}$ at $\mathrm{E}_{\mathrm{p}}=8 \mathrm{keV}$ to $\delta \simeq 2.75$ for $400 \AA$ of $\mathrm{LiF}$ at $\mathrm{E}_{\mathrm{p}}=6 \mathrm{keV}$.

The shape of the $\delta$ vs. $E_{p}$ curves shown is not unlike those for reflection secondary emission. They are characterized by low $\delta$ at low $E_{p}$, and as $E_{p}$ increases, a maximum in yield is reached followed by a decrease.

One can see qualitatively how this comes about in the present case by examining Fig. 4, for example, in conjunction with Fig. 26, both for CsI. For any chosen thickness of material, and assuming an exponential escape probability, the existence of the maximum in yield is easy to see by looking at the curves of $\mathrm{dE} / \mathrm{dx}$ for different primary energies. The shift of the position of maximum yield with thickness is also quite clear: For a film of thickness $\tau=250 \AA$ A $\left(10 \mu \mathrm{g} / \mathrm{cm}^{2}\right)$, a maximum yield should be expected near $\mathrm{E}_{\mathrm{p}}=6 \mathrm{keV}$, while at $\tau=1000 \AA$, maximum yield might be more likely at about $\mathrm{E}_{\mathrm{p}}=10 \mathrm{keV}$, if an exponential escape depth of a few hundred $\stackrel{\circ}{\AA}$ applies.

It is also to be expected from Fig. 4 that at $\mathrm{E}_{\mathrm{p}}=4 \mathrm{keV}$ the secondary yield will at first increase as thickness increases, while later it will decrease to a low value as $\tau$ goes towards $1000 \AA$. At $\mathrm{E}_{\mathrm{p}}=12 \mathrm{keV}$, the linear loss correction of Fig. 4 indicates a uniform energy loss up to $\tau \simeq 900 \AA$, and with the assumption of an exponential escape depth the yield at that energy should be increasing with thickness, with the possible exception of the $\tau=1000 \AA$ case. These considerations are well verified by the experimental results of Fig. 26 and hold quite well for the other materials tested, although the case of CsI is the clearest one due to its high density and atomic number allowing very distinct changes in $\mathrm{dE} / \mathrm{dx}$ with changes in thickness.

It must also be remarked that the value of $E_{p}$ required for maximum yield is much higher than in reflection, mainly because of the presence of the necessary substrate. 
The results of Figs. 26 through 29 correspond to the secondary yield of uncharged films. As indicated above, the measurements on CsI were originally carried out at low level dc, but careful comparison between pulsed results for two separate new $500 \AA$ films of CsI and subsequent dc measurements, and pulsed on top of dc, failed to show any enhancement in yield due to charging even at primary currents of more than $10 \mathrm{nA}$ on an area of approximately $5 \mathrm{~mm}^{2}$.

For $\mathrm{KCl}$ and LiF films of up to $500 \AA$ thickness, no yield enhancement has been observable within the resolution capabilities of the measuring setup.

In the case of $\mathrm{NaF}$, however, yield enhancement was very apparent. For $\tau=385 \mathrm{~A}, \mathrm{E}_{\mathrm{p}}=8 \mathrm{keV}$, pulsed yield for a new film was approximately 5 , while it went to $\delta \simeq 6$ at $d \_$and when a pulsed measurement was carried out simultaneously. Ten minutes after beam turn off, pulsed $\delta$ went to 5.6 and after ten more minutes to 5.3 . The process was found to be quite reproducible. In a $1000 \AA$ film of the same material, also at $8 \mathrm{keV}$, the yield changed from 4.9 to 6 in the same manner.

One can speculate on the difference between the NaF films and those of the other three materials by realizing that field enhancement may be due to the inability of low energy electrons (bottom of conduction band) to reach trapped holes. Instead, if the film contains many imperfections due to some dissociation at the time of evaporation, f-centers could be formed. Some evidence of dissociation for the present NaF films was apparent as will be discussed below in the section related to contact potential measurements. Figure 30 shows the transmission coefficient $\eta_{\mathrm{t}}$ for a typical substrate and for the same CsI films of Fig. 26. Expected errors are $+2,-5 \%$. A line indicating the approximate $\eta_{t}$ and $E_{p}$ for maximum secondary yield at each different thickness shows that the maximum occurs at $\eta_{\mathrm{t}} \simeq .5$ for all thicknesses. From Kanter's results, ${ }^{6}$ one sees that the average energy of the transmitted electrons is approximately $.65 \mathrm{E}_{\mathrm{p}}$ so that 


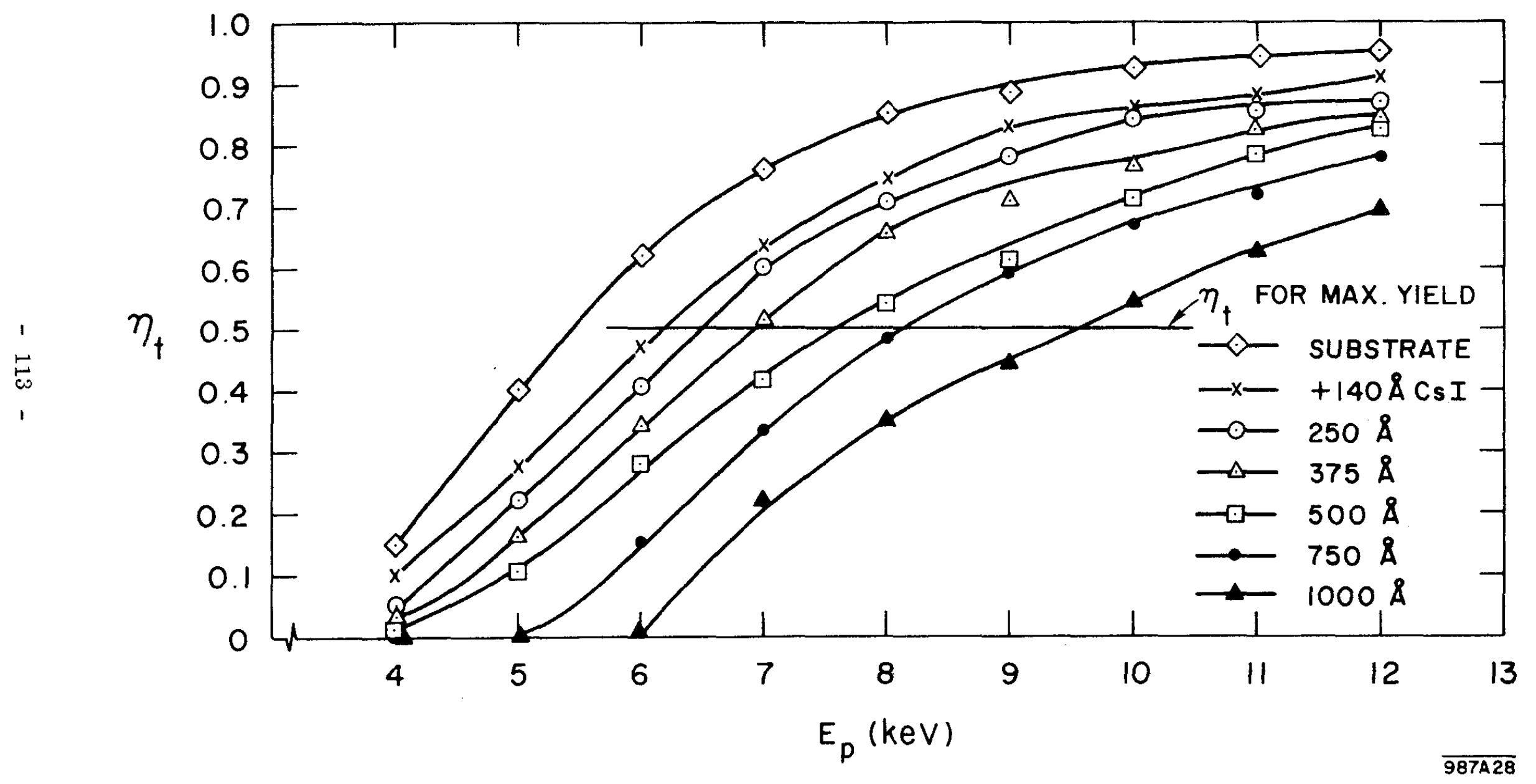

FIG. 30--Transmission coefficient for standard substrate + CsI films 
at the point of maximum yield, the primary beam has lost approximately $75 \%$ of its energy in the compound film. The only point of this observation is to note that maximum yield does not occur at an energy such that primary beam absorption is near $100 \%$.

\section{Measurement of Energy Distribution in Uncharged Films}

The measurement of the energy distribution of secondary electrons from insulators has always been somewhat problematic due to charge accumulation at or near the exit surface. By using low primary currents, Geyer ${ }^{71}$ was able to make some measurements of energy distribution for different thicknesses of $\mathrm{NaCl}$, in reflection. Petzel ${ }^{72}$ published curves of energy distribution for single crystals of $\mathrm{KCl}$ with a correction for contact potential. Comparison of the two results shows a definite discrepancy in the peak of the energy distribution from less than .05 eV (Geyer) to $\approx 1.5 \mathrm{eV}$ (Petzel) which makes the results somewhat doubtful, as the two materials are not very different. These difficulties point out the need for a system capable of measuring contact potential differences (cpd) simultaneously to making retarding potential measurements. In addition, for the purposes of the present investigation, energy distribution measurements should be carried out on films known not to be charged.

Three alternative approaches have been considered:

(1). Spherical collector with a second low energy electron gun for probing at the exit surface of the alkali halide film, in a manner similar to the one used by Boll. ${ }^{73}$ This method was considered not useful for the present investigation because it does not allow simultaneous measurements of cpd and energy distribution. Also, the measurement with a second gun may disturb the surface which one is trying to study. One advantage does exist, however, in the ability to use a spherical collector geometry, making energy distribution measurements independent from the angular distribution of the emitted electrons. 
(2) Planar geometry in the collector-emitter structure, with a transverse electron beam passing between the collector and emitter planes. Deflection of the transverse beam would indicate the field between the two planes.

(3) Planar geometry with a Kelvin probe at the collector plane.

An examination of the second and third alternatives indicated certain advantages of the Kelvin probe method: possibility of very good sensitivity without critical alignment problems; ease of calibration at different collector-to-dynode spacings; measurements of cpd can be made of a small well-defined area of the film; and recording of results is simpler. This approach to cpd measurements was therefore chosen.

\section{Principles of Operation}

\section{a. Kelvin Probe}

Figure 31 shows schematically a physical setup for the measurement of potential difference (pd) by a Kelvin probe. A metallic substrate holding a thickness $\mathrm{x}_{1}$ of material with a free charge distribution $\rho(\mathrm{x})$ and relative dielectric constant $\epsilon$ is connected to ground through a battery $\mathrm{V}_{\mathrm{D}}$ which includes the contact potential difference (cpd) between the metallic substrate A and the vibrating capacitor plate B. The latter is connected to ground via $R_{L}$ in parallel with $C_{L}$.

In the absence of free charge $\rho(\mathrm{x})$, the problem can be treated as the case of a simplecapacitor which for a certain spacing $d$ will have an induced charge at the plate $\mathrm{B}$ equal to $-\epsilon_{0} \epsilon \mathrm{V}_{\mathrm{D}} /\left[\mathrm{x}_{1}+\left(\mathrm{d}-\mathrm{x}_{1}\right)\right]$. For $\rho(\mathrm{x}) \neq 0$, it will now be shown that the same simple treatment can be used for any arbitrary $\rho(x)$ and any film thickness $x_{1} \ll d$.

For a sheet of charge $q$ located at $x, 0 \leq x \leq x_{1}$, the charge $Q_{B}$ induced at the plate $B$ is

$$
Q_{B}=\frac{-q x}{\left(d-x_{1}\right) \epsilon+x_{1}}
$$



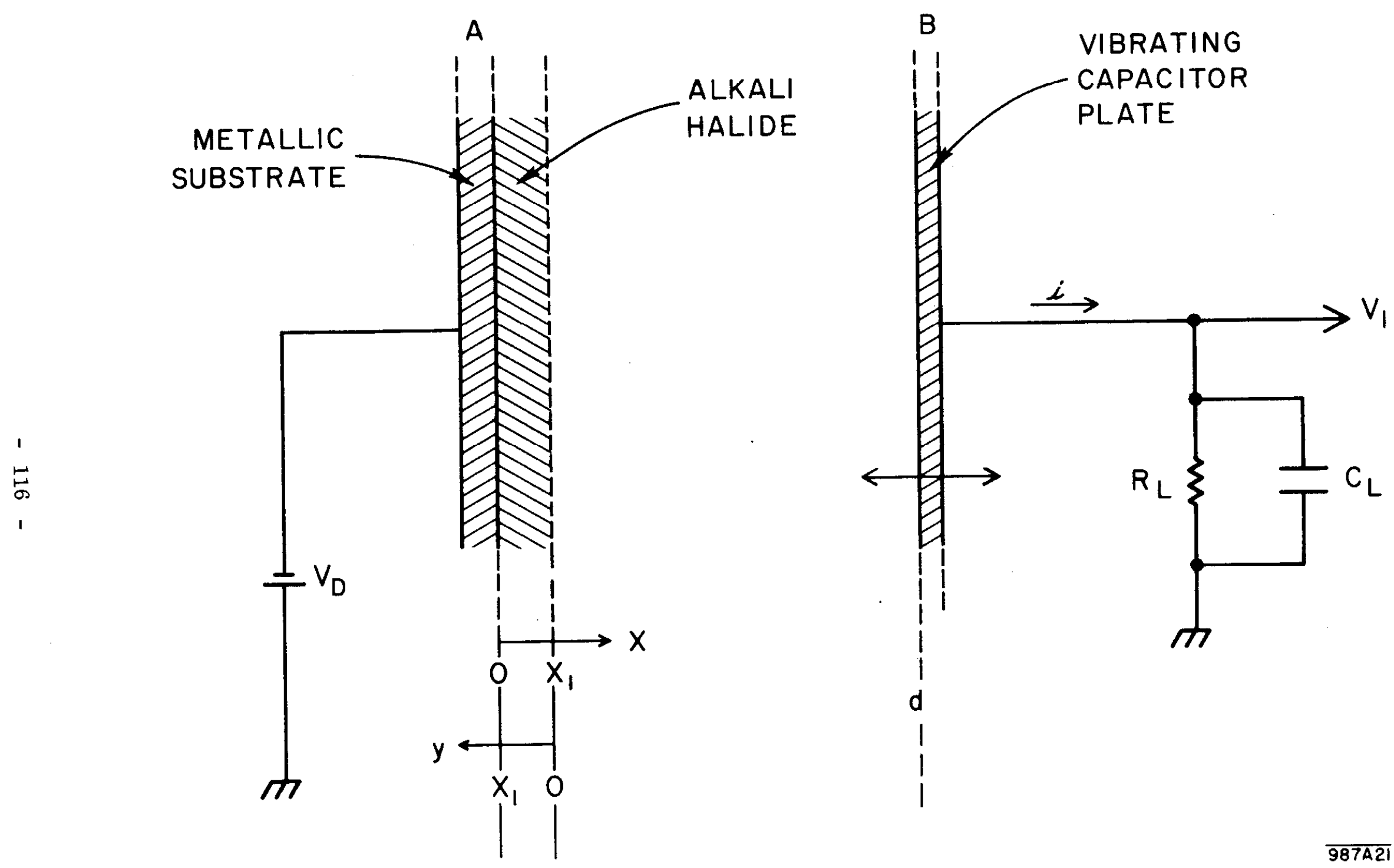
For a distribution of charge $P(x)$ the total induced charge will be

$$
\mathrm{Q}_{\mathrm{B}}=-\int_{0}^{\mathrm{x}_{1}} \frac{\mathrm{x} \rho(\mathrm{x}) \mathrm{dx}}{\left(\mathrm{d}-\mathrm{x}_{1}\right) \epsilon+\mathrm{x}_{1}} \text {. }
$$

We wish now to see if the charge induced at $B$ due to an increment of voltage $V_{D}^{\prime}$ at $A$ equal to the difference of potential between the two faces of the insulator film is the same as $Q_{B}$ of Eq. 20 .

The constant electric field $E$ for $x_{1} \leq x \leq d$ can be obtained from Eq. (20):

$$
E=\frac{-Q_{B}}{\epsilon_{0}}=\frac{1}{\epsilon_{0}\left[\left(d-x_{1}\right):+x_{1}\right]} \int_{0}^{x_{1}} x \rho(x) d x
$$

and the potential at $x_{1}$ is therefore

$$
V\left(x_{1}\right)=\frac{\left(d-x_{1}\right)}{\epsilon_{0}\left[\left(d-x_{1}\right) \epsilon+x_{1}\right]} \int_{0}^{x_{1}} x \rho(x) d x=V_{D}^{t} .
$$

If $V_{D}^{\prime}$ is now applied at $A$, it will result in an induced charge $Q_{B}{ }^{\prime}$ at $B$ given by

$$
\mathrm{Q}_{\mathrm{B}^{\prime}}=-\frac{\epsilon_{0} \epsilon \mathrm{V}_{\mathrm{D}}}{\left(\mathrm{d}-\mathrm{x}_{1}\right) \epsilon+\mathrm{x}_{1}}=\mathrm{Q}_{\mathrm{B}} \frac{\left(\mathrm{d}-\mathrm{x}_{1}\right) \epsilon}{\left(\mathrm{d}-\mathrm{x}_{1}\right) \epsilon+\mathrm{x}_{1}}
$$

Thus, as long as $x_{1} \ll d$ which is the practical case, $Q_{B}$ can be used as a measure of true potential at $x_{1}$. This takes into consideration the fact that the field in the region $x_{1} \leq x \leq d$ is not really zero and takes part in the determination of $V\left(x_{1}\right)$.

With plate $\mathrm{B}$ vibrating in such a way that capacitance $\mathrm{C}$ is approximately equal to $\mathrm{C}_{0}+\mathrm{C}_{1} \cos \omega \mathrm{t}, \mathrm{C}_{1} \ll \mathrm{C}_{0}$, the instantaneous charge $\mathrm{Q}_{\mathrm{B}}$ is $\mathrm{C}\left(\mathrm{V}_{\mathrm{S}}-\mathrm{V}_{1}\right)$, where $V_{S}=V_{D}+V_{D}^{\prime}$ is the potential at the surface $x=x_{1}$. 
The charge $Q_{B}$ will obey the equations

$$
i=\frac{d Q_{B}}{d t}=C \frac{d\left(V_{S}-V_{1}\right)}{d t}+\left(V_{S}-V_{1}\right) \frac{d C}{d t}
$$

and

$$
i=\frac{d Q_{B}}{d t}=C_{L} \frac{d V_{1}}{d t}+\frac{V_{1}}{R_{L}} .
$$

The differential equation for $V_{1}(t)$ is therefore

$$
C \frac{d V_{S}}{d t}-C \frac{d V_{1}}{d t}+V_{S} \frac{d C}{d t}-V_{1} \frac{d C}{d t}=C_{L} \frac{d V_{1}}{d t}+\frac{V_{1}}{R_{L}} .
$$

For $V_{S}(t)=V_{S} U(t)$, a unit step of amplitude $V_{S}$, and $\frac{d C}{d t}=-\omega C_{1} \sin \omega t$, we proceed to change Eq. (21) into a time independent linear equation by taking $C=C_{0}$ in the first two terms(i.e., $C_{1} \ll C_{0}$ ), and $V_{S} \gg V_{1}$ so that the fourth term can be left out. These simplifications are justified by an examination of the approximate solution given below and from the actual performance of the system. We have then,

$$
\left(C_{0}+C_{L}\right) \frac{d}{d t}\left(V_{1} U(t)\right)+\frac{1}{R_{L}}\left(V_{1} U(t)\right)=-V_{S} \omega C_{1} \sin \omega t U(t)+C_{0} \frac{d}{d t}\left(V_{S} U(t)\right) \cdot
$$

Taking unilateral Laplace transforms one obtains

$$
\left(\mathrm{C}_{0}+\mathrm{C}_{\mathrm{L}}\right) \mathrm{s} \hat{\mathrm{v}}_{1}+\frac{1}{\mathrm{R}_{L}} \hat{\mathrm{v}}_{1}=-\left(\mathrm{V}_{\mathrm{S}} \omega \mathrm{C}_{1} \frac{1}{\mathrm{~s}^{2}+\omega^{2}}\right)+\mathrm{C}_{0} \mathrm{v}_{\mathrm{S}}
$$

where $\hat{V}_{1}$ is the transform of $V_{1} U(t)$.

Solving for $\hat{\mathrm{V}}_{1}$, one obtains

$$
\hat{v}_{1}=\frac{-\mathrm{V}_{\mathrm{S}} \omega^{2} \mathrm{C}_{1}}{\mathrm{C}_{0}+\mathrm{C}_{\mathrm{L}}} \frac{1}{\left(\mathrm{~s}+\frac{1}{\tau}\right)\left(\mathrm{s}^{2}+\omega^{2}\right)}+\frac{\mathrm{C}_{0} \mathrm{~V}_{\mathrm{S}}}{\mathrm{C}_{0}+\mathrm{C}_{\mathrm{L}}}-\frac{1}{\mathrm{~s}+\frac{1}{\tau}}
$$

where $\tau=R_{L}\left(C_{0}+C_{L}\right)$. 
The inverse transform is

$$
\mathrm{V}_{1}(\mathrm{t})=\frac{-\mathrm{V}_{\mathrm{S}} \omega^{2} \mathrm{C}_{1}}{\mathrm{C}_{0}+\mathrm{C}_{\mathrm{L}}}\left[\frac{\mathrm{e}^{-\mathrm{t} / \tau}}{\left(\frac{1}{\tau}\right)^{2}+\omega^{2}}+\frac{\sin (\omega t-\psi)}{\omega\left[\left(\frac{1}{\tau}\right)^{2}+\omega^{2}\right]^{1 / 2}}\right]+\frac{\mathrm{C}_{0} \mathrm{~V}_{S}}{\mathrm{C}_{0}+\mathrm{C}_{\mathrm{L}}} \mathrm{e}^{-\mathrm{t} / \tau}
$$

where the phase angle $\psi$ is equal to $\tan ^{-1}(\omega \tau)$.

Looking at the steady state signal, we find that

$$
\mathrm{V}_{1}(\mathrm{t})=\frac{-\mathrm{V}_{\mathrm{S}} \omega \mathrm{C}_{1}}{\mathrm{C}_{0}+\mathrm{C}_{\mathrm{L}}} \frac{\sin (\omega \mathrm{t}-\psi)}{\left[\left(\frac{1}{\tau}\right)^{2}+\omega^{2}\right]^{1 / 2}}
$$

The result shows that the ac signal voltage is proportional to $C_{1} /\left(C_{0}+C_{L}\right)$, so that for a given geometry and available drive, we should make $C_{L}$ as small as possible. The response increases with frequency until the neighborhood of $\omega \simeq 1 / \tau$. Above this point $V_{1}(t)$ becomes independent of frequency.

Notice that for $\omega \tau \ll 1$, the phase angle $\psi$ approaches zero so that the signal voltage is practically in quadrature with the displacement from equilibrium of the vibrating plate. This is important for the purpose of making pd measurements during a retarding potential measurement. As it will be seen from the description of the physical setup, the vibrating probe will pick up an ac current from the electrons present between dynode and collector. The magnitude of this current can be orders of magnitude larger than the desired signal at the probe. It is the near quadrature of the two signals that allows a proper separation by the use of a synchronous detector. Anderson, Laponsky, Peria et al. ${ }^{74}$ at the University of Minnesota ran into this difficulty in their measurements of surface potential but did not resort to the near quadrature elimination, resulting in a considerable limitation in their measurements.

A field effect transistor preamplifier (voltage sensitive) was considered for picking up the signal $\mathrm{V}_{1}$ before going into a Princeton Applied Research synchronous 
detector. Initial calculations of $\mathrm{S} / \mathrm{N}$ ratio for the $\mathrm{FET}$ input for a current source with $C_{1} \simeq 5 \mathrm{pfd}, \mathrm{R}_{1} \simeq 10$ to $100 \mathrm{M} \Omega$, gave quite satisfactory results for operation up to $10 \mathrm{kc}$, with a half-power bandwidth of $6 \mathrm{kc}$ for a probe of $1 \mathrm{~mm}^{2}$ area, $\mathrm{d}=1 \mathrm{~mm}+0.05 \cos \omega \mathrm{t}$. At lower frequencies, where obtaining such oscillations would be easier but the noise level gets worse, $\mathrm{S} / \mathrm{N}$ power ratios of 50 seemed obtainable.

A simple calculation of the current needed to drive a speaker voice coil to an amplitude $\mathrm{A}$ gives

$$
i=\frac{m \omega^{2} A}{N 2 \pi r B}
$$

where $\mathrm{m}$ is the mass of the coil plus probe, $\omega$ is the angular frequency, $\mathrm{N}$ is the number of turns, $r$ is the radius of the voice coil, and $B$ is the field at the gap. For a J. B. Lansing Model LE-30 speaker these parameters are:

$$
\mathrm{N}=20, \mathrm{r}=2 \mathrm{~cm}, \mathrm{~B}=16,000 \text { gauss. }
$$

A maximum current of $1 \mathrm{amp}$ was calculated for easy dissipation in all aluminum voice coil forms by conduction through the "spider." For a total mass of 1 gram the amplitude vs. frequency is approximately

$$
A \simeq \frac{4 \times 10^{3}}{\omega^{2}}
$$

giving $.001 \mathrm{~mm}$ at $10 \mathrm{kc}$ and $.1 \mathrm{~mm}$ at $1 \mathrm{kc}$.

The approach seemed totally realizable for operation up to $1 \mathrm{kc}$ but one would have to rely on mechanical resonances of the driving system for operation at higher frequencies if required for faster risetime.

Initial tests were carried out with an inexpensive speaker driving a wooden rod with metallic tip. Results indicated the feasibility of the system, and pointed out the need for great care in shielding the probe tip from the voice coil, avoiding ground loops, and constructing the system with good rigidity to avoid undesirable mechanical resonances. 


\section{b. Retarding Potential Measurements With Planar Geometry}

The calculation of the energy distribution of emitted electrons with

a planar geometry has been reported by Schultz and Pomerantz. ${ }^{75}$ For N(V) being tht total number of secondaries emitted by the dynode but not reaching the collector, the energy distribution $G(V)$ is given by

$$
G(V)=-V\left(\frac{d^{2} N}{d V^{2}}\right)
$$

for a cosine distribution of emitted electrons. If the distribution is isotropic, the result is

$$
G(V)=-\frac{d N}{d V}-2 V\left(\frac{d^{2} N}{d V^{2}}\right) .
$$

For the measurement system being proposed, it appears necessary to operate the collector well grounded, with a small $1 \mathrm{~mm}^{2}$ section being the vibrating probe for pd measurements. The retarding potential is applied to the same wire from which the dymode current is measured. A typical I vs. V characteristic for a high gain dynode is shown on Fig. $32 . \mathrm{V}_{\mathrm{D}}$ is the dynode voltage, and for this particular illustration we assume that the c.p.d. is zero and the film is totally uncharged. For $\mathrm{V}_{\mathrm{D}}$ very negative, the dynode current $\mathrm{I}_{\mathrm{D}}$ is positive, indicating a net loss of electrons. With a primary beam of unity current, a fraction $\left(1-\eta_{t}\right)$ remains in the dynode, and a fraction $\left(\eta_{t}+\delta_{D}=\delta_{t}\right)$ leaves the dynode. In addition there is backscattering from the collector but we shall neglect this for the present.

As $V_{D}$ approaches zero from the negative side, secondaries from the collector due to the $\eta_{\mathrm{t}}$ transmitted primaries are more and more able to reach the dynode. At $\mathrm{V}_{\mathrm{D}}=0$, all secondaries from both sources reach their destinations. As $\mathrm{V}_{\mathrm{D}}$ is made positive, dynode electrons are being retarded until saturation. One can then measure dynode yield $\delta_{\mathrm{D}}$ including errors due to backscattering 


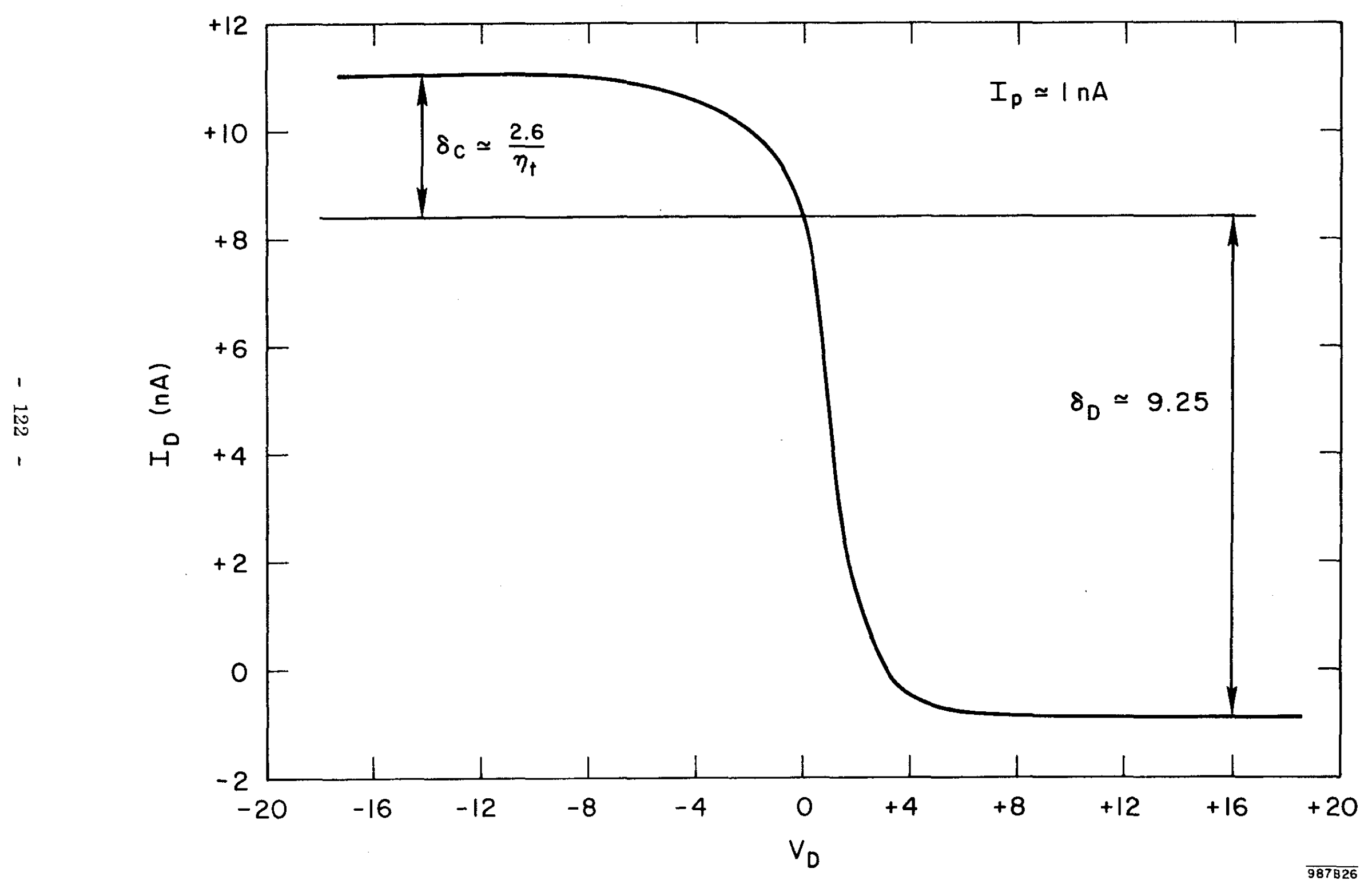

FIG. 32--Typical I vs. V characteristic for a high gain dynode 


$$
\frac{\mathrm{I}_{\mathrm{D}}\left(\mathrm{V}_{\mathrm{D}}=0\right)-\mathrm{I}_{\mathrm{D}}\left(\mathrm{V}_{\mathrm{D}}>+20\right)}{\mathrm{I}_{\mathrm{p}}}
$$

and also the effective yield of the collector to transmitted primaries, $\delta_{\mathrm{C}}$, as

$$
\frac{\mathrm{I}_{\mathrm{D}}\left(\mathrm{V}_{\mathrm{D}}<-20\right)-\mathrm{I}_{\mathrm{D}}\left(\mathrm{V}_{\mathrm{P}}=0\right)}{\eta_{\mathrm{t}} \mathrm{I}_{\mathrm{p}}} .
$$

If the angular distribution of secondaries were known to be exactly cosinelike, one could obtain the product $I \cdot\left(d^{2} I / d V^{2}\right)$ from the $V_{D}>0$ part of the I vs. $\mathrm{V}$ characteristic in order to find the energy distribution. For metals in reflection, it is well known that the secondaries have a near cosine angular distribution, ${ }^{5}$ but no data are available for alkali halides. Even in the absence of a significant surface barrier in the alkali halides, one can justify theoretically a roughly cosine distribution of emitted electrons if the internal distribution is isotropic. For electrons of a given energy $\mathcal{E}$ generated at some depth $d$ at an angle $\theta<90^{\circ}$, the path length for mostly forward scattering is roughly proportional to $d / \cos \theta$ and the probability of escape should be roughly inversely proportional to the path length.

In order to see how much error would be made by assuming an exact cosine distribution in the analysis of results, a computer program has been written for obtaining the energy distribution from data like those of Fig. 32 and arbitrary angular distribution. The program is based on the work of Schultz and Pomerantz ${ }^{75}$ who show that the total number of secondaries emitted by the dynode, but not reaching the collector, is

$$
N(V)=\int_{0}^{V} G(T) d T+\int_{V}^{\infty} R G(T) d T
$$

where $G$ is the energy distribution of secondaries, and $R=1-S$ is the 
fraction of electrons not reaching the shield. $S$ is given as

$$
S=\int_{0}^{\theta} f(\theta) \sin \theta d \theta / \int_{0}^{\pi / 2} f(\theta) \sin \theta d \theta
$$

where $\theta_{c}$ is the maximum angle of emission for which an electron with kinetic energy $\mathrm{T}$ will be collected when the retarding potential is $\mathrm{V}$, given by

$$
\theta_{\mathrm{c}}=\cos ^{-1}(\mathrm{~V} / \mathrm{T})^{1 / 2}, \quad \mathrm{~V} \leq \mathrm{T}
$$

The solution for $G(T)$ given an experimental $N(V)$ and an arbitrary angular distribution $f(\theta)$ can now be obtained by the following numerical method:

If one divides the range of energies and retarding potentials into steps $\mathrm{T}_{1}, \mathrm{~T}_{2}, \mathrm{~T}_{3}, \ldots, \mathrm{V}_{1}, \mathrm{~V}_{2}, \mathrm{~V}_{3}, \ldots$, it is possible to calculate the values of $\mathrm{S}\left(\mathrm{V}_{\mathrm{i}}, \mathrm{T}_{\mathrm{j}}\right)$ by carrying out the integrations of Eq. (24) with the limit $\theta_{\mathrm{c}}(\mathrm{i} / \mathrm{j})$ given by Eq. (25). From the values of $S$ one can form $R\left(V_{i}, T_{j}\right)$ and rewrite Eq. (23) in a matrix form by changing the integrals into summations as shown below. Let $N\left(V_{i}\right)=N_{i}, G\left(T_{j}\right)=G_{j}$, and $R\left(V_{i}, T_{j}\right)=R_{i j}$. Then

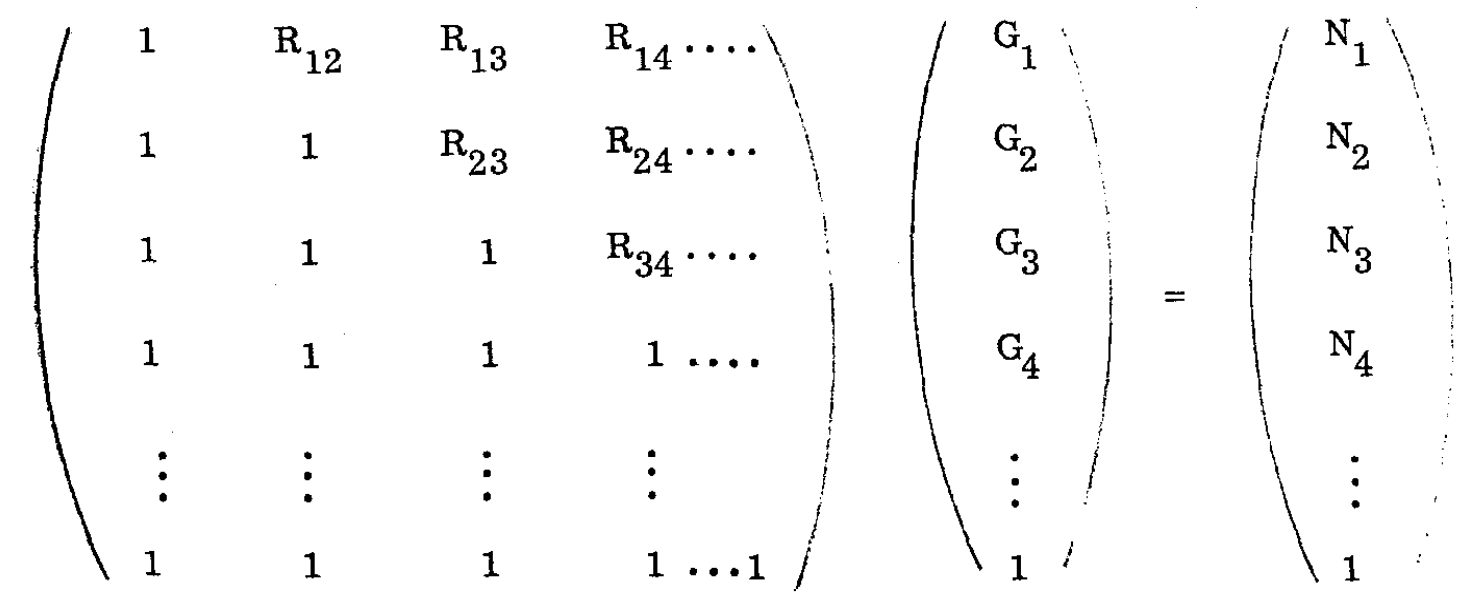

This forms a system of linear equations which can be solved for G. In practice, a range of 10 volts is taken in .1-volt steps for both $T$ and $V$ and the solution is obtained in a least squares sense. 
The results obtained for six different assumed angular distributions, starting from actual experimental data similar to the ones of Fig. 32 are shown in Fig. 33 (a), (b), and (c). Part (a) shows the results of the computation for an exact cosine distribution and a curve fitted through the points by eye. The scatter in points is very reasonable, as one is in effect taking a second derivative of the experimental results in the disguise of solving a set of equations. Part (b) of Fig. 33 shows a comparison of the best three lines for two quite substantial deviations from a cosine distribution and for the exact cosine, all normalized to unity area. Part (c) shows very extreme deviations from the cosine distribution. In particular, very large changes have been introduced at large angles. The results show that, except in the very extreme cases of Fig. $33(\mathrm{c})$, even quite substantial deviations from the cosine distribution will give results which are not too different from each other, and almost within the resolution of the system. As a conclusion, it seems then quite appropriate to assume a cosine distribution and obtain the secondary energy distribution from the experimental results by means of the computer program discussed. Alternatively, one could differentiate the curves electronically one time and smooth out the data before a second differentiation by computer. This has been tried and it turns out about as "noisy" as the process indicated above and it requires more steps.

It is interesting to look at Eq. (22) a bit further in relation with I vs. V characteristics for planar geometry like the one of Fig. 23 and Fig. 32. Secondary electron energy distributions from all materials have a general appearance like that of Fig.33(a). For metals the peak may be quite broad and have a center at several $\mathrm{eV}$, and for some alkali halides it seems to be narrower and lie below $1 \mathrm{eV}$, but there is one peak only and the curve $G(V)$ goes to zero at $V=0$. Examination of Eq. (22) shows that, for planar geometry, $\mathrm{dN} / \mathrm{dV}$ cannot have a peak except at $\mathrm{V}=0$. If there were one at some other value of $V$, there would be a zero in 


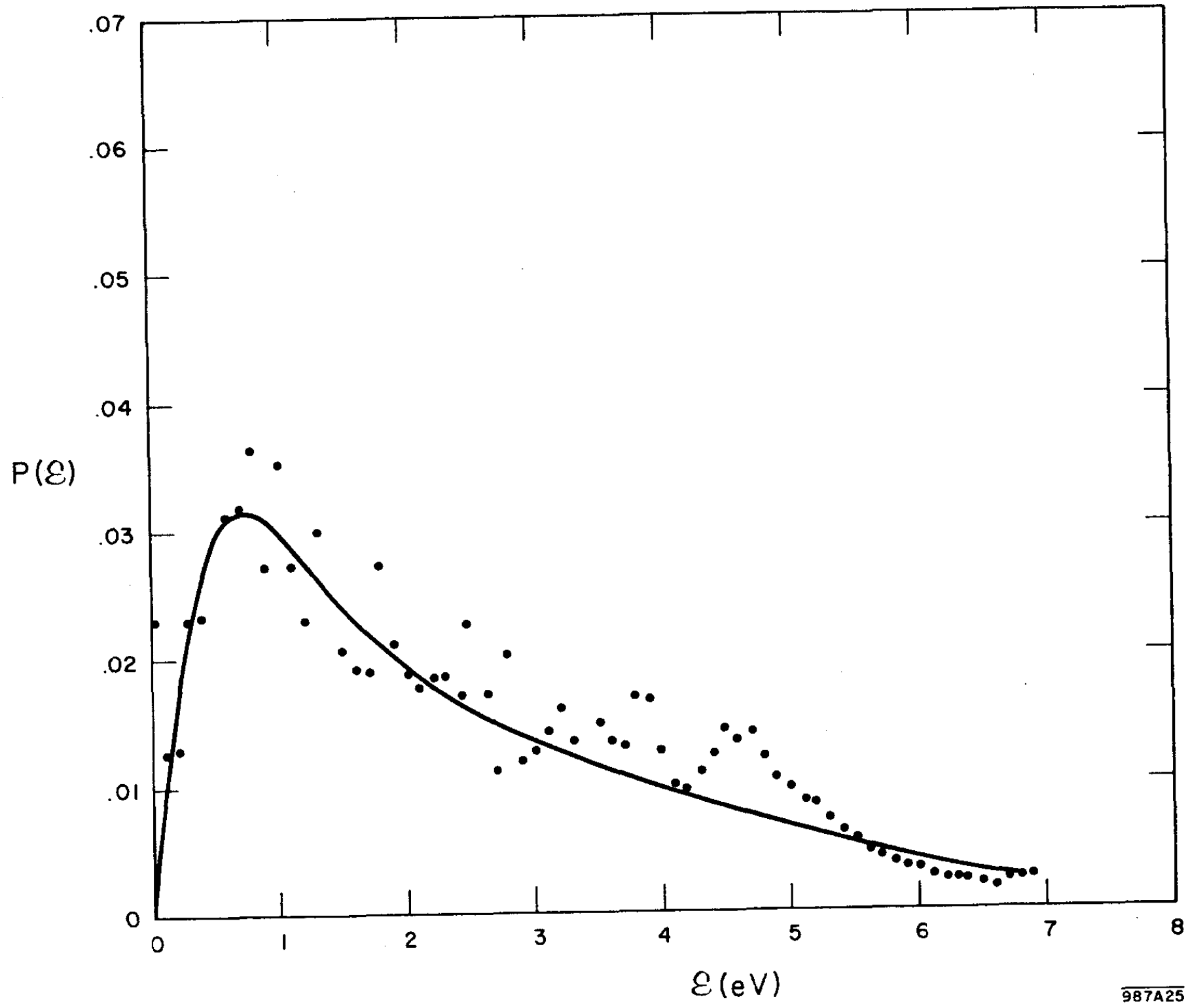

FIG. 33--Results of computing energy distributions for planar geometry

(a) Showing the spread in computed distribution points; from cosine distribution 


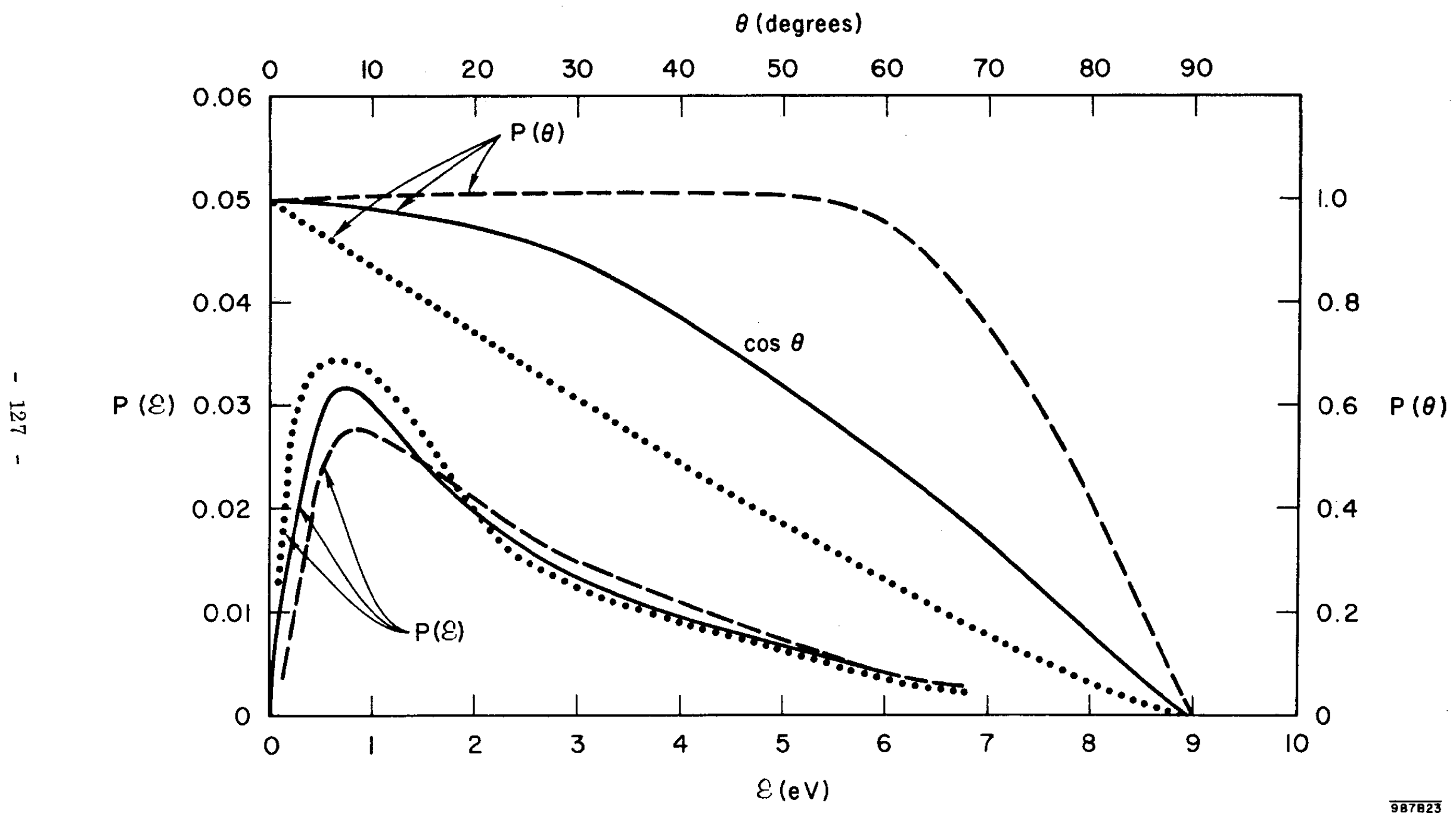

FIG. 33--Results of computing energy distributions for planar geometry

(b) For substantial deviations from cosine distributions 


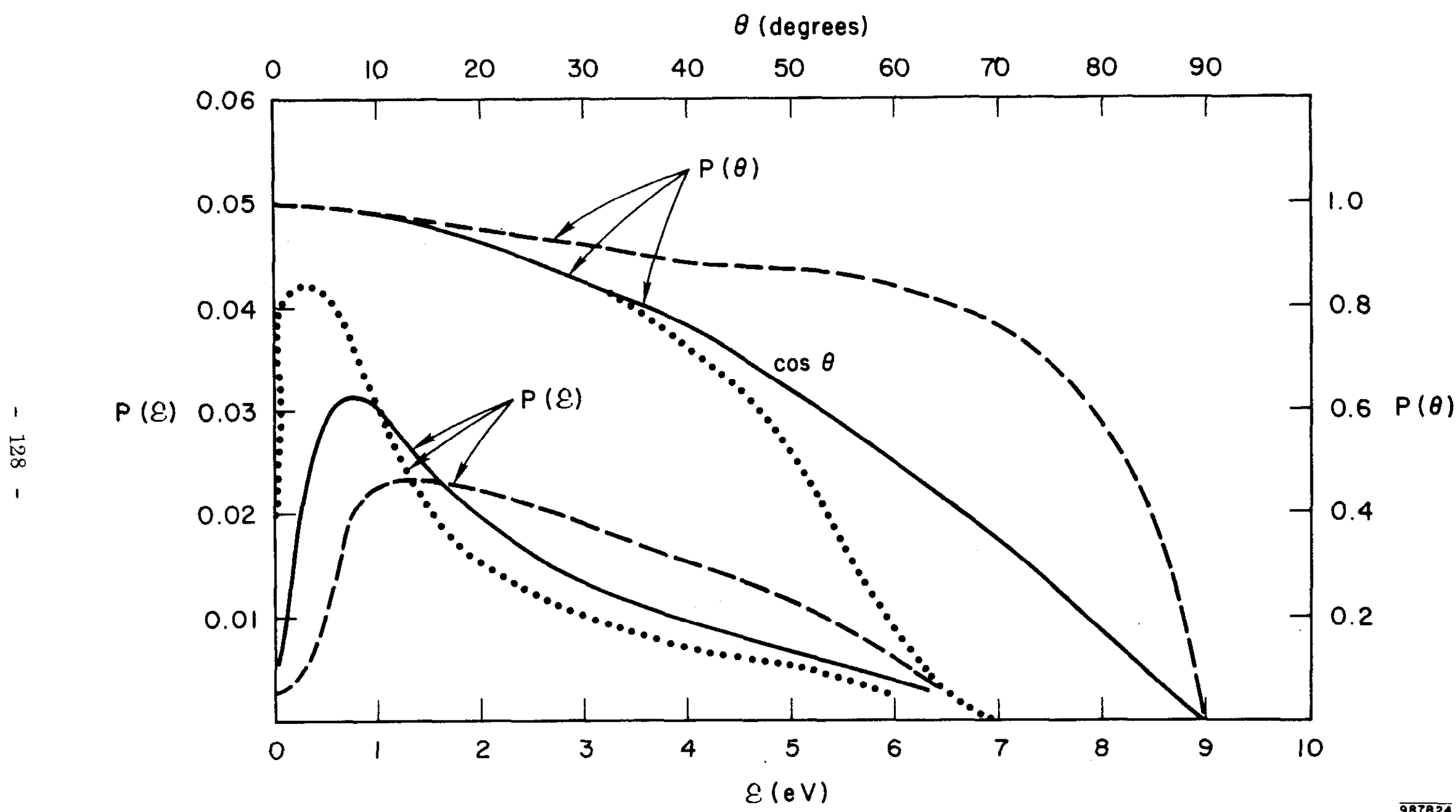

FIG. 33--Results of computing energy distributions for planar geometry

(c) For extreme deviations from cosine distributions 
the energy distribution curve at that $V$, contrary to findings. If one looks at a retarding potential measurement with electrons from two sources (for example, dynode and collector), it is clear that the above reasoning holds for both halves of the measurement and $\mathrm{V}=0$ corresponds necessarily to the point of maximum slope in the curve. This holds exactly for cosine distributions, but a computer analysis of what the I vs. V characteristics would look like for other distributions which are only near cosine-like, still supports the above conclusion. This fact can be useful in determining or checking a true zero cpd between two surfaces as was done in Section B2a of this chapter.

\section{Description of Equipment}

a. The Kelvin Probe Tube

Figure 34 shows a schematic drawing of the tube containing the Kelvin probe. The electron gun, identical to the one described previously, consists of a filament (1), which in this case is a $\mathrm{W}$ flat ribbon for a maximum current of $10 \mathrm{~A}$, a control electrode (2), and a grounded anode (3) with an opening of $1 / 4$ inch. The gun is normally operated in a defocused manner and the beam size is determined by the exit opening of the drift tube (4) which has a cross section of approximately $1 \mathrm{~cm}^{2}$. The drift tube is operated at +90 volts in order to prevent the emission of secondaries. A $95 \%$ open grid $G_{0}(5)$ is placed before the dynode (6) and is operated at $-300 \mathrm{~V}$, preventing the escape of secondaries from the back of the dynode. The $\mathrm{G}_{0}$ and dynode assembly is mounted on a pantograph (7) which can be manipulated from outside the tube with a micrometer, allowing accurate setting of the dynode to collector spacing "d." Not shown in the drawing is a mechanism allowing the lifting of the dynode from its centered position by about $2 \mathrm{~cm}$ independent of "d." The dynode and its holder can be removed from its location and replaced by lifting it up and out of the tube with a threaded rod through a $2-1 / 2$-inch i.d. flange not shown. The collector is an aluminum plate 


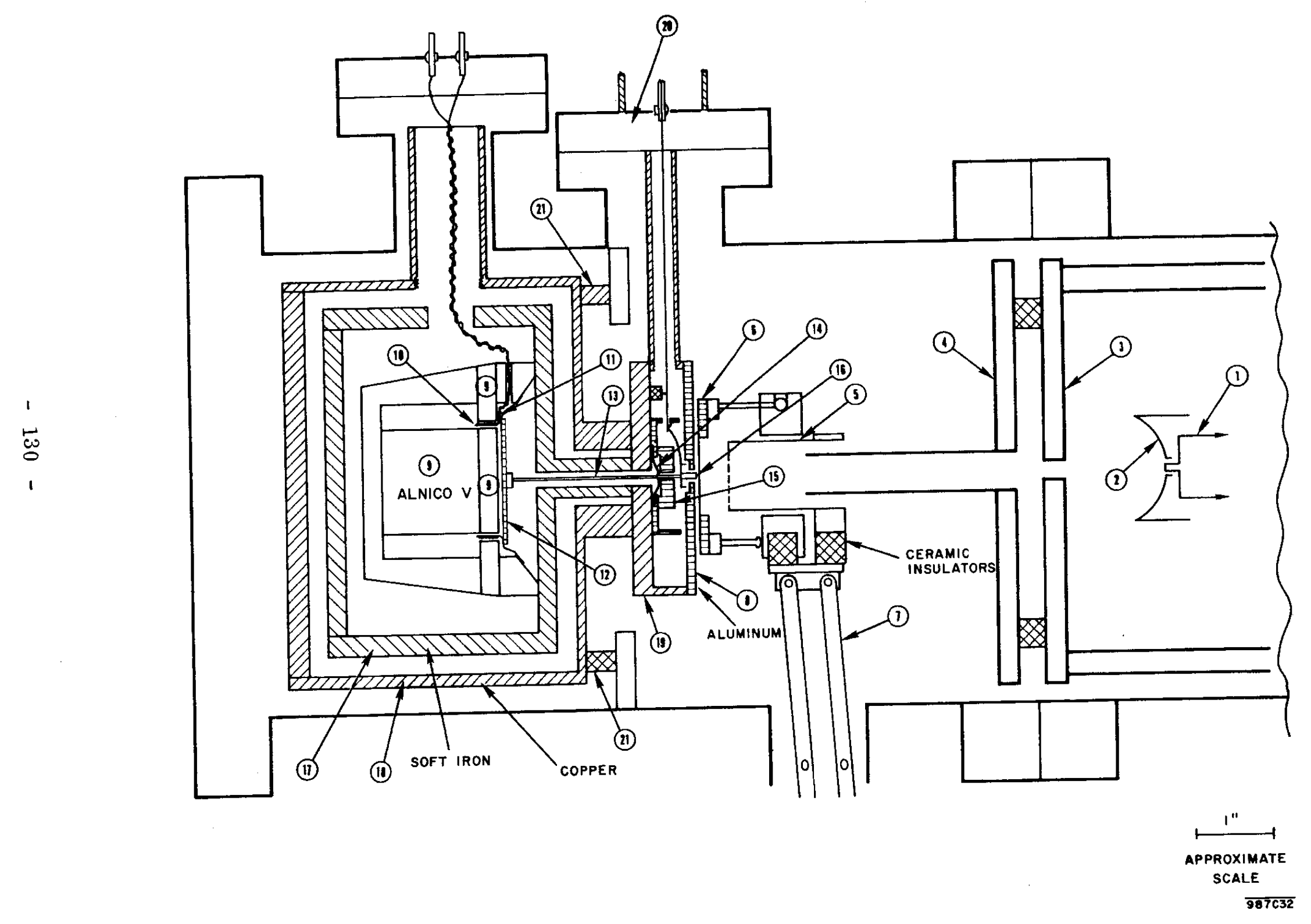

FIG. 34--Schematic drawing of the Kelvin probe tube 
$1 / 8$-inch thick with a hole in its center slightly larger than $1 \mathrm{~mm}^{2}$ to accomodate the vibrating tip (16) which has an area just under $1 \mathrm{~mm}^{2}$. The permanent magnet and pole pieces (9) with their housing came originally from a J. B. Lansing Model LE-30 Hi-Fi speaker. The parts were disassembled, stripped of paints, and cleaned up for high vacuum before reassembling and remagnetizing the Alnico $\mathrm{V}$ permanent magnet. The air gap was opened to .015 inch, with a measured magnetic field of near 16,000 gauss. The voice coil (10) was drawn from . 005-inch hard-drawn aluminum sheet and spot-welded to a six-legged "spider" (11) cut and shaped from the same material by means of a rubber sheet pressing against a steel dye. The coil-winding consists of 20 turns of .005 inch Al wire, with a ceramic "Duroc" coating. A very light coating of "Sauereisen" ceramic coating was used to hold the winding together. The coil wires were taken directly to a feed-through, keeping the voice coil well insulated from ground on both sides. An aluminum bar with $1.5 \mathrm{~mm}^{2}$ cross section (12) spot-welded to the voice coil is the support for a quartz rod (13) of approximately . 020-inch diameter with a second three-legged spider (14) glued to the rod with a drop of "Sauereisen" cement. The probe tip (16) is partly hollow in order to fit it to the quartz rod (glued with the same cement) and in conjunction with the machining of the collector plate forms a labyrinth to prevent electrons from penetrating the probe assembly. The aluminum shield (15) has been designed in such a way that any penetrating electrons cannot easily reach the insulator rod or the post for support of the electrical connection. If some charging still occurs, the shield also prevents the small piece of thin Al wire, which connects the probe tip to a rigid stainless steel rigid wire, from "seeing" the charged insulator to a very large extent. This wire, with its own vibrational modes, is made to see only aluminum from practically all directions. A $1 / 4$-inch thick nickel-plated soft iron shell (17) forms a shield against leakage of static magnetic fields and a 1/8-inch silver-plated copper shell (18) provides electrical 
shielding between the voice coil and the probe tip. The $1 / 4$-inch-thick base plate (19) is the common ground point for the assembly which is transmitted to the outside at the flange (20) through a copper tubing shielding the probe tip connection. Insulators (21) support the structure to the outer stainless steel shell. Vacuum connections are made to a Varian "VacSorb" pump for roughing and to a watercooled $11 \mathrm{l} / \mathrm{sec}$ Varian ion pump as a final stage. After the first baking for 48 hours at $125^{\circ} \mathrm{C}$, a final pressure of approximately $2 \times 10^{-8}$ torr is obtained. Baking to higher temperature results in damage to the voice coil and to some reduction in the magnetic field at the gap. With the system only vented to dry $\mathrm{N}_{2}$, the terminal pressure is obtainable in about 8 hours without further baking. The ion pump is about one foot removed from the tube and duly enclosed in magnetic shielding.

\section{b. The Electronic System}

The design of the electronic system supporting the Kelvin probe system has been carried out in such a way that measurements from very slow to moderately fast speeds can be made, the choice depending on the desired resolution and required speed in the retarding potential measurements to prevent charging. Three main circuits forming the system will be described.

(1) Regulated primary beam power supply

For measurement times of the order of one minute or more, in which the primary current may be required to be constant, a defocused, directly-heated cathode will not deliver anything near a constant current, even with perfect line regulation, due to long thermal time constants near the filament. To overcome this difficulty, the filament power supply has been designed with a simple SCR regulator at the ac line side. The feedback signal is taken from grid $\mathrm{G}_{0}$ in the Kelvin probe tube, suitably amplified. Fig. 35 (a) and (b) shows a simplified schematic of the power supply. The accelerating potential is applied 


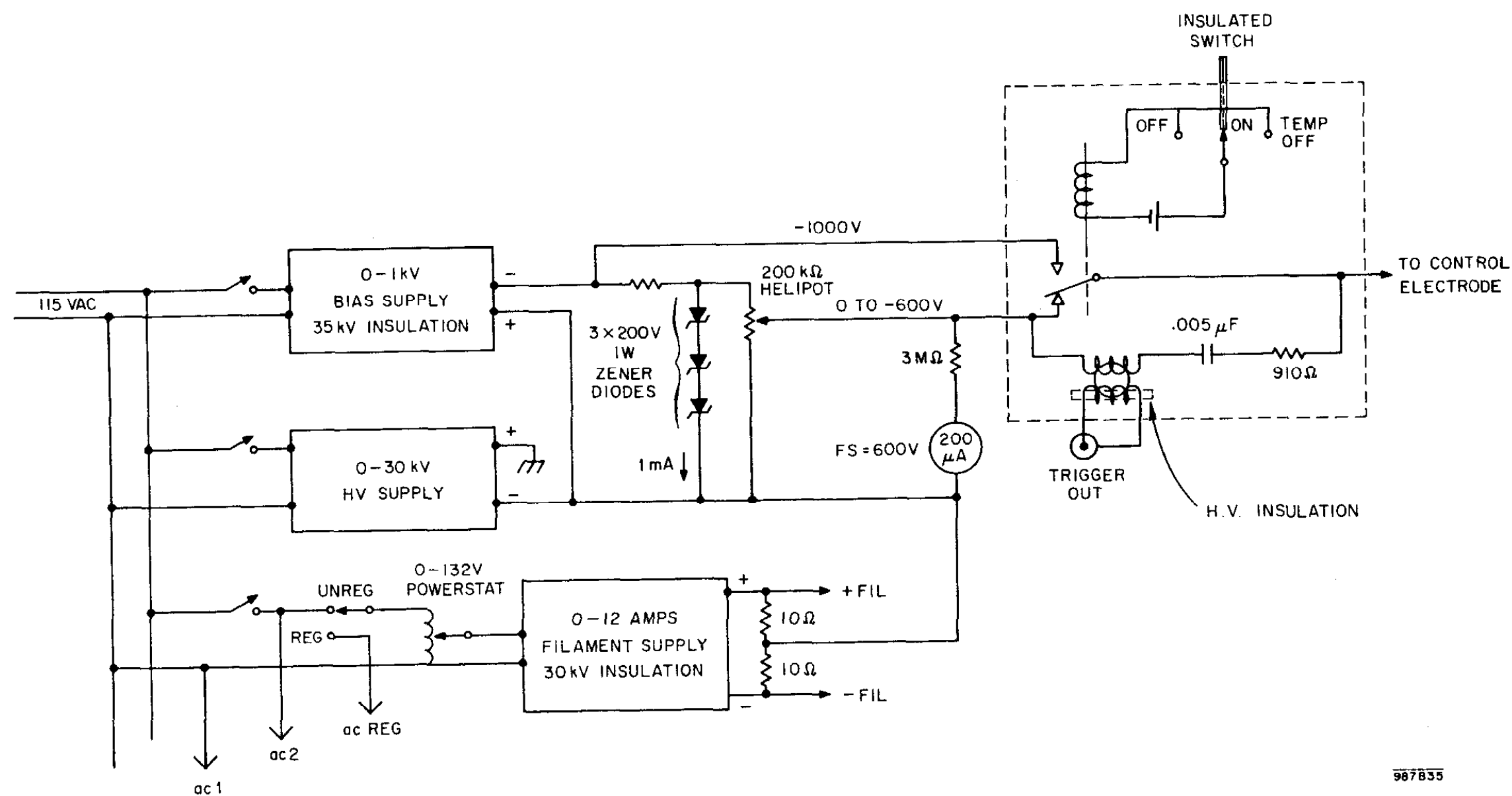

FIG. 35(a)--Schematic diagram of regulated primary beam power supply 


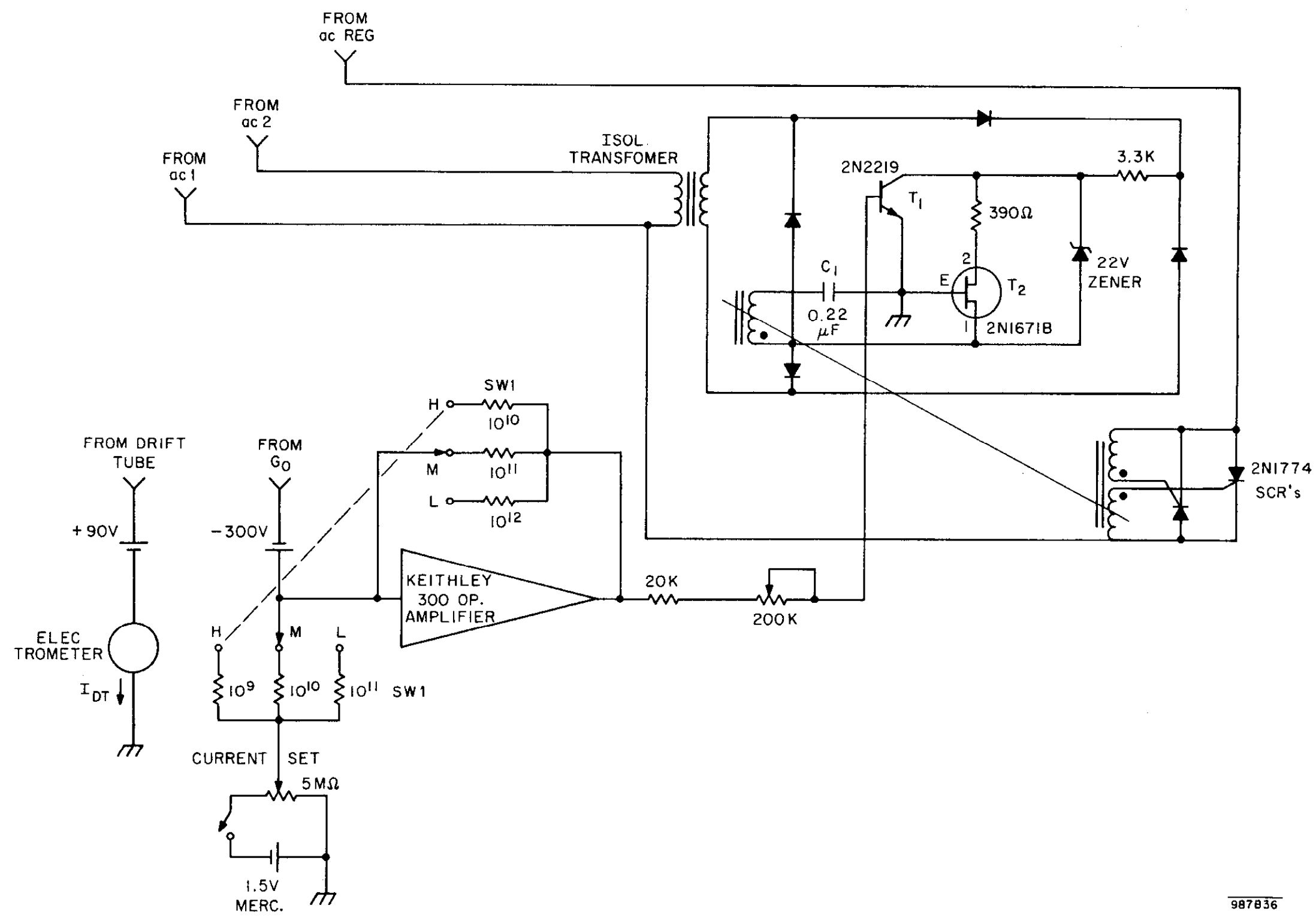

FIG. $35(\mathrm{~b})$--Schematic diagram of regulated primary beam power supply 
to the tube filament. The filament-to-control electrode potential is obtained from a Zener regulated 600 volt supply. A manually operated switch energizing a reed relay allows switching of the control electrode potential from its regulated setting to -1000 volts approximately, and back, providing a moderately fast way of turning on the tube. The risetime of the signal at the electrode has been measured to be $1 \mathrm{msec}$ or less. A trigger signal is also obtained at beam turn-on or turn-off. The current from $G_{0}$, suitably amplified, controls the charging current for capacitor $\mathrm{C}_{1}$ through transistor $\mathrm{T}_{1}$. As each half cycle of the ac line voltage starts, $\mathrm{C}_{1}$ starts charging at a basically constant current until the firing voltage for the unijunction transistor $T_{2}$ is reached. At that point, one of the SCR's will fire, allowing current to flow into the power transformer of the filament supply. This is a standard circuit technique for ac power regulation (General Electric SCR Manual). The desired primary current is set by the "Current Set" potentiometer and the position of swith SWI. The settings depend, of course, on the energy of the primary electrons, as the secondary emission coefficient of $G_{0}$ is energy dependent,but this is not troublesome.

The performance of the regulator is good for its intended purpose. A Nyquist analysis shows a maximum open loop gain of 50 at $\mathrm{dc}$, if stable operation is to be expected. The open loop gain at $5 \mathrm{cps}$ is only 5 under such conditions, indicating that short-term stability may not be as good as long-term stability. The long time constants of the power supply filtering, of the input circuit to the amplifier and of the filament itself result in the slow response of the loop.

In practice, it is possible to hold currents of .5 to $200 \mathrm{nA} / \mathrm{cm}^{2}$ within a few percent over long periods of time, with slightly larger variations at frequencies of a few cps.

When the beam has to be turned on for short periods of time with the reed relay switch, a nonregulated operation is provided. 


\section{(2) Dynode circuit}

The circuit for measuring the I vs. V characteristics of the dynode is shown in Fig. 36. A Hewlett-Packard function generator with the low side connected to a variable low-voltage supply provides a triangular waveform centered at some desired voltage $\mathrm{V}_{0}$. The lowest sweep frequency is . $01 \mathrm{cps}$. Dynode current is measured by a floating Keithley 602 electrometer in the "FAST" mode with risetimes of the order of $10 \mathrm{msec}$ in the range used. The I vs. V characteristic is plotted by a two-pen Mozeley 136A recorder,also with floating amplifier inputs. The three axes of the recorder track sine waves faithfully up to frequencies of $1 \mathrm{cps}$. If this is too slow for a particular measurement of a material, a storage oscilloscope with a differential input and good common mode rejection can be substituted. In carrying out a retarding potential measurement as described, the electrometer will register a step of current due to the differentiation of the ramp by the dynode-to-collector capacitance. In order to remove this step, a second signal is obtained from the H-P ramp generator which supplies this capacitive current through a 50 pfd capacitor. The response of the whole system to a current source between the dynode and collector (ground) corresponds to that of a low pass filter with a time constant of approximately $10^{-4} \mathrm{sec}$ with the electrometer in the "fast" mode.

(3) Kelvin probe circuit

The internally generated reference signal of a PAR Lock-In Amplifier, Model $\mathrm{HR}-8$, is used to drive a "Hi-Fi" power amplifier with heavier feedback than in the original design, for improved gain stability. The shielded output leads are taken to the voice coil feedthrough in the tube. One of the leads is grounded at the amplifier side, but its effect on stray signal pickup through ground loops seems to be negligible. The probe signal is picked up by a modified TMC-327A FET preamplifier and taken to the input of the PAR preamplifier. A series 


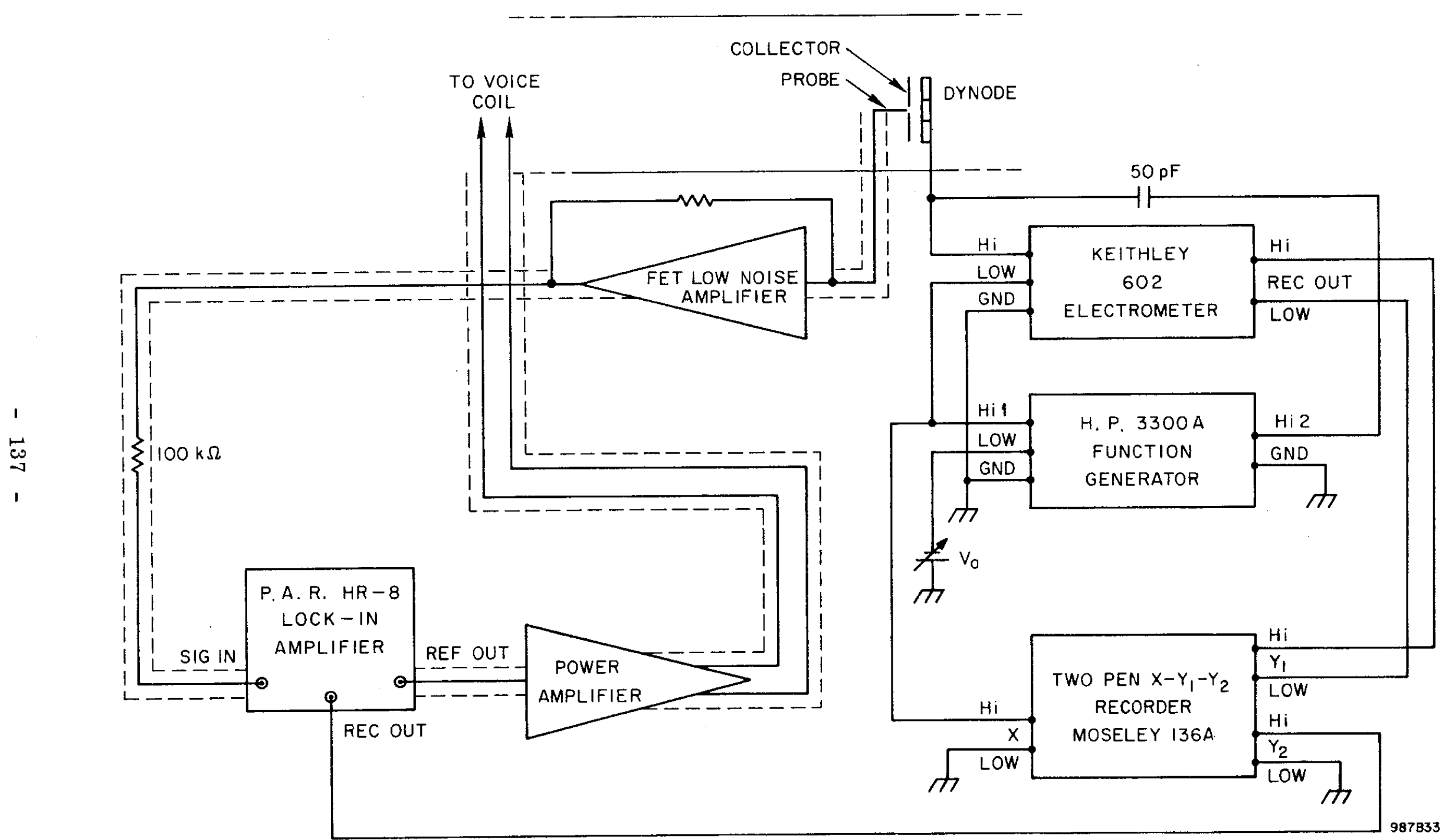

FIG. 36--Schematic diagram of dynode and Kelvin probe circuits 
resistor of $100 \mathrm{k} \Omega$ has been placed in the circuit to provide optimum noise performance from the PAR which otherwise adds noticeably to the noise of the FET preamplifier. The recorder output is taken to the second vertical channel of the two-pen recorder. This electronic loop is capable of working at any frequency within the audio range. A storage $x-y$ oscilloscope can be used for recording as well.

\section{Calibration and Initial Measurements}

This tube is not intended to produce an accurate measurement of secondary emission coefficient $\delta$. The lack of a grid between the dynode and the collector prevents a good measurement of $\eta_{t}$ and the planar geometry insures substantial errors in $\delta_{t}$ due to backscattering. Therefore, only approximate calibration of the primary current $I_{p}$ is necessary. Some care has been taken, however, with the uniformity of the beam at the dynode position. By placing a plate with a $\mathrm{ZnS}$ coating and with a $1-\mathrm{mm}^{2}$ hole at the center at the dynode holder, the beam profile is made visible from outside the tube through a flange fitted with a glass window. Also the current passing through the hole is picked up by the probe tip and can be measured. For primary energies between 4 and $12 \mathrm{keV}$, proper control electrode potentials were found giving a uniform illumination at the $\mathrm{ZnS}$ screen. Then by rotating a small magnet near the drift tube region, different sections of the beam were made to pass through the center hole. Current uniformity was measured to be $\pm 10 \%$ within a circle of half the beam radius.

The approximate primary current calibration has been carried out by placing a solid Al plate at the dynode position and measuring both the dynode current, $\mathrm{I}_{\mathrm{D}}$ ' and the drift tube current, $\mathrm{I}_{\mathrm{DT}}$. Assuming that about $15 \%$ of primary electrons are backscattered and no other currents play any significant role, a table of $\mathrm{I}_{\mathrm{DT}}$ vs. $E_{p}$ for a constant $I_{p}=10 \mathrm{nA}$ was formed. These currents $I_{D T}$ range between $-2.33 \mathrm{nA}$ at $\mathrm{E}_{\mathrm{p}}=4 \mathrm{keV}$, and $-1.23 \mathrm{nA}$ at $12 \mathrm{keV}$. Linearity of $I_{p}$ vs. $I_{D T}$ at fixed $E_{p}$ holds to $\pm 5 \%$ within 3 decades. 
The current $I_{D T}$ has been taken as a scale for $I_{p}$ because $I_{D T}$ is quite independent of the collector to dynode spacing $d$. Notice that changing $d$ also changes the $\mathrm{G}_{0}$-to-DT. spacing resulting in some dependence of $\mathrm{I}_{\mathrm{G}_{0}}$ on $\mathrm{d}$. These changes are not very small compared to $\mathrm{I}_{\mathrm{G}_{0}}$, but they are very small compared to $\mathrm{I}_{\mathrm{DT}}$. D.C. measurements of $\delta_{\mathrm{t}}$ from the simple formula

$$
\delta_{\mathrm{t}}=1-\frac{\mathrm{I}_{\mathrm{D}}}{\mathrm{I}_{\mathrm{p}}}
$$

or of $\delta$ by the method of Fig. 32 on standard substrates and on CsI films trans ferred in a dry $\mathrm{N}_{2}$ atmosphere consistently give high results, the error being quite explainable in terms of the backscattering difficulties and some inaccuracy in the values of $I_{p}$.

The Kelvin probe has been tested quite extensively in order to determine conditions for the best sensitivity, stability, etc. Since fast response of the probe is not of the most important concern for the measurements to be reported here, operation at approximately $500 \mathrm{cps}$ with a very good sensitivity has been adopted. With a drive of less than .1 watt into the voice coil and $\mathrm{d} \simeq 1 \mathrm{~mm}$, potential changes of .05 volts can be detected quite unequivocally with a $12 \mathrm{db} /$ octave filter time constant of $.1 \mathrm{sec}$. For less demanding requirements in sensitivity, operation at higher frequencies is quite possible, up to about $5 \mathrm{kc}$, but operation at the higher frequencies is tricky due to the presence of strong vibrational modes at the probe with small components of axial displacement. Deviations from linearity are not observable in dynode potentials ranging between -100 to $+100 \mathrm{~V}$, at different spacings $d$. By careful adjustment of the phase between the reference signal driving the probe and signal driving the phase sensitive detector at the PAR instrument, it is possible to render the probe quite insensitive to the presence of secondary and transmitted primaries at least up to currents of $100 \mu \mathrm{A}$. Reversible changes in cpd of less than . 05 volts have been observed a few times between the 
collector and a metallic dynode by turning the primary beam on and off at $I_{p}=50 \mu \mathrm{A}$. Whether this change in cpd is real, due to different surface conditions under electron bombardment, or is due to slight misadjustment of the phase control cannot be ascertained. In order to maintain a long-term phase adjustment it is necessary to keep the PAR instrument on all the time and,of course, not to change any of the controls which may affect phasing. Sensitivity of the amplifier stage may be changed, however, as well as the level of the reference drive signal.

In spite of the care taken with shieldings of different kinds, two minor difficulties still remain with the probe:(1) Even at a true zero cpd some signal is picked up by the probe tip. (2) Some charging still occurs inside the probe chamber when the electron beam is on. The first problem results in a constant but unknown zero offset. The second one results in a variable zero offset which changes in one direction to some final value at a very slow rate with the beam on, and returns to the uncharged condition over a period of days. Fortunately the rate of change is slow enough that it can be totally neglected in measurements with the electron beam at $\mathrm{I}_{\mathrm{p}}<10 \mu \mathrm{A} \mathrm{cm} \mathrm{cm}^{2}$ if a particular measurement can be completed in periods of a few minutes, which certainly is the case in practice.

The measurement of pd between collector and some other dynode surface is carried out in a way which is independent of the actual zero offset at the time of measurement, as long as it stays constant. With the collector-to-dynode distance $d$ at a given setting, $V_{D}$ is swept about zero to suitable voltages, for example, \pm 2.5 volts. The de output of the $\mathrm{PAR}$ instrument, $\mathrm{V}_{\mathrm{S}}$, is then plotted vs. $\mathrm{V}_{\mathrm{D}}$. Next the spacing $d$ is changed and a second sweep is made. There is a partic ular value of $V_{D}$ such that the two traces intersect. At that point the Kelvin probe finds a true zero pd as its output is independent of spacing $d$. If the crossing occurs at $V_{D}=V_{D 0}$, then the dynode surface has a potential $-V_{D 0}$ with respect to the collector metal. 
Figure 37 (a) shows the results of a cpd measurement, plotted on a storage oscilloscope, between a collector and probe tip covered with a $500 \AA$ layer of $\mathrm{Au}$ and a substrate formed by $1000 \AA \mathrm{Al}_{2} \mathrm{O}_{3}+500 \AA$ Au. The horizontal scale, $\mathrm{V}_{D^{\prime}}$, is $.5 \mathrm{volt} / \mathrm{cm}$ with a zero at center scale. The result is zero cpd to better than .05 volt. The electron beam was off. Part (b) shows a similar measurement between the $\mathrm{Au}$ collector and a standard $\mathrm{Al}_{2} \mathrm{O}_{3}+\mathrm{Al}$ substrate with the beam off. Part (c) corresponds to the same measurement with the beam on at $I_{p}=50 \mathrm{nA}$, $\mathrm{E}_{\mathrm{p}}=12 \mathrm{keV}$. Results for (b) and (c) show that $\mathrm{Al}$ is positive with respect to $\mathrm{Au}$ (with the surface conditions prevalent during the measurement) by .9 to .95 volt. After the Au layer in the collector and tip was removed, a cpd measurement on a gold dynode gave results of the same magnitude as those of Fig. 37(b) and (c), but of opposite sign. $\mathrm{Al}-\mathrm{Al}$ cpd is often found to be -.1 to -.2 volt (substrate with respect to collector) before electron irradiation, going towards zero irreversibly after bombardment for a few minutes with penetrating electrons.

\section{Measurements on Alkali Halide Films}

A substantial amount of effort has been devoted to finding a good way of making retarding potential measurements on alkali halide films while keeping the pd fixed at an essentially uncharged condition. From experiments with several films of $\mathrm{CsI}$ and $\mathrm{KCl}$ it is found that the potential at the surface of a composite film (with respect to the collector) ranges between $+.1 \mathrm{~V}$ and $-.1 \mathrm{~V}$, which is essentially the same as one finds for the contact potential of substrates alone. Thus it appears that the presence of the alkali halide layer does not substantially affect the work function of the Al backing when the film is new. The result is a bit different for the case of NaF. It has been found that $V_{D 0}$ is approximately -.5 volts. If the film is uncharged, then some dissociation may have occurred during evaporation with the result that $\mathrm{Na}$ ions may have lowered the work function of the Al backing. 


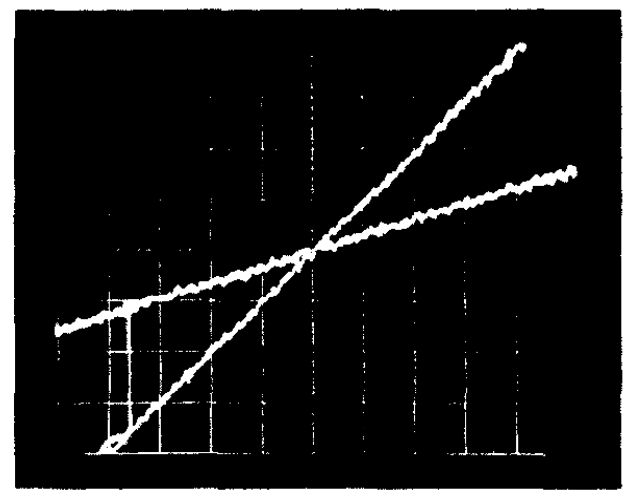

(a)

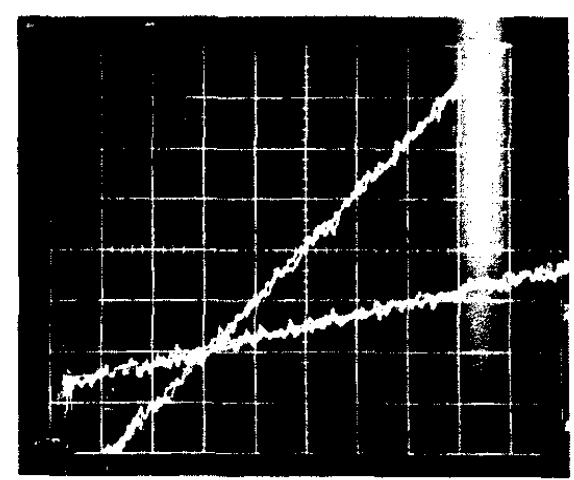

(b)

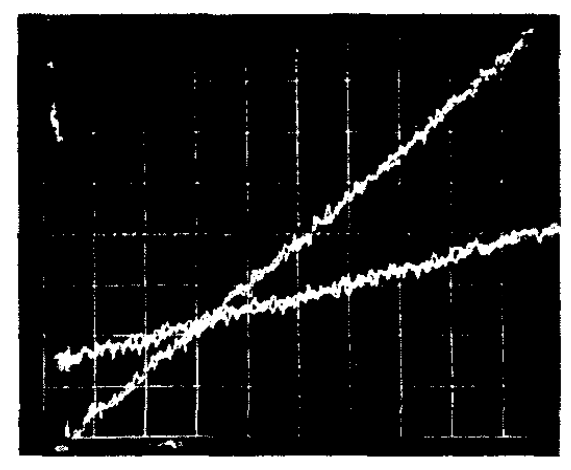

(c)

\section{$\frac{\text { COLLECTOR }}{A u-\frac{\text { DYNODE }}{A u}-\frac{{ }^{-}}{\text {BEAM OFF }}}$}

BEAM OFF

\section{$\mathrm{Au} \quad \mathrm{Al}$ \\ BEAM ON $12 \mathrm{KeV}, 50 \mathrm{~mA}$}


Under prolonged bombardment, CsI films show two different kinds of changes: (1) an irreversible change of $\mathrm{V}_{\mathrm{D} 0}$ (becoming more negative) which does not affect the I vs. $V_{D}$ characteristics of the film, except for a shift in position, and (2) a reversible change of $V_{D 0}$ in the same direction whose effect on the I vs. $V_{D}$ characteristics is indicative of patch positive charging of the dynode surface. This patch effect does not result in enhancement of secondary yield, however, The irreversible change, of up to about $1 \mathrm{eV}$, is linked to dissociation of the alkali halide with the iodine probably reacting chemically with the Al layer. The outline of the electron beam, including the shadow of a grid, are clearly visible on the substrate after exposure of the film to room air, even after washing the film away with water.

The nature of the charging mechanism has been studied only as far as necessary to learn how to avoid it, and a good explanation for some of the observed effects will not be attempted here.

The outcome of the investigation has been the finding of the following technique for making reliable retarding potential measurements:

1. After evaporation, the film is directly transferred to the Kelvin probe tube with exposure to dry nitrogen with less than $1.5 \%$ relative humidity.

2. After pumpdown to approximately $2 \times 10^{-8}$ torr, the first pd measurement is carried out by the standard method indicated above. With the beam cutoff, the filament is allowed to heat for about 5 minutes at a predetermined current which is known to result in a primary current of approximately $1 \mathrm{nA} / \mathrm{cm}^{2}$.

3. A retarding potential measurement for a desired $\mathrm{E}_{\mathrm{p}}$ is carried out by sweeping $V_{D}$ from -1 to +9 volts, and back, linearly in 10 seconds maximum. Only during that time is the beam turned on. 
4. A new pd measurement is made to check that no substantial charging has occurred, and the procedure is repeated for other $\mathrm{E}_{\mathrm{p}}$ 's .

It has also been determined that the secondary emission from the films due to electrons scattered back into the dynode surface from the collector (trans mitted and subsequently backscattered primaries) does not affect the energy distribution measurement. This has been ascertained by comparing the resulting energy distributions for the case of a collector covered with a $500 \mathrm{~A}$ layer of $\mathrm{Au}$ (high backscattering coefficient) and the case of an Al collector (low backscattering). No differences could be detected between the two cases.

For the four materials studied, three consecutive measurements have been made, one at $\mathrm{E}_{\mathrm{p}}=4$ or $5 \mathrm{keV}$ (point of low yield), one at $\mathrm{E}_{\mathrm{p}}=7$ or $8 \mathrm{keV}$ (highest yield), and the third one $12 \mathrm{keV}$ (maximum primary penetration). The thickness selected has been $500 \AA$ 列 insure isolation of effects due to the $\mathrm{Al}$ backing. It has been found that potential differences changed during the measurements by less than 0.05 volts, which would not affect the measurements in any substantial manner.

The results obtained have been analyzed by the method indicated in Section C1b of this report assuming a $\cos \theta$ angular distribution of emitted electrons. The results are shown in Figs. 38 to 41 . The calculations have been carried out at intervals of $.1 \mathrm{eV}$ and the results are normalized to unity area. It is immediately apparent that there is a substantial difference between the high $\mathrm{Z}$, medium $\mathrm{Z}$, and low $\mathrm{Z}$ alkali halides, and a ready explanation can be given in terms of the results of the electron-phonon interaction calculations. As one considers materials with decreasing $Z$, the escape probability of low energy electrons becomes very strongly reduced. Thus if one assumes some internal secondary generation function which extends from zero energy to near the conduction band energy, more or less monotonically decreasing with energy, the experimental results can be 


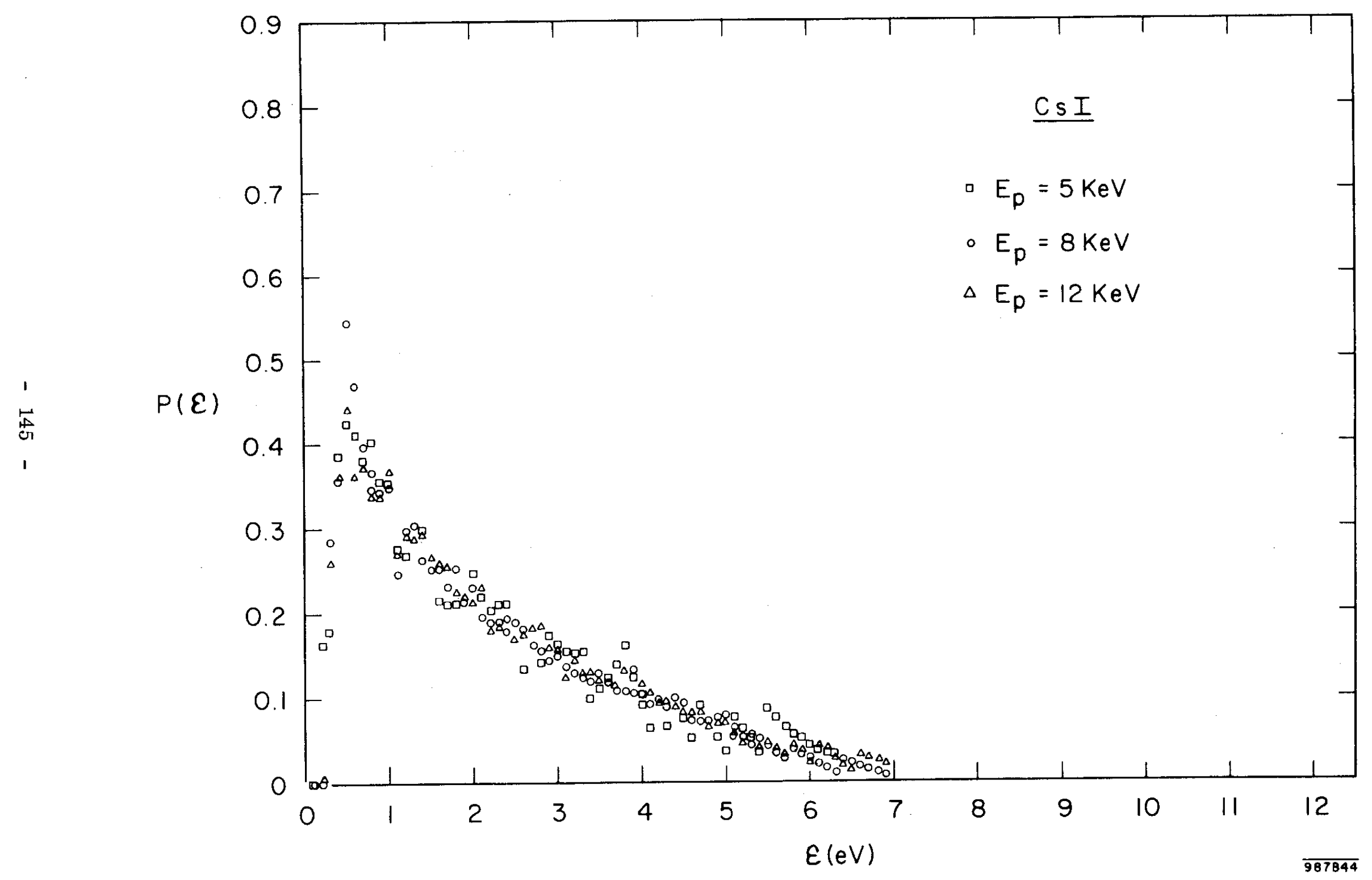

FIG. 38--Energy distribution of emitted secondary electrons from CsI films 


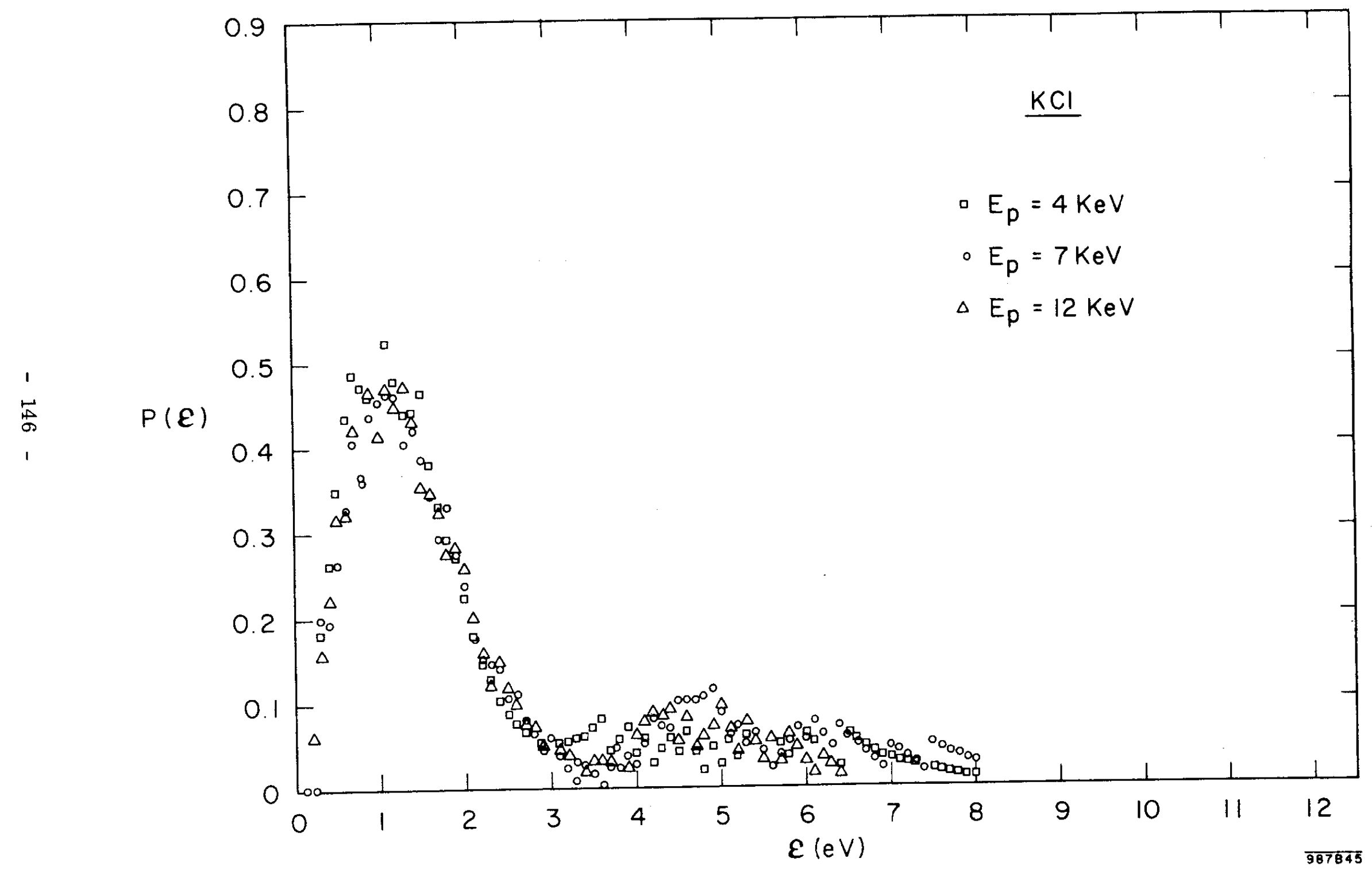

FIG. 39--Energy distribution of emitted secondary electrons from $\mathrm{KCl}$ films 


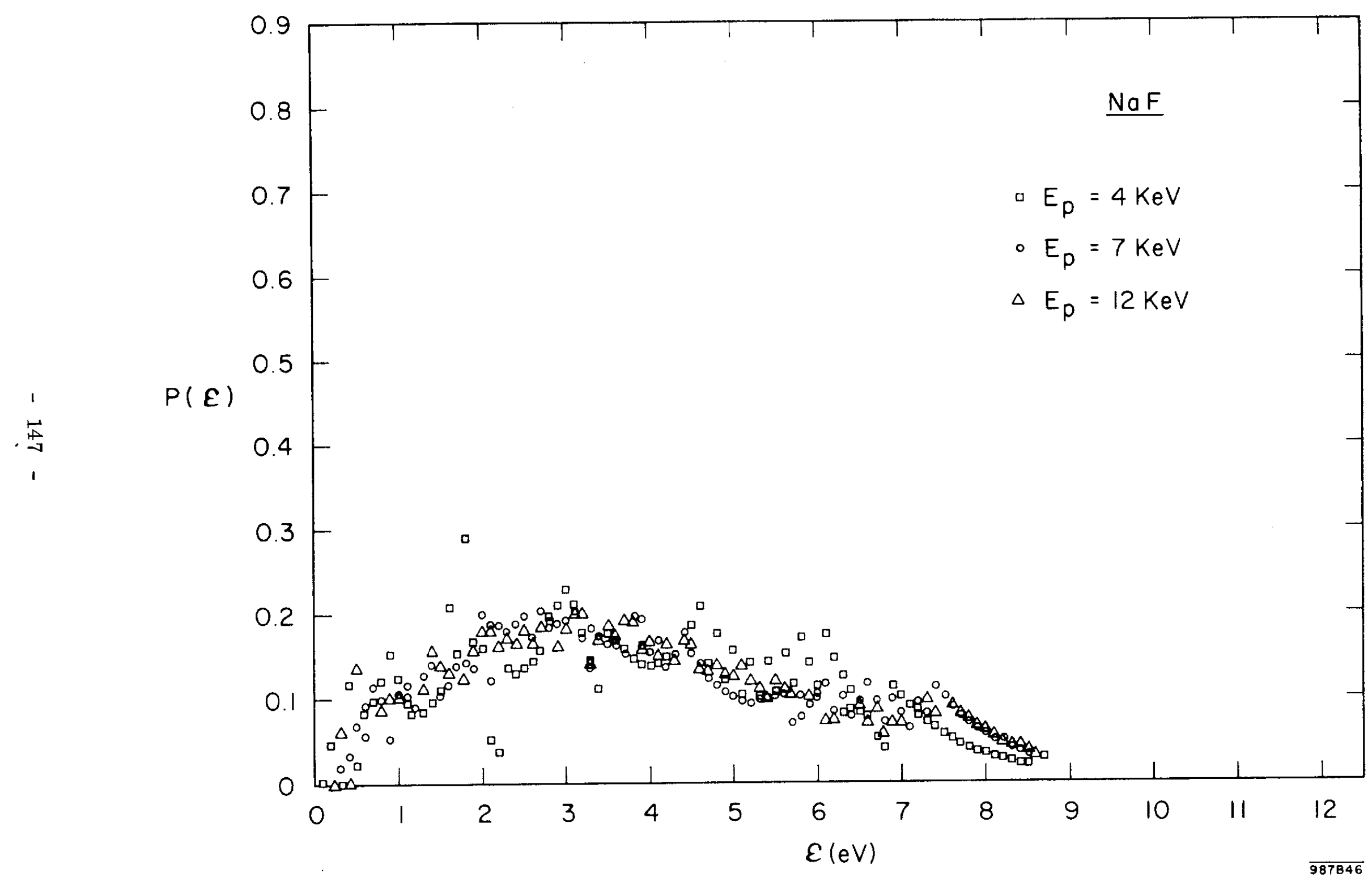

FIG. 40--Energy distribution of emitted secondary electrons from NaF films 


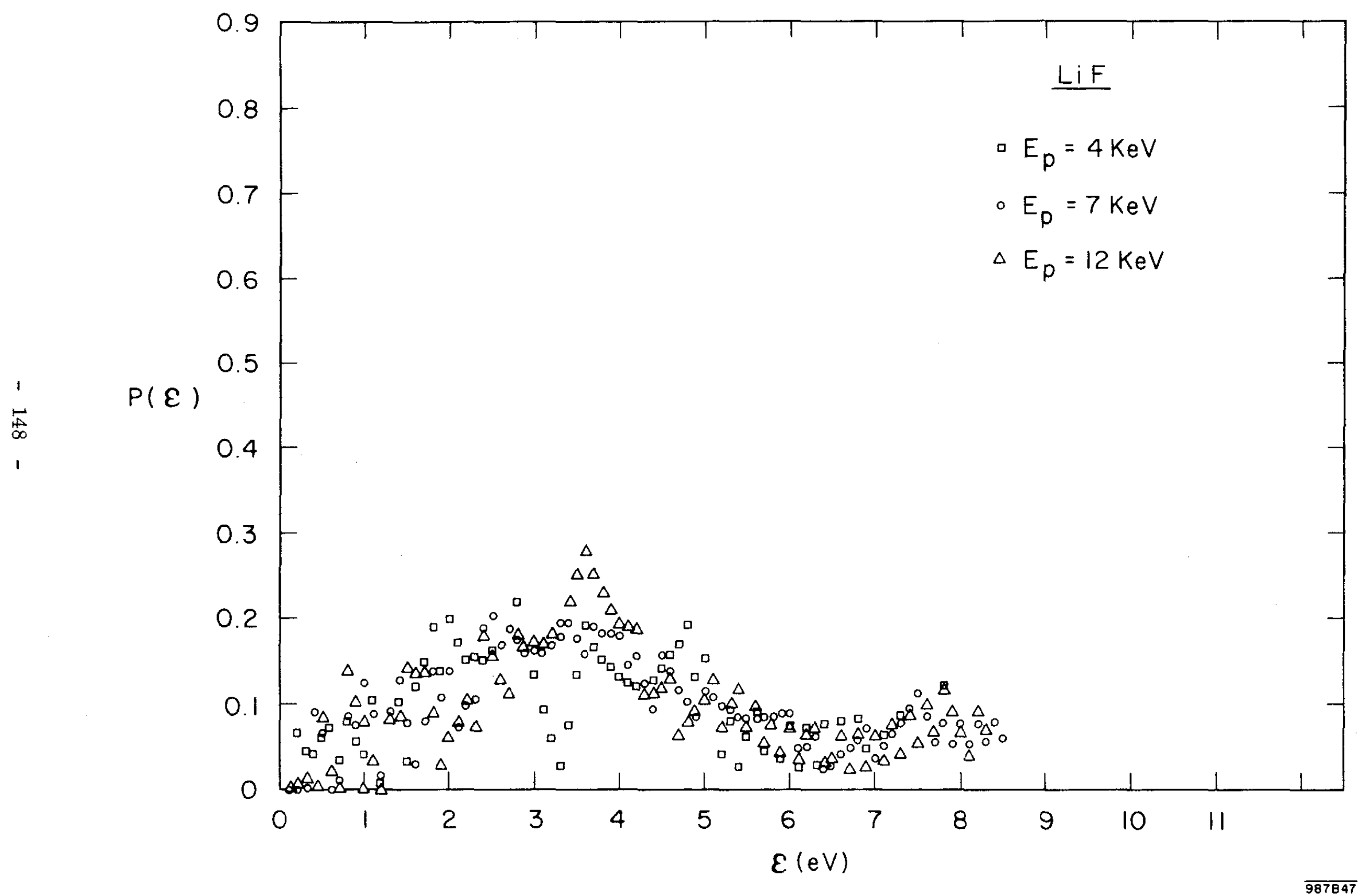

FIG. 41--Energy distribution of emitted secondary electrons from LiF films 
explained qualitatively. It is also interesting to note that the results for low, medium, and high $E_{p}$ cannot be differentiated from each other (for low $E_{p}$, the results are by necessity noisier). It would appear that at low $\mathrm{E}_{\mathrm{p}}$ there shouid be a lack of electrons with low escape probability, as the peak of $-d E / d x$ is expected to be located far from the exit surface. If one examines Figs. 4 and 5 for $500 \mathrm{~A}$ films of $\mathrm{CsI}$ and $\mathrm{KCl}$, it is clear that the above reasoning is wrong for $\mathrm{KCl}$ and would be also for the lighter materials. Some effect might be expected for CsI but in that material the escape length $\mathrm{L}_{\mathrm{S}}$ is quite high even for $.5 \mathrm{eV}$ electrons. Thus, for a $500 \AA$ film, the peak of $-\mathrm{dE} / \mathrm{dx}$ is near the exit surface of the film even at 4 to $5 \mathrm{keV}$, but the yield is low because the transmission of the substrate is low at those energies.

The energy distribution becomes very near zero for $\mathcal{G}=6$ to $7 \mathrm{eV}$ in $\mathrm{CsI}$, for $\mathcal{E}=7$ to $8 \mathrm{eV}$ in $\mathrm{KCl}$, and for $\mathcal{E}=8$ to $9 \mathrm{eV}$ in $\mathrm{NaF}$. For the case of $\mathrm{LiF}$, the decrease to zero occurs at even higher energy. The location of the first excitonic absorption edge for these materials is approximately $5.7,7.6,10.5$, and $11 \mathrm{eV}$, respectively. Thus the increase in band gap is reflected in the energy distribution of the emitted secondaries. It must be stated that the calculation of energy distribution at the high energy end is much less accurate than in the middle ranges, as results are very dependent on small changes in the $I_{D}$ vs $v_{D}$ characteristics under study (see Eq. 22). Therefore only qualitative statements can be made about the high energy part of Figs. 38 to 41 .

The appearance of dip near $\mathcal{G}=3.5 \mathrm{eV}$ in $\mathrm{KCl}$ (Fig. 39) is not an error. The experimental I vs. $V_{D}$ curves nearly have an inflection point at that energy. Some apparent sharp peaks at other points in all the graphs are, however, due to the method of analysis and have no physical significance. 


\section{QUANTITATIVE ANAL YSIS OF RESULTS}

A. The Model for Transmission Secondary Emission

1. Mathematical Model

In Chapter I of this report, the generation of internal secondary electrons in alkali halide films was studied. It was proposed that there exists a function $G$ for a given material such that $G\left(E_{0}\right) d E_{0}$ is the number of low energy electrons generated between energies $\mathcal{E}_{0}$ and $\mathcal{E}_{0}+\mathrm{d} \mathcal{E}_{0}$ per unit energy loss suffered by the primary beam at a particular point in the film. The function $G$ is assumed independent of primary energy, of $x$, and to have an isotropic angular distribution of velocities.

Then for a given film and $\mathrm{E}_{\mathrm{p}}$, the production of internal secondaries between energies $\mathcal{E}_{0}$ and $\mathcal{E}_{0}+\mathrm{d} \mathcal{E}_{0}$ in a slab of thickness $\mathrm{dx}$ at $\mathrm{x}^{\prime}$ is given by

$$
\left(-\frac{\mathrm{dE}}{\mathrm{dx}}\left(\mathrm{x}^{\prime}\right)\right) \mathrm{G}\left(\mathscr{E}_{0}\right) \mathrm{d} \mathscr{E}_{0} \mathrm{dx} .
$$

The escape probability of the internal secondary electrons as governed by the electron-phonon interaction was also studied in Chapter I and found to be very closely exponential in character. An escape depth $L_{S}\left(\mathcal{E}_{0}\right)$ dependent on $\mathcal{G}_{0}$, the initial energy of the electron, and on the material, was defined for an initially isotropic distribution of velocities. Likewise, a constant $\mathrm{P}_{0}\left(\mathcal{E}_{0}\right)$ corresponding to the probability of escape of electrons generated isotropically at the film exit surface was given.

Assuming that the transmission secondary yield of uncharged alkali halide films is not governed by any mechanism beyond the two just mentioned, the secondary yield $\delta$ should be obtainable from an expression

$$
\begin{gathered}
\delta=\int_{\mathrm{x}} \int_{\xi_{0}} \mathrm{G}\left(\xi_{0}\right)\left(-\frac{\mathrm{dE}}{\mathrm{dx}}(\mathrm{x})\right) \mathrm{P}_{0}\left(\xi_{0}\right) \exp \left(-\frac{\tau-\mathrm{x}}{\mathrm{L}_{\mathrm{S}}\left(\xi_{0}\right)}\right) \mathrm{d} \xi_{0} \mathrm{dx} \\
-150-
\end{gathered}
$$


where the integral over $\mathrm{x}$ runs over the film thickness and the integral over $\mathcal{E}_{0}$ should cover the range of energies of internally generated electrons. Since the present calculations of energy loss and escape probabilities have been made for discrete values of $x$ and $\mathscr{E}_{0}$, an approximation can be made to the above equation by writing

$$
\delta=\sum_{\mathscr{E}_{0 j}} G\left(\mathscr{G}_{0 j}\right) P_{0}\left(\mathscr{G}_{0 j}\right)\left\{\sum_{x_{i}}\left(-\frac{d E}{d x}\left(x_{i}\right)\right) \exp \left[-\frac{\tau-x_{i}}{L_{s}\left(\varepsilon_{0 j}\right)}\right] \Delta x\right\} \Delta E_{0 j} .
$$

For a given $\mathscr{E}_{0 j}$ the summation over $x_{i}$ can be carried out by using the results obtained in Chapter I for $\mathrm{dE} / \mathrm{dx}$ and for $\mathrm{L}_{\mathrm{S}}$, so that the expression between brackets in Eq. (26) can be calculated from theory and be given as a function $F\left(\mathscr{E}_{0 j}\right)$. Then Eq. $(26)$ reduces to

$$
\delta=\sum_{\varepsilon_{0 j}} G\left(\mathscr{G}_{0 j}\right) P_{0}\left(\xi_{0 j}\right) F\left(\mathcal{G}_{0 j}\right) \Delta \mathcal{G}_{0 j}
$$

Each term of the summation over $\xi_{0 \mathrm{j}}$ can be described as the contribution to $\delta$ by electrons generated initially with an energy spread $\Delta \mathcal{E}_{0 j}$ about $E_{0 \mathrm{j}}$. Consider now dividing Figs. 38 to 41 , giving the energy distribution of emitted secondaries, into segments of width $\Delta \mathcal{E}_{0 j}$ centered at $\mathcal{E}_{0 j}$ minus some average energy loss suffered by the electrons in travelling through the film. This exit energy will be labelled $\mathscr{E}_{j}$. Since Figs. 38 to 41 are normalized to unity area, we have for each term in the summation of Eq. 27 .

$$
P\left(E_{j}\right) \delta=G\left(E_{0 j}\right) P_{0}\left(E_{0 j}\right) F\left(E_{0 j}\right)
$$

Both $P_{0}\left(\mathscr{G}_{0 j}\right)$ and $F\left(\mathscr{G}_{0 j}\right)$ are obtained from the theory. $P\left(\mathscr{G}_{j}\right)$ and $\delta$ are known experimentally. This allows finding $G\left(G_{0 j}\right)$ for all $\mathrm{j}$.

The relationship between $\mathscr{E}_{0 \mathrm{j}}$ and $\mathcal{E}_{\mathrm{j}}$ can be estimated approximately from Figs. 17 to 20 by computing an average energy loss for the escaping electrons. Since the assumption is made that $G\left(\mathscr{E}_{0 j}\right)$ is unique for a given material, its 
values can be obtained from the experimental results which are most convenient to use. A thickness of $\tau=500 \AA$ and $E_{p}=7$ or $8 \mathrm{keV}$ will be taken, corres ponding to the experimental results of Figs. 38 to 41 . For these parameters, $-(\mathrm{dE} / \mathrm{dx})$ is essentially constant (see Figs. 4 and 5), so that if one approximates the energy losses to a form $(a x+b)$, the average energy loss is

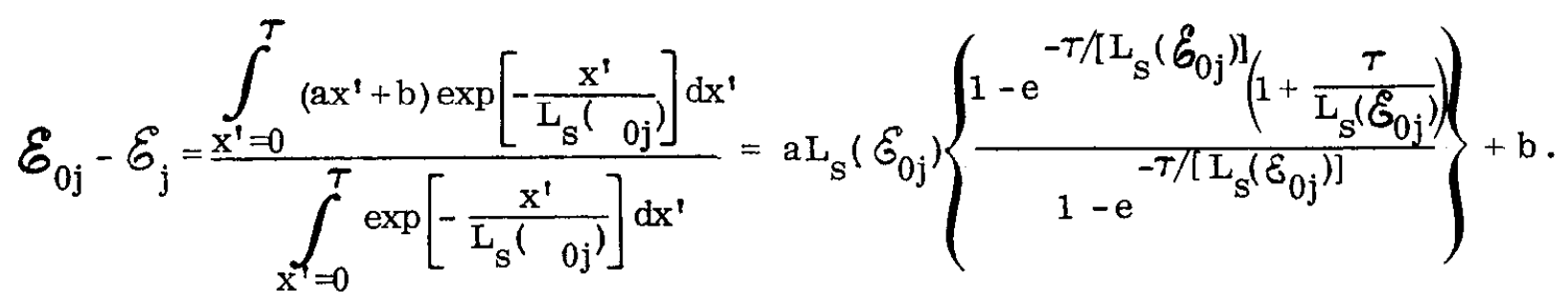

The parameters $\mathrm{a}$ and $\mathrm{b}$ can be obtained approximately from Figs. 17 to 20 . The variable $x^{\prime}$ corresponds to $(\tau-x)$ in previous expressions.

It is realized that this entire procedure is a simple approximation to the problem under study. It can be justified, however, by the lack of structure in the secondary electron energy distributions, with the possible exception of the case of $\mathrm{KCl}$. If the internal generation function $\mathrm{G}\left(\mathscr{E}_{0}\right)$ has structure, its reconstruc tion by the method described above is not expected.

Once the values of $G\left(\mathcal{E}_{0 j}\right)$ have been obtained from consideration of the yield at $\tau=500 \AA, E_{p}=7$ or $8 \mathrm{keV}$, and the exit energy distribution, one should be able to generate curves of $\delta\left(E_{p}\right)$ as a function of film thickness by the use of Eq. (26).

It is interesting to rearrange the summations of Eq. (26) in such a way that a composite probability of escape for all the electrons generated at some location $\mathrm{x}$ can be obtained:

$$
\delta=\sum_{x_{i}}\left(-\frac{d E}{d x}\left(x_{i}\right)\right)\left\{\sum_{\mathscr{E}_{0 j}} G\left(\mathscr{E}_{0 j}\right) P_{0}\left(\mathscr{E}_{0 j}\right) \exp \left[-\frac{\tau-x_{i}}{L_{s}\left(E_{0 j}\right)}\right] \Delta E_{0 j}\right\} \Delta x .
$$

The expression in brackets is the composite escape probability $P(x)$ which has usually been assumed to be a simple exponential. Here, it is a weighted sum of exponentials. 


\section{Calculations for the Selected Alkali Halides}

a. Cesium Iodide

The calculation of $G\left(\mathscr{G}_{0 \mathrm{j}}\right)$ for CsI in accordance with Eq. (28) has been carried out and matched to the yield $\delta$ at $\mathrm{E}_{\mathrm{p}}=8 \mathrm{keV}, \tau=500 \AA$ of Fig. 26 . The average energy losses $\mathcal{E}_{0 \mathrm{j}}-\mathcal{\zeta}_{\mathrm{j}}$ are less than $.1 \mathrm{eV}$ for these materials and have been neglected. From the resulting $G\left(\mathcal{E}_{0 j}\right)$, the value of yield for the six films of different thickness, and for $E_{p}=4$ to $12 \mathrm{keV}$ has been computed. Several computations of $\mathrm{G}$ and the corresponding $\delta$ have been carried out, starting with the values of $\mathrm{L}_{\mathrm{S}}$ given in Table 7 , by increasing or reducing those values by $10 \%, 20 \%$, and $30 \%$. The changes in $\mathrm{L}_{\mathrm{s}}$ affect the computed $\mathrm{G}\left(\mathscr{E}_{0 \mathrm{j}}\right)$ as a match to the yield at $\mathrm{E}_{\mathrm{p}}=8 \mathrm{keV}, \tau=500 \AA$, is prescribed. If the values of $\mathrm{L}_{\mathrm{s}}$ are reduced, the resulting values of $\mathrm{G}$ will increase. With the new values of $\mathrm{G}, \delta$ for small $\tau$ will become higher than previously, while $\delta$ for large $\tau$ will decrease. The best fit to the experimental results of Fig. 26 has been obtained with individual $L_{S}$ values reduced by $20 \%$ from those of Table 7 . The theoretical results are also shown in Fig. 26. For the cases of $\tau=140 \AA$ and $\tau=1000 \AA$, one point has been plotted in each graph showing the fitting obtained with the values of $L_{S}$ from Table 7 , without modification. For $T=1000 \AA$ the point is too high, indicating that $\mathrm{L}_{\mathrm{S}}$ have to be reduced. For $\tau=140 \AA$ the point is too low, even after allowing for the secondary emission from the substrate (maximum yield $\simeq .6$ ). As a test, the values of $\mathrm{L}_{\mathrm{s}}$ for $\mathrm{KCl}$ have been used on CsI, resulting in the points shown in Fig. 26, indicating that the escape depths used are too small for a good fit to the experimental results with CsI.

In the process of computing different fittings, it has been noticed that changes in $\mathrm{L}_{\mathrm{S}}$ of up to $\pm 30 \%$ do not affect the shape of the individual curves much; they simply increase or decrease the magnitude of a given curve with a "pivot" on the $\tau=500 \AA$ curve, which remains untouched. This indicates that discrepancies 
between the shapes of experimental and theoretical curves are due to errors in the calculation of $-(\mathrm{dE} / \mathrm{dx})$ by the procedure described in Chapter I. Since changes in $\mathrm{dE} / \mathrm{dx}$ wre very rapid at the low $\mathrm{E}_{\mathrm{p}}$ end, results there are very sensitive to thickness and actual accelerating voltages of primary electrons in both the present measurements and those of Kanter. ${ }^{6}$ In addition, the fitting of experimental curves by simple functions, the assumptions made regarding the handling of composite films, and the need to compute $\mathrm{dE} / \mathrm{dx}$ for films thicker than the actual value of $\tau$ (in order to determine the constant loss straight lines) can be factors contributing substantial error. The constant loss correction in itself probably results in the observed discrepancies at the high $\mathrm{E}_{\mathrm{p}}$ end. It has been ascertained that leaving out the straight line part of Fig. 4 gives very bad results in the computation of secondary yield. In spite of the above difficulties, the agreement between experimental results and the computed yields is quite good in all the ignificant details. The need to reduce the values of $\mathrm{L}_{\mathrm{S}}$ by $20 \%$ is equivalent to taking the effective mass of the electrons as being $\mathrm{m}^{*} \simeq 1.2 \mathrm{~m}$ for free electrons carrying a cloud of polarization. This interpretation is open to question, however.

The results for $G\left(\hat{G}_{0 j}\right)$ obtained for the theoretical values of $L_{s}$, reduced by $20 \%$, are shown in Fig. 42. The internal generation function is shown to be a monotonically decreasing function of energy. At the low energy end, $G\left(\mathscr{C}_{0 j}\right)$ is shown to have a high value. This result is proportional to the value of $P\left(\mathscr{E}_{j}\right)$ taken from Fig. 38. Because of the spread in energy loss suffered by electrons in their way to the exit surface, there is an uncertainty on which values to give $P\left(\mathscr{G}_{j}\right)$, particularly at the low energy side of a peak in the distribution. The proportionality constant between $G\left(\mathscr{G}_{0 j}\right)$ and $P\left(\mathscr{G}_{j}\right)$ is high at low energies because $L_{s}$ is small [refer to Eqs. (26) and (28)]. Thus, at low energies, substantial error can occur due to an imperfect reading of the exit energy distribution, in comparison to errors incurred at intermediate energies. 


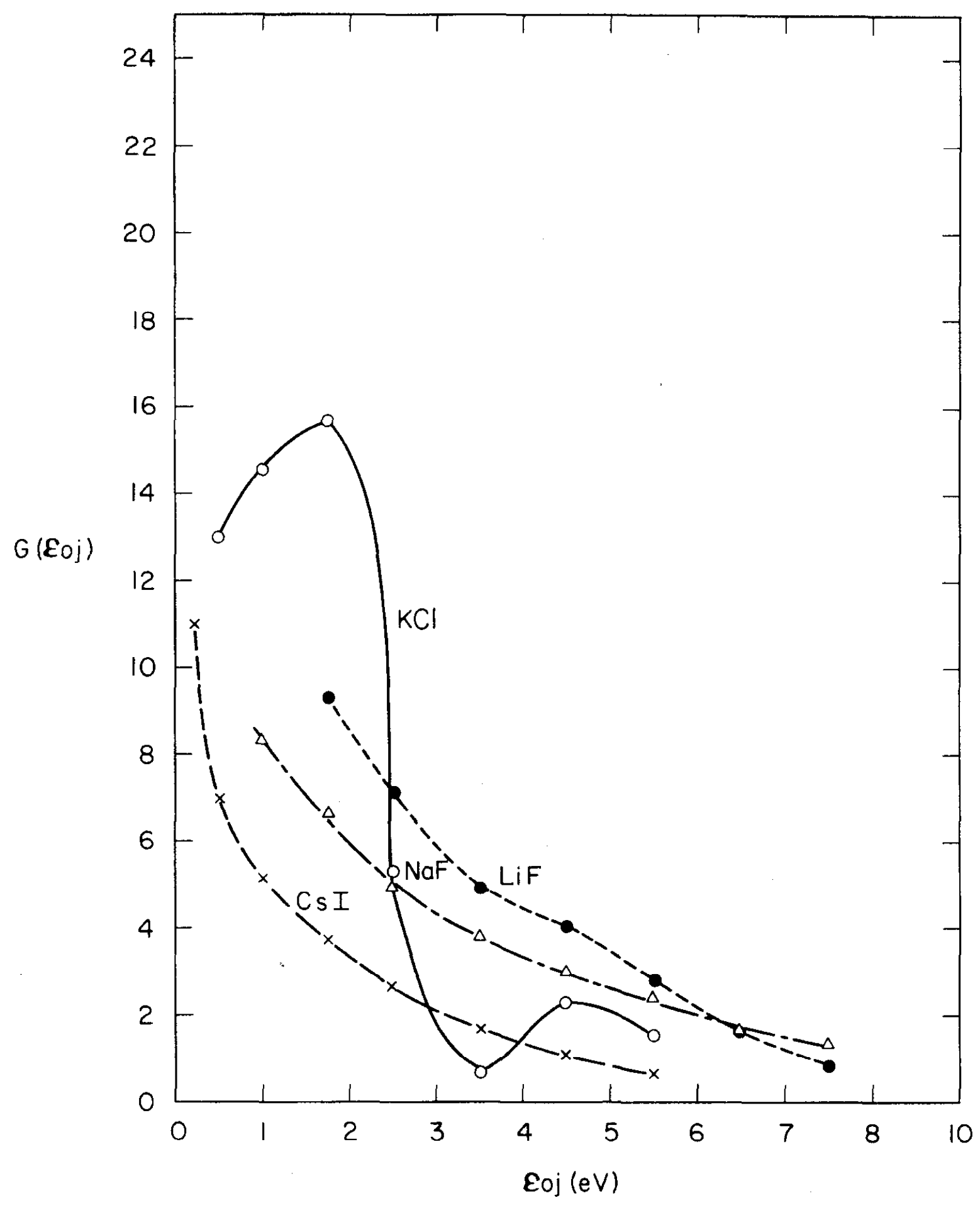

FIG. 42--Internal generation function $G\left(\mathcal{E}_{0}\right)$ for the four selected alkali halides. 
The composite escape depth $\mathrm{P}(\mathrm{x})$ for a $1000 \AA$ film of $\mathrm{CsI}$ is shown in Fig. 43 , as well as for the other three materials studied. For films with $\tau<1000 \AA$, a section of the graph can be taken in such a way that the exit surfaces coincide. The function $\mathrm{P}(\mathrm{x})$ is only moderately close to an exponential. Some local values of $\mathrm{L}_{\mathrm{S}}$ have been shown in the graph. The decrease in local $\mathrm{L}_{\mathrm{S}}$ as one moves towards the exit of the film is due to the increased participation of lower and lower energy secondaries to the secondary yield.

The effect on $\delta, G\left(\hat{0 j}_{j}\right)$ and on $P(x)$ of reducing the value of $P\left(\xi_{j}\right)$ read for very low energy electrons can be easily foreseen: G will decrease at the low energies where the reduction has been effected, while it will increase slightly at other points. This is due to the fitting to one experimental point. The only values of $\delta$ which will be affected substantially will be those for small $\tau$ because low energy electrons, with low $\mathrm{L}_{\mathrm{S}}$, play a relatively more important role in secondary emission in those films. In the escape function $P(x)$, a decrease in $G$ at the low energy end will tend to make the graph change slope (in semilogarithmic paper) more slowly near the exit side of the film.

The aim of this discussion is to show that changes in the behavior of electrons with small $L_{s}$ (either by reduction of $P\left(G_{j}\right)$ or by some actual manipulation on the values of $\mathrm{L}_{\mathrm{S}}$ ) cannot be distinguished very well in a comparison of experimental vs. theoretical yield curves.

\section{b. Potassium Chloride}

The numerical results obtained for $\mathrm{KCl}$ shown in Fig. 27 have turned out in substantially less agreement with experiments than in the case for CsI. Application of the method indicated above for finding $G\left(\xi_{0 j}\right)$, by fitting the computed yield to the single experimental point at $\tau=525 \AA, \mathrm{E}_{\mathrm{p}}=8 \mathrm{keV}$, and using the values of $L_{s}\left(\varepsilon_{0 j}\right)$ of Table 7 , resulted in an internal generation function with very high values of $\mathrm{G}$ at $E_{0}=.5 \mathrm{eV}$ and $1 \mathrm{eV}$. It was felt that perhaps the escape length calculated for those electrons might be too low and the values for $\mathrm{L}_{\mathrm{S}}$ were increased arbitrarily to $80 \AA$ and $180 \AA$, respectively, in order to de-emphasize 


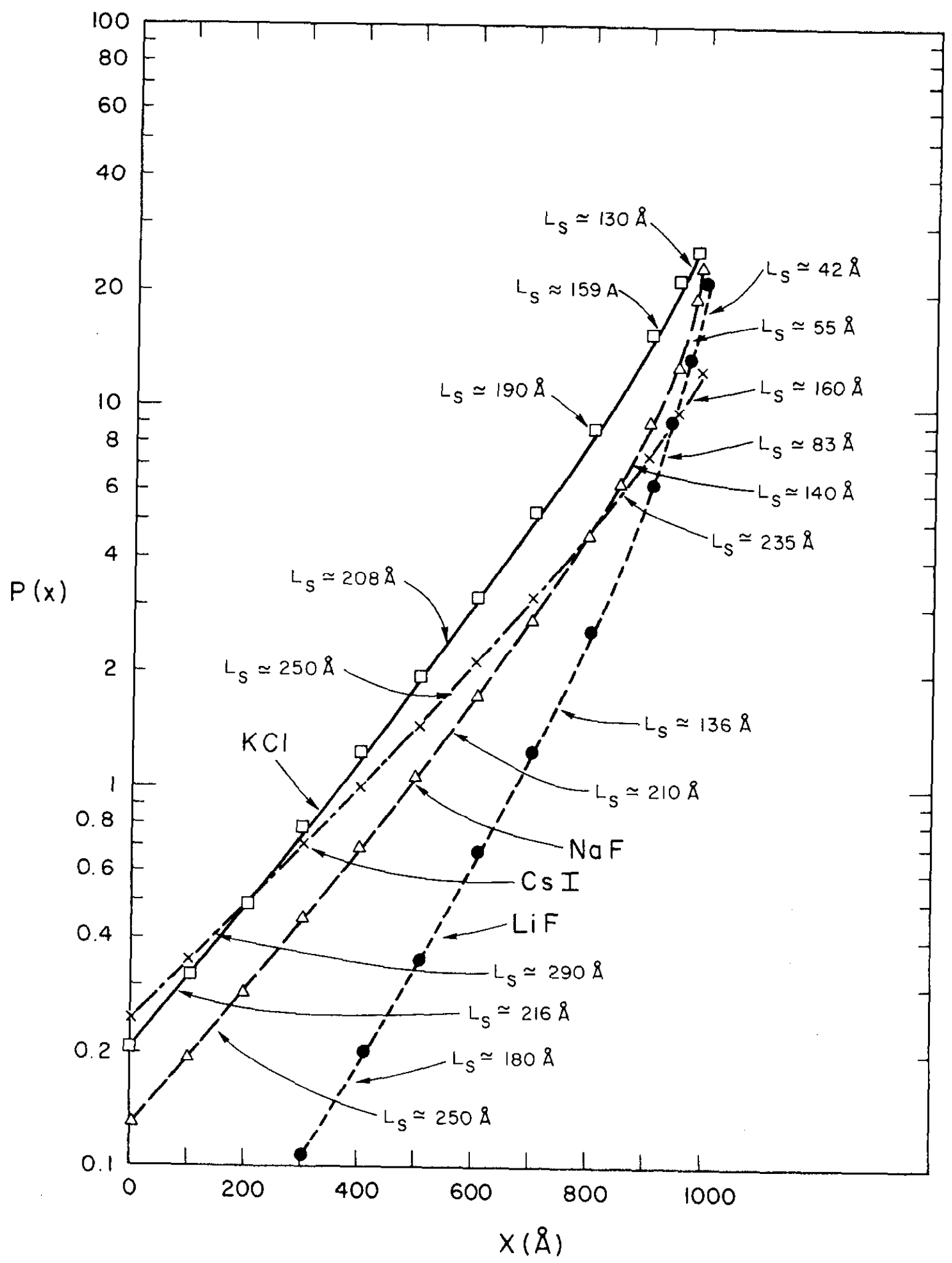

987649

FIG. 43--Composite escape function $P(x)$ for the four selected alkali halides 
their role. Further, all values of $\mathrm{L}_{\mathrm{s}}$ were dacieased by $20 \%$ as the results for $\delta$ indicated that current values for $\mathrm{L}_{\mathrm{s}}$ were too high. In spite of these modifications the results for $\delta$ fail to agree with the experiments in the following points:

1. The shift in the position of maximum yield observed experimentally when $\tau$ is increased does not appear in the computed curves. This is due to the difficulties in the computation of $\mathrm{dE} / \mathrm{dx}$ mentioned previously which become more acute with low density materials ( $\rho=2$, vs. 4 for CsI). In particular, one has to compute the energy losses for a thickness much larger than the one of the film desired in order to find the linear loss corrections, and then take a small number of slabs from results like those of Fig. 5 for the computation of yields.

2. The value of the maximum yield as a function of $\tau$ is shown experimentally to increase to a maximum and then to decrease. In the computed curves, the value of the peak of $\delta$ continues to increase up to $\tau=1000 \AA$. This error is again caused by an unsuccessful calculation of $d E / d x$. In fact, looking at Fig. 6 , it is seen that the curve for $\mathrm{E}_{\mathrm{p}}=6 \mathrm{keV}$ is increasing between $750 \AA$ and $1000 \AA$. On the other hand, the composite escape function $P(x)$ shown on Fig. 43 for $\mathrm{KCl}$, obtained with the modified values of $\mathrm{L}_{\mathrm{s}}$, must be quite good. This can be shown by noticing that for $E_{p}=10$ to $12 \mathrm{keV}$, when $\mathrm{dE} / \mathrm{dx}$ is a straight line for all $\tau$ up to $1000 \AA$, the computed values of $\delta$ reach a "saturation" at thicknesses of $750 \AA$ and $1000 \AA$ and so do the experimental results.

3. The shape of the computed curves at low $\mathrm{E}_{\mathrm{p}}$ does not coincide with the experimental curves as well as in the case of CsI. The reason is the same as in the above paragraph.

4. At small $\tau$ the computed results are not lower than the experimental ones. This is necessary in order to account for secondary emission from the 
substrate. It is felt that this difficulty would be due to either the same reason as above or else to wrong values of $P\left(\mathcal{E}_{j}\right)$ or $L_{s}\left(\mathscr{E}_{0 j}\right)$ for low energy secondaries, as they play a relatively large role in the thinner films. For these reasons, and on account of the somewhat uncommon appearance of the internal generation function shown in Fig. 42 , it is necessary to look at the results for $\mathrm{KCl}$ with some reservation.

One must point out, however, that the results for the escape probabilities seem quite correct, particularly with a decrease of $20 \%$ in the values of $\mathrm{L}_{\mathrm{s}}$. For the very low energy secondaries, the same questioning as to the validity of the perturbation calculations occurs again.

\section{c. Sodium and Lithium Fluorides}

The computed values of $\delta$ for NaF and LiF are shown in Figs. 28 and 29, respectively. The single fitting points between the theoretical and experimental results were $\tau=500 \AA$, $\mathrm{E}_{\mathrm{p}}=7 \mathrm{keV}$ for $\mathrm{NaF}$, and $\tau=500 \AA \mathrm{A}_{\mathrm{p}}=8 \mathrm{keV}$ for $\mathrm{LiF}$. The same type of difficulties appearing on $\mathrm{KCl}$ have also occurred for these two materials but the results for $\mathrm{NaF}$ are a little better due to higher density than $\mathrm{KCl}$ and higher $\mathrm{Z}$ than $\mathrm{LiF}$. This allows for a better computation of $\mathrm{dE} / \mathrm{dx}$. The shifting of the peak of $\delta$ vs. $\mathrm{E}_{\mathrm{p}}$ as $\tau$ is increased appears in the results for $\mathrm{NaF}$, although not to the extent of the experimental results. Also, the "saturation" of the yield as $\tau$ is increased for the higher values of $\mathrm{E}_{\mathrm{p}}$ occurs in agreement with experiments for both materials, indicating that the values of $\mathrm{L}_{\mathrm{S}}\left(\widehat{G}_{0 \mathrm{j}}\right)$ used for the calculations are essentially correct. These have been taken from Table 7 and reduced again by $20 \%$ for a better fit.

The internal generation functions for the two materials are shown in Fig. 42. They have a general appearance similar to that of CsI without the irregularities of the function for $\mathrm{KCl}$. At the low energy end it is not possible to know what G looks like.

The composite escape functions $\mathrm{P}(\mathrm{x})$ are shown in Fig. 43. Local values are shown. As expected, they can only be represented roughly by a single exponential. 


\section{Temperature Dependence}

It was indicated in Chapter I that Dekker ${ }^{35,36}$ determined the ratio of secondary yields for ionic crystals in reflection by a simplified random walk theory, giving as a result

$$
\frac{\delta_{1}}{\delta_{2}}=\left[\left(2 \mathrm{n}_{\nu 2}+1\right) /\left(2 \mathrm{n}_{\nu 1}+1\right)\right]^{1 / 2}
$$

where $\mathrm{n}_{\nu \mathrm{i}}=1 /\left[\exp \left(\hbar \omega / \mathrm{k} \mathrm{T}_{\mathbf{i}}\right)-1\right]$. Stuchinskii ${ }^{37}$ made some.measurements on $\mathrm{MgO}$ and found them to agree quite well with the above result.

It is necessary to investigate whether the above model for secondary emission, when applied to reflection, gives a temperature dependence similar to the one predicted by Eq. (29).

It was discussed at the end of Chapter I that if electrons of a certain energy have an exponential probability of escape given by an escape length $L_{s}$ at room temperature $\left(300^{\circ} \mathrm{K}\right)$, the escape length becomes $\mathrm{L}_{\mathrm{S}} / \beta$ for a temperature $\mathrm{T}$, where

$$
\beta=\left(2 \mathrm{n}_{\mathrm{q}}(\mathrm{T})+1\right) /\left(2 \mathrm{n}_{\mathrm{q}}\left(300^{\circ} \mathrm{K}\right)+1\right)
$$

Except for the square root, expressions (29) and (30) are quite similar, although $\beta$ is not easily related to $\delta_{1} / \delta_{2}$.

For reflection secondary emission, the model under discussion would give for a ratio of yields

$$
\frac{\delta\left(300^{\circ} \mathrm{K}\right)}{\delta(\mathrm{T})}=\frac{\int_{E_{0}} \mathrm{G}\left(E_{0}\right)\left\{\int_{\mathrm{X}} \mathrm{G}(\mathrm{x}) \exp \left[-\mathrm{x} / \mathrm{L}_{\mathrm{S}}\left(\hat{E}_{0}\right)\right] \mathrm{dx}\right\} \mathrm{d} \mathscr{E}_{0}}{\int_{\delta_{0}} \mathrm{G}\left(E_{0}\right)\left\{\int_{\mathrm{x}} \mathrm{G}(\mathrm{x}) \exp -\beta \mathrm{x} / \mathrm{L}_{\mathrm{S}}\left(\tilde{G}_{0}\right) \mathrm{dx}\right\} \mathrm{d} \delta_{0}}
$$

Consider the case with $G(x)$ proportional to $\exp -\mathrm{x} / \mathrm{x}_{0} \cdot$ When $\mathrm{x}_{0}$ is small, it could correspond approximately to a low energy primary electron. For $\mathrm{x}_{0} \gg \mathrm{L}_{\mathrm{S}}$, we would have the case of a high energy primary, with an energy loss 
which is almost a constant within a depth $\mathrm{L}_{\mathrm{s}}$. Carrying out the integrals over $\mathrm{x}$ we obtain

$$
\frac{\delta\left(300^{\circ} \mathrm{K}\right)}{\delta(\mathrm{T})}=\frac{\int_{\hat{E}_{0}} \mathrm{G}\left(\xi_{0}\right) \frac{\mathrm{x}_{0} \mathrm{~L}_{\mathrm{s}}\left(\xi_{0}\right)}{\mathrm{x}_{0}+\mathrm{L}_{\mathrm{s}}\left(\xi_{0}\right)} \mathrm{d} \hat{\xi}_{0}}{\int_{0} \mathrm{G}\left(\xi_{0}\right) \frac{\mathrm{x}_{0} \mathrm{~L}_{\mathrm{s}}\left(\xi_{0}\right)}{\beta \mathrm{x}_{0}+\mathrm{L}_{\mathrm{s}}\left(\xi_{0}\right)} \mathrm{d} \xi_{0}}
$$

If one looks at the extreme cases with $\mathrm{x}_{0}$ much smaller than any significant $\mathrm{L}_{\mathrm{s}}$, and $\mathrm{x}_{0}$ much larger than $\mathrm{L}_{\mathrm{s}}$, one finds that the ratio of yields ranges between $\beta$ and 1 . Since $\beta^{1 / 2}$ also falls between $\beta$ and 1 , one can see that Eq. (29) may give quite good results. This is particularly true for MgO since $\beta$ does not depart much from unity between $100^{\circ} \mathrm{K}$ and $500^{\circ} \mathrm{K}$ us ed by Stuchinskii. ${ }^{37}$ for his measurements. Even so, the experimental results of that author appear better fitted by a dependence $\propto \beta$ rather than $\propto \beta^{1 / 2}$ as can be observed from his Fig. 1, and using $\hbar \omega=1300 \mathrm{k}$ (Boltzmann's constant) for MgO.

A better material for a test of temperature dependence would be $\mathrm{KCl}$, for example, with $\hbar \omega \simeq 300 \mathrm{k}$, with more substantial deviations in $\beta$ from unity.

Also, temperature dependence should be a function of primary energy, as electrons with longer $L_{S}$ increase their contribution to yield as $E_{p}$ is increased.

\section{B. Final Discussion and Conclusion}

The results presented in this report show that the proposed model for secondary emission from alkali halides is a valid one. There is an energy loss suffered by primary electrons which can be computed quite successfully for materials with high $\mathrm{Z}$ and density by a differentiation of the experimental results of Kanter ${ }^{6}$ for scattering of electrons in thin foils. Computations for lighter alkali halides cannot be obtained with such high accuracy from those results.

The energy lost by primaries results in the generation of low energy electrons (internal secondaries) with an energy distribution given approximately by 
the graphs of Fig. 42. The behavior of the internal generation function at the lower energy end cannot be determined by the methods used, since very low energy electrons do not manage to come out from the film if the interpretation of the measured secondary energy distributions in the light of the electron-phonon interaction is correct.

The integral $\int_{\mathcal{E}_{0}} \mathrm{G}\left(\mathcal{E}_{0}\right) \mathrm{d} \mathcal{E}_{0}$ over all the energies between 0 and the energy of the first absorption peak (approximately) would give the number of internal secondary electrons generated per $\mathrm{keV}$ of energy lost by the primary beam. In the absence of sufficient information at the low energy end, one can carry out the integral over the parts of the curves shown in Fig. 42. Then one obtains the number of internal secondary electrons(with energies such that they will contribute to secondary emission) per keV of primary energy lost. From Fig. 42 these numbers are, approximately, 20 electrons/keV for $\mathrm{CsI}, 36$ electrons $/ \mathrm{keV}$ for $\mathrm{KCl}, 26 \mathrm{elec}-$ trons $/ \mathrm{keV}$ for $\mathrm{NaF}$, and 28 electrons/keV for LiF. In terms of energy loss needed to create one internal secondary (still with energy 6 such that they can contribute to yield) these figures are:

CsI: $\quad 50 \mathrm{eV}$ per electron generated

$\mathrm{KCl}: 28 \mathrm{eV}$ per electron generated

NaF: 38 eV per electron generated

LiF: $36 \mathrm{eV}$ per electron generated

It is felt that these numbers can only be taken as an indication of the magnitude of energy loss needed per electron generated above a certain $\mathcal{E}$. From this point of view they are in agreement with the figures of 20 to $35 \mathrm{eV}$ per electron obtained by Sternglass. $^{23}$ If one had a complete generation function, from $\mathcal{G}=0 \mathrm{eV}$, the above numbers would be reduced. In the semiconductors $\mathrm{Si}$ and $\mathrm{Ge}$, it is found that the energy required to generate one electron-hole pair is approximately twice the band gap. If this holds for wider gaps, as in alkali 
halides, the above considerations are still consistent, and a large number of very low energy electrons which do not come out at all must be generated.

It is interesting to compare the results of energy distribution of emitted secondaries (Figs. 38 through 41) and the shape of the internal generation function (Fig. 42) with the conclusions reached by Dekker ${ }^{36}$ from a transport theory based on a simplified electron-phonon interaction in insulators. By assuming an internal generation function

$$
\begin{gathered}
\mathrm{G}(\mathscr{E})=\text { constant for } 0<\mathscr{E} \leq \mathscr{E}_{0} \\
\mathrm{G}(\mathscr{E})=0 \text { for } \mathscr{E}>\mathscr{E}_{0}
\end{gathered}
$$

where $\varepsilon_{0}$ is some fixed energy depending on the material, Dekker finds an external energy distribution peaked broadly at $\mathscr{E}=5 \mathrm{eV}$ by taking $\mathscr{E}_{0}=10 \mathrm{eV}$ and taking an electron affinity of $1 \mathrm{eV}$ for the crystal. For a different assumption for the internal generation function,

$$
G(\varepsilon)=\text { constant } \cdot\left(\varepsilon_{0}-\varepsilon_{0}\right) \text {, }
$$

the external energy distribution shifts to a broad peak near $4 \mathrm{eV}$. Dekker concludes that the external energy distribution is not very sensitive to the form of the internal generation function.

In the light of the work presented in this report Dekker's conclusion appears quite erroneous. Since the escape depth of electrons generated at the higher energies is so much greater than that of electrons with low initial energy, the use of expressions (31) or (32) for $\mathrm{G}(\xi)$ can be expected to lead to very substantial changes in the external energy distribution and in secondary yield.

The electron-phonon interaction is sufficient to account numerically for the escape of internal secondary electrons. By making measurements in transmission with varying thickness of alkali halides, it has been possible to establish the "saturation" of yield with increasing thickness and, in the case of CsI where calculations of energy loss are substantially better than in lighter materials, a very 
complete agreement between calculated and experimental yields is obtained. It has been found that a reduction of the calculated escape length $\mathrm{L}_{\mathrm{S}}$ by about $20 \%$ gives the best results. This may be an indication of the fact that electrons are not totally free, but it could also be caused by some trapping effects. There is substantial uncertainty, however, on the calculated values of $\mathrm{L}_{\mathrm{S}}$ for low energy secondaries. Perturbation theory may not apply, and secondary emission meas urements are not very sensitive to the actual behavior of low energy secondaries.

A composite escape function, corresponding to the simple exponential usually assumed in the literature, can be formed for each material. Local values of $\mathrm{L}_{\mathrm{S}}$, shown in Fig. 43, have the following approximate ranges:

$$
\begin{aligned}
& \text { CsI: } 160 \AA<\mathrm{L}_{\mathrm{s}}<290 \AA \\
& \mathrm{KCl}: 130 \AA<\mathrm{L}_{\mathrm{s}}<216 \AA \\
& \mathrm{NaF}: \quad 55 \AA<\mathrm{L}_{\mathrm{s}}<250 \AA \\
& \mathrm{LiF}: \quad 40 \AA<\mathrm{L}_{\mathrm{s}}<180 \AA
\end{aligned}
$$

These figures can be compared to some published values of $\mathrm{L}_{\mathrm{S}}$ (single exponential assumption) which appear in the literature:

Sternglass and Wachtel ${ }^{76}$ measured a value of $\mathrm{L}_{\mathrm{S}}=(2300 \pm 600) \stackrel{\circ}{\mathrm{A}}$ for $\mathrm{KCl}$. This high value was refuted by Edgecumbe and Garwin ${ }^{61}$ who gave a value of $\mathrm{L}_{\mathrm{S}}=(325 \pm 50) \stackrel{\circ}{\AA}$ for $\mathrm{KCl}$. These same authors found an escape depth $\mathrm{L}_{\mathrm{s}}=$ $(500 \pm 50) \mathrm{A}$ for CsI. These values still appear high in comparison with the ranges of $\mathrm{L}_{\mathrm{S}}$ found in the present work. One possible source of error in the measurement of Edgecumbe and Garwin might be the less sophisticated and less well characterized tube which was used for their experiments.

The secondary emission coefficient of a given alkali halide is, then, determined by four main factors: average atomic numbers, density, frequency of the longitudinal optical modes, and size of the band gap. The average atomic number determines how much energy loss the primary beam will suffer per unit depth in mass units $\left(\mu \mathrm{g} / \mathrm{cm}^{2}\right.$, for example). The frequency of the longitudinal optical modes will determine $\hbar \omega$, the energy exchanged per collision and, along 
with $\epsilon_{\infty}$ and $\epsilon$, it defines collision rates. In general, the higher $\omega$, the more energy losses do electrons suffer on their way to the surface and the lower will be the values of $L_{S}$ in units of length $(\AA)$. The density of the material comes into play by relating length to depth in mass units. For a given $Z$ and $L_{s}$, a more dense material will result in more energy loss within the escape depth $L_{S}$, resulting in higher yield. The band gap has the following effect: If one assumes monotonically decreasing internal generation functions extending up to or near the conduction band energy, materials with larger gap may have a contribution to the yield from electrons with substantially higher initial energies $\varepsilon_{0}$ than in the case of smaller gaps. Since it is found that lower $\mathrm{Z}$ materials have the largest gaps, this effect tends to compensate for the smaller primary energy losses and the shorter values of $\mathrm{L}_{\mathrm{s}}$ for a given $\varepsilon_{0}$. Thus, comparing the results for CsI and $\mathrm{NaF}$, we see that the ratio of $\mathrm{Z}$ is approximately $5: 1$, which affects the energy losses per $\mu \mathrm{g} / \mathrm{cm}^{2}$ by a ratio of roughly $1.5: 1$. Since the densities are in a ratio of $4: 2.8$, the energy loss ratio per $\AA$ is in the ratio of $2: 1$ approximately. For a given $\varepsilon_{0 j}$, the ratio of $L_{s}\left(\xi_{0 j}\right)$ is again about $2: 1$ and yet the maximum yield in transmission for $\mathrm{CsI}$ is 8.2 and for $\mathrm{NaF}$ it is about 5 . The composite escape functions in Fig. 43 for the two materials show that the local values of $L_{s}$ are not very different from each other, with the exception of the region near the exit surface, and this compensates in part for the lower energy losses in NaF. Another factor that seems to help $\mathrm{NaF}$ is the observed primary energy loss needed to generate one electron-hole pair, which is lower in the case of NaF. This point, however, is not well understood at present.

The importance of density in determining reflection secondary yield from the alkali halides was recognized by Gomoyunova and Letunov, ${ }^{60}$ who found a strong correlation between density and yield. Examining Fig. 4 of Ref. 60, one can see several instances, however, in which two materials with practically the same 
density have substantially different yield. An attempt to relate these differences to a difference in $\omega$ has failed, however. The relation holds in some cases and not in others. It is interesting to note that those authors obtain higher maximum yield for $\mathrm{NaF}$ than for $\mathrm{KCl}$, which has about the same yield as $\mathrm{LiF}$, from their data. Since reflection and transmission secondary emission are basically the same phenomenon, one must question some of the results of Ref. 60 in view of the measurements reported here.

In conclusion it can be stated that a substantial amount of information has been gained on the two main processes that make up the phenomenon of secondary emission. A detailed treatment from first principles of the primary energy loss is a very complicated problem at low energies. Probably the best treatment is that of Ref. 74 . Their results for $\mathrm{MgO}$ have been compared to the ones calculated by the method used in the present work for the same material, for very thick films ( $\tau=2000$ and $20,000 \AA)$, and found to agree in the general shape and magnitude of the energy loss function, although they are substantially different in detail. It is felt that the semi-empirical approach used here provides a more solid quantitative basis for use in the next step.

The results of perturbation theory for the electron-phonon interaction, when used in conjunction with the primary energy loss calculations, give a good account of the escape mechanism of low energy electrons, with the possible exception of the case of electrons with very low energies. The results of the Montc Carlo calculations should also be of use in studies of alkali halides by photoemission. 


\section{List of References}

1. L. Austin and H. Starke, Ann. Physik 9, 271 (1902).

2. K. G. McKay, Advances in Electronics 1, 65 (1948).

3. H. Bruining, Physics and Applications of Secondary Electron Emission (McGraw-Hill, New York,1954).

4. R. Kollath, "Secondary Electron Emission of Solids Induced by Electron Bombardment," in Handbuch der Physik (Springer, Berlin, 1956), 21, 232.

5. A. J. Dekker, Solid State Physics 6-, 251(Academic Press, New York, 1958).

6. H. Kanter, Phys. Rev. 121, 461 (1961).

7. H. Kanter, Phys. Rev. 121, 677 (1961).

8. H. Kanter, Phys. Rev. 121, 681 (1961).

9. H. Kanter and E. J. Sternglass, Phys. Rev. 126, 620 (1962).

10. G. W. Goetze, A. H. Boerio and M. Green, J. Appl. Phys. 35, 482 (1964).

11. D. L. Emberson, A. Todkill and W. L. Wilcock, Advances in Electronics and Electron Phys., 16 (1962).

12. E. L. Garwin and J. Edgecumbe, Advances in Electronics and Electron Phys., 22 (1966).

13. H. Jacobs, J. Freeley and F. A. Brand, Phys. Rev. 88, 492 (1952).

14. P. A. Wolff, Phys. Rev. 95, 56 (1954).

15. R. Hilsch, Z. Phys. 77, 427 (1932).

16. A. J. Dekker, Physica 22, 361 (1956).

17. L. Apker and E. Taft, Phys. Rev. 79, 964 (1950); 81, 698 (1951); $\underline{82}, 814$ (1951).

18. M. Knoll, O. Hachenberg and J. Randmer, Z. Phys. 122, 137 (1943).

19. E. M. Baroody, Phys. Rev. 78,780 (1950).

20. A. Van der Ziel, Phys. Rev. 92, 35 (1953).

21. D. E. Wooldridge, Phys. Rev. 56, 562 (1939). 
22. A. J. Dekker and A. van der Ziel, Phys. Rev. 86, 755 (1952).

23. E. J. Sternglass, Westinghouse Research Labs., Scientific Paper 6-94410-2 P9, July 23, 1957.

24. H. Jahrreiss, Ann. Physik (7), 14, 325 (1964).

25. A. Bethe, Handbuch der Physik (Springer, Berlin, 1933), 24, 521 .

26. R. O. Lane and D. I. Zaffarano, Phys. Rev. 94, 960 (1954),

27. J. R. Young, Phys. Rev. 103, 292 (1956).

28. H. Mahr, Phys. Rev. 122, 1464 (1961).

29. Ralston and Wilf, Ed., Mathematical Methods for Digital Computers (Wiley , New York, 1960), pp. 110-120.

30. J. E. Holliday and E. J. Sternglass, J. Appl. Phys. 28, 1189 (1957).

31. M. V. Gomoyunova and N. A. Letunov, Soviet Physics - Solid State 7, 2, $311(1965)$.

32. H. Jahrreiss, Proceedings of the International Symposium on Basic Problems in Thin Film Physics, 1965, Niedermayer and Mayer, Ed. (Vanden Hoeck and Ruprecht, Göttingen, 1966).

33. H. Frölich, Proc. Royal Soc. 16,230 (1937).

34. H. Frölich, Proc. Royal Soc. 172, 94 (1939).

35. A. J. Dekker, Phys. Rev. 94, 1179 (1954).

36. A. J. Dekker, Physica 21, 29 (1954).

37. G. B. Stuchinskii, Soviet Physics - Solid State $\underline{8}, 12,2935$ (1967).

38. W. S. Khokley and K. M. van Vliet, Phys. Rev. 128, 1123 (1962).

39. W. S. Khokley and K. M. van Vliet, J. Appl. Phys. 36, 1714 (1965).

40. C. G. Kuper and G. D. Whitfield, Eds. Polarons and Excitons (Plenum Press, New York, 1963).

41. M. Mikkor, K. Kanazawa, and F. C. Brown, Phys. Rev. 162, 848 (1967)

42. J. A. Hodby, J. A. Borders, F. C. Brown and S. Foner, Phys. Rev. Letters 19, 952 (1967). 
43. Karvel Thornber, Stanford University, private communication.

44. J. M. Ziman, Electrons and Phonons (Oxford University Press, 1960), Ch. 1 and 5 .

45. S. Oyama and T. Miyakawa, J. Phys. Soc. Japan 21, 868 (1966).

46. Y. Onodera, M. Okazaki, and T. Inui, J. Phys. Soc. Japan 21, 816 (1966).

47. J. C. Phillips, Solid State Physics 18,55 (Academic Press, New York, 1966).

48. T. DiStephano, Work in progress, Stanford University, (private communication).

49. W. F. Krolikowski, "Photoemission Studies of the Noble Metals, the Cuprous Halides and Selected Alkali Halides," Stanford Electronics Lab. Tech. Report $5218-1$, May 1967.

50. H. Fröhlich, Advances in Physics 3 , 325 (1954)/

51. H. Fröhlich, Theory of Dielectrics (Oxford University Press, 1958), Sec. 18.

52. Thor A. Bak, Ed., Phonons and Phonon Interactions (Benjamin, New York, 1964), p. 296.

53. G. A. Baraff, Phys. Rev. 135, A528 (1964).

54. J. M. Hammersley and D. C. Handscomb, Monte Carlo Methods (Methuen, London, 1964), Sec. 3.4.

55. J. E. Eby, K. J. Teegarden, and D. B. Dutton, Phys. Rev.116, 1099(1959).

56. K. Teegarden and G. Baldini, Phys. Rev. 155, 896 (1967).

57. E. A. Taft and H. R. Philipp, J. Phys. Chem. Solids 3,1 (1959).

58. E. D. Cashwell and C. J. Everett, Practical Manual on the Monte Carlo Methods in Random Walk Problems (Pergamon, New York, 1959), Ch. 10.

59. W. A. Harrison, private communication.

60. M. V. Gomoyunova and N. A. Letunov, Soviet Physics - Solid State 7, $2,316(1965)$.

61. J. Edgecumbe and E. L. Garwin, J. Appl. Phys. 37,2916 (1966).

62. J. Edgecumbe and E. L. Garwin, J. Appl. Phys. 37, 3321 (1966). 
63. H. Jacobs, Phys. Rev. 84, 877 (1951).

64. A. Roder, "Aluminum Oxide Self-Supporting Films," SLAC Technical Note TN-67-26, Stanford Linear Accelerator Center, Stanford, California (1967).

65. J. R. Young, J. Appl. Phys. 28, 512 (1957).

66. A. H. F. Muggleton and F. A. Howe, Nuc. Instr. and Methods 28,242 (1964).

67. A. B. Gillespie, Signal, Noise and Resolution in Nuclear Counter Amplifiers (McGraw Hill, New York, 1953).

68. E. Fairstein, IRE Trans, in Nuclear Science, NS-8, No. 1, 129 (1961).

69. R. L. Chase, W. A. Higinbotham, and G. L. Miller, IRE Trans. in Nuclear Science, NS-8, No. 1, 147 (1961).

70. V. Radeka, IEEE Trans, in Nuclear Science, NS-11, No. 3, 358 (1964).

71. K. H. Geyer, Ann. Physik (5), 41, 117 (1942).

72. B. Petzel, Ann. Physik (7), $\underline{6}, 55$ (1960).

73. H. J. Boll, "The Study of Charge Trapping Centers in MgO Thin Films," unpublished thesis, University of Minnesota, 1962.

74. D. E. Anderson, A. B. Laponsky, W. T. Peria, et al., "Physics of Electron Emission," University of Minnesota, AFAL-TR-66-250-Vol. 1. (1966).

75. A. A. Schultz and M. A. Pomerantz, Phys. Rev. 130, 2131 (1963).

76. E. J. Sternglass and M. M. Wachtel, Phys. Rev. 99, 646(A) (1955). 